Award Number:

W81XWH-10-1-0787

TITLE:

Regenerative Medicine and Restoration of Joint Function

PRINCIPAL INVESTIGATOR: Rita Kandel

CONTRACTING ORGANIZATION: Samuel Lunenfeld Research Institute Mount Sinai Hospital

Toronto, Ontario CANADA M5G 1X5

REPORT DATE: December 2014

TYPE OF REPORT: Final

PREPARED FOR: U.S. Army Medical Research and Materiel Command Fort Detrick, Maryland 21702-5012

DISTRIBUTION STATEMENT: Approved for Public Release; Distribution Unlimited

The views, opinions and/or findings contained in this report are those of the author(s) and should not be construed as an official Department of the Army position, policy or decision unless so designated by other documentation. 


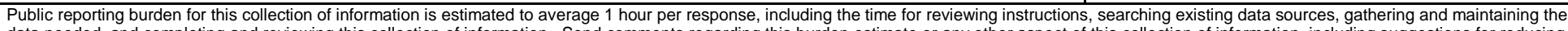

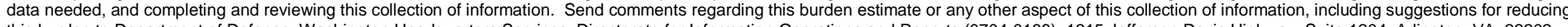

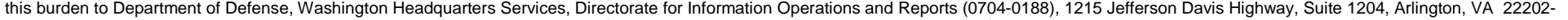

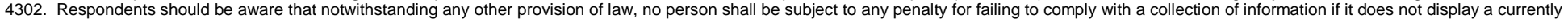
valid OMB control number. PLEASE DO NOT RETURN YOUR FORM TO THE ABOVE ADDRESS.

\begin{tabular}{l|l} 
1. REPORT DATE & 2. REPORT TYPE
\end{tabular}

December 2014 Final

4. TITLE AND SUBTITLE

Regenerative Medicine and Restoration of Joint Function

\section{DATES COVERED}

27Sep2010 - 26Sep2014

5a. CONTRACT NUMBER

W81XWH-10-1-0787

5b. GRANT NUMBER

5c. PROGRAM ELEMENT NUMBER

6. AUTHOR(S)

Dr. Rita Kandel

randel@mtsinai on ca

7. PERFORMING ORGANIZATION NAME(S) AND ADDRESS(ES)

Samuel Lunenfeld Research Institute

Mount Sinai Hospital

Toronto, Ontario CANADA M5G 1X5

9. SPONSORING I MONITORING AGENCY NAME(S) AND ADDRESS(ES)

U.S. Army Medical Research and Materiel Command

Fort Detrick, Maryland 21702-5012

5d. PROJECT NUMBER

5e. TASK NUMBER

5f. WORK UNIT NUMBER

8. PERFORMING ORGANIZATION REPORT NUMBER

10. SPONSOR/MONITOR'S ACRONYM(S)

11. SPONSOR/MONITOR'S REPORT NUMBER(S)

12. DISTRIBUTION / AVAILABILITY STATEMENT

Approved for Public Release; Distribution Unlimited

\section{SUPPLEMENTARY NOTES}

\section{ABSTRACT}

Currently amputation, arthrodesis (joint fusion), or joint replacement are used to treat a joint with an intra-articular fracture or destroyed by a combat injury. Generation of personalized, anatomically shaped biological implants formed using techniques of regenerative medicine in conjunction with biodegradable biomaterial structures to restore a damaged articular joint surface to normal tissue structure, form and function is one way to overcome the limitations associated with current treatment methods. The aims of this study are to: 1) identify the parameters that generate anatomically shaped bone substitutes of optimal composition and structure with an articulating profile. 2) to develop a source of chondrocytes that can generate sufficient amounts of a cartilage layer to cover the bone substitute; and 3) to evaluate the structures formed in a preclinical model. The ongoing studies will further our understanding of the regulation of cell differentiation to chondrocytes and the bone substitute properties required to form a biological joint replacement.

\section{SUBJECT TERMS}

Nothing Listed

16. SECURITY CLASSIFICATION OF:

a. REPORT
17. LIMITATION OF ABSTRACT

UU
18. NUMBER OF PAGES

147 19a. NAME OF RESPONSIBLE PERSON USAMRMC

19b. TELEPHONE NUMBER (include area
code) 


\section{Table of Contents}

Page

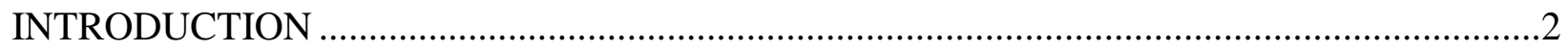

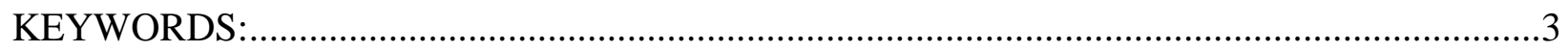

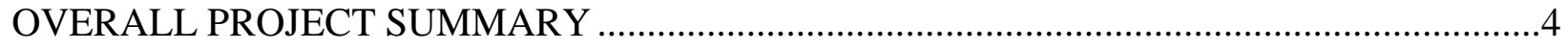

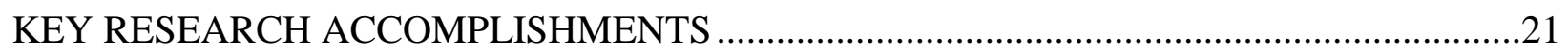

CONCLUSION

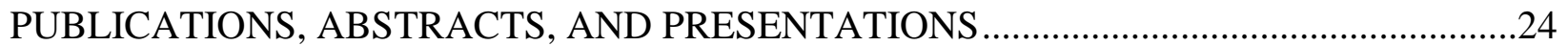

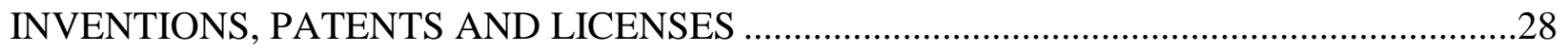

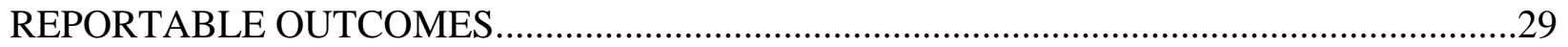

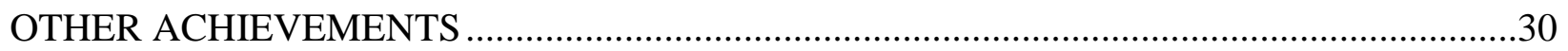

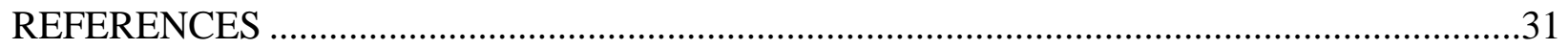

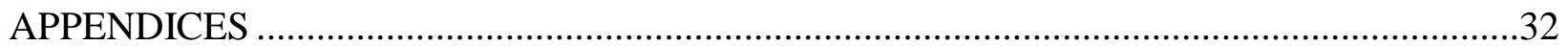




\section{INTRODUCTION}

The complexity of extremity injuries as a consequence of battlefield trauma requires multifaceted reconstructions and has resulted in the need to develop entirely new treatment options to achieve limb salvage and thus full rehabilitation(1-9). The overall aim of this research project was to develop large anatomically shaped biological implants formed using techniques of regenerative medicine in conjunction with biodegradable biomaterial structures to restore a damaged articular joint surface to normal tissue structure, form and function. The surgical methods to evaluate these implants in a pre-clinical sheep model was developed. Our multi-disciplinary team was focused on generating medial tibial plateau and a large segment of the medial femoral condyle (knee joint) biphasic implants (definitive care of battle injuries). The approach we developed has resulted in an implant that can be customized contoured to replace the portion of the knee joint disrupted either by an intra-articular fracture or trauma. Being able to generate personalized implants is a critical feature given that most combat injuries are irregularly shaped.

Using an approach that allows for the formation of living tissues for joint reconstruction offers the advantage of functional tissue integration as well as adaptation to loading conditions during use which should avoid implant failure that can result from the fatigue or wear of synthetic biomaterial. This approach allows the generation of an implant of any contour, making this approach particularly appropriate for individuals who have irregular-shaped defects as a result of a combat injury. Three issues had to be overcome before these large biphasic constructs can be 
used clinically, particularly in the military setting, are 1) the identification of an accessible human cell source to generate a large quantity of cartilage tissue; 2) the methodology to easily, rapidly and reliably generate custom-made CPP bone substitutes of desired shape; 3) develop a pre-clinical animal model to evaluate these implants. This report will summarize the results obtained during this grant period.

\section{KEYWORDS:}

Joint restoration, cartilage tissue engineering, bone substitute biomaterial, cartilage repair 


\section{OVERALL PROJECT SUMMARY}

The following summarizes the work that has been accomplished during the grant period.

\section{Aim 1: To design and make appropriately shaped porous CPP surface replacements for evaluation in a pre-clinical sheep model}

As there did not seem to be an optimal bone interfacing material, we developed a biodegradable inorganic polymer (calcium polyphosphate, CPP) that can be formed as a porous component by sintering CPP particles of selected size under controlled conditions. The CPP powders are formed from calcium and phosphate and when sintered to form porous structures using appropriate processing conditions that we have developed in previous studies, they do not incite an adverse reaction as they slowly degrade in vivo. The degradation rate is dependent on factors such as impurity levels, degree of crystallinity, crystal size and free surface area (related to percent porosity and particle size). The porous CPP structures can be made to have mechanical properties similar to cortical or cancellous bone making them suitable to use as a bone substitute to form joint replacements. The properties (strength and pore size) of the porous CPP can be modulated for the required need through selection of CPP particle size and sintering conditions and can be made sufficient for weight bearing. Although porosity can be as low as $30 \%$, bone ingrowth still occurs likely because of the microporosity. Thus porous CPP is suitable for facilitating cartilage anchorage to bone as well as bone defect filling in a biological joint replacement. However one potential limitation to using porous CPP is the need to generate the substrate easily, rapidly, and reliably in any desired shape in order to be able to repair/regenerate a joint injured during combat 
or training. We accomplished this via additive manufacturing, so-called solid freeform fabrication.

The goal of the studies in aim 1 was to: Design and make appropriately shaped porous CPP surface replacements for evaluation in a pre-clinical sheep model. Thus the focus of our studies was to optimize the solid freeform fabrication (SFF) of large anatomically correct shaped segment of the medial femoral condyle as well as the medial tibial plateau in order to generate biphasic implants for evaluation in a sheep model.

Task 1. Material Optimization for the SFF Process:

We identified appropriate CPP and PVA compositions and particle sizes to facilitate spreadability of the mixture in the newly developed SFF system. In addition, a photopolymer (Ethoxylated-10 bisphenolAdiacrylate) photopolymer solute ion was identified and used for making macro-size conformal channels within the CPP substrates. The sacrificial photopolymer is fully removed from the scaffolds post-processing to prevent any side-effects as a result of adverse biological responses. It was shown that the proposed photopolymer is fully removed by exposure of scaffolds to a predefined heat treatment protocol. Material characterizations conducted via Scanning Electron Microscope (SEM) using an Energy-Dispersive X-ray Spectroscopy (EDS), X-Ray Diffraction (XRD) and Differential Scanning Calorimetry (DSC)Thermo Gravimetric Analysis (TGA) suggested that the photopolymer was fully disintegrated and no secondary residuals were produced during the heat treatment process. A variety of structures have been made to assess the role of macro- size conformal channels on mechanical strengths. An in-vivo rabbit study was conducted and the results showed bone ingrowth with all 
samples resulting in 30-40\% fill of available porosity by bone within the 6-wk period. In the 6wk in vivo period, approximately 7-9\% loss of CPP by degradation had occurred.

\section{Task 2. Optimization of SFF Rolling Compaction Parameters:}

The SFF rolling compaction parameters to control the density of green samples was optimized. Based on our results, we concluded that the roller spinning and linear speeds do not change the green density considerably, whereas the stacking layer thickness may effectively control the green density. It was also noted that the roller speeds would only be changed in a specific range where speeds out of this range may lead to crack formation and non-uniformity in the spread powder within the build compartment of SFF system. The results indicated that an increase in stacking layer thickness from 150 to 250 um increases the green density from $39 \%^{+-2}$ to $46 \%^{+-}$ 2. This increases controllability of the final porosity of CPP implants with direct effect on the mechanical strength of the biomaterial. The lower green density, the higher porosity and the lower mechanical compression strength. This approach allowed us to make functionally graded implants with varying porosity where we could change the stacking layer thickness during the fabrication to arrive at heterogeneous porosity across the implant if desired.

Task 3. Fabrication of the Large Anatomically Correct Segmental Replacement with Multiple CPP Powder Sizes:

We imaged sheep knees by CT and after multiple iterations developed anatomically shaped medial tibial plateau and femoral condyle parts that are suitable to use for the implantation studies (Figure 1). These shapes are made by SFF. To this end, a an additive manufacturing 
design cycle was developed, which included an algorithm for computing anisotropic shrinkage and appropriate compensation factors along the $\mathrm{x}, \mathrm{y}, \mathrm{z}$ printing directions to ensure that the resulting complex shape retained shape fidelity after thermal annealing when compared to an ideal benchmark. This process was iterative in nature. The algorithm was tested on a medial tibial plateau and femoral trochlea bone substitute for a sheep model and can be expanded to other applications. In the first iteration, the compensation factors were determined to be approximately $+30,+20$, and $+10 \%$ in the $x, y$, and $z$ directions respectively. Within the second iteration, the part fidelity was brought to within $-6,+6$, and $+7 \%$ of the final part dimension in the $\mathrm{x}, \mathrm{y}$, and $\mathrm{z}$ directions respectively. In addition, the use of two powder sizes, small powder $<75 \mu \mathrm{m}$ and large powder 75-150 $\mu \mathrm{m}$, influenced structural and mechanical properties of manufactured parts. It was shown that the powder size resulted in a range of bulk density of $1.27-1.46 \mathrm{~g} / \mathrm{cm} 3$, porosity of 49-55\%, and compression strength of 2.9-15.5 MPa. Experimental results indicated that the capability of selecting different powder sizes can have an effect over controlling the functionally graded structural and mechanical properties of the manufactured construct.

\section{Task 4 Fabrication of the Targeted Model with Oriented Porosity}

We determined the role of oriented porosity on the mechanical strength of substrates. The mechanical properties of the $35 \%$ porous structures were characterized by uniaxial compression testing for compressive strength determination and diametral compression testing to determine tensile strength. Fracture cleavage surfaces were analyzed using scanning electron microscopy. The effects of the fabrication process on the microarchitecture of the CPP samples were also investigated. Results suggest that the orientation of the stacked layers has a substantial influence 
on the mechanical behavior of the SFF-made CPP samples. The observed anisotropic mechanical properties were analyzed based on the physical microstructural properties of the CPP structures.

In summary, experimental data showed that, the bulk density varied between $1.8-2.0 \mathrm{~g} / \mathrm{cm}^{3}$, the bulk porosity ranged between 30-38\%, while the compressive strength varied between 13.4-45.1 MPa. In this work, it was shown that control over functionally graded properties can be achieved by selecting the print layer orientation within the part.

\section{Aim 2: Optimize the conditions to form cartilage on larger surfaces using human chondrocytes}

One major problem limiting the clinical application of bioengineered cartilage for joint repair is identifying a source of sufficient numbers of differentiated chondrocytes to form enough articular cartilage to cover a large surface replacement(10). Chondrocytes de-differentiate when passaged (to expand cell number) even once in monolayer culture. A variety of approaches have been developed to circumvent this but none entirely successful. To translate this biphasic implant approach into clinical practice we must be able to generate large amounts of cartilage from human chondrocytes, which we can do reliably using a co-culture approach. However this requires, at present, the use of bovine chondrocytes. The following experiments are designed to determine the condition that favours cartilage formation suitable to use for joint resurfacing.

Task 5: To generate chondrocytes from iPS cells that can serve as a source of chondrocytes to form the cartilage layer of the large biphasic implants 
5a. Generate iPS cells from chondrocytes and characterize phenotype: We developed the methodology to transfect cells with the genes c-Myc, Klf4, Oct4 and Sox2 (single piggyBac (PB) cassette whose expression is under doxycycline regulation and will also provide neomycin resistance). The transfected cells were selected for by monolayer culture in the presence of G418 until 100\% of the untransfected (control) cells have died. The G418 is then removed and the transfected cells allowed to proliferate. We evaluated a number of experimental conditions to generate these cells. We tried different ways to transfect chondrocytes including fugene, lipofectamine, and Neon with transfection rates of less than 10\%. A number of pretreatments (e.g. enzymatic) were attempted as well as varying cell density during transfection. Chondrocytes after different numbers of passage or even freshly isolated from cartilage have been tried as have both bovine, sheep and human chondrocytes. To date we have not been able to generate colonies, indicative of transdifferentiation to embryonic cells (iPS). Under one condition, transfection of constitutively active genes, have we been able to generate a colony but this is not useful for use clinically as they will not be amenable to differentiation back to chondrocytes.

As an alternate approach, iPS cells were derived from human fibroblasts. Within the last year, we have generated induced pluripotent stem cells (iPSCs) from fibroblasts. iPSCs were generated by retroviral infection of Oct4, Sox2, Klf4, and c-Myc in normal fibroblasts. Over 20 clones were generated. Two iPSC clones were fully characterized. These cells maintain normal karyotype after reprogramming. They express the pluripotency markers SSEA-4 and TRA-1-60. Using an in vitro embryoid body (EB) outgrowth differentiation assay, all iPSC lines were shown 
to differentiate to all three germ layers because they expressed smooth muscle actin (mesoderm marker), GATA4 (endoderm marker), and $\beta$ III-tubulin (ectoderm marker).

To induce chondrogenesis, iPSCs were differentiated using a two-stage process: 1) differentiation towards mesendoderm followed by 2) chondroprogenitor differentiation via micromass culture. iPSCs were grown for 48 hours in E8 (iPSC maintenance media), E6 (iPSC maintenance media without pluripotency growth factors, FGF2 and TGF $\beta 1$ ), or E6 supplemented with BMP4 and Activin A (growth factors known to induce mesendoderm fate). iPSCs grown in the different conditions were harvested and plated at high density (300,000 cells in a 15uL droplet onto a matrigel-coated 12-well plate) as micromass cultures for 7 days. iPSCs initially differentiated in E6 supplemented with BMP4 and Activin A (BA conditions) and sequentially differentiated in micromass cultures expressed the highest levels of sulfated glycosaminoglycans (s-GAG) and hydroxyproline (OH-Pro), components of cartilage tissue (Figure 1A and B). Thus, initial differentiation towards mesendoderm with BMP4 and Activin A aided in the differentiation towards chondroprogenitors from iPSCs.

Task 6: To develop use of human chondrocytes for cartilage tissue formation by identifying putative differentiation factor(s)

6a. Determine if young human chondrocytes induce redifferentiation of passaged human chondrocytes:

Passaging of chondrocytes in culture results in dedifferentiation of the cells and loss of their ability to form hyaline (articular) cartilage tissue. Co-culturing human passaged chondrocytes 
with small numbers of primary bovine chondrocytes (bP0hP2) induced redifferentiation of human chondrocytes. Primary adult human chondrocytes (AhP0), either alone or in co-culture (to induce redifferentiation of passaged cells), did not result in cartilage tissue formation. As bovine articular chondrocytes are harvested from skeletally immature animals, we examined if the inability of adult human chondrocytes to form cartilage tissue is dependent on age or species differences between human and cows. Primary and passaged human fetal chondrocytes were cultured alone and in co-culture. Primary human fetal chondrocytes formed cartilage tissue whereas passaged human fetal chondrocytes did not form cartilage tissue rich in proteoglycans when compared to primary bovine chondrocytes as evidenced by toluidine blue staining and biochemical analysis. Additionally, human fetal primary chondrocytes when combined in coculture with bovine (fhP0bP2) or fetal human passaged cells (fhP0fhP2) were not able to form hyaline cartilage tissue as determined histologically and biochemically. However, bovine chondrocytes induced passaged human chondrocytes to form cartilage tissue. Of note, levels of proteoglycans in tissues formed by culture expanded chondrocytes grown in co-culture with fetal primary chondrocytes were significantly greater than when fetal primary cells were cultured alone. This indicates that primary human fetal chondrocytes do have some capacity to induce redifferentiation, but not to the extent of primary bovine chondrocytes. The data suggests that human cells do not have the same capacity as bovine chondrocytes to induce redifferentiation.

6b. Identify factor(s) present in conditioned media of co-cultures using mass spectroscopy:

Cartilage tissue formed in the presence of fetal bovine serum can not be easily used in humans because of the incorporation of foreign (xenogeneic) antigens. Our studies using bovine 
chondrocytes have demonstrated that it is possible to generate cartilage tissue under serum-free conditions and in the absence of co-culture. We characterized chondrogenic gene changes in the differentiating cells to identify the optimal time to evaluate the conditioned media by mass spectroscopy. We observed that gene expression levels of Sox9 ( a transcription factor involved in chondrogenesis and expressed by chondrocytes) peaked after the first week of culture, similarly levels of aggrecan, and type II collagen increased throughout the first week, while levels of type I collagen decreased significantly after the first week. By 8 days of culture, sox 9 levels were similar to differentiated chondrocytes and the levels of type I collagen had decreased significantly and type II collagen has increased significantly when compared to expression levels at day 0 (dedifferentiated chondrocytes). This suggested that our analyses should be performed on conditioned media from the first week of culture.

Bovine primary chondrocytes (P0) were cultured at low cell density in monolayer culture and passaged twice (P2). Culture of the P2 cells at high density on 3-dimensional type II collagen coated membrane inserts in serum-free media supplemented with insulin, high glucose and dexamethasone resulted in redifferentiation of the passaged cells which regain the ability to form hyaline-like cartilage. P0 cells cultured under the same conditions were not able to accumulate matrix. The matrix molecules released (secretome (conditioned media)) by the P2 cells under matrix accumulating and P0 cells under non-matrix accumulating conditions in the first week of culture were examined by mass spectrometry to determine which molecules may be involved in tissue formation. The samples were alkylated with iodoacetamide and desalted using NAP5 columns. The samples were trypsin-digested and the resulting peptides separated by cation-exchange liquid chromatography. The peptides were subjected to mass spectrometry identification with LC- 
MS/MS, on the ThermoFisher OrbiTrap XL Mass Spectrometer. The raw spectra were analyzed using Mascot and X! Tandem software. Collagen types I, III, and XII, and versican were found at higher levels within the P2 secretome (tissue forming), while type II collagen and COMP were found at higher levels in the P0 secretome (non-tissue forming). There was increased collagen synthesis and retention by P2 cells compared to P0 cells as early as the first 3 days of culture and this was maintained up to 10 days, while proteoglycan synthesis was elevated in P0 cells initially in the first 3 days of culture this decreased and was lower than the amount of proteoglycan synthesized by P2 cells between 8-10 days. Based on these results, immunostaining of selected ECM molecules was performed. Confocal microscopy showed that type XII and II collagens, versican, and decorin were present in the ECM of P2 cells (tissue forming) by one day. In contrast, collagen types I and III and decorin were present in the ECM of P0 cells (non-tissue forming) and type II collagen was present intracellularly. This study suggests that versican and collagens XII and II are the early matrix molecules accumulated and may be necessary to provide the right microenvironment for passaged chondrocytes to form cartilage tissue in vitro.

Our studies suggested that insulin, high glucose, and dexamethasone were critical factors in regulating cartilage tissue generation. Articular chondrocytes were passaged twice to allow for cell number expansion (P2) and cultured at high density on 3D collagen type II-coated membranes in high glucose DMEM media supplemented with insulin and dexamethasone (SF3D). The cells were characterized after monolayer expansion and following 3D culture. The P2 cells showed a progenitor-like antigen profile of 99\% CD44+ and 40\% CD105+ and a gene expression profile suggestive of interzone cells. P2 in SF3D expressed chondrogenic genes and accumulated extracellular matrix (ECM). Down-regulating insulin receptor (IR) with HNMPA- 
(AM3) or the PI-3/AKT kinase pathway (activated by insulin treatment) with wortmannin inhibited collagen synthesis. HNMPA-(AM3) reduced expression of Col2a1, Col10a1, and IR genes as well as Sox 6 and 9. Co-IP and ChIP analysis of HNMPA-(AM3) treated cells showed binding of the co-activators Sox6 and Med12 with Sox9 but reduced Sox9-Col2a1 binding. This suggests that insulin mediated Sox9-Col2a1 binding which contributed to the cartilage tissue formation.

Task 6 (i) To develop mesenchymal stromal cells for cartilage tissue formation with a zone of calcified cartilage (revised SOW)

6(i)a. Determine if sheep mesenchymal stromal cells can be used to form hyaline cartilage with $\underline{\text { a zone of calcified cartilage to strengthen integration of in vitro-formed cartilage to CPP bone }}$ substitute substrate.

Healthy cartilage tissue interfaces with bone in vivo through a zone of calcified cartilage, which enhances mechanical integration by redistributing forces acting at the interface(11). Recapitulation of this architectural feature is critical as a calcified zone will serve to resist the constant shear force from the movement of the joint and prevent the delamination of tissueengineered cartilage $(12,13)$. Bone marrow mesenchymal stromal cells (BMSC) are suitable for creating calcified cartilage, as they can be differentiated to produce cartilage tissues that mineralize their matrix. While chondrocytes from the deep zone of articular cartilage can also yield calcified cartilage in vitro, BMSC can be obtained without donor site morbidity and expanded to a large number. At the same time, in vivo studies have shown that BMSC-derived cartilage can maintain their non-calcified phenotype after being implanted in a joint cavity. Thus 
using BMSCs as a single source of cells to create cartilage tissues with a calcified cartilaginous interface, limiting mineralization of BMSC-derived cartilage to the interface is a significant contribution towards clinical translation of cartilage tissue engineering.

To generate the triphasic implant, BMSCs were predifferentiated to chondrocytes on membrane culture, harvested and then grown on a porous calcium polyphosphate bone substitute substrate in the presence of triiodothyronine (T3). T3 was withdrawn, and additional predifferentiated chondrocytes were placed on top of the construct and grown for 21 days in the presence of beta glycerophosphate. This protocol yielded two distinct zones: hyaline cartilage that accumulated proteoglycans and collagen type II, and calcified cartilage adjacent to the substrate that additionally accumulated mineral and collagen type X. Constructs with the calcified interface had comparable thickness $(600 \pm 50 \mathrm{um})$ and equilibrium modulus $(1 \pm 0.05 \mathrm{MPa})$ similar to native sheep osteochondral tissue. This tissue had a 4 fold higher interfacial shear strength compared to biphasic control constructs (no calcified layer), this property should be sufficient to prevent delamination during weight bearing as suggested by sheep gait studies(14, 15). Importantly the non-calcified portion of the cartilage did not have hypertrophic features (no expression of collagen type $\mathrm{X}$ nor alkaline phosphatase activity) and thus did not mineralize even though $\beta$ glycerophosphate was present in the media.

Using BMSC as the cell source, we engineered an osteochondral-like construct that incorporates a zone of calcified cartilage at the cartilage-bone substitute interface without the use of a scaffold. A stepwise culturing strategy enabled the selective activation of BMSC-derived chondrocytes to induce mineralization only at the interface, while avoiding mineralization in the contiguous 
cartilage tissue, thus conferring localized calcified and hyaline zones to the engineered cartilage tissue. Additionally, this approach is clinically relevant as the BMSC were expanded in media supplemented with autologous serum and the construct cultured in serum-free, defined media (no xenogeneic material was used at any point during culture), so this strategy can be directly translated to in vivo preclinical studies. Manuscript submitted to Osteoarthritis and Cartilage in January 2015.

\section{Aim 3: Pre-clinical evaluation of the joint replacement in vivo}

The goal of these experiments was to evaluate whether a partial hemi-joint replacement large, anatomically correct biphasic segmental replacement of the femoral condyle generated in vitro using SFF can be used to repair large joint surfaces in a pre-clinical (sheep) medial tibial plateau model. As the bone substitute can be shaped to model any part of a joint surface, the parts utilized in these studies will serve as proof-of-concept of the effectiveness of our approach and will be the first step towards the ultimate goal of implanting a total large joint replacement. Furthermore, as it is a large animal clinically relevant sized implants are being evaluated.

Task 7: Pre-clinical evaluation of the joint replacement in vivo:

We developed the techniques to image sheep knees by CT and have developed shaped medial tibial plateau and femoral condyle parts as described in Aim 1 that are suitable to use for the implantation studies. 
We received the necessary approvals to do the pre-clinical study. We developed the surgical technique to implant the part into the sheep knee. We started with the femoral condyle implant. We encountered problems placing the implants, given the size, congruent with the joint surface. To overcome this issue we developed addictive manufacturing methodology to design personalized cutting surgical guides made from acrylonitrile butadiene styrene (ABS) by fused deposition modeling, which is an extruded-based AM technology, specifically for each animal joint based on the CT image and computer aided design (CAD). These guides allowed bone cuts to be made that resulted in a bone bed precisely located and shaped to accept the implant. The next issue we addressed was fixation of the implant without cracking the bone substitute. We redesigned and changed the orientation of the screw holes. This change allowed the anatomically shaped components $(4 \mathrm{~cm} \times 1.5 \mathrm{~cm} \times 1 \mathrm{~cm}$ deep) to be placed into a sheep knee and fixed using titanium screws (Figure 2). The substrate was able to withstand weight bearing in vivo as it was intact at the two month time point. Bone and vascular ingrowth were observed focally in the CPP substrate and the implant was biocompatible as no reaction was seen. However we also encountered problems with ligament laxity after implantation which necessitated modification of the surgical procedure.

We then developed a culture system that allows for formation of the triphasic cartilage constructs evenly over the anatomically shaped surfaces.

We have now solved all the issues related to the pre-clinical studies. However these studies depleted our funds. As we are now in a position to evaluate large multiphasic components in a 
Kandel, R. et al

preclinical model we have applied to PRORP expansion Fund competition for funds to perform the animal studies. 


\section{Supporting Data}

\section{Figure 1}
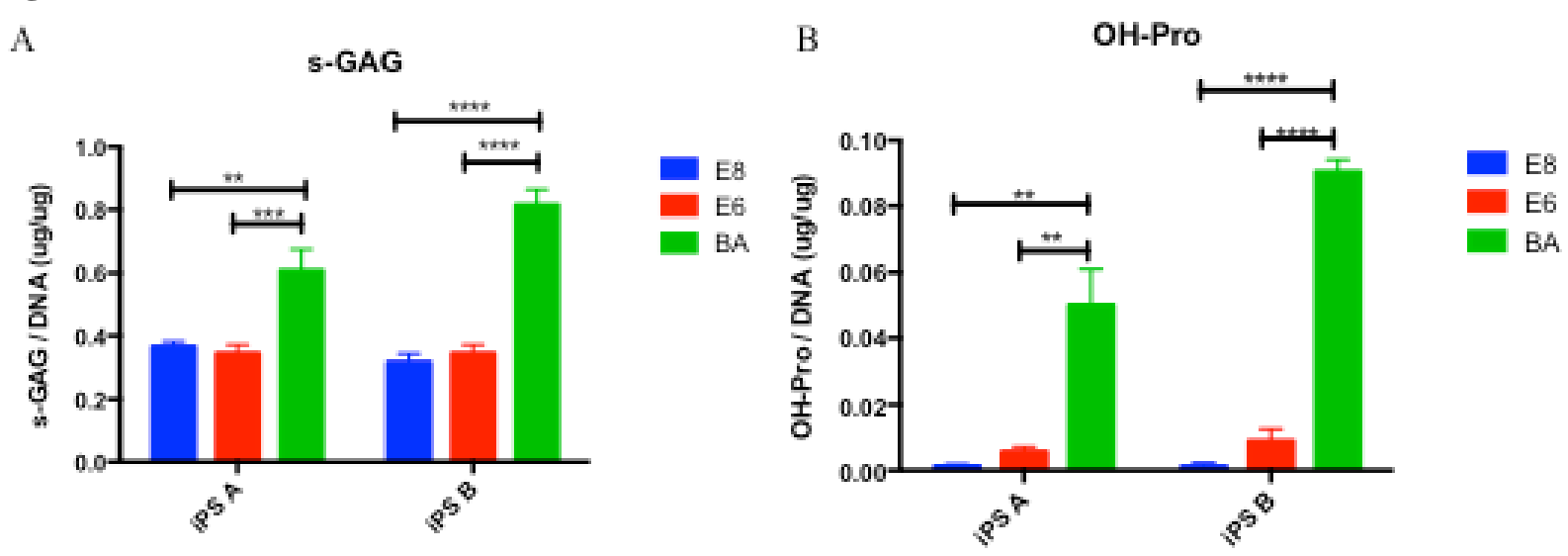

Differentiation of iPSC towards chondroprogenitors. iPSCs were pre-differentiated in E8, E6, or BA (E6 supplemented with BMP4 and Activin A) for 48 hours, harvested, and then differentiated further in micromass culture. Sulfated glycoasminoglycans (s-GAG, A) and hydroxyproline (OH-Pro, B) levels were analyzed after seven days in micromass culture and normalized to DNA content. Oneway ANOVA multiple comparisons test was performed for statiustical analyses. ** $\mathrm{p} \leq 0.01$; **** $\mathrm{p} \leq 0.0001$

\section{Figure 2A}

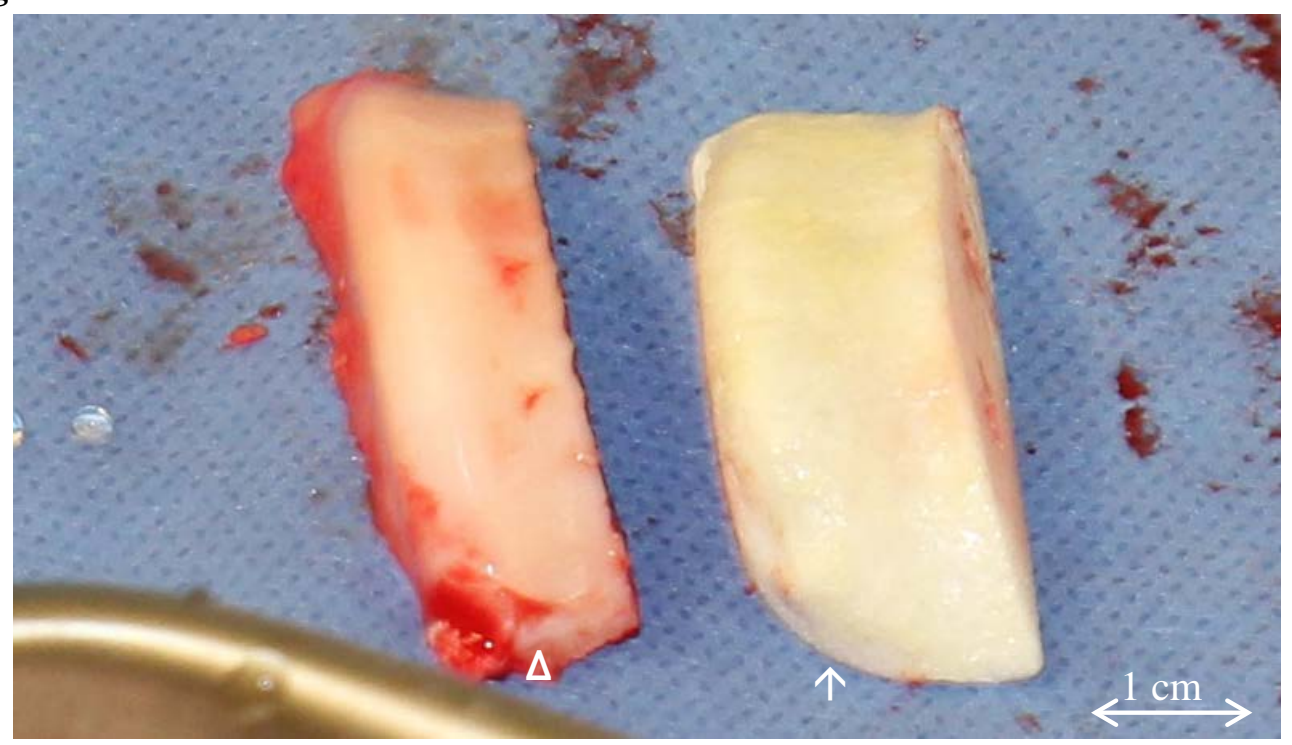

Exercised portion of sheep femoral condyle $(\Delta)$ and the replacement implant $(\uparrow)$ generated by additive manufacturing (SFF). The match is perfect. The difference in width between the two will account for the bone that was removed by the saw during harvest 
Figure 2B

\section{GENERATING A REPLACEMENT PART}
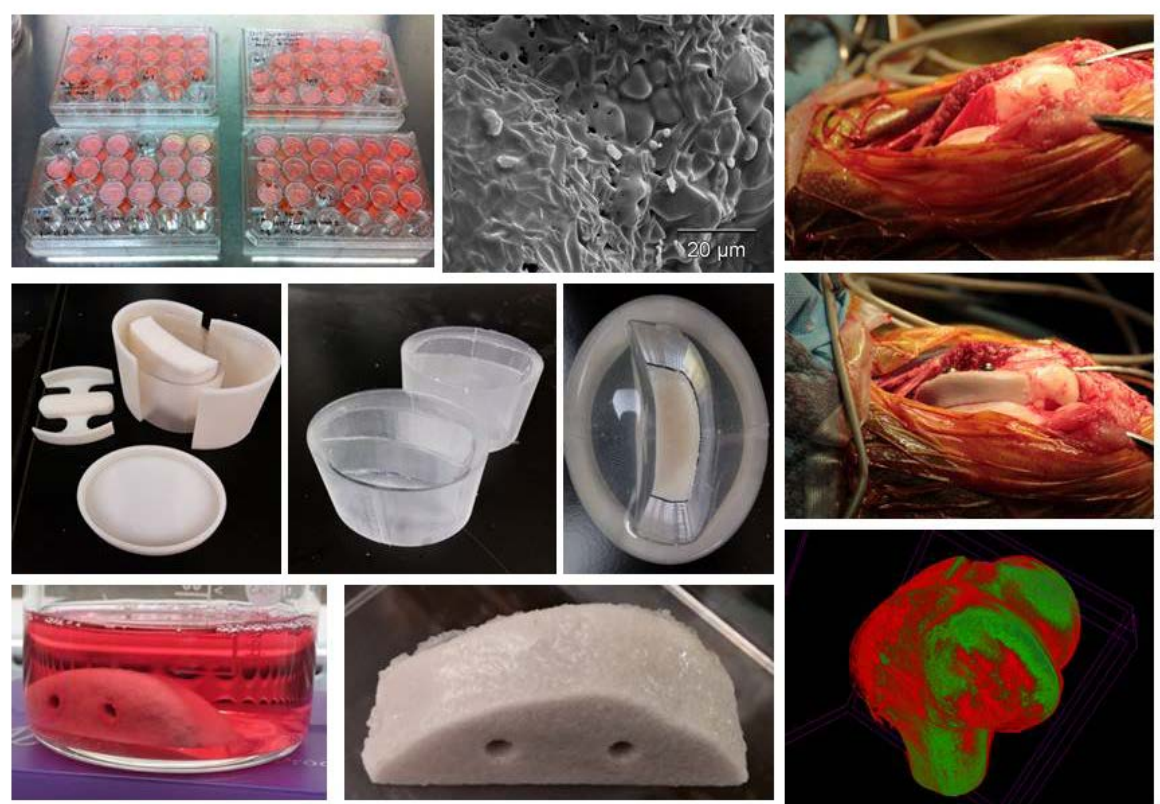

Images showing formation and implantation of anatomically correct portion of sheep femoral replacement part and UCT imaging of implant. 


\section{KEY RESEARCH ACCOMPLISHMENTS}

1) identified appropriate CPP and PVA compositions, CPP particle sizes, and photopolymer to facilitate solid freeform fabrication system (additive manufacturing) to generate parts fabricated from CPP

2) demonstrated that orientation of the stacked layers and the layer thickness appear to have a substantial influence on the mechanical behavior of the solid freeform fabrication made CPP samples

3) generated calcium polyphosphate constructs with oriented porosity

4) animal studies evaluating calcium polyphosphate bone substitutes with oriented porosity formed by solid freeform fabrication in vivo is completed

5) determined that chondrocytes do not easily transdifferentiate into iPS cells and that fibroblasts are a better source of cells.

6) can induce fibroblast generated iPS cells to undergo chondrogenesis.

7) determined that it is not the age of the human chondrocytes that influence the ability of cells to induce redifferentiation of passaged human cells.

8) serum-free culture conditions have been developed to induce chondrocytes to form cartilage tissue and the tissue characterized

9) analysis of secretome and the extracellular matrix accumulated by chondrocytes forming cartilage identified versican and collagens XII and II as the early matrix molecules present in 
the cartilage tissue formed by redifferentiating chondrocytes. Identifying conditions that promote this type of matrix accumulation in human chondrocytes may be important for defining the conditions that favour hyaline cartilage formation for use in regenerative medicine approaches.

10) have identified insulin as the an important factor promoting cartilage tissue formation by redifferentiating cells by inducing sox 9 binding to collagen type II promoter to increase its expression. Collagen type II is present in the early matrix accumulated by these cells.

11) using bone marrow derived mesenchymal stromal cells as a single cell source, an osteochondral-like construct comprised of hyaline cartilage and a porous bone substitute was created with a zone of calcified cartilage interfacing them. As no xenogeneic material was used at any point during culture, this triphasic strategy can be directly translated into preclinical studies

12) design and formation of a large anatomically correct segmental replacement part by additive manufacturing methodology for medial tibial plateau and femoral condyle

13) development and optimization of surgical approach to implant large anatomically correct segmental replacement in femur and tibia

14) development of personalized surgical guides to facilitate surgical implantation of surface replacement

15) development of methodology to grow cartilage on large anatomically correct segmental replacement part 


\section{CONCLUSION}

We are able to design and form large anatomically correct segmental joint replacement parts by additive manufacturing for medial tibial plateau and femoral condyle. This part, which is porous with sufficient strength to withstand weight bearing, supports cartilage growth.

We investigated a number of cell sources including articular chondrocytes passaged to expand cell number, induced pluripotent cells (iPSC), and bone marrow derived mesenchymal stromal cells (BMSC) and found that only BMSC were able to generate the type of cartilage needed, stratified hyaline cartilage with a calcified layer that more closely mimics native cartilage architecture. 


\section{PUBLICATIONS, ABSTRACTS, AND PRESENTATIONS}

\section{Peer Reviewed Publications:}

1. Lee WD, Hurtig MB, Pilliar RM, Stanford WL, Kandel RA. Scaffold-free Engineering of Hyaline Cartilage with a Calcified Zone Using Bone Marrow Stromal Cells. Osteoarthritis and Cartilage (submitted January 2015).

2. Parreno J, Raju S, Niaki MN, Andrejevic K, Jiang A, Delve E, Kandel R. Expression of type I collagen and tenascin $\mathrm{C}$ is regulated by actin polymerization through MRTF in dedifferentiated chondrocyte. FEBS Lett. Oct 16, 2014;588(20):3677-84. PMID 25150168.

3. Ahmed N, Iu J, Chelsea E. Brown CE, Taylor DW, Kandel RA. Serum and growth factor free 3D culture system supports cartilage tissue formation by promoting collagen synthesis via Sox9-Col2a1 interaction. Tissue Engineering Part A, 20(15-16):2224-33, 2014. PMID 24606204.

4. Taylor DW, Ahmed N, Lunstrum GP, Gross AE, Diamandis EP, Kandel PA. Collagen type XII and versican are accumulated in the pericellular matrix in the early stages of tissue formation by redifferentating passaged chondrocytes. Tissue Engineering Part A, Oct 14, 2014 [Epub Ahead of Print]. PMID 25315796.

5. Mollon B, Kandel R, Chahal J, Theodoropoulos J. The clinical status of cartilage tissue regeneration in humans. Osteoarthritis Cartilage. 2013 Dec; 21(12):1824-33.

6. Vlasea M, Shanjani Y, Bothe A, Kandel RA, Toyserkani E. A combined additive manufacturing and micro-syringe deposition technique for realization of bio-ceramic 
structures with micro-scale channels. International Journal of Advanced Manufacturing Technology, 68:2261-2269, 2013.

7. Shanjani Y, Hu Y, Toyserkani E, Grynpas M, Kandel RA, Pilliar RM. Solid freeform fabrication of porous calcium polyphosphate structures for bone substitute applications: in vivo studies. J Biomed Mater Res B Appl Biomater, 101(6):972-80, Aug 2013. PMID 23529933.

8. Hu Y, Shanjani Y, Toyserkani E, Grynpas M, Wang R, Pilliar R. Porous calcium polyphosphate bone substitutes: Additive manufacturing versus conventional gravity sinter processing-Effect on structure and mechanical properties. J Biomedical Material Research B Applied Biomaterials, 101B (1): 1-8, Jan 2013. PMID 23997039.

9. Vlasea $\mathrm{M}$ and Toyserkani E. Experimental characterization and numerical modeling of a micro-syringe deposition system for dispensing sacrificial photopolymers on particulate ceramic substrates. Journal of Materials Processing Technology 213 (11):1970-1977, 2013.

\section{Book Chapter:}

1. Vlasea M, Azhari A, Basalah A, Kandel R, and Toyserkani E. Additive manufacturing for bone load bearing applications. Chapter 11 in 3D Bioprinting and Nanotechnology in Tissue Engineering and Regenerative Medicine, Edited by L.G. Zhang, J.P. Fisher, and K. Leung, Elsevier, ISBN: 9780128005477, 2015. 


\section{Abstracts:}

1. WD Lee, M.B. Hurtig, R.M. Pilliar, W.L. Stanford, R.A. Kandel. Self-assembling osteochondral-like constructs formed using bone marrow stromal cells and a bone substitute. ICRS, Chicago, May 8-11, 2015.

2. Hu Y, Grynpas M, Kandel R, Pilliar R. Sodium Carbonate-Doped Calcium Polyphosphate: Higher Mechanical Strengths and Faster Degradation Rate. CBS - Halifax, June 2014.

3. Lee WD, Hurtig MB, Pilliar RM, Stanford WL, Kandel RA. Scaffold-Free Generation of a Zone of Calcified Cartilage between Bone Marrow-derived Cartilage and the Porous Calcium Polyphosphate Substrate. CBS - Halifax, June, 2014.

4. Hu Y, Grynpas M, Kandel R, Theodoropoulos J, Pilliar R. Calcium Polyphosphate Particulate Bone Void Fillers - Rabbit Model Study. CBS - Halifax, June, 2014.

5. Pilliar R, Grynpas M, Kandel R, Hu Y, Shanjani Y, Toyserkani E. Porous Calcium Polyphosphate: A Biodegradable Bone Substitute - Mechanical \& Bone Ingrowth Characterization. Proc. of ORS 2013 Annual Meeting, San Antonio, TX, Jan 26-29, 2013.

6. Hu Y, Grynpas M, Kandel R, Pilliar R. Higher Strength Porous Calcium Polyphosphate Made by Conventional Sintering. CBS - Ottawa, June, 2013.

7. Hu Y, Grynpas M, Filiaggi M, Werner-Zwanziger U, Pilliar R. Phase Changes and Degradation of Calcium Polyphosphate . CBS - Ottawa, June, 2013.

8. Ahmed N, Taylor D, Kandel R. Role of Insulin in SOX9 Mediated COL2A1 Expression in Passaged Articular Chondrocytes. Osteoarthritis Research Society International Annual Meeting. Philadelphia, PA. April 18-21, 2013 
9. Vlasea M, Shanjani Y, Toyserkani E, Kandel R. Characterization of Calcium Polyphosphate Scaffolds with Embedded Micro-Channels for Osteochondral Tissue Replacement or Augmentation. Proc. of Biomedical Engineering Society (BMES) Atlanta, GA, USA, October 24-27, 2012.

10. Shanjani Y, Hu Y, Pilliar R, Grynpas M, Kandel R, Toyserkani E. Solid-freeformfabricated porous calcium polyphosphate structures for bone substitute uses” Proc. of ORS 2012 Annual Meeting, San Francisco, California, February 4-7, 2012.

11. Shanjani Y, Hu E, Pilliar RM, Toyserkani E. Influence of Layer Stacking Orientation on Mechanical Characteristics of Solid-Freeform-Fabricated Porous Calcium Polyphosphate Structures” Proc. of the Canadian Biomaterials Symposium, pp: 237-238, Vancouver, BC, Canada, June 2011.

12. Hu YX, St. Pierre JP, Ramsay SD, Grynpas MD, Kandel RA, Pilliar RM. The Effects of Processing Parameters on in vitro Degradation of Calcium Polyphosphate. CBS Vancouver, B.C., 2011.

\section{Presentations:}

1. Biological Joint Repair: Articulating New Therapeutic Approaches. Ontario Stem Cell Initiative Translation Talk. University of Toronto, Toronto, Ontario. January 21, 2015.

2. To Move or Not to Move: Articulating a Biological Approach to Joint Repair. Canadian Undergraduate Conference on Healthcare (CUCOH) 2014. Queen’s University, Kingston, Ontario. November 15-16, 2014.

3. Regenerative Medicine: The Next Frontier for Joint Repair. $17^{\text {th }}$ Annual LMP Graduate 
Research Conference. Chestnut Conference Center, Toronto, Ontario. March 11, 2014.

4. Comparison of Intervertebral Disc and Articular Cartilage. $11^{\text {th }}$ World Congress of the International Cartilage Repair Society. Izmir, Turkey. September 15, 2013.

5. What cells should we use for cartilage repair: stem cells or chondrocytes? Gordon Research Conference. Les Diablerets, Switzerland. April 7-12, 2013.

6. Biological Joint Repair: From Therapeutics to Joint Replacement. $38^{\text {th }}$ Annual Gallie Day. Department of Surgery, University of Toronto. Toronto, Ontario. May 4, 2012.

7. Regenerative Medicine and Joint Repair: Past, Present and Future. Peter G. Bullogh MD Honorary Lecture in Pathology. Hospital for Special Surgery. New York, New York. October 21, 2011.

\section{INVENTIONS, PATENTS AND LICENSES}

Nothing to report 


\section{REPORTABLE OUTCOMES}

1) calcium polyphosphate can be used to generate a hemi-tibial plateau and femoral condyle segment by additive manufacturing

2) the strength of the porous biomaterial can be modulated by the orientation of layering of the calcium polyphosphate

3) generated functionally graded implants with varying porosity where we can change the stacking layer thickness during the fabrication to arrive at heterogeneous porosity across the implant.

4) developed anatomically shaped medial tibial plateau and femoral condyle segmental parts suitable to use for joint repair

5) passaged chondrocytes can be induced to form cartilage tissue under serum-free conditions

6) bovine but not human chondrocytes secrete a factor(s) that induces passaged human chondrocyte redifferentiation

7) mass spectroscopic analysis has confirmed the secretion of matrix molecules and growth factors under conditions of redifferentiation

8) tissue engineered osteochondral-like constructs were generated using bone marrow derived mesenchymal stromal cells as the single cell source. The cartilage tissue and porous bone substitute substrate were formed with an interfacial zone of calcified cartilage without using any exogenous scaffolds. 
9) osteochondral-like construct had a four-fold improvement over the non-mineralized control in interfacial shear strength, which should be sufficient to prevent cartilage delamination

10) published manuscripts, presented at meetings, gave presentations.

11) submitted manuscript:

- David Lee; Mark B. Hurtig; Robert M. Pilliar; William L. Stanford; Rita A. Kandel. Scaffold-free Engineering of Hyaline Cartilage with a Calcified Zone Using Bone Marrow Stromal Cells. Submitted to Osteoarthritis and Cartilage, January 2015.

12) pending manuscripts 2 manuscripts still to be written describing manufacturing of implant and effect of age of cells on cartilage formation.

\section{OTHER ACHIEVEMENTS}

Nothing to report 


\section{REFERENCES}

1. A. N. Pollak, J. H. Calhoun, Extremity war injuries: state of the art and future directions. Introduction. J Am Acad Orthop Surg 14, viii (2006).

2. L. H. Blackbourne, The next generation of combat casualty care. J Trauma 66, S27 (Apr, 2009).

3. M. T. Mazurek, J. R. Ficke, The scope of wounds encountered in casualties from the global war on terrorism: from the battlefield to the tertiary treatment facility. J Am Acad Orthop Surg 14, S18 (2006).

4. D. C. Covey, Combat orthopaedics: a view from the trenches. J Am Acad Orthop Surg 14, S10 (2006).

5. P. J. Belmont, Jr. et al., Disease and nonbattle injuries sustained by a U.S. Army Brigade Combat Team during Operation Iraqi Freedom. Military medicine 175, 469 (Jul, 2010).

6. J. F. Spelman, S. C. Hunt, K. H. Seal, A. L. Burgo-Black, Post deployment care for returning combat veterans. Journal of general internal medicine 27, 1200 (Sep, 2012).

7. P. J. Belmont, Jr. et al., The nature and incidence of musculoskeletal combat wounds in Iraq and Afghanistan (2005-2009). Journal of orthopaedic trauma 27, e107 (May, 2013).

8. J. Geiling, J. M. Rosen, R. D. Edwards, Medical costs of war in 2035: long-term care challenges for veterans of Iraq and Afghanistan. Military medicine 177, 1235 (Nov, 2012).

9. C. B. Robbins, D. J. Vreeman, M. S. Sothmann, S. L. Wilson, N. B. Oldridge, A review of the long-term health outcomes associated with war-related amputation. Military medicine 174, 588 (Jun, 2009). 
10. R. A. Oldershaw, Cell sources for the regeneration of articular cartilage: the past, the horizon and the future. International journal of experimental pathology 93, 389 (Dec, 2012).

11. C. D. Hoemann, C. H. Lafantaisie-Favreau, V. Lascau-Coman, G. Chen, J. GuzmanMorales, The cartilage-bone interface. The journal of knee surgery 25, 85 (May, 2012).

12. A. Vahdati, D. R. Wagner, Implant size and mechanical properties influence the failure of the adhesive bond between cartilage implants and native tissue in a finite element analysis. Journal of biomechanics 46, 1554 (May 31, 2013).

13. J. D. Harris et al., Failures, re-operations, and complications after autologous chondrocyte implantation--a systematic review. Osteoarthritis and cartilage / OARS, Osteoarthritis Research Society 19, 779 (Jul, 2011).

14. W. R. Taylor et al., The medial-lateral force distribution in the ovine stifle joint during walking. Journal of orthopaedic research : official publication of the Orthopaedic Research Society 29, 567 (Apr, 2011).

15. N. K. Lee-Shee, J. P. Dickey, M. B. Hurtig, Contact mechanics of the ovine stifle during simulated early stance in gait. An in vitro study using robotics. Veterinary and comparative orthopaedics and traumatology: V.C.O.T 20, 70 (2007).

\section{APPENDICES}

Published articles attached 


\section{Elsevier Editorial System(tm) for Osteoarthritis and Cartilage Manuscript Draft}

Manuscript Number:

Title: Scaffold-free Engineering of Hyaline Cartilage with a Calcified Zone Using Bone Marrow Stromal Cells

Article Type: Manuscript

Keywords: tissue engineering; mesenchymal stromal cells; cartilage-bone interface; osteochondral constructs

Corresponding Author: Dr. Rita Kandel, MD

Corresponding Author's Institution: Mount Sinai Hospital

First Author: Whitaik David Lee, B.A.Sc.

Order of Authors: Whitaik David Lee, B.A.Sc.; Mark B Hurtig, DVM; Robert M Pilliar, PhD; William L Stanford, PhD; Rita A Kandel, MD

Abstract: Objective: In healthy joints, a zone of calcified cartilage (ZCC) provides the mechanical integration between articular cartilage and subchondral bone. Recapitulation of this architectural feature should serve to resist the constant shear force from the movement of the joint and prevent the delamination of tissue-engineered cartilage. Previous approaches to create the ZCC at the cartilagesubstrate interface have relied on strategic use of exogenous scaffolds and adhesives, which are susceptible to failure by degradation and wear. In contrast, we report a successful scaffold-free engineering of ZCC to integrate tissue-engineered cartilage and a porous biodegradable bone substitute, using sheep bone marrow stromal cells (BMSCs) as the cell source for both cartilaginous zones. Design: BMSCs were predifferentiated to chondrocytes, harvested and then grown on a porous calcium polyphosphate substrate in the presence of triiodothyronine (T3). T3 was withdrawn, and additional predifferentiated chondrocytes were placed on top of the construct and grown for 21 days. Results: This protocol yielded two distinct zones: hyaline cartilage that accumulated proteoglycans and collagen type II, and calcified cartilage adjacent to the substrate that additionally accumulated mineral and collagen type X. Constructs with the calcified interface had comparable compressive strength to native sheep osteochondral tissue and higher interfacial shear strength compared to control without a calcified zone. Conclusion: This protocol improves on the existing scaffold-free approaches to cartilage tissue engineering by incorporating a calcified zone. Since this protocol employs no xenogeneic material, it will be appropriate for use in preclinical large-animal studies.

Suggested Reviewers:

Opposed Reviewers: 


\title{
Scaffold-free Engineering of Hyaline Cartilage with a Calcified Zone Using Bone Marrow Stromal Cells
}

\author{
Whitaik David Lee, BASc ${ }^{1}$; Mark B. Hurtig, DVM ${ }^{2}$; Robert M. Pilliar, $\mathrm{PhD}^{1,3}$; \\ William L. Stanford, $\mathrm{PhD}^{1,4, *}$; Rita A. Kandel, $\mathrm{MD}^{1,5, *}$
}

\footnotetext{
${ }^{1}$ Institute of Biomaterials and Biomedical Engineering, University of Toronto; 164 College St., Toronto, Ontario M5S 3G9 Canada

${ }^{2}$ Ontario Veterinary College, University of Guelph;

50 McGulvray Lane, Guelph, Ontario N1G 2W1 Canada

${ }^{3}$ Faculty of Dentistry, University of Toronto; 124 Edward St., Toronto, Ontario M5G 1G6 Canada

${ }^{4}$ Sprott Centre for Stem Cell Research, Ottawa Hospital Research Institute \& Departments of Cellular \& Molecular Medicine and Biochemistry, Microbiology and Immunology, University of Ottawa 501 Smyth Road, Box 511. Ottawa, Ontario K1H 8L6 Canada

${ }^{5}$ Department of Pathology and Laboratory Medicine \& Lunenfeld-Tanenbaum Research Institute, Mount Sinai Hospital, University of Toronto;

600 University Ave., Toronto, Ontario M5G 1X5 Canada

* Co-corresponding authors
}

Correspondence Information:

Rita A. Kandel, MD

Dept. of Pathology and Laboratory Medicine

Mount Sinai Hospital

600 University Avenue

Toronto, Ontario M5G 1X5

Canada

Phone: +1 (416) 586-8516

Fax: +1 (416) 586-8719

Email: rkandel@mtsinai.on.ca
William L. Stanford, PhD

Sprott Centre for Stem Cell Research Ottawa Health Research Institute 501 Smyth Road, Box 511

Ottawa, Ontario K1H 8L6

Canada

Phone: +1 (613) 737-8899 x75495

Fax: +1 (613) 739-6294

Email: wstanford@ohri.ca

Running title (40 characters maximum): TE cartilage with a calcified interface 


\section{Abstract}

2 Objective: In healthy joints, a zone of calcified cartilage (ZCC) provides the mechanical

3 integration between articular cartilage and subchondral bone. Recapitulation of this

4 architectural feature should serve to resist the constant shear force from the movement of

5 the joint and prevent the delamination of tissue-engineered cartilage. Previous approaches

6 to create the ZCC at the cartilage-substrate interface have relied on strategic use of

7 exogenous scaffolds and adhesives, which are susceptible to failure by degradation and

8 wear. In contrast, we report a successful scaffold-free engineering of ZCC to integrate

9 tissue-engineered cartilage and a porous biodegradable bone substitute, using sheep bone

10 marrow stromal cells (BMSCs) as the cell source for both cartilaginous zones.

11 Design: BMSCs were predifferentiated to chondrocytes, harvested and then grown on a

12 porous calcium polyphosphate substrate in the presence of triiodothyronine (T3). T3 was

13 withdrawn, and additional predifferentiated chondrocytes were placed on top of the

14 construct and grown for 21 days.

15 Results: This protocol yielded two distinct zones: hyaline cartilage that accumulated

16 proteoglycans and collagen type II, and calcified cartilage adjacent to the substrate that

17 additionally accumulated mineral and collagen type $\mathrm{X}$. Constructs with the calcified

18 interface had comparable compressive strength to native sheep osteochondral tissue and

19 higher interfacial shear strength compared to control without a calcified zone.

20 Conclusion: This protocol improves on the existing scaffold-free approaches to cartilage

21 tissue engineering by incorporating a calcified zone. Since this protocol employs no

22 xenogeneic material, it will be appropriate for use in preclinical large-animal studies. 


\section{Introduction}

2 In a synovial joint, articular cartilage bears both compressive and shear forces to facilitate

3 weight bearing and movement. Once damaged, these mechanical forces contribute to the

4 progression of cartilage damage, resulting in loss of cartilage and subchondral bone

5 remodelling. Tissue engineering is widely regarded as a promising technology to generate

6 constructs that could replace damaged cartilage and bone [1]. A wide range of

7 osteochondral tissue engineering strategies are under investigation [2], including our

8 effort to produce biphasic constructs that consist of scaffold-free cartilage tissue formed

9 by articular chondrocytes or bone marrow stromal cells (BMSCs) on porous bone

10 substitutes (calcium polyphosphate; CPP) [3, 4]. CPP is a biodegradable ceramic with

11 excellent load-bearing and osseointegrative properties [5, 6]. In our design, ingrowth of

12 bone into CPP holds the biphasic construct in place and provide support against

13 compressive force on tissue-engineered cartilage. However, to resist shear force, a

14 mechanically competent interface between the cartilage and its substrate is needed.

15 Preventing cartilage delamination is a common challenge shared by many cartilage repair

16 strategies $[7,8]$.

18 Healthy cartilage tissue interfaces with bone in vivo through a zone of calcified

19 cartilage, which enhances mechanical integration by redistributing forces acting at the

20 interface [9]. BMSCs are suitable for creating calcified cartilage, because they can be

21 differentiated to produce cartilage tissues that mineralize their matrix [10]. While

22 chondrocytes from the deep zone of articular cartilage can also yield calcified cartilage in

23 vitro [11], BMSCs can be obtained without donor site morbidity and expanded to a large 
1 number. At the same time, in vivo studies have shown that BMSC-derived cartilage can

2 maintain their non-calcified phenotype after being implanted in a joint cavity [12, 13],

3 demonstrating the potential of BMSCs to produce both calcified and non-calcified

4 cartilage. However, to use BMSCs as a single source of cells to create cartilage tissues

5 with a calcified cartilaginous interface, limiting mineralization of BMSC-derived

6 cartilage to the interface will be crucial for the success of these implants.

8 Using BMSCs as the cell source, we report the first successful engineering of an

9 osteochondral-like construct that incorporates a zone of calcified cartilage at the

10 cartilage-CPP interface without the use of a scaffold. A stepwise culturing strategy

11 enabled the selective activation of BMSC-derived chondrocytes to induce mineralization

12 only at the interface, while avoiding mineralization in the contiguous cartilage tissue, thus

13 conferring localized calcified and hyaline zones to the engineered cartilage tissue. This

14 approach is clinically relevant as the BMSCs were expanded in media supplemented with

15 autologous serum and the construct cultured in serum-free, defined media, so this strategy

16 can be directly translated to in vivo preclinical studies.

17 


\section{Materials and methods}

2 Chondrogenic predifferentiation of BMSCs in membrane cultures

3 Sheep bone marrow stromal cells (BMSCs) were isolated and expanded from bone

4 marrow aspirates taken from ewes aged between 2 and 5 years by culturing in expansion

5 media (XM) as previously described [4] with modifications. XM consists of minimum

6 essential media $\alpha(\alpha \mathrm{MEM})$ (Wisent, St-Bruno, QC, Canada) and 10\% (v/v) autologous

7 serum. Autologous serum was generated by collecting blood from each sheep and

8 allowing them to clot for 30 minutes. BMSCs were harvested for passaging with TrypLE

9 Select (Life Technologies, Burlington, ON, Canada). BMSCs were cryopreserved after

10 first passage. After thawing, BMSCs were seeded at 5000 cells $/ \mathrm{cm}^{2}$ and further expanded

11 in monolayer for two passages.

13 To differentiate BMSCs to chondrocytes, $2.0 \times 10^{6}$ MSCs were seeded onto $12 \mathrm{~mm}$ cell

14 culture inserts $(0.2 \mu \mathrm{m}$ pore size; Millipore, Billerica, MA) and coated with $50 \mu \mathrm{g} / \mathrm{ml}$

15 collagen type IV (Sigma-Aldrich, Oakville, ON, Canada). After 6 hours, XM was

16 replaced with a defined chondrogenic media (CM) composed of high-glucose Dulbecco's

17 modified Eagle medium (hgDMEM; Life Technologies), $1 \times$ ITS + cell culture supplement

18 (BD Biosciences, Bedford, MA), 100nM dexamethasone (Sigma-Aldrich), 100 $\mu \mathrm{g} / \mathrm{mL}$

19 ascorbic acid (Sigma-Aldrich) and $10 \mathrm{ng} / \mathrm{mL}$ transforming growth factor- $\beta 3$ (TGF- $\beta 3$;

20 R\&D Systems, Minneapolis, MN). For the first 72 hours, CM was additionally

21 supplemented with $10 \mu \mathrm{M}$ blebbistatin (Cayman Chemical, Ann Arbor, MI) to inhibit

22 spontaneous detachment of cells. Media was changed every 2-3 days. After 3 weeks of

23 culture, cells (herein referred to as predifferentiated chondrocytes or PDCs) were isolated 
1 by digesting the tissue in $0.5 \%(\mathrm{w} / \mathrm{v})$ collagenase (Roche Diagnostics, Indianapolis, IN)

2 for 2 hours at $37^{\circ} \mathrm{C}$.

$4 \quad$ Preparation of porous CPP substrates with a hydroxyapatite coating

5 Cylindrical CPP rods of $4 \mathrm{~mm}$ diameter were prepared by gravity sintering $75-150 \mu \mathrm{m}$

$6 \mathrm{CPP}$ powder particles at $950^{\circ} \mathrm{C}$ as previously described [14]. Disks of $2 \mathrm{~mm}$ thickness

7 were cut from the rods and incubated in phosphate-buffered saline (PBS) at $37^{\circ} \mathrm{C}$ for 1

8 week. Then, a thin, submicron hydroxyapatite layer was deposited over the porous CPP

9 substrate using a sol-gel processing method. This hydroxyapatite layer was intended to

10 serve as a 'barrier' coating inhibiting the rate of release of CPP degradation products

11 during in vitro cartilage formation [15]. The sol was prepared with triethyl phosphite and

12 calcium nitrate tetrahydrate (Sigma-Aldrich) as previously described [16]. CPP disks

13 were dipped into the sol gel sideways for 8 seconds and withdrawn at a speed of

$1420 \mathrm{~cm} / \mathrm{min}$, air-dried for 10 minutes and annealed for 15 minutes at $210^{\circ} \mathrm{C}$. To ensure a

15 continuous barrier coating, coated disks were dipped again, annealed at $500^{\circ} \mathrm{C}$ for 20

16 minutes and gradually cooled. The coated disks were placed in Tygon tubing to create a

17 well-like structure and subsequently sterilized by $\gamma$-irradiation (2.5 MRad).

19 Optimizing the mineralizing culture condition for predifferentiated chondrocytes

20 To find a culture condition in which PDCs would form calcified cartilage tissue, $1.5 \times 10^{6}$

21 PDCs were placed on the top surface of porous CPP disks as previously described [4] and

22 cultured in a basal media composed of hgDMEM, ITS,$+ 100 \mu \mathrm{g} / \mathrm{mL}$ ascorbic acid,

$2350 \mu \mathrm{g} / \mathrm{mL}$ L-proline and 10mM $\beta$-glycerophosphate (both Sigma-Aldrich). To determine 
1 which condition would induce mineralization, $100 \mathrm{nM}$ dexamethasone, $3 \mathrm{nM}$

2 triiodothyronine (T3) or 100nM retinoic acid (all Sigma-Aldrich) were added to cultures.

3 The cultures were harvested at various times up to 21 days for further study.

$5 \quad$ Tissue culture of multiphasic constructs

6 PDCs were isolated from membrane cultures and placed on the top surface of porous CPP

7 disks $\left(1.0 \times 10^{6}\right.$ cells per disk). Cells were cultured in a media composed of hgDMEM, $1 \times$

$8 \mathrm{ITS}+, 100 \mu \mathrm{g} / \mathrm{mL}$ ascorbic acid, $50 \mu \mathrm{g} / \mathrm{mL}$ L-proline and $10 \mathrm{mM} \beta$-glycerophosphate, with

9 or without 3nM T3. T3 was withdrawn at day 4. At day 5, additional PDCs were isolated

10 and placed on top of the existing construct $\left(1.5 \times 10^{6}\right.$ cells per construct). The multi-

11 layered constructs were maintained in culture under the same culture conditions. This

12 protocol is shown in Figure 1. Constructs were harvested at 10 days for histological

13 examination and 21 days for histology, biochemical and mechanical analyses.

15 Visualization and quantification of alkaline phosphate (ALP) activity

16 To visualize the ALP activity, tissues on CPP substrates were excised and fixed in $10 \%$

17 neutral buffered formalin for 1 hour. Then, samples were incubated in 30\% (w/v) sucrose

18 overnight at $4^{\circ} \mathrm{C}$ and snap-frozen in Tissue-Tek OCT compound (Sakura Finetek,

19 Torrance, CA). $6 \mu \mathrm{m}$ cross-section cryosections were cut and mounted on silanized glass

20 slides. Sections were incubated in PBS for 15 minutes, then in azo dye (Naphthol AS-MX

21 phosphate and Fast Blue BB salt, both Sigma-Aldrich) for 10 minutes. Sections were

22 counterstained with eosin, dried and mounted with coverslips. To quantify the ALP

23 activity, cells were isolated from excised tissues by digestion in $0.5 \%$ collagenase for 2 
1 hours and lysed by sonication for 15 minutes in $0.2 \mathrm{M}$ Tris $\mathrm{pH} 7.4$ buffer. Activity levels

2 were quantified with $p$-nitrophenol phosphate (Sigma-Aldrich) and measuring the

3 absorbance at $405 \mathrm{~nm}$ against a standard curve of $p$-nitrophenol [11]. Values were

4 normalized to the lysate's DNA content (see Biochemical Analysis).

6 Micro-computed tomography $(\mu \mathrm{CT})$ imaging

7 Constructs composed of tissue and CPP were imaged using a Skyscan 1174v2 $\mu \mathrm{CT}$

8 scanner (Bruker, Belgium). Scanning was performed at $50 \mathrm{kV}$ and $800 \mu \mathrm{A}$ through a

$90.25 \mathrm{~mm}$ aluminum shield with a voxel size of $6.9 \mu \mathrm{m}$. After reconstruction, cross-

10 sectional tomographs were obtained with the software provided by the manufacturer.

12 Histological analysis and immunofluorescence

13 Constructs were fixed in $10 \%$ neutral buffered formalin overnight and infiltrated using

14 the Osteo-Bed bone embedding kit (Polysciences Inc., Warrington, PA). Sections were

15 cut and ground to $\sim 50 \mu \mathrm{m}$ thickness, stained with toluidine blue and light green and

16 visualized under light microscopy. Also, tissues were excised from the CPP substrates

17 and fixed in $10 \%$ neutral buffered formalin for 1 hour. Samples were incubated in $30 \%$

18 (w/v) sucrose overnight at $4^{\circ} \mathrm{C}$ and snap-frozen in the Tissue-Tek OCT compound. $6 \mu \mathrm{m}$

19 cross-section cryosections were cut and placed on silanized glass slides. Tissue mineral

20 and proteoglycan accumulation was visualized with von Kossa and toluidine blue stain.

21 For collagen type I visualization, sections were treated sequentially with $2.5 \mathrm{mg} / \mathrm{mL}$

22 trypsin and $25 \mathrm{mg} / \mathrm{mL}$ hyaluronidase, whereas for visualizing collagen types II and X,

23 they were treated with $2 \mathrm{mg} / \mathrm{mL}$ pepsin (all Sigma-Aldrich). Sections were then incubated 
1 with antibodies reactive with the specific collagen (Type I: CalBiolChem, La Jolla, CA;

2 type II: Labvision, Fremont, CA; type X: Sigma-Aldrich). DAPI (Life Technologies)

3 counterstain was applied to all immunostained sections.

4

5 Biochemical analysis of extracellular matrix and mineral accumulation

6 Tissues were excised from the CPP substrates and digested in $40 \mu \mathrm{g} / \mathrm{mL}$ papain (Sigma-

7 Aldrich) for 48 hours at $65^{\circ} \mathrm{C}$, and their DNA, glycosaminoglycan and hydroxyproline

8 contents were quantified as previously described [17]. To measure mineral accumulation,

9 the tissues were lyophilized, dry weights measured, and tissues were digested in $3 \mathrm{~N}$

10 hydrochloric acid at $90^{\circ} \mathrm{C}$ for 2 hours. The $\mathrm{pH}$ of samples was adjusted to 4.0 with the

11 addition of $1.5 \mathrm{M}$ acetate buffer. Phosphate content was determined using the heteropoly

12 blue assay and measuring the absorbance at $620 \mathrm{~nm}$. Calcium content was determined

13 using the o-cresolphthalein complexone assay and measuring the absorbance at 570nm.

15 Mechanical testing of multiphasic constructs

16 On day 21 of culture, the equilibrium compressive modulus of the multiphasic construct's

17 cartilaginous tissue was determined by stress relaxation testing on the Mach-1 mechanical

18 testing platform (Bio-Momentum, Laval, QC, Canada) with a $0.65 \mathrm{~mm}$-diameter indenter

19 as previously described [4]. Interfacial shear strength was determined by applying a force

20 at the interface region of these samples using a specially designed jig attached to an

21 Instron universal testing machine as previously described [15]. Using this apparatus, a

22 shear load was directly applied to the interface with a constant rate of displacement

$23(1 \mathrm{~mm} / \mathrm{min})$ until a sudden decrease in the resisting force was measured. The interface 
1 was considered to have failed at this point. Each construct was visually inspected

2 afterwards to verify that the failure did occur at the interface. The energy absorbed by the

3 interface before failure was calculated by integrating the force-displacement curve.

$5 \quad$ Statistical testing

6 Each experiment was carried out with three separate donor animals. Replicate data from

7 cells derived from a single donor animal were pooled. All experimental values are

8 expressed in mean $\pm 95 \%$ confidence interval. For time-course experiments, two-way

9 analysis of variance (ANOVA) was used to analyze the effects of culture conditions and

10 time. Biochemical and biomechanical data were evaluated using one-way ANOVA and

11 Tukey post hoc testing.

12 


\section{Results}

2 Generation of calcified cartilage at the CPP interface with predifferentiated

3 chondrocytes (PDCs)

4 To engineer a multiphasic construct that incorporates a zone of calcified cartilage at the

5 cartilage-CPP interface using PDCs, we first sought to establish a culture condition in

6 which PDCs could be cultured on porous CPP substrates to form mineralized cartilage

7 tissue. Triiodothyronine (T3) [18], retinoic acid (RA) [19] and dexamethasone [10] have

8 all been identified as potential inducers of chondrocyte mineralization in vitro. We

9 cultured PDCs on CPP substrates with either 3nM T3 or 100 $\mu$ M RA treatment in the

10 presence or absence of dexamethasone for 1 week, and then quantified the alkaline

11 phosphate activity of the cells as an indicator of chondrocyte mineralization potential [20,

12 21]. ALP activity was the highest in PDCs cultured in the absence of dexamethasone with

13 the T3 treatment (Figure 2A). While ALP activity was also increased with the RA

14 treatment as well, continued culture did not yield cartilaginous tissues on CPP after 3

15 weeks (data not shown). Therefore, treatment of PDCs with T3 was selected for further

16 study.

18 When $2 \times 10^{6}$ PDCs were cultured on CPP substrates for 3 weeks, the mineralized

19 zone was found in the top of the tissue (Figure 2B). Between this mineralized zone and

20 the CPP substrate was an intermediary zone of non-mineralized cartilage, composed of

21 cells with round morphology and extracellular matrix rich in proteoglycans. Changing the

22 concentration of $\mathrm{T} 3$ treatment did not change the location of the mineralized zone (data

23 not shown). However, when the number of initially seeded PDCs was decreased, the 
1 distance between the CPP-cartilage interface and the zone of mineralized cartilage also

2 decreased (Figure 2C, arrows; Figure 2D). At an initial seeding of $1 \times 10^{6}$ PDCs, the zone

3 of mineralized cartilage formed consistently at the hydroxyapatite-coated CPP substrate

4 interface.

6 Short-term treatment of PDCs with T3 was sufficient to maintain ALP activity

7 ALP activity levels of PDCs were characterized over a 2-week culture period (Figure 3).

8 In the presence of continuous T3 treatment, ALP activity level was significantly

9 increased by day 4 and maintained at that level up to day 14 ( $p=0.334$ between days 4 to

1014 by 1-way ANOVA). Interestingly, when the T3 treatment was withdrawn at day 4, the

11 ALP activity level did not change, and was comparable to cells treated continuously with

12 T3 for the rest of the culture period ( $p=0.887$ by 2 -way ANOVA). This data

13 demonstrated that T3 was expendable after day 4.

15 T3-treated PDCs did not induce ALP activity in non-T3-treated PDCs

16 Based on this observation, a two-stage culture protocol was developed (Figure 1). To

17 grow an interfacial zone of mineralized cartilage, $1.0 \times 10^{6}$ PDCs were first seeded on

18 porous CPP substrates, which was the number of cells that had been previously

19 determined to be optimal for creating the mineralized cartilage at the interface, and

20 cultured in the presence of T3 (Figure 2C \& 2D). T3 was withdrawn at 4 days of culture,

21 then $1.5 \times 10^{6}$ PDCs were seeded on top of the existing tissue one day later and cultured in

22 the presence of $\beta$-glycerophosphate for up to 21 days to generate a zone of non-

23 mineralized cartilage. To confirm that the T3-treated cells would not stimulate the 
1 overlaid, non-T3-treated cells to also mineralize, the tissues on CPP were harvested 5

2 days after the addition of the top layer of cells (10 days of culture in total) and

3 cryosectioned. Histological analysis demonstrated that ALP activity was present only in

4 the calcifying bottom layer (Figure 4), demonstrating the lack of mineralization potential

5 in the top layer even though $\beta$-glycerophosphate was present.

$7 \quad$ Characterization of the multiphasic constructs

8 Constructs were grown for 21 days using the two-stage culture protocol with or without

9 the 4-day T3 treatment of the interfacial layer and characterized using various methods.

10 Histological examination of cartilage tissue on the CPP showed two tissue zones. The

11 upper zone of non-mineralized cartilage was hyaline cartilage, rich in type II collagen and

12 proteoglycan (Figure 5A, B, D). The interfacial zone was calcified cartilage, the mineral

13 staining with von Kossa staining and the extracellular matrix staining with toluidine blue

14 and type X collagen (Figure 5A, B, E). Ingrowth of tissue into upper parts of the porous

15 CPP substrate was also observed, with the mineral clusters directly in contact with the

16 CPP particles (Figure 5A). No type I collagen was detected in either zone (Figure 5C). In

17 contrast, control constructs not treated with T3 did not show a bizonal composition as

18 there was no zone of calcified cartilage, and there was an uneven distribution of

19 extracellular matrix (Figure 5F-I). Tissues on T3-treated constructs accumulated less

20 proteoglycans (Figure 6A) and collagens (Figure 6B) compared to those on untreated

21 constructs, but showed amounts comparable to native sheep cartilage. Tissues on T3-

22 treated constructs accumulated calcium and phosphate in keeping with the presence of

23 calcification, while untreated constructs did not (Figure 6C). 
2 The mechanical properties of the T3-treated and untreated constructs were

3 characterized by measuring the equilibrium compressive modulus and the interfacial

4 shear strength. T3-treated tissues had a comparable equilibrium compressive modulus to

5 that of osteochondral tissue obtained from native sheep femoral condyles (Figure 7A, $p=$

6 0.15). The untreated constructs had a significantly higher equilibrium compressive

7 modulus as compared to that of the T3-treated tissues, possibly due to their greater

8 extracellular matrix accumulation (Figure 6A, 6B). However, T3-treated constructs could

9 withstand a stronger shear force (Figure 7B) and absorb more energy (Figure 7C) than

10 untreated constructs. This demonstrates that the mineralized tissue at the interface (Figure

$115 \mathrm{~A}$ ) enhanced the interfacial shear strength.

12

13

14

15 


\section{Discussion}

2 A mechanically competent interface between the cartilage and its substrate is required to

3 resist the shear force generated by the movement of the joint. Previous studies have

4 sought to recreate the zone of calcified cartilage that exists between hyaline cartilage and

5 bone to reinforce this interface with limited success [22]. To overcome this problem, we

6 successfully tissue engineered osteochondral-like constructs with BMSCs as the single

7 cell source, in which cartilage tissue and a porous bone substitute substrate were formed

8 with an interfacial zone of calcified cartilage without using any exogenous scaffolds. The

9 two-stage culture protocol enabled the selective stimulation of predifferentiated

10 chondrocytes to form calcified cartilage only at the interface. This zone of calcified

11 cartilage, in addition to mineral, also accumulated collagen type X, collagen type II and

12 proteoglycans. These features were seen only in the part of the tissue that was treated

13 with mineralization-inducing T3, which was adjacent to (and integrated with) the porous

14 CPP substrate. The interfacial shear strength of constructs with the calcified cartilaginous

15 interface was increased significantly compared to those without it, functionally validating

16 its intended effect. The hyaline quality of non-mineralized cartilage was demonstrated by

17 the accumulation of collagen type II and the lack of mineral or collagen type I.

19 Thus far, approaches to induce selective mineralization of BMSC-derived

20 cartilage and create this interface include delivery of bioactive signals either locally via

21 microspheres [23] or by using gradients generated by a bioreactor [24] to undifferentiated

22 BMSCs seeded in scaffolds. In another approach, BMSCs predifferentiated to

23 chondrocytes and osteoblasts are seeded into appropriate locations within scaffolds [25, 
$126]$. For both strategies, use of exogenous scaffolds is necessary, which has the potential

2 to hinder host integration and tissue regeneration [27]. Matching the rate of scaffold

3 degeneration to the rate of tissue accumulation remains a challenge when employing

4 scaffolds [22, 28]. Synthetic materials also accumulate wear over time, and may provide

5 a focal point for shear failure if not replaced with new tissue. On the other hand, if the

6 rate of scaffold degradation exceeds the rate of cellular remodelling, the mechanical

7 integrity is compromised and the construct would fail under load. Scaffold-free

8 approaches obviates this issue altogether; however, an alternative strategy to specify the

9 location of the calcified interface was needed.

11 The development of our two-stage culture protocol was first enabled by the

12 observation that BMSC-derived PDCs formed mineralized cartilage at the tissue's

13 superficial aspect with mineral-inducing stimulus. Given that T3 was delivered to the

14 tissue through culture media, and that the location of calcified cartilage did not change

15 with tissue thickness, this effect was likely due to T3 diffusion into the tissue. As there

16 was no scaffold present, selective delivery of $\mathrm{T} 3$ to the interface was difficult to achieve

17 with media alone. To circumvent this, we seeded a small number of cells on the CPP in

18 the presence of T3. This generated thin cartilaginous tissue, forcing mineralization to take

19 place juxtaposed to the porous CPP substrate. This enabled the subsequent formation of a

20 continuous layer of non-mineralized cartilage formed by the same cell type in the absence

21 of T3. The presence of collagen types II, $\mathrm{X}$ and proteoglycans in the calcified interface,

22 as well as the absence of collagen type I, is an important finding in our study. This 
1 demonstrates that the interface is cartilaginous, not osseous, successfully recapitulating

2 characteristics of the native articular cartilage-subchondral bone interface.

$4 \quad$ Histology showed that the layer of non-mineralized cartilage successfully fused to

5 the T3-treated tissue underneath. Interestingly, fissures were observed if the intended

6 interfacial tissue (i.e., the first layer) was not treated with T3, and the total thickness of

7 cartilage tissues on these constructs was increased, with an uneven distribution of the

8 extracellular matrix. There are two possible explanations for these results. One is that the

9 manner that the interfacial layer grows may affect integrations, as BMSC-derived

10 chondrocytes share many characteristics with proliferating cartilage [29] that grows

11 appositionally. In development, Indian hedgehog, FGF and BMP signaling pathways

12 regulate this appositional growth[30]; however, in an in vitro system, absence of such

13 complex regulation may possibly allow the interfacial layer to expand laterally as well.

14 The presence of tubing around the constructs prevented lateral expansion by confinement,

15 which could have resulted in lateral mechanical stress and caused the tissue to buckle and

16 shear. This was not the case for T3-stimulated interfacial layer, however, whose

17 behaviour may more resemble terminal hypertrophic chondrocytes, as evidenced by their

18 mineralized matrix. Terminal hypertrophic chondrocytes do not proliferate and eventually

19 undergo apoptosis upon vascularization of matrix and bone formation [31]. Alternatively,

20 the T3 treatment may have affected tissue fusion. Pellets of human mesenchymal stromal

21 cells undergoing chondrogenesis have been shown to accumulate tenascin along the outer

22 surface that prevented them from fusing with one another [32]. The fissure between the 
1 two layers of non-mineralizing cartilage may stem from a similar boundary-setting

2 phenomenon, whether driven by tenascin accumulation or otherwise.

$4 \quad$ Sheep gait studies estimate the maximum anterioposterior shear stress in a stifle

5 joint to be about $500 \mathrm{kPa}[33,34]$, which is equivalent to about $9 \mathrm{~N}$ of peak shear force in

6 our study. However, using our methodology the peak shear force of native cartilage

7 interface could not be measured, as it withstood the maximum load $(80 \mathrm{~N})$ without shear

8 failure. In healthy cartilage collagen fibrils run orthogonally through the tidemark plane,

9 which would confer a high resistance against failure in our mode of study. Although our

10 multiphasic construct possesses inferior interfacial shear strength compared to articular

11 cartilage, it still represented a four-fold improvement over the non-mineralized control,

12 and sheep gait studies suggest that this improvement is adequate for preventing

13 delamination.

15 Importantly, this protocol can be expanded to generate large, anatomically shaped

16 osteochondral constructs as replacements for damaged joint tissues. Protocols have been

17 developed to generate $\sim 10^{8}$ BMSCs with chondrogenic potential in a good manufacturing

18 practices-complaint manner [35, 36], which can be used to generate sufficiently large

19 numbers of chondrocytes to cover a large articular surface. Using the expansion

20 technique discussed in this study, we have successfully obtained $3 \times 10^{8}$ BMSCs from a

21 single sheep bone marrow aspirate within 3 passages (data not shown). We have

22 previously shown that CPP powders can be used in additive manufacturing methods to

23 generate substrates of required shape and size [37]. CPP formed in this way exhibited the 
1 same degree of osseointegration after implantation as those produced conventionally [38].

2 As such, preclinical studies are warranted to determine if these osteochondral-like

3 constructs with a mineralized cartilaginous interface could repair joint defects in vivo.

\section{Conclusion}

6 Using BMSCs as a single cell source, an osteochondral-like construct comprised of

7 hyaline cartilage and porous bone substitute was created with a zone of calcified cartilage

8 interfacing them. The novel, two-step cell culture strategy employed no exogenous

9 scaffolds, unlike comparable existing technologies for osteochondral construct

10 generation. The presence of calcified cartilage increased the shear load that the construct

11 could withstand at the interface. As no xenogeneic material was used at any point during

12 culture, this strategy can be directly translated into preclinical studies, which will

13 determine if these constructs can repair joint defects in vivo. 


\section{Acknowledgments}

2 We thank Eugene $\mathrm{Hu}$ for preparing the porous CPP substrates. We also thank Dr. Jian

3 Wang and Nancy Valiquette for their technical assistance. This work was supported by

4 the United States Department of Defense (to R.A.K., W81XWH-10-1-0787) and the

5 Natural Sciences and Engineering Research Council of Canada (to W.L.S., RGPIN

6 293170-11). W.L.S. is supported by a Canadian Research Chair.

\section{Author Contributions}

9 Study conception and design: WDL, WLS, RAK.

10 Provision of study materials: MBH, RMP.

11 Analysis and interpretation of data: WDL, RMP, RAK.

12 Drafting of article: WDL, WLS, RAK.

13 Critical revision of the article: WDL, WLS, RAK.

14 Final approval of the article: WDL, WLS, RAK.

\section{Role of the Funding Sources}

17 None of the listed bodies played any role in this study.

\section{Conflict of Interest}

20 No competing financial interests exist for any of the listed authors. 


\section{References}

2 1. Swieszkowski W, Tuan BHS, Kurzydlowski KJ, Hutmacher DW. Repair and

3 regeneration of osteochondral defects in the articular joints. Biomolecular

$4 \quad$ engineering 2007; 24: 489-495.

5 2. Shimomura K, Moriguchi Y, Murawski CD, Yoshikawa H, Nakamura N.

6 Osteochondral Tissue Engineering with Biphasic Scaffold: Current Strategies and

$7 \quad$ Techniques. Tissue Eng Part B Rev 2014; 20: 468-476.

8 3. Kandel RA, Grynpas M, Pilliar R, Lee J, Wang J, Waldman S, et al. Repair of

9 osteochondral defects with biphasic cartilage-calcium polyphosphate constructs in

10 a sheep model. Biomaterials 2006; 27: 4120-4131.

11 4. Lee WD, Hurtig MB, Kandel RA, Stanford WL. Membrane culture of bone

12 marrow stromal cells yields better tissue than pellet culture for engineering

13 cartilage-bone substitute biphasic constructs in a two-step process. Tissue

14 engineering. Part C, Methods 2011; 17: 939-948.

15 5. Grynpas MD, Pilliar RM, Kandel RA, Renlund R, Filiaggi M, Dumitriu M.

16 Porous calcium polyphosphate scaffolds for bone substitute applications in vivo

$17 \quad$ studies. Biomaterials 2002; 23: 2063-2070.

18 6. Pilliar RM, Kandel RA, Grynpas MD, Hu Y. Porous calcium polyphosphate as

19 load-bearing bone substitutes: in vivo study. J Biomed Mater Res B Appl

$20 \quad$ Biomater 2013; 101: 1-8.

$217 . \quad$ Vahdati A, Wagner DR. Implant size and mechanical properties influence the

22 failure of the adhesive bond between cartilage implants and native tissue in a

23 finite element analysis. J Biomech 2013; 46: 1554-1560. 
1 8. Harris JD, Siston RA, Brophy RH, Lattermann C, Carey JL, Flanigan DC.

2 Failures, re-operations, and complications after autologous chondrocyte

3 implantation--a systematic review. Osteoarthritis Cartilage 2011; 19: 779-791.

$49 . \quad H o e m a n n$ CD, Lafantaisie-Favreau C-H, Lascau-Coman V, Chen G, Guzmán-

$5 \quad$ Morales J. The cartilage-bone interface. The journal of knee surgery 2012; 25: 85-

697.

7 10. Mueller MB, Tuan RS. Functional characterization of hypertrophy in

8 chondrogenesis of human mesenchymal stem cells. Arthritis and rheumatism

$92008 ; 58: 1377-1388$.

10 11. Allan KS, Pilliar RM, Wang J, Grynpas MD, Kandel RA. Formation of biphasic

11 constructs containing cartilage with a calcified zone interface. Tissue engineering 12 2007; 13: 167-177.

13 12. Zscharnack M, Hepp P, Richter R, Aigner T, Schulz R, Somerson J, et al. Repair 14 of chronic osteochondral defects using predifferentiated mesenchymal stem cells

15 in an ovine model. The American journal of sports medicine 2010; 38: 1857-

$16 \quad 1869$.

17 13. Shimomura K, Moriguchi Y, Ando W, Nansai R, Fujie H, Hart DA, et al.

18 Osteochondral Repair Using a Scaffold-Free Tissue-Engineered Construct

19 Derived from Synovial Mesenchymal Stem Cells and a Hydroxyapatite-Based

$20 \quad$ Artificial Bone. Tissue Eng Part A 2014.

21 14. Waldman SD, Grynpas MD, Pilliar RM, Kandel RA. Characterization of

22 cartilagenous tissue formed on calcium polyphosphate substrates in vitro. Journal

23 of biomedical materials research 2002; 62: 323-330. 
1 15. St-Pierre J-P, Gan L, Wang J, Pilliar RM, Grynpas MD, Kandel RA. The

2 incorporation of a zone of calcified cartilage improves the interfacial shear

3 strength between in vitro-formed cartilage and the underlying substrate. Acta

$4 \quad$ biomaterialia 2012; 8: 1603-1615.

5 16. Gan L, Pilliar R. Calcium phosphate sol-gel-derived thin films on porous-surfaced

6 implants for enhanced osteoconductivity. Part I: Synthesis and characterization.

$7 \quad$ Biomaterials 2004; 25: 5303-5312.

8 17. Taylor DW, Ahmed N, Gan L, Gross AE, Kandel RA. Proteoglycan and collagen

9 accumulation by passaged chondrocytes can be enhanced through side-by-side

10 culture with primary chondrocytes. Tissue engineering. Part A 2010; 16: 643-651.

11 18. Alini M, Kofsky Y, Wu W, Pidoux I, Poole AR. In serum-free culture thyroid

12 hormones can induce full expression of chondrocyte hypertrophy leading to

13 matrix calcification. Journal of bone and mineral research : the official journal of

14 the American Society for Bone and Mineral Research 1996; 11: 105-113.

15 19. Iwamoto M, Shapiro IM, Yagami K, Boskey AL, Leboy PS, Adams SL, et al.

16 Retinoic acid induces rapid mineralization and expression of mineralization-

17 related genes in chondrocytes. Experimental cell research 1993; 207: 413-420.

18 20. Miao D, Scutt A. Histochemical localization of alkaline phosphatase activity in

19 decalcified bone and cartilage. J Histochem Cytochem 2002; 50: 333-340.

20 21. Jiang J, Leong NL, Mung JC, Hidaka C, Lu HH. Interaction between zonal

21 populations of articular chondrocytes suppresses chondrocyte mineralization and

22 this process is mediated by PTHrP. Osteoarthritis Cartilage 2008; 16: 70-82. 
1 22. Huey DJ, Hu JC, Athanasiou KA. Unlike bone, cartilage regeneration remains

$2 \quad$ elusive. Science 2012; 338: 917-921.

3 23. Dormer NH, Singh M, Wang L, Berkland CJ, Detamore MS. Osteochondral

$4 \quad$ interface tissue engineering using macroscopic gradients of bioactive signals.

$5 \quad$ Annals of biomedical engineering 2010; 38: 2167-2182.

6 24. Chen K, Ng KS, Ravi S, Goh JC, Toh SL. In vitro generation of whole

7 osteochondral constructs using rabbit bone marrow stromal cells, employing a

8 two-chambered co-culture well design. J Tissue Eng Regen Med 2013.

9 25. Cheng H-W, Luk KDK, Cheung KMC, Chan BP. In vitro generation of an

10 osteochondral interface from mesenchymal stem cell-collagen microspheres.

$11 \quad$ Biomaterials 2011; 32: 1526-1535.

12 26. Grayson WL, Bhumiratana S, Grace Chao PH, Hung CT, Vunjak-Novakovic G.

13 Spatial regulation of human mesenchymal stem cell differentiation in engineered

14 osteochondral constructs: effects of pre-differentiation, soluble factors and

15 medium perfusion. Osteoarthritis and cartilage / OARS, Osteoarthritis Research

$16 \quad$ Society 2010; $18: 714-723$.

17 27. Castro NJ, Hacking SA, Zhang LG. Recent progress in interfacial tissue

18 engineering approaches for osteochondral defects. Annals of biomedical

19 engineering 2012; 40: 1628-1640.

20 28. Dhote V, Vernerey FJ. Mathematical model of the role of degradation on matrix

21 development in hydrogel scaffold. Biomech Model Mechanobiol 2014; 13: 167-

22183. 
1 29. Dickhut A, Pelttari K, Janicki P, Wagner W, Eckstein V, Egermann M, et al.

2 Calcification or dedifferentiation: requirement to lock mesenchymal stem cells in

3 a desired differentiation stage. Journal of cellular physiology 2009; 219: 219-226.

4 30. Kronenberg HM. Developmental regulation of the growth plate. Nature 2003;

$5 \quad 423: 332-336$.

6 31. Farnum CE, Wilsman NJ. Cellular turnover at the chondro-osseous junction of

$7 \quad$ growth plate cartilage: analysis by serial sections at the light microscopical level.

$8 \quad$ J Orthop Res 1989; 7: 654-666.

9 32. Bhumiratana S, Eton RE, Oungoulian SR, Wan LQ, Ateshian GA, Vunjak-

10 Novakovic G. Large, stratified, and mechanically functional human cartilage

11 grown in vitro by mesenchymal condensation. Proc Natl Acad Sci U S A 2014;

$12 \quad 111: 6940-6945$.

13 33. Taylor WR, Poepplau BM, König C, Ehrig RM, Zachow S, Duda GN, et al. The

14 medial-lateral force distribution in the ovine stifle joint during walking. Journal of

15 orthopaedic research : official publication of the Orthopaedic Research Society

$16 \quad 2011 ; 29: 567-571$.

17 34. Lee-Shee NK, Dickey JP, Hurtig MB. Contact mechanics of the ovine stifle

18 during simulated early stance in gait. An in vitro study using robotics. Vet Comp

19 Orthop Traumatol 2007; 20: 70-72.

20 35. Chase LG, Yang S, Zachar V, Yang Z, Lakshmipathy U, Bradford J, et al.

21 Development and characterization of a clinically compliant xeno-free culture

22 medium in good manufacturing practice for human multipotent mesenchymal

23 stem cells. Stem Cells Transl Med 2012; 1: 750-758. 
1 36. Gottipamula S, Muttigi MS, Chaansa S, Ashwin KM, Priya N, Kolkundkar U, et

2 al. Large-scale expansion of pre-isolated bone marrow mesenchymal stromal cells

3 in serum-free conditions. J Tissue Eng Regen Med 2013.

4 37. Shanjani Y, De Croos JN, Pilliar RM, Kandel RA, Toyserkani E. Solid freeform

5 fabrication and characterization of porous calcium polyphosphate structures for

6 tissue engineering purposes. J Biomed Mater Res B Appl Biomater 2010; 93: 510-

$7 \quad 519$.

8 38. Shanjani Y, Hu Y, Toyserkani E, Grynpas M, Kandel RA, Pilliar RM. Solid

9 freeform fabrication of porous calcium polyphosphate structures for bone

10 substitute applications: in vivo studies. J Biomed Mater Res B Appl Biomater

$11 \quad 2013 ; 101: 972-980$.

12

13 


\section{Figure Legends}

2 Figure 1. Line diagram of the tissue culture protocol used for forming the scaffold-free

3 multiphasic osteochondral construct.

5 Figure 2. Predifferentiated chondrocytes (PDCs) formed cartilaginous tissues on porous

6 CPP substrates with a zone of mineralized cartilage. (A) ALP activity of PDCs cultured

7 for 7 days with 3nM T3 or 100nM RA in the presence or absence of dexamethasone. $n=3$.

8 (B) Von Kossa and toluidine blue-stained sections of cartilaginous tissue formed by

9 culturing $2 \times 10^{6}$ PDCs on CPP substrate with 3 nM T3 for 3 weeks, showing mineralized

10 zone occurring at the superficial aspect of the cartilage. Asterisk denotes the original

11 location of the substrate. Scale bar $=200 \mu \mathrm{m}$. (C-D) Cross-sectional $\mu$ CT tomographs (C)

12 and histology (D) of 3-week-old constructs with decreasing numbers of seeded cells:

$132 \times 10^{6}$ (left), $1.5 \times 10^{6}$ (middle) and $1.0 \times 10^{6} \mathrm{PDCs}$ (right). Arrows indicate the separation

14 between the mineralized zone and the substrate. Scale bar $=1 \mathrm{~mm}(\mathrm{C})$ or $200 \mu \mathrm{m}(\mathrm{D})$.

16 Figure 3. Continued T3 treatment was not necessary for PDCs to maintain their capacity

17 to express ALP. $1 \times 10^{6}$ PDCs were cultured on CPP with $3 \mathrm{nM}$ T3 in the absence of

18 dexamethasone; $\mathrm{T} 3$ treatment was discontinued on day 4 (squares). $n=3 ; * \mathrm{p}<0.05$; $\mathrm{ns}=$

19 not significant between individual days.

21 Figure 4. Layer of PDCs treated with T3 did not induce the overlaid layer of non-treated

22 PDCs to activate ALP activity. $1 \times 10^{6}$ cells were cultured on CPP with (A) or without (B)

$233 \mathrm{nM}$ T3 for 4 days. On day $5,1.5 \times 10^{6}$ cells were seeded on top of these cells/tissue and 
1 cultured without T3. Cryosections of tissues from day 10 multiphasic constructs were

2 stained for ALP activity. The blue staining indicative of ALP was seen only in the

3 mineralizing layer (arrow). Asterisk denotes the location of where the CPP substrate

4 would have been located prior to its removal, scale bar $=200 \mu \mathrm{m}$.

6 Figure 5. A two-step culture protocol of PDCs on CPP substrates produced cartilage

7 tissue with a mineralized zone at the interface. (A) Day 21 constructs with 4-day T3

8 treatment were processed uncalcified, sectioned and stained to reveal the cartilaginous

9 (purple), mineral (green) and CPP particles (brown). (B-K) Tissues were excised from the

10 CPP substrate and examined histologically for the distribution of mineral and

11 proteoglycan accumulation $(B, G)$, and by immunofluorescent staining for the

12 accumulation of different types of collagen (D-F, I-K). Constructs with T3-treated

13 interfacial layer are denoted as $+\mathrm{T} 3$, and untreated (no T3) control constructs are denoted

14 as $-\mathrm{T} 3$. Scale bars $=200 \mu \mathrm{m}$.

16 Figure 6. Accumulation of extracellular matrix in $\mathrm{T}_{3}$-treated $(+\mathrm{T} 3)$ tissues was less

17 compared to those on untreated (-T3) tissues, but comparable to native articular cartilage.

18 Accumulated proteoglycans (A) and total collagen (B) were quantified and normalized by

19 their DNA content. Accumulation of mineral in tissue was quantified by measuring the

20 calcium and phosphate contents, and were normalized by the tissue dry weight (C).

21 "Native" denotes data from sheep cartilage explants of femoral condyles. In (A),

22 difference between $+\mathrm{T} 3$ and Native is not statistically significant.

$23 n=6 . \dagger p<0.05, * p<0.01$ 
1 Figure 7. T3-treated constructs (+T3) exhibited comparable compressive strength as

2 native sheep osteochondral explants and stronger shear strength than untreated constructs

3 (-T3). (A) Compressive modulus was measured by stress relaxation test with a $0.65 \mathrm{~mm}-$

4 diameter indenter situated at the centre of the tissue. $(\mathrm{B}, \mathrm{C})$ A shear load was applied to

5 the cartilage-CPP interface until failure. All CPP substrates were identical in shape. The

6 interfacial shear strength testing could not be performed on osteochondral explants due to

$7 \quad$ slippage of the cartilage tissue. In (A), difference between $+\mathrm{T} 3$ and Native is not

8 statistically significant. $n=9$ for constructs and 6 for explants. $\uparrow p<0.05, * p<0.01$. 
Click here to download high resolution image

Sheep bone marrow aspirate<smiles>C1CC2C3CC2C2(CCCC2)C3C1</smiles>

Isolation

Expansion

Cryopreservation
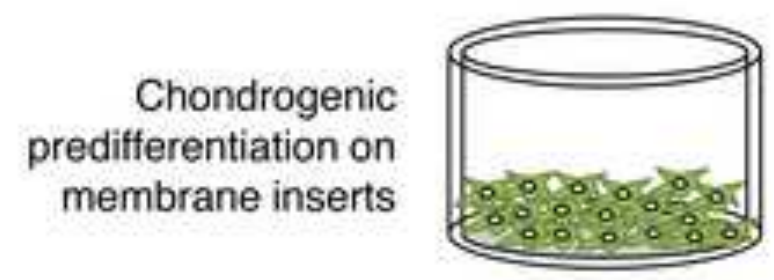

DMEM, ITS+

Dex, TGF- $\beta 3$

expansion culture

aMEM +
Substrates

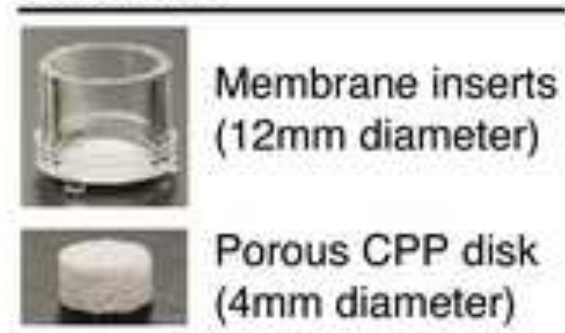

Membrane inserts

Porous CPP disk

(4mm diameter)

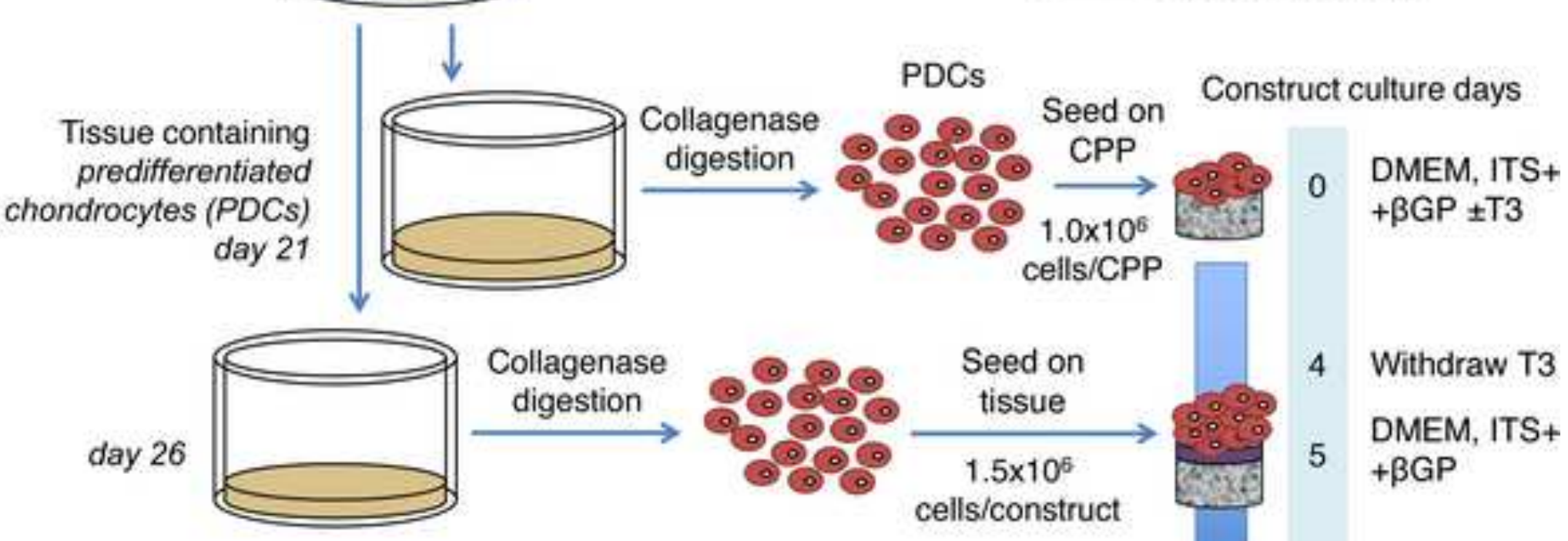

Hyaline cartilage Calcified cartilage CPP substrate

\section{Expanded BMSCs}

r

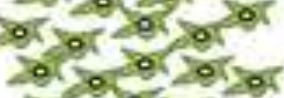

or o or zor

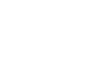


Click here to download high resolution image
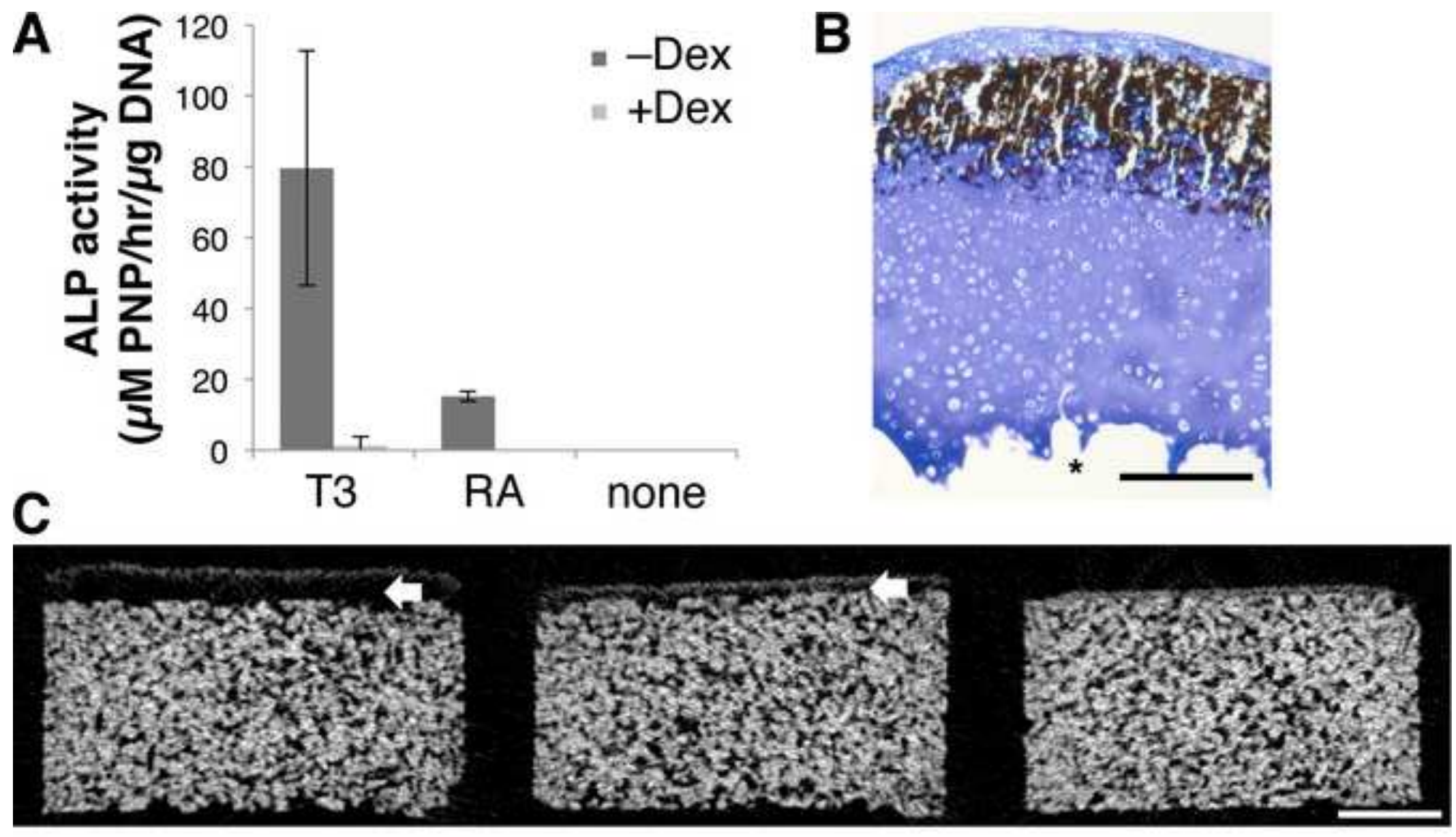

\section{D}
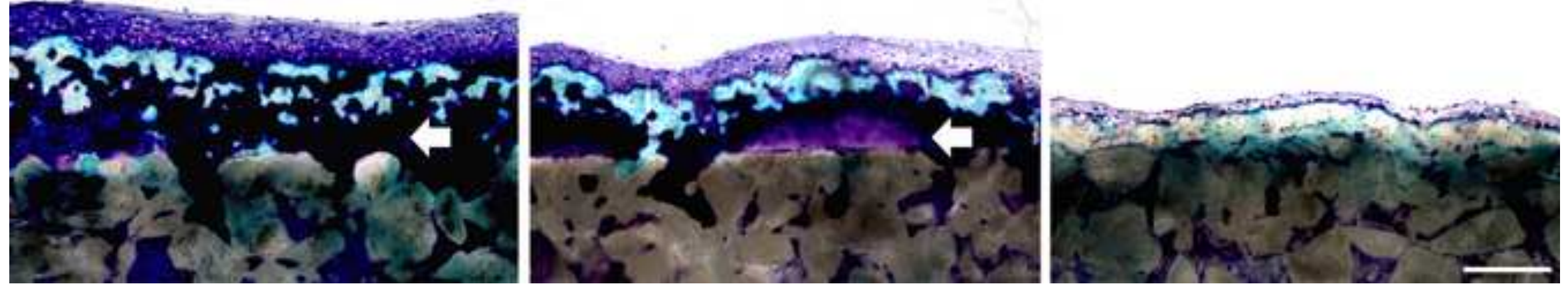


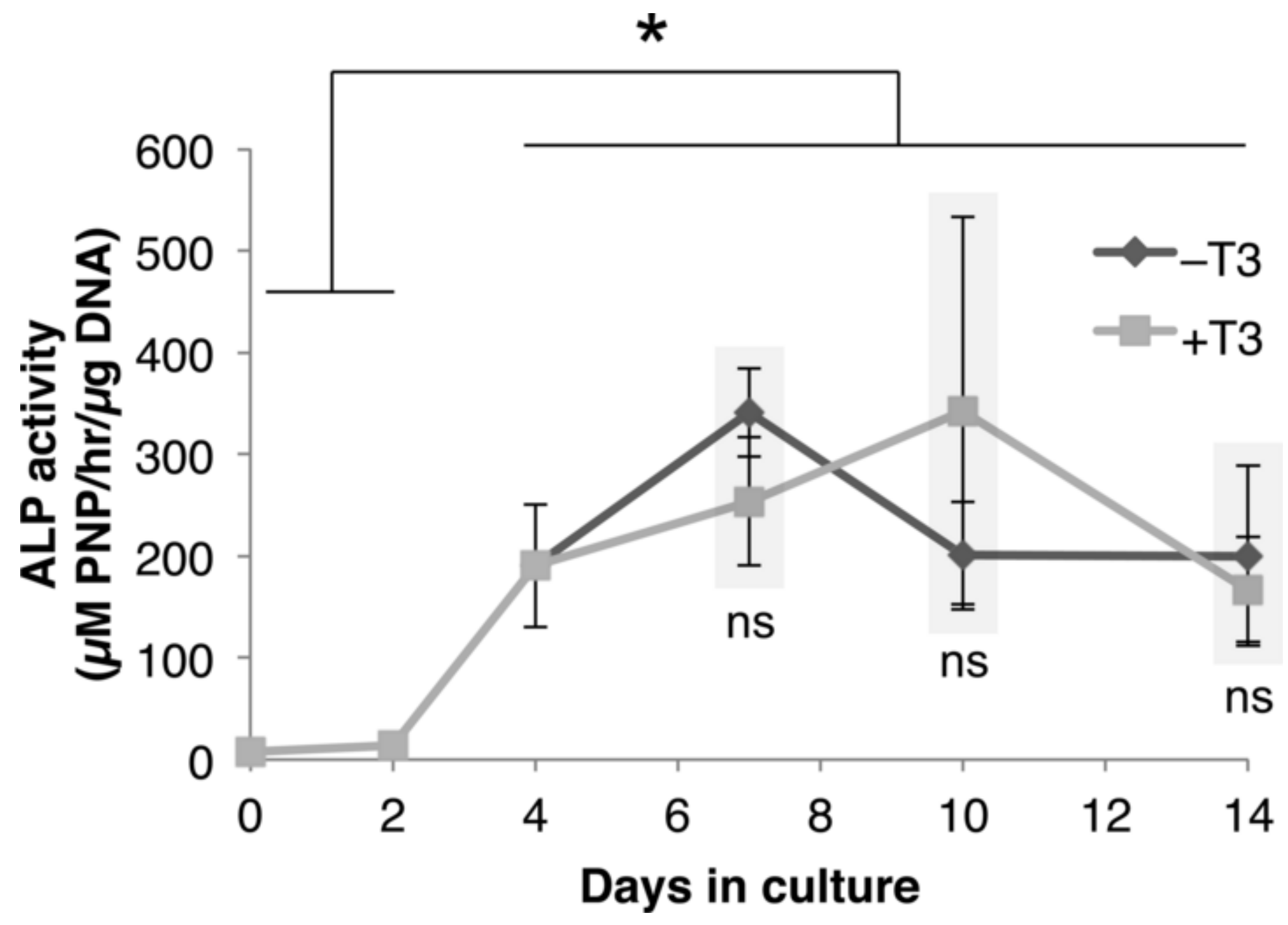


Click here to download high resolution image
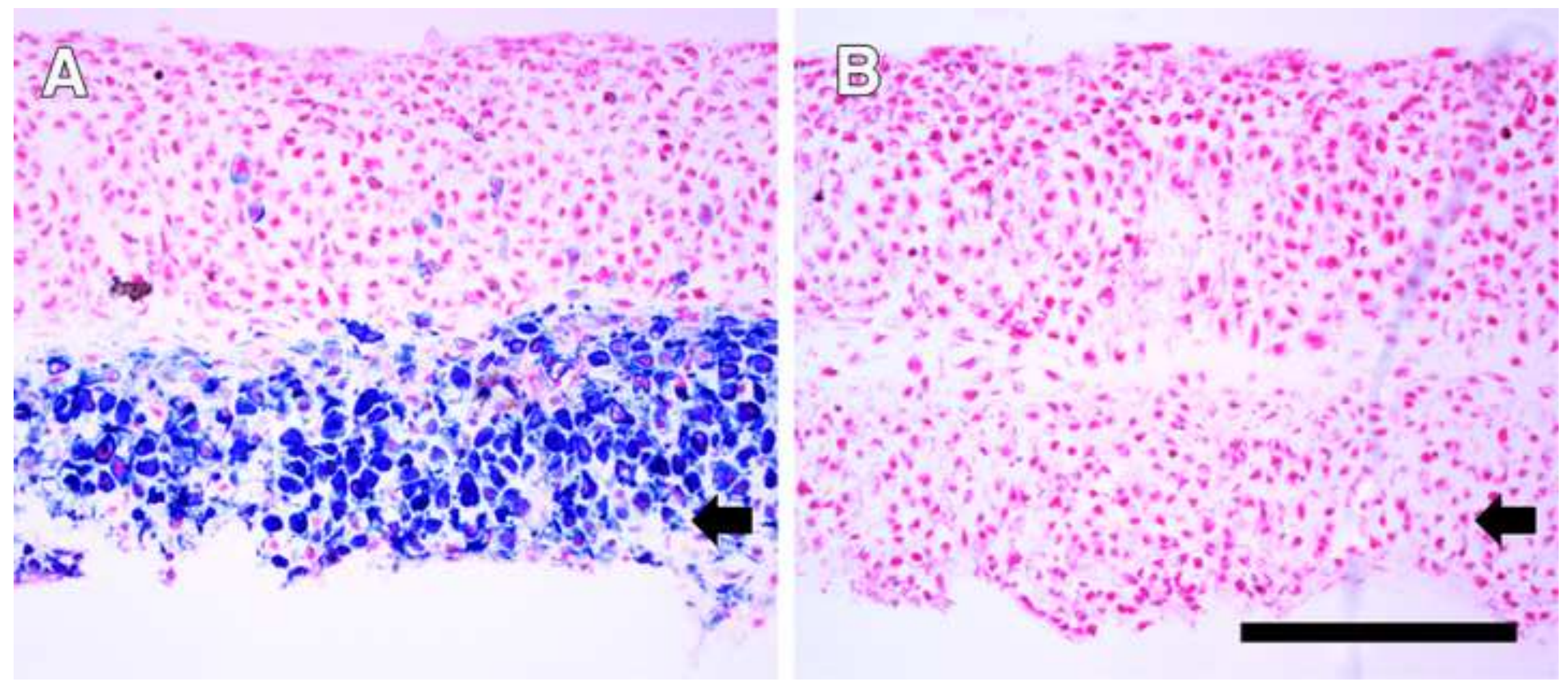


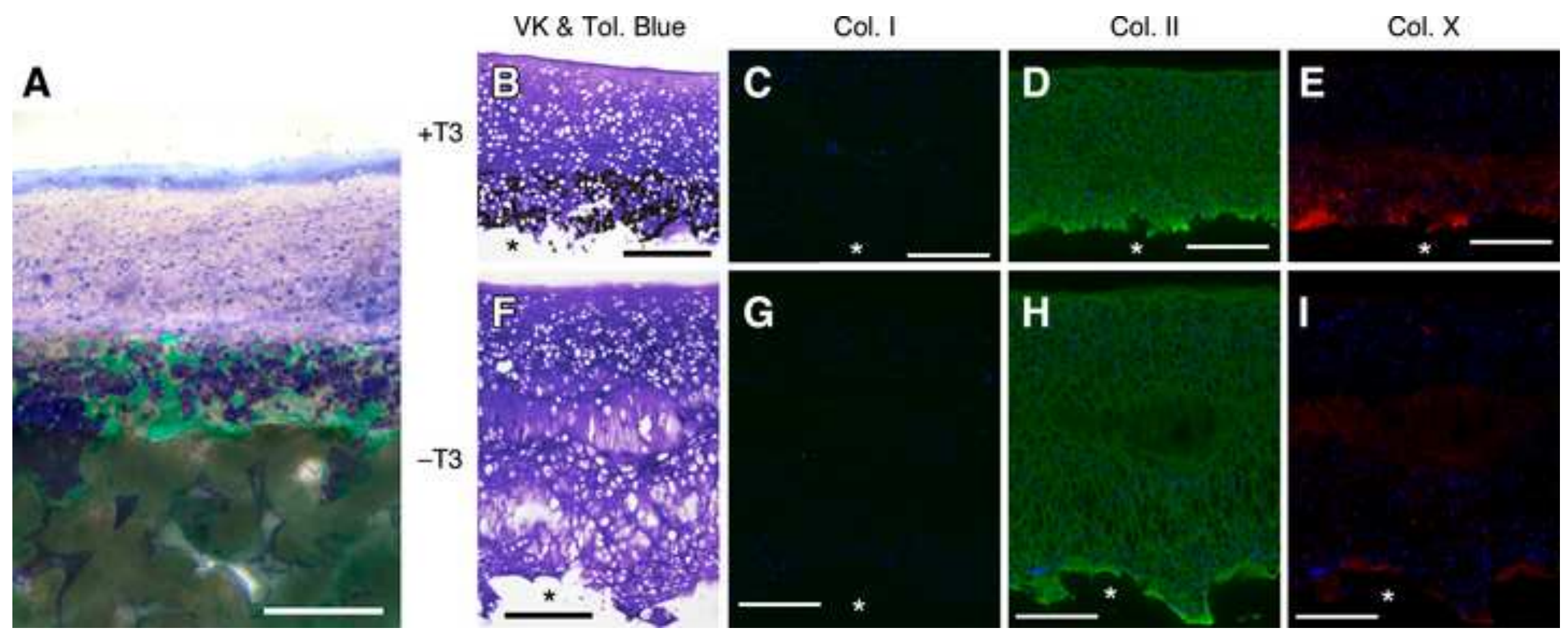

Click here to download high resolution image 

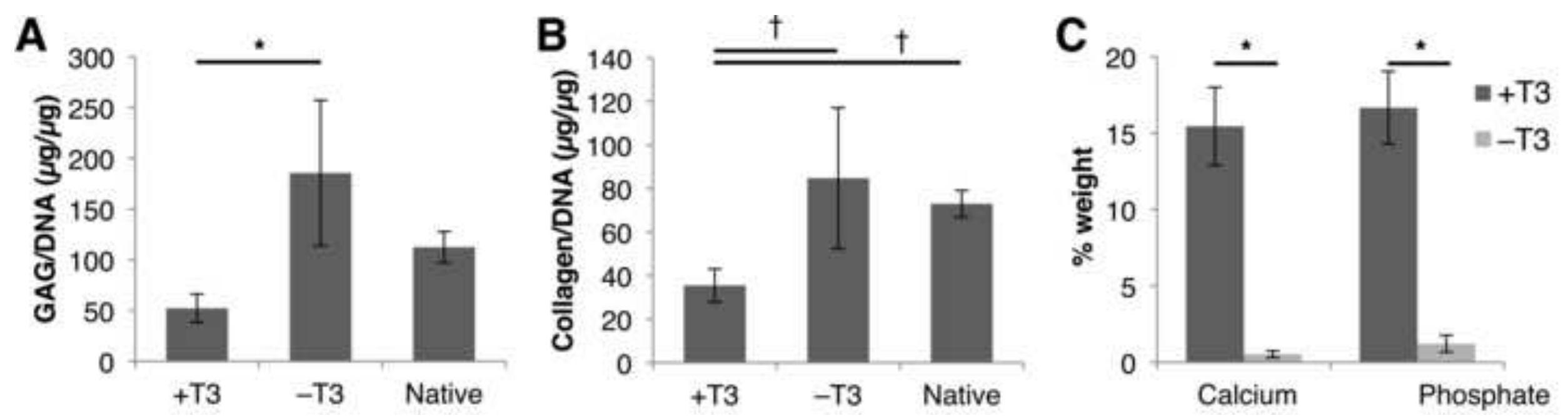

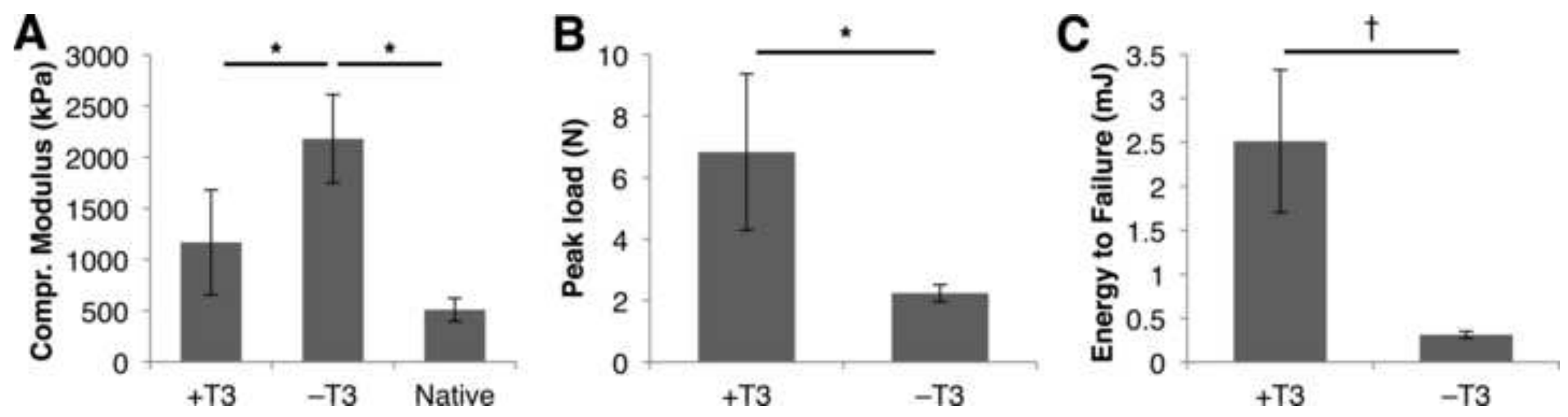


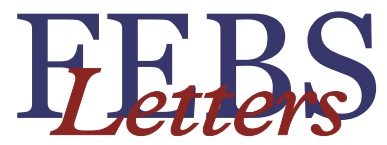

\title{
Expression of type I collagen and tenascin $\mathrm{C}$ is regulated by actin polymerization through MRTF in dedifferentiated chondrocytes
}

\author{
Justin Parreno $^{\text {a,b,d }}$, Sneha Raju ${ }^{\text {a,b }}$, Mortah Nabavi Niaki ${ }^{\text {a,b,e }}$, Katarina Andrejevic a,b,d , Amy Jiang a,b, \\ Elizabeth Delve ${ }^{\mathrm{a}, \mathrm{b}, \mathrm{e}}$, Rita Kandel ${ }^{\mathrm{a}, \mathrm{b}, \mathrm{c}, \mathrm{d}, \mathrm{e}, *}$ \\ ${ }^{a}$ CIHR-BioEngineering of Skeletal Tissues Team, Mount Sinai Hospital, University of Toronto, Toronto, Canada \\ ${ }^{\mathrm{b}}$ Lunenfeld-Tanenbaum Research Institute, Mount Sinai Hospital, Toronto, Canada \\ ${ }^{\mathrm{c}}$ Department of Laboratory Medicine and Pathobiology, University of Toronto, Canada \\ ${ }^{\mathrm{d}}$ Department of Pathology and Laboratory Medicine, Mount Sinai Hospital, Toronto, Canada \\ ${ }^{\mathrm{e}}$ Institute of Biomaterials and Biomedical Engineering, University of Toronto, Canada
}

\section{A R T I C L E I N F O}

\section{Article history:}

Received 10 June 2014

Revised 5 August 2014

Accepted 11 August 2014

Available online 19 August 2014

Edited by Lukas Huber

\section{Keywords:}

Chondrocyte

Dedifferentiation

Actin

Myocardin related transcription factor

Tenascin C

Collagen

\begin{abstract}
A B S T R A C T
This study examined actin regulation of fibroblast matrix genes in dedifferentiated chondrocytes. We demonstrated that dedifferentiated chondrocytes exhibit increased actin polymerization, nuclear localization of myocardin related transcription factor (MRTF), increased type I collagen (col1) and tenascin C (Tnc) gene expression, and decreased Sox9 gene expression. Induction of actin depolymerization by latrunculin treatment or cell rounding, reduced MRTF nuclear localization, repressed col1 and Tnc expression, and increased Sox9 gene expression in dedifferentiated chondrocytes. Treatment of passaged chondrocytes with MRTF inhibitor repressed col1 and Tnc expression, but did not affect Sox9 expression. Our results show that actin polymerization regulates fibroblast matrix gene expression through MRTF in passaged chondrocytes.
\end{abstract}

() 2014 Federation of European Biochemical Societies. Published by Elsevier B.V. All rights reserved.

\section{Introduction}

Autologous cell-based articular cartilage repair requires access to a source of sufficient cells capable of producing articular cartilage matrix. Expansion of chondrocytes in two-dimensional (2D) polystyrene cell culture is capable of providing a large number of cells, however, this results in dedifferentiation. Dedifferentiation is characterized by reduced expression of articular cartilage matrix molecules such as aggrecan (acan) and type II collagen (col2) [1-3] as well as increased expression of fibroblastic matrix molecules such as type I collagen (col1) and tenascin C (tnc) [3,4]. This is characteristic of fibrocartilage-type matrix which is biomechanically inferior and incapable of withstanding the loading associated with daily use (fails after 7 years) [5].

In addition, passaged cells are large, spread, and have actin organized into stress fibers. This is in contrast to small, round primary chondrocytes with cortical distribution of actin. Cell shape and actin stress fiber organization are important in the regulation

* Corresponding author. Address: Department of Pathology and Laboratory Medicine, Mount Sinai Hospital, 600 University Avenue, Toronto M5G 1X5, Canada.

E-mail address: rkandel@mtsinai.on.ca (R. Kandel). of the dedifferentiated cell phenotype $[1,3,6]$. Exposure of passaged chondrocytes to actin depolymerization agents to prevent cell spreading or induce cell rounding not only leads to reexpression of cartilage matrix genes [7], but also reduced col1 expression [8].

Sox9 is known as the master transcriptional factor regulating expression of chondrogenic matrix molecules [9], such as col2 and acan. For chondrocytes, actin depolymerization has been shown to regulate sox9 activity through protein kinase A (PKA) signaling [10]. While the actin based pathways that enhance cartilage matrix genes has been elucidated, the pathway(s) regulating fibroblast matrix genes, such as col1 and tnc in dedifferentiated cells remains unclear. In other cell types, actin has been shown to regulate col1 and tnc gene expression through the myocardin related transcription factor (MRTF) [11,12]. MRTF is a co-activator of serum response factor (SRF). The MRTF/SRF complex binds to the $\mathrm{CArG}$ regulatory sequence $\left(\mathrm{CC}(\mathrm{A} / \mathrm{T})_{6} \mathrm{GG}\right)$ in the promoter regions of target genes. In addition, MRTF also contains actin binding motifs and has strong affinity for the monomeric, globular (g-) actin [13]. Depolymerization of filamentous (f-) actin into g-actin results in MRTF binding to g-actin and localizing MRTF in the cytoplasm resulting in a decrease in MRTF regulated gene expression. 
The signaling mechanisms regulating fibroblastic gene expression in dedifferentiated chondrocytes is important if passaged chondrocytes are to be used for cartilage repair. To our knowledge, no studies have identified MRTF regulation of gene expression in passaged chondrocytes. The hypothesis being examined in this study is that chondrocyte passaging on $2 D$ polystyrene promotes cell spreading and actin polymerization resulting in an increased proportion of MRTF in the nucleus and enhanced expression of fibroblast matrix genes, col1 and tnc. Preventing or limiting nuclear translocation of MRTF either through MRTF chemical inhibition or through actin depolymerization will repress fibroblast matrix gene expression.

\section{Materials and methods}

\subsection{Cell culture}

Bovine articular chondrocytes were isolated and passaged twice (P2) as described previously with slight modifications [14]. Approximately $1.5 \times 10^{3}$ cells $/ \mathrm{cm}^{2}$ were initially seeded and maintained in complete media (Ham's F12 supplemented with 5\%FBS and anti-mycotic/-biotic). After approximately 13-15 days in culture when cells reached $80-90 \%$ confluency, the cells were trypsinized and were considered as passaged once (P1) chondrocytes. P1 chondrocytes were then re-seeded at $1.5 \times 10^{3}$ cells $/ \mathrm{cm}^{2}$ and cultured for another 7-9 days until $80-90 \%$ confluency was reached. These cells were trypsinized and then seeded at $3 \times 10^{4}$ cells $/ \mathrm{cm}^{2}$ for the different experiments. To investigate f-actin and MRTF localization, cells were plated on glass slides in complete media. For gene expression studies, cells were seeded on polystyrene. After 2 days, cells were washed in PBS prior to processing. To investigate the effects of cell rounding, cells were cultured in suspension on agarose. 2\% agarose was prepared in Ham's F12 and autoclave sterilized. Molten agarose was overlaid onto culture vessels to a thickness of $\sim 2 \mathrm{~mm}$.

\subsection{Treatment of $P 2$ chondrocytes with latrunculin B or CCG1423}

P2 chondrocytes were seeded either on glass slides (for immunocytochemistry), on polystyrene 6 well plates (for RT-PCR), or on T175 polystyrene flasks (protein analysis). For latrunculin B treatment, P2 chondrocytes were seeded in complete media. After 2 days, media was replenished either with complete media containing $1 \mu \mathrm{m}$ latrunculin B or an equivalent volume of DMSO carrier control. For CCG1423 treatment, P2 chondrocytes were seeded in serum reduced media (Ham's F12 supplemented with $0.5 \%$ FBS and anti-mycotic/-biotic). After 2 days, media was replenished with fresh serum reduced media containing $10 \mu \mathrm{M}$ CCG1423 or an equivalent volume of DMSO carrier control. Following $24 \mathrm{~h}$ of treatment, cells were harvested for analysis.

\subsection{Cell area and circularity}

Cells were washed with PBS and then incubated with calcein AM (Santa Cruz) diluted in PBS (1:5000) for $10 \mathrm{~min}$ at room temperature. Images were acquired with a Leica fluorescent DM IL microscope and processed based on a previously published protocol [15]. Using Image J, images were converted to 8-bit and threshold adjusted to minimize background noise. The 'analyze particles' function was used to determine cell area and circularity. Circularity was defined as $C=4 \mathrm{pi}(A / P 2)$, where $P$ is cell perimeter and $A$ is cell area. A circularity value of 1.0 indicates perfect circular morphology whereas a value of 0.0 would indicate an elongated polygon. A total number of at least 100 cells for each condition were evaluated from at least 3 different calves.

\subsection{Immunocytochemistry}

Cells on glass slides were fixed in $4 \%$ paraformaldehyde at room temperature for $10 \mathrm{~min}$. Cells were permeabilized in $0.1 \%$ triton/ PBS for $30 \mathrm{~min}$ and then incubated with rabbit polyclonal antiMRTF-a antibody (Abcam; Cambridge, MA, USA; dilution of 1:200 in $0.1 \%$ triton/PBS). After overnight incubation, slides were washed and then incubated in anti-rabbit IgG conjugated Alexa488 (dilution $1: 250$ in $0.1 \%$ triton/PBS) followed by an hour incubation in Alexa-598 phalloidin conjugate as a counterstain. Images were obtained at a standard location in the cells using a Nikon C1si laser scanning confocal microscope equipped with NIS Elements software.

Immunocytochemistry on suspension cultures was performed in a similar manner, however, cells were pelleted at $600 \mathrm{~g}$ for $5 \mathrm{~min}$, resuspended in $4 \%$ paraformaldehyde at a density of approximately $5 \times 10^{3}$ cells $/ 0.1 \mathrm{~mL}$, placed on a glass slide and air dried at $37^{\circ} \mathrm{C}$ for $30 \mathrm{~min}$ prior to primary antibody incubation.

\subsection{Protein extraction}

Adherent cells were scraped from 2D cultures and then pelleted by centrifugation at $800 \mathrm{~g}$ for $3 \mathrm{~min}$. For suspension cultures, cells were pelleted first in media, washed in PBS and then re-pelleted. Pellets were resuspended in RIPA buffer $(50 \mathrm{mM}$ Tris $\mathrm{HCl}$, $150 \mathrm{mM} \mathrm{NaCl}, 1 \% \mathrm{NP}-40,0.5 \%$ sodium deoxycholate, $0.1 \% \mathrm{SDS}$ ) with complete mini protease inhibitor (Roche; Manheim, Germany). Debris was removed by centrifugation at $14,000 \mathrm{~g}$ at $4{ }^{\circ} \mathrm{C}$ for 30 min and total protein was quantified using bicinchoninic acid protein assay (Thermo Scientific; Waltham, MA, USA).

Nuclear and cytoplasmic protein was extracted from cells using a NE-PER Nuclear and Cytoplasmic Extraction kit (Thermo Scientific). Cells were first scraped off flasks, pelleted, and then nuclear and cytoplasmic fractions were separated as per manufacturer's instructions and then quantified.

\subsection{Analysis of g-/f-actin protein levels}

G- and f-actin portions were separated by triton solubility as previously described with slight modifications [16]. Following treatment, cells were washed in PBS, then scraped off polystyrene flasks, suspended in PBS and pelleted. Pellets were resuspended in $250 \mu \mathrm{L}$ of extraction buffer ( $0.1 \%$ triton X-100 in PBS and complete protease inhibitor) and incubated for 5 min under slight agitation. The samples were then centrifuged at $15,000 \mathrm{~g}$ at $4{ }^{\circ} \mathrm{C}$ for $5 \mathrm{~min}$. The soluble portions (supernatant; predominantly globular actin) was collected. The triton-insoluble (pellet; predominantly filamentous actin) was resuspended in $250 \mu \mathrm{L}$ of RIPA buffer and agitated for $30 \mathrm{~min}$.

\subsection{SDS-PAGE and Western blotting}

Proteins were prepared for SDS-PAGE by heating in Laemmli buffer for $10 \mathrm{~min}$ at $98^{\circ} \mathrm{C}$. To evaluate total protein extracts, $30 \mu \mathrm{g}$ of protein were separated in a $12 \%$ SDS-PAGE gel. For actin assays, equal volumes of the soluble and insoluble protein fractions were loaded. Following adequate separation, proteins were semidry transferred onto polyvinylidene fluoride (PVDF) membranes using an iBLOT transfer system (Life Technologies). Membranes were then incubated in blocking buffer (5\% skim milk) for $30 \mathrm{~min}$ and then incubated in primary antibody using the dilutions listed in Supplementary Table 1. Following an overnight incubation, membranes were washed in $0.005 \%$ Tween/PBS and then incubated at room temperature for $1 \mathrm{~h}$ in HRP conjugated secondary antibody (1:20,000; Abcam). Chemiluminescent signals were developed using Pierce ECL Western Blot Substrate (Thermo Scientific) in a 
dark room. Protein bands were semi-quantified through densitometry using Image J software.

\subsection{RNA isolation and reverse transcription-polymerase chain reaction (RT-PCR)}

Total RNA was isolated from cells using TRIzol reagent according to manufacturer's instructions. RNA was reverse transcribed with SuperScript III Reverse Transcriptase (Life Technologies, Carlsbad, CA, USA) and amplified utilizing a Mastercycler Thermocycler (Eppendorf, AG, Hamburg, Germany). Relative real-time PCR was performed using Fast SYBR Green I Master Mix (Applied Biosystems, Foster City, CA, USA) with primers designed for the genes listed in Supplementary Table 2. Thermal cycling and fluorescent detection was performed using a LightCycler 96 Real-Time PCR System (Roche).

\subsection{Statistical analysis}

Each experiment was performed with 3-6 replicates and repeated 3-4 times in independent experiments. T-tests were utilized to analyze differences between two groups. Univariate analysis of variance (ANOVA) was performed when an analysis between more than two groups was required with Tukeys post hoc to detect differences between groups.

\section{Results}

\subsection{P2 chondrocytes have reduced $g$-/f-actin and increased nuclear accumulation of MRTF}

P2 chondrocytes were significantly larger and more spread as compared to P0 chondrocytes (Fig. 1A; S1 and S2). P0 chondrocytes organized actin cortically whereas in $\mathrm{P} 2$ chondrocytes actin was organized into stress fibers (Fig. 1B). P0 chondrocytes had a significantly higher g-/f-actin as compared to P2 chondrocytes (Fig. 1C). Total MRTF levels were significantly higher in P2 chondrocytes (Fig. 1D) and there were a greater proportion of P2 chondrocytes with MRTF located in the nucleus as compared to P0 chondrocytes (91\% in $\mathrm{P} 2$ versus $67 \%$ in $\mathrm{P0} ; \mathrm{P}<0.05$; Fig. $1 \mathrm{~B}$ and $\mathrm{E}$ ).

\subsection{CCG1423 treatment resulted in cytoplasmic localization of MRTF, and decreased col1 and tnc expression}

Regulation of gene expression by MRTF was investigated by exposing P2 chondrocytes to the small molecule inhibitor,
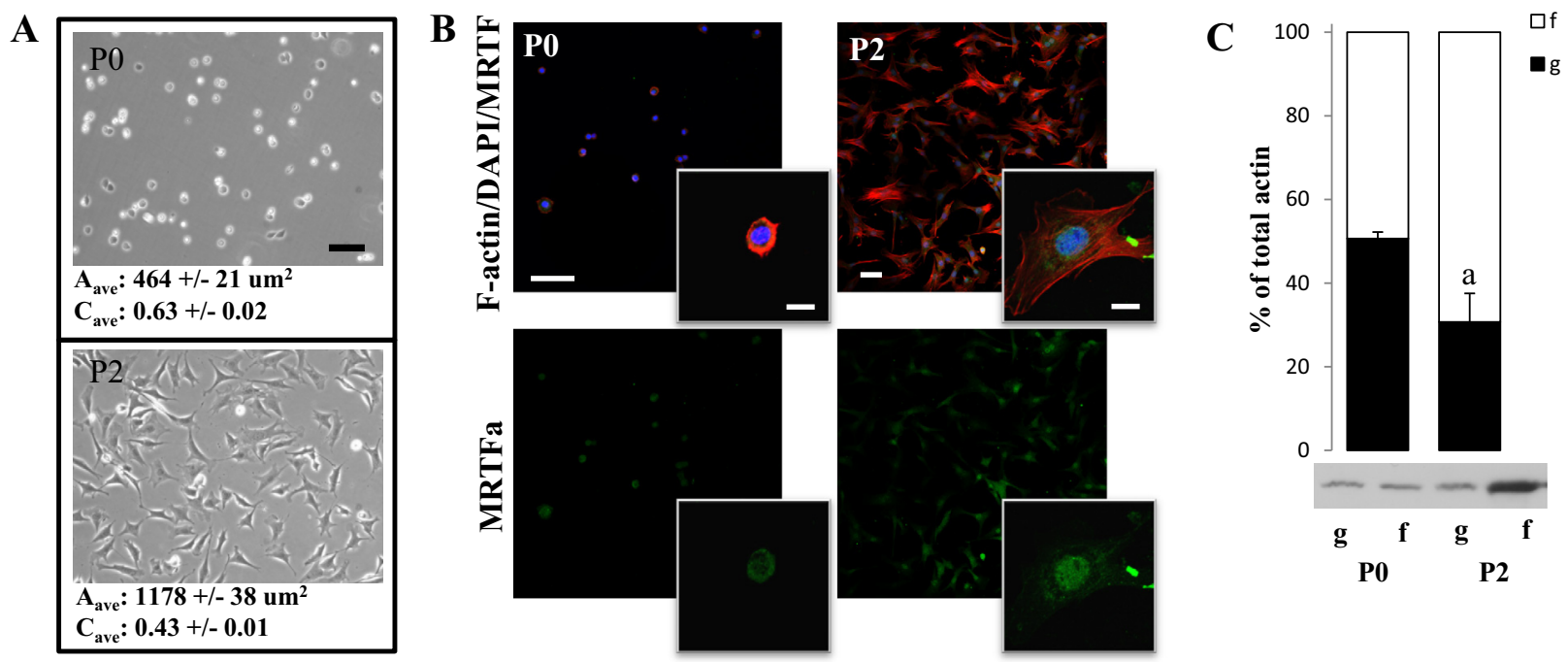

$\mathbf{D}$

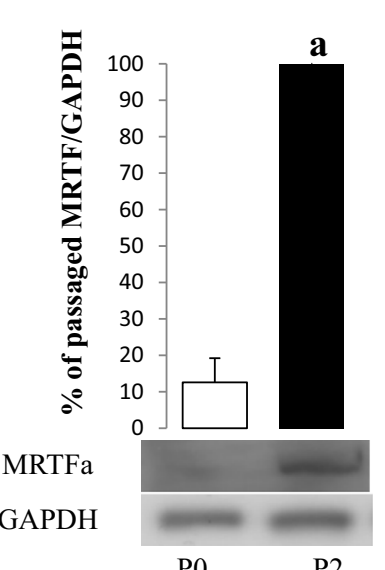

$\mathbf{E}$

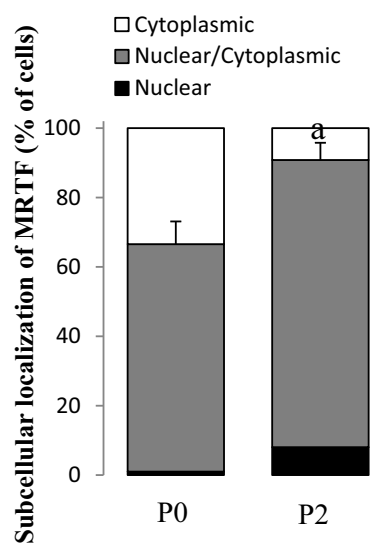

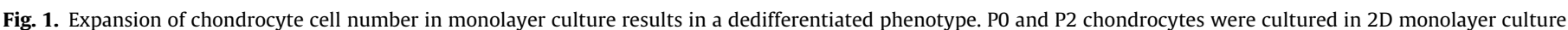

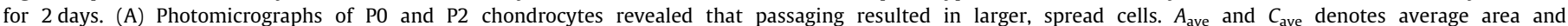

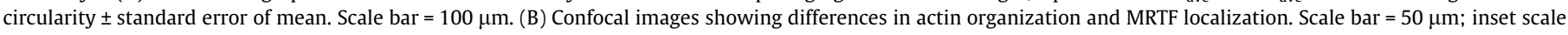

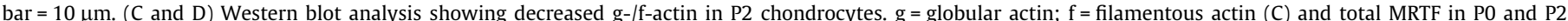

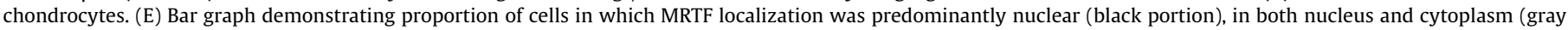
portion), or predominantly cytoplasmic (white portion). ${ }^{\mathrm{a}} \mathrm{P}<0.05$ as compared to P0 chondrocytes. 
CCG1423, which specifically represses MRTF nuclear localization [17] through binding of the N-terminal basic domain of MRTF [18]. CCG1423 experiments were conducted in serum-free medium to reduce serum activation of gene expression [19]. P2 chondrocytes treated with CCG1423 (CCG1423+) maintained a flattened, spread morphology (Fig. 2A) with actin organized into stress fibers (Fig. 2B), although the cells exposed to CCG1423 were significantly smaller (Figs. 2A and S3) and rounder compared to untreated P2 chondrocytes (Fig. S4). There was a trend toward increased g-/factin, however, this was not significantly different between treated and untreated (CCG1423-) cells (Fig. 2C). MRTF localization was significantly altered as there were a significantly lower proportion of cells that had nuclear MRTF (Fig. 2B and D).

The mRNA levels for col1 and tnc were elevated in P2 chondrocytes (Fig. 2E) 741- and 2.4-fold, respectively. CCG1423 treatment resulted in decreased col1 and tnc mRNA levels 3.4- and 1.4-fold, respectively. Nevertheless, these were still elevated over P0 chondrocyte levels. As compared to P0 chondrocytes, acan and col2 remained low in control as well as CCG1423 treated P2 chondrocytes (Fig. S5). While there was a trend toward increased sox9 following CCG1423 exposure, this was not statistically significant.
Since col1 was substantially modulated in P2 chondrocytes following CCG1423 treatment, the effect of CCG1423 on col1 protein was investigated. The antibody utilized in this study recognizes both the $\alpha 1$ and $\alpha 2$ chains of col1. Western blots using this antibody revealed higher amounts of $\alpha 1$ chain as compared to the $\alpha 2$ chain in P2 chondrocytes cultured in complete media (data not shown). However, in serum-reduced conditions the band intensity of $\alpha 2$ was greater than $\alpha 1$ (Fig. 2F) suggesting serum-dependent differential regulation of the chains. Nevertheless, CCG1423 treatment repressed both $\alpha 1$ and $\alpha 2$ chains of type I collagen in serumreduced conditions.

\subsection{Latrunculin B treatment of $P 2$ chondrocytes resulted in cell rounding, increased $g$-/f-actin, cytoplasmic localization of MRTF, reduced expression of col1 and tnc, and enhanced sox9 mRNA levels}

To investigate the effect of actin depolymerization on P2 chondrocytes, cells were exposed to latrunculin. Latrunculin promotes actin depolymerization by binding in a $1: 1$ ratio with g-actin [20]. Unlike other actin depolymerization promoting agents, such as cytochalasin $\mathrm{D}$, latrunculin does not interfere with the
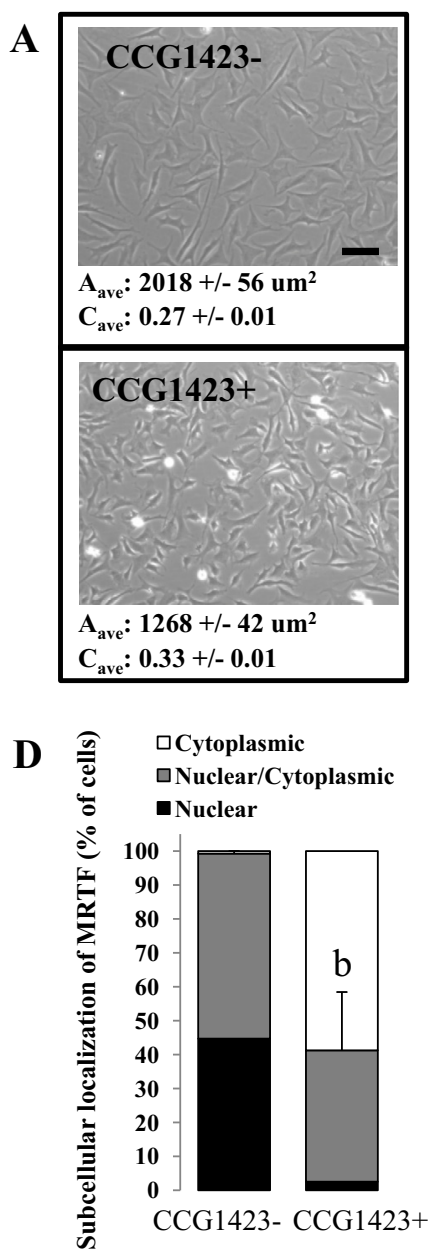
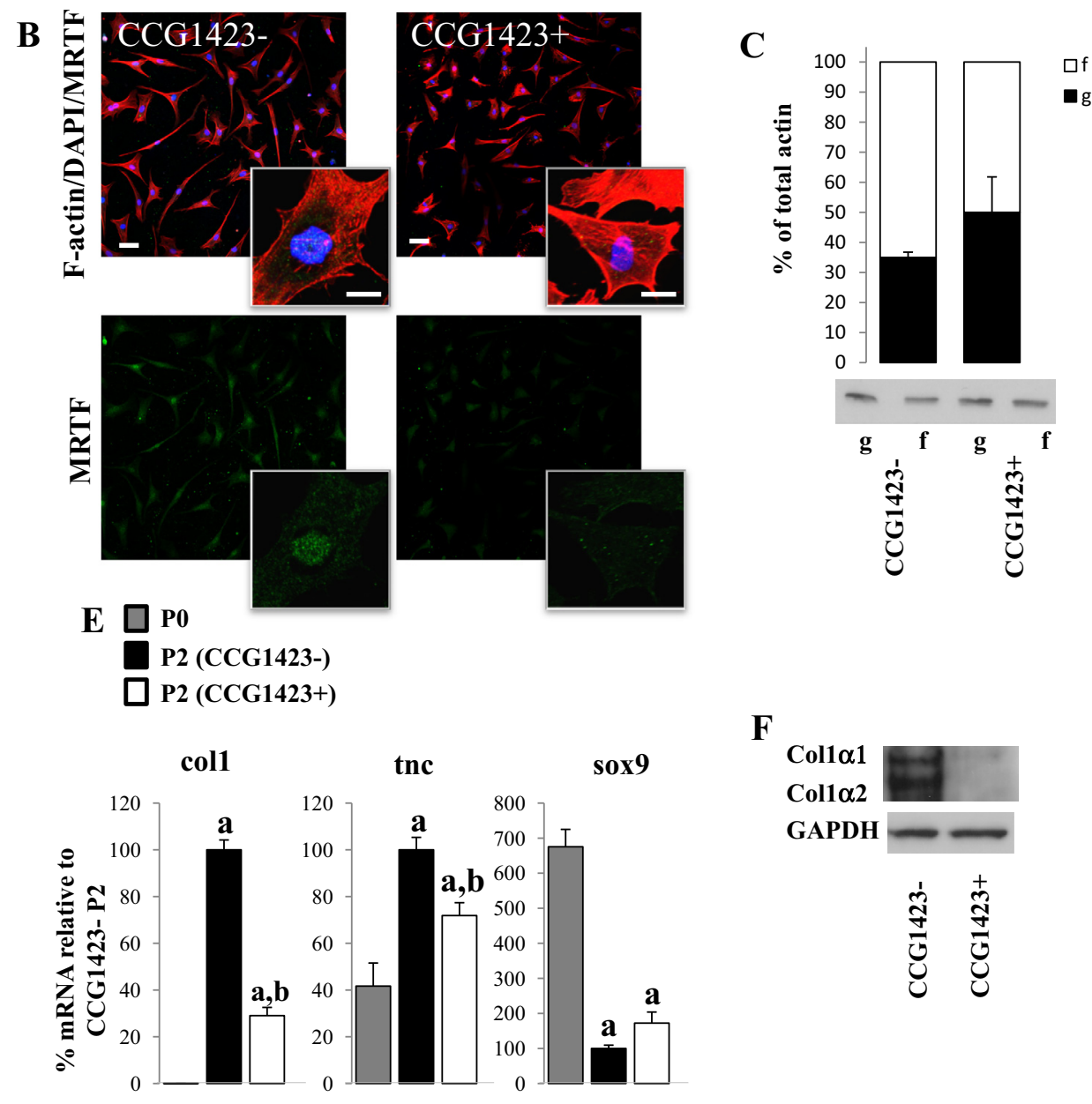

F

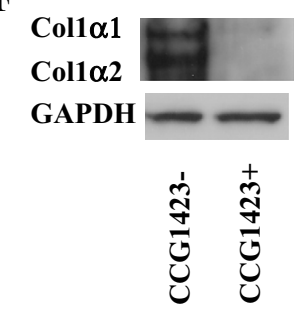

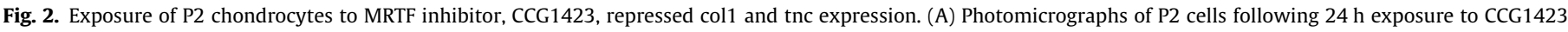

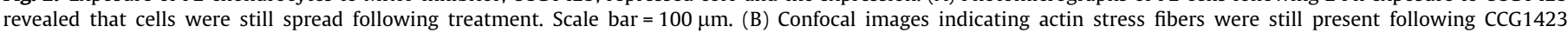

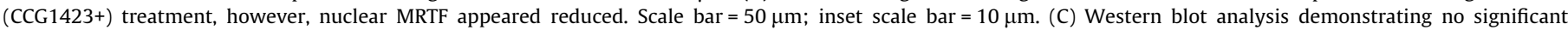

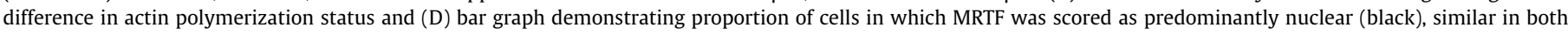

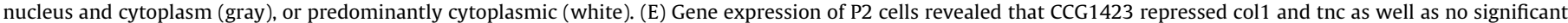

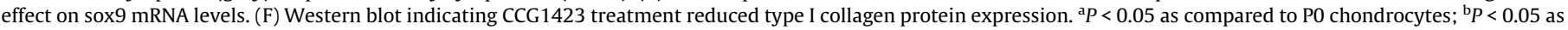
compared to P2 control cells. 
interaction between MRTF and actin [21] and was therefore utilized in this study. Exposure of P2 chondrocytes to latrunculin resulted in significantly smaller cells (Figs. $3 \mathrm{~A}$ and S6) that were rounder (Fig. S7) and had diminished actin stress fiber organization (Fig. 3B). Latrunculin treated P2 chondrocyte actin organization did not fully resemble P0 chondrocytes as cortical actin distribution was not established. However, compared to untreated cells the g-/f-actin ratio was significantly enhanced by latrunculin treatment (Fig. 3C).

Latrunculin treatment resulted in a smaller proportion of P2 chondrocytes that had nuclear MRTF as compared to control cells (9\% in latrunculin+ versus $80 \%$ in latrunculin-; $P<0.05$; Fig. $2 \mathrm{~B}$ and $\mathrm{D})$. This was confirmed by Western blot analysis, which showed a reduced amount of nuclear MRTF in latrunculin treated cells as compared to untreated control cells (Fig. 3E).

Exposure of P2 cells to latrunculin repressed col1 and tnc mRNA levels 2.0- and 1.5-fold respectively (Fig. 3F). Despite this reduction, col1 and tnc mRNA levels in P0 chondrocytes were still significantly lower than latrunculin treated P2 chondrocytes. Latrunculin treatment did not lead to recovery of col2 or acan expression (Fig. S8). However, it led to a 5.1-fold recovery in sox9 mRNA expression which was still significantly lower than P0 chondrocyte levels (Fig. 3F).

\subsection{Cell rounding through culture of P2 chondrocytes in suspension} resulted in increased $g$-/f-actin, cytoplasmic localization of MRTF, and reduced expression of col1 and thc

Cells in suspension culture were significantly smaller (Figs. 4A and S9) and rounder (Fig. S10) as compared to cells in monolayer. Cells also had reduced f-actin with no apparent stress fibers (Fig. 4B). Furthermore, cells in suspension had significantly elevated g-/f-actin ratio (Fig. 4C) and a smaller proportion of cells with nuclear MRTF as compared to cells in monolayer culture (Fig. 4D).

Suspension culture significantly repressed gene expression levels for col1 and tnc 9.8-fold and 1.6-fold, respectively. Despite the reduction in gene expression, the levels in P0 chondrocytes were still significantly lower as compared to those in P2 chondrocytes in suspension. Col2 and acan were not recovered by culture in suspension (Fig. S10). While sox9 mRNA levels were increased 5.9fold, sox9 levels for cells in suspension were still significantly lower than P0 chondrocytes.
A

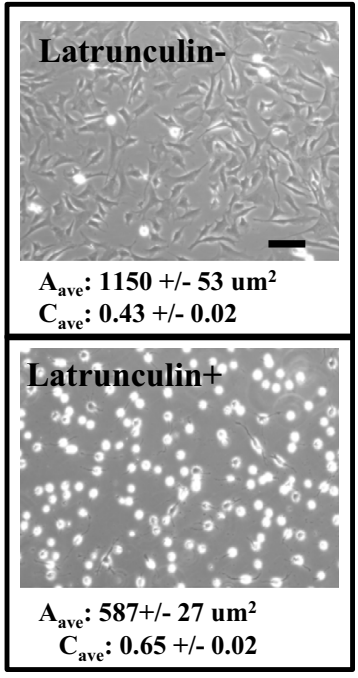

D

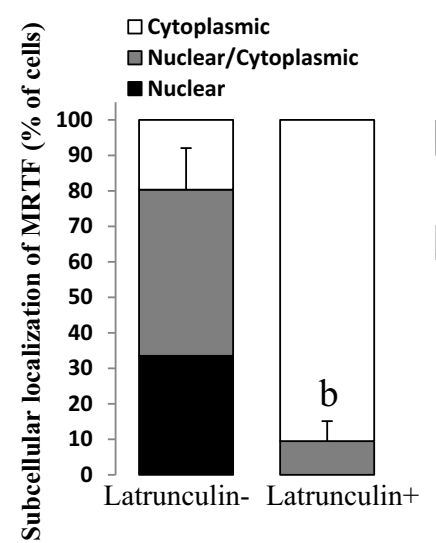

B

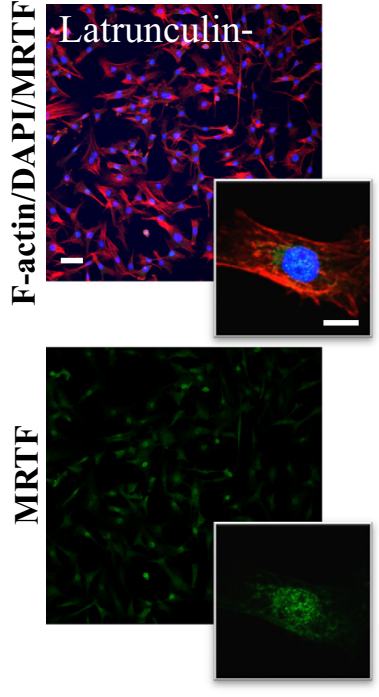

E

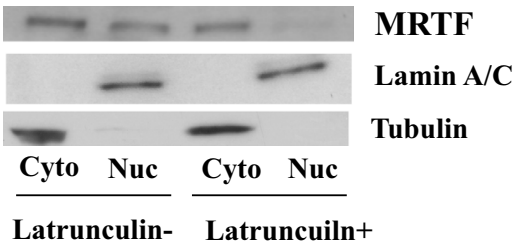

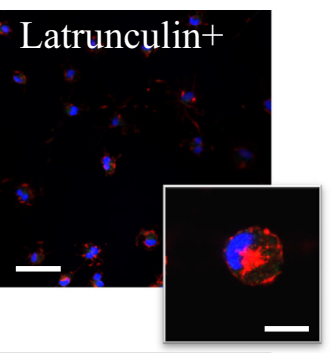

C

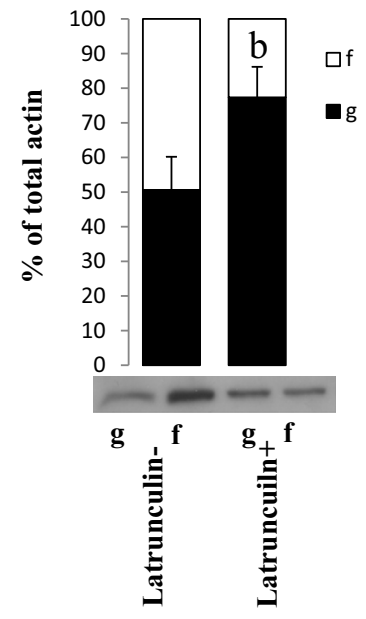

F $\square$ Po

P2 (Latrunculin-)

P2 (Latrunculin+)

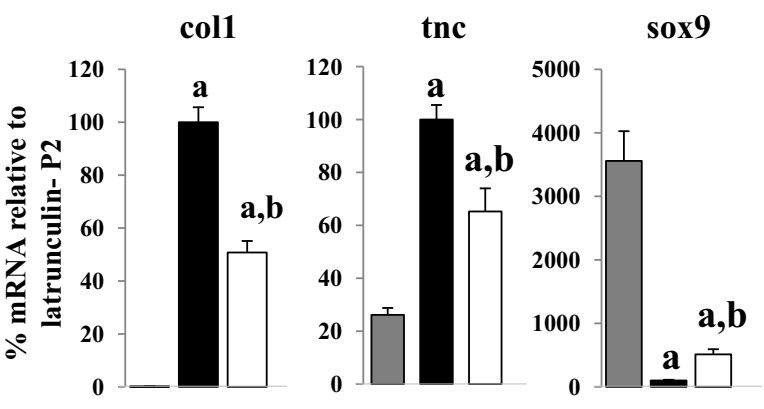

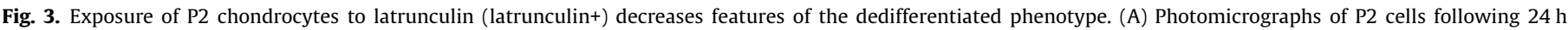

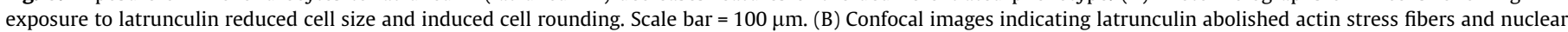

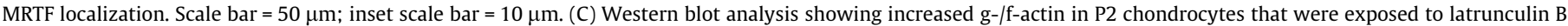

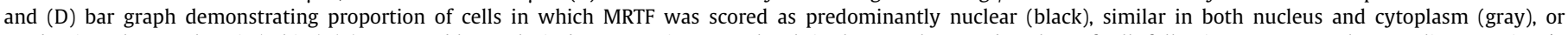

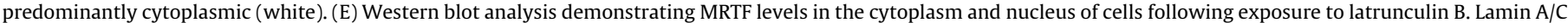

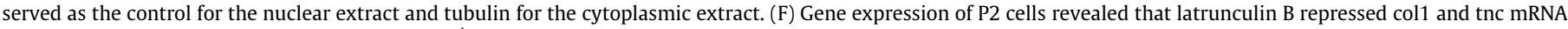
levels. ${ }^{\mathrm{a}} \mathrm{P}<0.05$ as compared to P0 chondrocytes; ${ }^{\mathrm{b}} \mathrm{P}<0.05$ as compared to P2 control cells. 

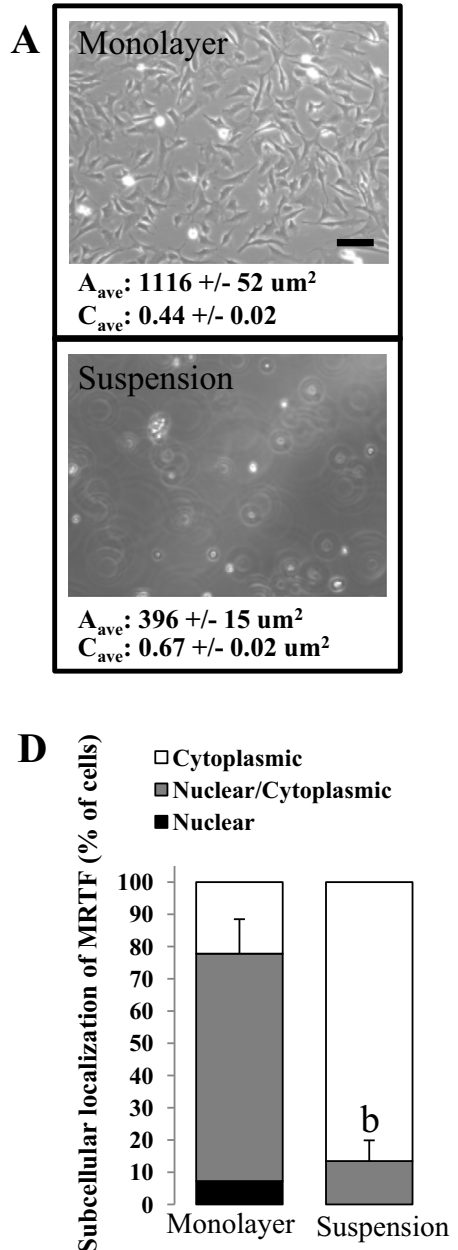
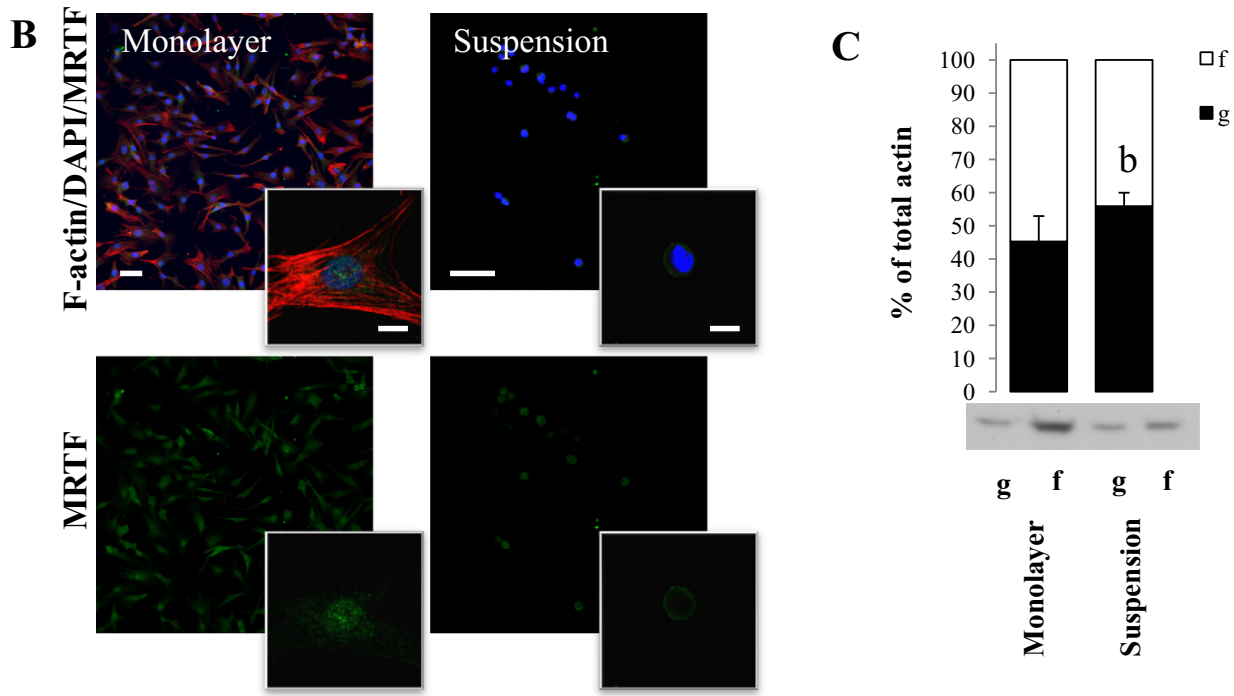

$\mathrm{E} \square \mathrm{P}$ (Monolayer)
P2 (Monolayer)
$\square \mathrm{P} 2$ (Suspension)

col1

tnc

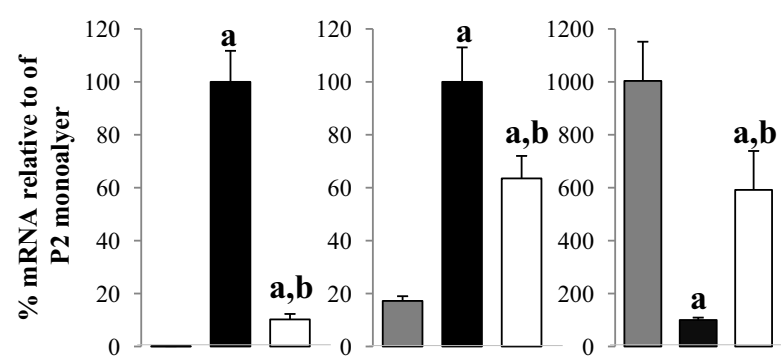

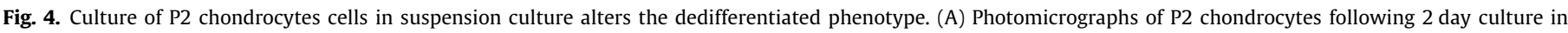

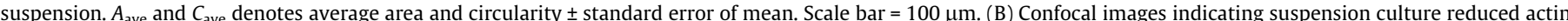

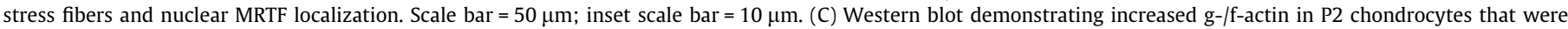

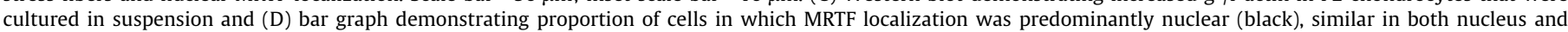

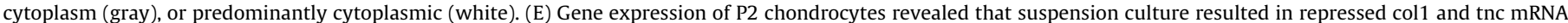
levels. ${ }^{\text {a }} P<0.05$ as compared to P0 chondrocytes; ${ }^{b} P<0.05$ as compared to P2 control cells.

\section{Discussion}

Monolayer expansion of chondrocytes for cell-based articular cartilage repair is problematic as it results in dedifferentiated chondrocytes that produce fibrocartilage-type matrix. This study demonstrated that the expression of fibroblast matrix genes, col1 and tnc, is regulated by actin polymerization through MRTF in dedifferentiated chondrocytes. These cells have increased actin polymerization and a higher proportion of MRTF nuclear localization as compared to primary chondrocytes. Additionally, inducing actin depolymerization in dedifferentiated chondrocytes resulted in cytoplasmic localization of MRTF and reduced col1 and tnc gene expression.

Dedifferentiated cells show changes in cell shape and actin organization, both of which have been shown to be regulators of chondrogenic gene expression [6,22]. We found that fibroblastic matrix expression of col1 (similar to previous studies [8,22]) and also tnc correlated with both shape and actin organization. To further elucidate how shape or actin organization regulates fibroblastic matrix gene expression in chondrocytes, we investigated the actin polymerization status of cells as it has been shown to be a key regulator of gene expression for other cell types $[21,23]$. Firstly, we found that cell shape and actin polymerization were interrelated (Fig. 5); inducing cell rounding led to actin depolymerization and vice versa. Secondly, we observed a correlation between actin organization and polymerization status; P2 chondrocytes displaying stress fibers had a greater proportion of polymerized actin as compared to P0 chondrocytes where actin was cortically distributed. Thus the regulation of gene expression by shape or actin organization can occur through modulation of actin polymerization status. Increasing evidence has shown that the

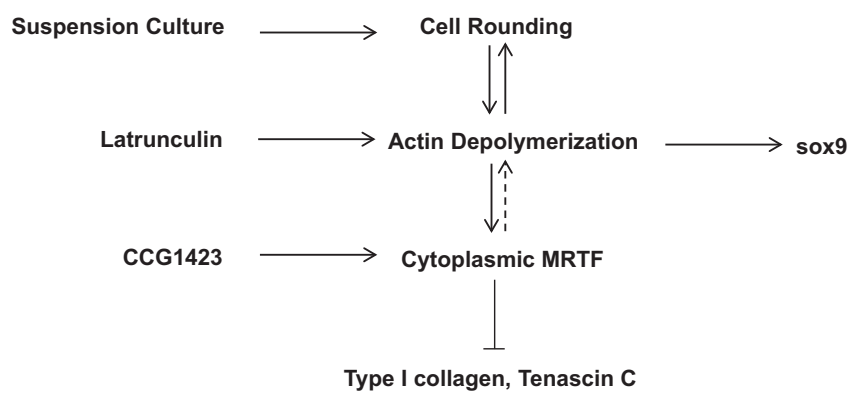

Fig. 5. Schematic of potential actin based MRTF mechanism following dedifferentiation of cells. Cell shape and actin polymerization have a bidirectional relationship and are upstream of MRTF. 
actin polymerization state regulates gene expression through the transport of actin binding proteins into and out of the nucleus $[21,23]$. MRTF in particular, which is one of the best characterized g-actin binding proteins, has the ability to regulate col1 [11] and tnc [12] gene expression in other cell types.

Our results revealed that col1 and tnc gene expression in P2 chondrocytes was dependent in part on MRTF. As shown in other cell types, nuclear localization of MRTF allows it to associate and act as a transcriptional co-activator to SRF on promoter regions [21], and therefore enhancing col1 and tnc gene expression (Fig. 5). Interestingly, we have found that culture of P2 chondrocytes in 3D micromass cultures results in actin depolymerization and repressed col1 and tnc gene expression (data not shown) which may be the result of decreased SRF activity that occurs in micromass culture of cells [24]. Nevertheless, tnc is also regulated by SRF independent mechanisms [12], therefore the exact downstream mechanism(s) by which MRTF regulates col1 and thc expression in chondrocytes requires further investigation. Furthermore although we conducted CCG1423 experiments in serumreduced conditions, CCG1423 treatment of cells in complete media also led to significantly reduced expression of col1 and tnc mRNA levels (data not shown). The magnitude of repression for tnc mRNA levels by CCG1423 treatment in complete media (1.5-fold) was similar to that in serum-reduced conditions. However, the effect on col1 gene expression was affected by serum as the magnitude of repression in complete media (1.4-fold) by CCG1423 was less than that in serum reduced conditions (3.5-fold). Interestingly serum can enhance SRF-regulated genes independent of MRTF [19] and therefore an elucidation of other pathways regulating SRF in chondrocytes is required. Additionally, further examination to determine what other genes are regulated by MRTF and/or SRF in chondrocytes is also critical. Of note, MRTF has been shown to regulate molecules associated with actin polymerization [19]. Moreover, SRF has been considered as the 'master transcriptional regulator of the cytoskeleton' with over 200 SRF target genes associated with actin [25]. Therefore a feedback loop whereby MRTF and/or SRF also regulate actin polymerization could exist (Fig. 5). While exposure of cells to CCG1423 for $24 \mathrm{~h}$ appeared to slightly increase g-/f-actin in the present study, these increases were not statistically significant. It remains to be determined if longer term MRTF inhibition through prolonged CCG1423 treatment or MRTF knockdown can induce actin depolymerization. This is of interest as MRTF could potentially have greater, yet indirect, effects on chondrogenic phenotype by regulating actin polymerization status.

The finding that actin depolymerization, but not MRTF inhibition, affected sox9 gene expression suggests that actin depolymerization directly regulates expression of sox 9 through a pathway independent of MRTF (Fig. 5). While we did not investigate the precise signaling mechanisms regulating sox 9 by actin depolymerization, actin depolymerization has been shown to enhance both sox 9 transcription and activity through a PKA dependent mechanism [10]. Thus it appears likely that actin depolymerization utilizes both MRTF and PKA signaling to regulate fibroblastic and chondrogenic matrix, respectively.

Despite the favorable changes induced through actin depolymerization, these were insufficient and further cellular modulation is required for full redifferentiation of passaged chondrocytes. This is based on our findings that actin depolymerization in P2 chondrocytes did not completely reduce col1 and tnc gene expression to levels seen in P0 chondrocytes. As well, unlike previous studies $[8,26]$, col2 and acan mRNA levels were not increased by actin depolymerization. One explanation for the lack of full redifferentiation is that the P2 chondrocytes utilized in this study may be more dedifferentiated. In comparison to other studies [7,27], we seeded cells at a lower density for passaging and also cultured them for a longer period of time. Therefore because the recovery of chondrogenic matrix gene expression is dependent on the dedifferentiation state of cells [28], full redifferentiation may require more time and another stimulus. More time appears to be required as Sox9, a potent activator of chondrogenic expression, was upregulated with actin depolymerization in our study and it is possible this chondrogenic gene expression was initiated but not fully manifested. Additionally, another stimulus may be essential as we were unable to induce cortical actin distribution in the cells which appear to be critical for full redifferentiation [29]. Stimuli used to obtain cortical actin in cells are three-dimensional (3D) culture [30] as well as insulin [31]. Interestingly, 4-week cultures of passaged chondrocytes in 3D maintained in serum-free media supplemented with insulin lead to redifferentiation of passaged chondrocytes and formation of hyaline cartilage [32].

In conclusion this study shows that actin polymerization status regulates passaged chondrocyte expression of fibroblastic matrix genes through MRTF. As fibrocartilage matrix formation is a consequence of the in vivo healing response to cartilage damage [33] as well as is characteristic of in vitro passaged chondrocytes used for autologous cell-based therapies [34], modulating MRTF would be a way to prevent or suppress fibrocartilage formation. Further studies are required to determine the pathways involved in full redifferentiation to generate chondrocytes for use in articular cartilage repair.

\section{Acknowledgments}

J.P. was supported by a University of Toronto Fellowship, a Canadian Arthritis Network - Ontario Graduate Studentship in Science and Technology and an NSERC - Alexander Graham Bell Canada Graduate Scholarship. This work was supported by Canadian Institutes of Health Research (CIHR) Grant MOP126111 and the United States Army Department of Defense reference W81XWH10-1-0787.

\section{Appendix A. Supplementary data}

Supplementary data associated with this article can be found, in the online version, at http://dx.doi.org/10.1016/j.febslet.2014.08. 012.

\section{References}

[1] Mallein-Gerin, F., Ruggiero, F. and Garrone, R. (1990) Proteoglycan core protein and type II collagen gene expressions are not correlated with cell shape changes during low density chondrocyte cultures. Differentiation 43, 204-211.

[2] Darling, E.M. and Athanasiou, K.A. (2005) Rapid phenotypic changes in passaged articular chondrocyte subpopulations. J. Orthop. Res. 23, 425-432, http://dx.doi.org/10.1016/j.orthres.2004.08.008.

[3] Elima, K. and Vuorio, E. (1989) Expression of mRNAs for collagens and other matrix components in dedifferentiating and redifferentiating human chondrocytes in culture. FEBS Lett. 258, 195-198.

[4] Cheng, T., Maddox, N.C., Wong, A.W., Rahnama, R. and Kuo, A.C. (2012) Comparison of gene expression patterns in articular cartilage and dedifferentiated articular chondrocytes. J. Orthop. Res. 30, 234-245, http:// dx.doi.org/10.1002/jor.21503.

[5] Kaul, G., Cucchiarini, M., Remberger, K., Kohn, D. and Madry, H. (2012) Failed cartilage repair for early osteoarthritis defects: a biochemical, histological and immunohistochemical analysis of the repair tissue after treatment with marrow-stimulation techniques. Knee Surg. Sports Traumatol. Arthrosc. 20, 2315-2324, http://dx.doi.org/10.1007/s00167-011-1853-x.

[6] Watt, F.M. and Dudhia, J. (1988) Prolonged expression of differentiated phenotype by chondrocytes cultured at low density on a composite substrate of collagen and agarose that restricts cell spreading. Differentiation 38, 140-147.

[7] Newman, P. and Watt, F.M. (1988) Influence of cytochalasin D-induced changes in cell shape on proteoglycan synthesis by cultured articular chondrocytes. Exp. Cell Res. 178, 199-210.

[8] Hoshiba, T., Yamada, T., Lu, H., Kawazoe, N., Tateishi, T. and Chen, G. (2008) Nuclear deformation and expression change of cartilaginous genes during in vitro expansion of chondrocytes. Biochem. Biophys. Res. Commun. 374, 688-692, http://dx.doi.org/10.1016/j.bbrc.2008.07.112.

[9] Lefebvre, V., Huang, W., Harley, V.R., Goodfellow, P.N. and de Crombrugghe, B. (1997) SOX9 is a potent activator of the chondrocyte-specific enhancer of the pro alpha1(II) collagen gene. Mol. Cell. Biol. 17, 2336-2346. 
[10] Kumar, D. and Lassar, A.B. (2009) The transcriptional activity of Sox9 in chondrocytes is regulated by RhoA signaling and actin polymerization. Mol. Cell. Biol. 29, 4262-4273, http://dx.doi.org/10.1128/MCB.01779-08.

[11] Luchsinger, L.L., Patenaude, C.A., Smith, B.D. and Layne, M.D. (2011) Myocardin-related transcription factor-A complexes activate type I collagen expression in lung fibroblasts. J. Biol. Chem. 286, 44116-44125, http:// dx.doi.org/10.1074/jbc.M111.276931.

[12] Asparuhova, M.B., Ferralli, J., Chiquet, M. and Chiquet-Ehrismann, R. (2011) The transcriptional regulator megakaryoblastic leukemia-1 mediates serum response factor-independent activation of tenascin- $C$ transcription by mechanical stress. FASEB J. 25, 3477-3488, http://dx.doi.org/10.1096/fj.11187310.

[13] Guettler, S., Vartiainen, M.K., Miralles, F., Larijani, B. and Treisman, R. (2008) RPEL motifs link the serum response factor cofactor MAL but not myocardin to Rho signaling via actin binding. Mol. Cell. Biol. 28, 732-742, http://dx.doi.org/ 10.1128/MCB.01623-07.

[14] Gan, L. and Kandel, R.A. (2007) In vitro cartilage tissue formation by co-culture of primary and passaged chondrocytes. Tissue Eng. 13, 831-842, http:// dx.doi.org/10.1089/ten.2007.13.ft-358.

[15] Fardin, M.A., Rossier, O.M., Rangamani, P., Avigan, P.D., Gauthier, N.C., Vonnegut, W., Mathur, A., Hone, J., Iyengar, R. and Sheetz, M.P. (2010) Cell spreading as a hydrodynamic process. Soft Matter 6, 4788-4799, http:// dx.doi.org/10.1039/c0sm00252.

[16] Papakonstanti, E.A. and Stournaras, C. (2002) Association of PI-3 kinase with PAK1 leads to actin phosphorylation and cytoskeletal reorganization. Mol. Biol. Cell 13, 2946-2962, http://dx.doi.org/10.1091/mbc.02-01-0599.

[17] Jin, W., Goldfine, A.B., Boes, T., Henry, R.R., Ciaraldi, T.P., Kim, E.Y., Emecan, M., Fitzpatrick, C., Sen, A., Shah, A., et al. (2011) Increased SRF transcriptional activity in human and mouse skeletal muscle is a signature of insulin resistance. J. Clin. Invest. 121, 918-929, http://dx.doi.org/10.1172/JCI41940.

[18] Hayashi, K., Watanabe, B., Nakagawa, Y., Minami, S. and Morita, T. (2014) RPEL proteins are the molecular targets for CCG-1423, an inhibitor of Rho signaling. PLoS One 9, e89016, http://dx.doi.org/10.1371/journal.pone.0089016.

[19] Esnault, C., Stewart, A., Gualdrini, F., East, P., Horswell, S., Matthews, N. and Treisman, R. (2014) Rho-actin signaling to the MRTF coactivators dominates the immediate transcriptional response to serum in fibroblasts. Genes Dev. 28 943-958, http://dx.doi.org/10.1101/gad.239327.114.

[20] Spector, I., Shochet, N.R., Blasberger, D. and Kashman, Y. (1989) Latrunculins novel marine macrolides that disrupt microfilament organization and affect cell growth: I. Comparison with cytochalasin D. Cell Motil. Cytoskeleton 13, 127-144, http://dx.doi.org/10.1002/cm.970130302.

[21] Miralles, F., Posern, G., Zaromytidou, A.I. and Treisman, R. (2003) Actin dynamics control SRF activity by regulation of its coactivator MAL. Cell 113, 329-342.

[22] Mallein-Gerin, F., Garrone, R. and van der Rest, M. (1991) Proteoglycan and collagen synthesis are correlated with actin organization in dedifferentiating chondrocytes. Eur. J. Cell Biol. 56, 364-373.
[23] Wada, K., Itoga, K., Okano, T., Yonemura, S. and Sasaki, H. (2011) Hippo pathway regulation by cell morphology and stress fibers. Development 138 , 3907-3914, http://dx.doi.org/10.1242/dev.070987.

[24] Seghatoleslami, M.R., Roman-Blas, J.A., Rainville, A.M., Modaressi, R., Danielson, K.G. and Tuan, R.S. (2003) Progression of chondrogenesis in C3H10T1/2 cells is associated with prolonged and tight regulation of ERK1/2. J. Cell. Biochem. 88, 1129-1144, http://dx.doi.org/10.1002/jcb. 10458.

[25] Miano, J.M., Long, X. and Fujiwara, K. (2007) Serum response factor: maste regulator of the actin cytoskeleton and contractile apparatus. Am. J. Physiol. Cell Physiol. 292, C70-C81, http://dx.doi.org/10.1152/ajpcell. 00386.2006.

[26] Benya, P.D., Brown, P.D. and Padilla, S.R. (1988) Microfilament modification by dihydrocytochalasin B causes retinoic acid-modulated chondrocytes to reexpress the differentiated collagen phenotype without a change in shape. J. Cell Biol. 106, 161-170.

[27] Benya, P.D. (1988) Modulation and reexpression of the chondrocyte phenotype; mediation by cell shape and microfilament modification. Pathol. Immunopathol. Res. 7, 51-54.

[28] Schulze-Tanzil, G., de Souza, P., Villegas Castrejon, H., John, T., Merker, H.J. Scheid, A. and Shakibaei, M. (2002) Redifferentiation of dedifferentiated human chondrocytes in high-density cultures. Cell Tissue Res. 308, 371-379, http://dx.doi.org/10.1007/s00441-002-0562-7.

[29] Park, E.H., Kang, S.S., Lee, Y.S., Kim, S.J., Jin, E.J., Tak, E.N. and Sonn, J.K. (2008) Integrity of the cortical actin ring is required for activation of the PI3K/Akt and p38 MAPK signaling pathways in redifferentiation of chondrocytes on chitosan. Cell Biol. Int. 32, 1272-1278, http://dx.doi.org/10.1016/j.cellbi. 2008.07.013.

[30] Haudenschild, D.R., Chen, J., Steklov, N., Lotz, M.K. and D'Lima, D.D. (2009) Characterization of the chondrocyte actin cytoskeleton in living threedimensional culture: response to anabolic and catabolic stimuli. Mol. Cell. Biomech. 6, 135-144.

31] Wang, H., Wang, A.X. and Barrett, EJ. (2012) Insulin-induced endothelial cell cortical actin filament remodeling: a requirement for trans-endothelial insulin transport. Mol. Endocrinol. 26, 1327-1338, http://dx.doi.org/10.1210/me 2012-1003.

[32] Ahmed, N., Iu, J., Brown, C.E., Taylor, D.W. and Kandel, R.A. (2014) Serum- and growth-factor-free three-dimensional culture system supports cartilage tissue formation by promoting collagen synthesis via Sox9-Col2a1 interaction. Tissue Eng. A, http://dx.doi.org/10.1089/ten.TEA.2013.0559.

[33] Metsaranta, M., Kujala, U.M., Pelliniemi, L., Osterman, H., Aho, H. and Vuorio, E. (1996) Evidence for insufficient chondrocytic differentiation during repair of full-thickness defects of articular cartilage. Matrix Biol. 15, 39-47.

[34] Roberts, S., McCall, I.W., Darby, A.J., Menage, J., Evans, H., Harrison, P.E. and Richardson, J.B. (2003) Autologous chondrocyte implantation for cartilage repair: monitoring its success by magnetic resonance imaging and histology. Arthritis Res. Ther. 5, R60-R73. 


\title{
Serum- and Growth-Factor-Free Three-Dimensional Culture System Supports Cartilage Tissue Formation by Promoting Collagen Synthesis via Sox9-Col2a1 Interaction
}

\author{
Nazish Ahmed, PhD, ${ }^{1,2}$ Jonathan Iu, BSc, ${ }^{1,2}$ Chelsea E. Brown, BSc, ${ }^{1,3}$ \\ Drew Wesley Taylor, MSc, ${ }^{1,4}$ and Rita A. Kandel, MD ${ }^{1,5}$
}

Objective: One of the factors preventing clinical application of regenerative medicine to degenerative cartilage diseases is a suitable source of cells. Chondrocytes, the only cell type of cartilage, grown in vitro under culture conditions to expand cell numbers lose their phenotype along with the ability to generate hyaline cartilaginous tissue. In this study we determine that a serum- and growth-factor-free three-dimensional (3D) culture system restores the ability of the passaged chondrocytes to form cartilage tissue in vitro, a process that involves sox9. Methods: Bovine articular chondrocytes were passaged twice to allow for cell number expansion (P2) and cultured at high density on 3D collagen-type-II-coated membranes in high glucose content media supplemented with insulin and dexamethasone (SF3D). The cells were characterized after monolayer expansion and following 3D culture by flow cytometry, gene expression, and histology. The early changes in signaling transduction pathways during redifferentiation were characterized.

Results: The P2 cells showed a progenitor-like antigen profile of $99 \% \mathrm{CD}_{4} 4^{+}$and $40 \% \mathrm{CD} 105^{+}$and a gene expression profile suggestive of interzone cells. P2 in SF3D expressed chondrogenic genes and accumulated extracellular matrix. Downregulating insulin receptor (IR) with HNMPA-(AM3) or the PI-3/AKT kinase pathway (activated by insulin treatment) with Wortmannin inhibited collagen synthesis. HNMPA-(AM3) reduced expression of Col2, Coll1, and $I R$ genes as well as Sox6 and -9. Co-immunoprecipitation and chromatin immunoprecipitation analyses of HNMPA-(AM3)-treated cells showed binding of the coactivators Sox6 and Med12 with Sox9 but reduced Sox9-Col2al binding.

Conclusions: We describe a novel culture method that allows for increase in the number of chondrocytes and promotes hyaline-like cartilage tissue formation in part by insulin-mediated Sox9-Col2al binding. The suitability of the tissue generated via this approach for use in joint repair needs to be examined in vivo.

\section{Introduction}

D EVELOPMENT OF NEW BIOLOGICAL treatments for cartilage degradation has been hampered by lack of sufficient numbers of cells that exhibit the appropriate phenotype of articular chondrocytes. The most commonly used method to increase the number of cells is to culture them in monolayer (ML) in vitro. However, under these conditions the chondrocyte phenotype changes; cells obtain a spindled morphology and lose their chondrocytic characteristics resulting in an inability to form cartilage tissue in vitro unless manipulated in some way such as coculture with differen- tiated chondrocytes. ${ }^{1,2}$ Use of passaged chondrocytes for cartilage repair is FDA approved and has been utilized clinically for autologous chondrocyte transplant for more than 10 years, although with limited success in part because of their inability to form articular cartilage in vivo. ${ }^{3}$ Therefore, developing a method that uses passaged chondrocytes to generate hyaline cartilage without any supplementation is particularly attractive as it could shorten the time to clinical translation.

It has been suggested that, with passage, as chondrocytes lose their phenotype they acquire some characteristics of mesenchymal stromal cells (MSCs) as defined by spindled morphology; higher collagen type I gene expression; cell

\footnotetext{
${ }^{1}$ CIHR-BioEngineering of Skeletal Tissues Team, Mount Sinai Hospital, University of Toronto, Toronto, Canada.

${ }^{2}$ Lunenfeld-Tanenbaum Research Institute, Mount Sinai Hospital, Toronto, Canada.

${ }^{3}$ Arizona Health Sciences Center, The University of Arizona, Tucson, Arizona.

${ }^{4}$ Division of Orthopedics, Mount Sinai Hospital, Toronto, Canada.

${ }^{5}$ Department of Pathology and Laboratory Medicine, Mount Sinai Hospital, Toronto, Canada.
} 
surface profile of $\mathrm{CD}_{105^{+}}, \mathrm{CD} 90^{+}, \mathrm{CD} 73^{+}, \mathrm{CD} 44^{+}$, $\mathrm{CD} 45^{-}$, and $\mathrm{CD} 34^{-}$; and the ability to differentiate to tissues of mesenchymal lineage. ${ }^{4-7} \mathrm{~A}$ number of conditions have been used to redifferentiate these passaged cells to chondrocytes, such as coculture or three-dimensional (3D) culture. ${ }^{8,9}$ All of these approaches require the presence of fetal bovine serum (FBS), exogenous hormones, and/or growth factors of the transforming growth factor (TGF) $\beta$ familyconditions that can trigger hypertrophic differentiation or fibrocartilage formation. ${ }^{10,11}$ Proteomic analysis shows that FBS contains fibronectin, collagen type 2, and collagen type 1 that may promote cell attachment. It also contains growth factors, such as FGF, TGF $\beta 1$, glial growth factor, and prepro-insulin-like growth factor 1 (PIlGF-signal peptide containing IGF-1, functionally similar to insulin), in lot dependent concentrations. ${ }^{12}$ Hence, with FBS supplementation reproducibility becomes dependent on the batch of serum. The xenogeneic nature of FBS is also a concern for tissue engineering both in terms of the potential for inducing an immune response and/or transmitting disease. ${ }^{13}$ The serum substitute ITS + (insulin, transferrin, selenium, and dexamethasone; BD Bioscience) is often used to circumvent these limitations and is commonly used together with TGF $\beta$ to differentiate MSCs to chondrocytes. This may be due in part to the presence of insulin, a hormone highly conserved among vertebrates. It is a component of ITS + and is known to have effects on cell survival, proliferation, differentiation, metabolism, and during development. ${ }^{14}$ Insulin binds to the insulin receptor (IR) that activates cytoplasmic substrates insulin receptor substrate 1 (IRS-1) and/or Shc that in turn activate ERK-MAPK, PI-3 kinase, and/or Akt signal transduction pathways. ${ }^{15}$ These pathways have been implicated in regulating chondrogenesis, although Akt activation has been shown to favor chondrocyte hypertrophy. ${ }^{16}$ Importantly chondrocytes express functional IRs and levels may be altered in osteoarthritic chondrocytes. ${ }^{17}$ Recently insulin has been shown to regulate Sox9 expression in mesenchymal cells. ${ }^{18}$ Sox 9 , the master transcription factor regulating chondrogenesis, is expressed at the earliest stage of cartilage anlagen formation by chondrocytes. ${ }^{19}$ Sox 9 requires additional coactivators, such as Sox5, Sox6, Med12, and SP1, to form a functional transcriptional complex ${ }^{20,21}$ that regulates gene expression of many cartilage-related molecules, including Col2a1, Col9a2, Coll1a2, aggrecan, and cartilage oligomeric matrix protein $(C O M P) .^{20,22-24}$

This study demonstrates that in the presence of media supplemented with ITS + instead of FBS, passaged bovine chondrocytes when grown on collagen-type-II-coated membrane inserts revert back to articular chondrocytes that form hyaline cartilage tissue. The insulin in the culture media contributes to the ability of the redifferentiating cells to form cartilage tissue by sox9-mediated collagen II gene and protein expression. Understanding the mechanism(s) regulating redifferentiation will allow identification of the conditions that will support this redifferentiation process in human chondrocytes.

\section{Materials and Methods}

\section{Cell isolation and culture}

Bovine articular chondrocytes (BACs) were harvested by enzymatic digestion from cartilage obtained from bovine metacarpo-phalangeal joints (6-9 months old) as described previously. ${ }^{19}$ BACs $\left(2000\right.$ cells $\left./ \mathrm{cm}^{2}\right)$ were cultured in ML in $5 \%$ FBS and passaged twice (P2) to attain up to 200-fold increase in cell number. P2 $\left(1.5 \times 10^{6}\right.$ cells) were seeded onto type-II-collagen-coated Millicell ${ }^{\circledR}$ culture inserts $\left(60 \mathrm{~mm}^{2}\right.$; Millipore) and cultured for up to 4 weeks in serum-free 3D culture (SF3D) consisting of high glucose content medium (HG) Dulbecco's modified Eagle's medium (DMEM; $4.5 \mathrm{~g} /$ L), ITS + $(10 \mu \mathrm{g} / \mathrm{mL}$ insulin, $0.5 \mu \mathrm{g} / \mathrm{mL}$ transferrin, $0.67 \mathrm{ng} /$ $\mathrm{mL}$ selenium, $5.35 \mu \mathrm{g} / \mathrm{mL}$ linoleic acid, and $1.25 \mathrm{mg} / \mathrm{mL}$ BSA; BD Bioscience, MA), proline $(40 \mu \mathrm{g} / \mathrm{mL})$, pyruvate $(110 \mu \mathrm{g} / \mathrm{mL})$, dexamethasone $(0.1 \mu \mathrm{M})$, and ascorbate-2$\mathrm{PO}_{4}(50 \mu \mathrm{g} / \mathrm{mL})$. As controls, cells were grown in serumcontaining 3D culture (SC3D) with $20 \%$ FBS.

\section{Inhibition studies}

Twenty-four hours after seeding, the P2 cells were serum or ITS + starved for $18 \mathrm{~h}$ and treated with the IR inhibitor HNMPA-(AM3) $(100 \mu \mathrm{M} \text {; Enzo Life Sciences) })^{25}$ and PI-3 kinase (upstream of AKT) inhibitors Wortmannin $(5 \mathrm{ng} / \mathrm{mL}$; Sigma-Aldrich) or LY294002 (10 $\mu \mathrm{M}$; Millipore). Insulin was added after $2 \mathrm{~h}$ and tissue was harvested after $24 \mathrm{~h}$. Controls were cultures that received carrier (DMSO) only.

\section{Flow cytometry}

Cells were harvested using trypsin $(1 \times$ for $5 \mathrm{~min})$, allowed to recover for $30 \mathrm{~min}$ in $2 \%$ FBS containing phosphatebuffered saline (PBS), and stained with either CD105-PE or CD44-PE (12-1057 and 12-0441; eBioscience). Cells were analyzed by EPICS XL FACS and Kaluza analysis software (Beckman Coulter).

\section{Histology and immunohistochemistry}

Tissues were fixed in 10\% formalin and embedded in paraffin, and 5- $\mu \mathrm{m}$ sections were cut. Representative sections were stained with toluidine blue. For immunohistochemistry deparaffinized sections were digested at RT for 10 min with $0.4 \%$ pepsin (w/v) (Sigma-Aldrich) in TBS$\mathrm{HCl}(\mathrm{pH}$ 2.0), blocked with $20 \%$ goat serum (v/v) (SigmaAldrich), and incubated overnight at $4^{\circ} \mathrm{C}$ with either type I collagen $(1: 100$, T59103R; Meridian) or type II collagen (1:100, MAB8887; Millipore) antibody. Immunoreactivity was detected by Alexa-488 goat anti-rabbit or Alex-594 goat antimouse secondary antibody (1:300, $1 \mathrm{~h}$; Invitrogen). Nuclei were visualized by DAPI (1:10,000; Invitrogen). IgG was used as the negative control. Images were collected using a $40 \times$ objective (Nikon Eclipse C1si).

\section{Tissue analysis}

The tissues were digested in papain $(40 \mu \mathrm{g} / \mathrm{mL}$ in $20 \mathrm{mM}$ ammonium acetate, $1 \mathrm{mM}$ ethylenediaminetetraacetic acid [EDTA], and $2 \mathrm{mM}$ DTT; Sigma-Aldrich) for $48 \mathrm{~h}$ at $65^{\circ} \mathrm{C}$ and assayed as described previously. ${ }^{2}$ Briefly, DNA content was quantified by Hoechst dye 33258 assay (Polysciences, Inc.) and fluorometry (excitation $\lambda=365 \mathrm{~nm}$ and emission $\lambda=458 \mathrm{~nm}$ ). Proteoglycan (PG) content was estimated by the dimethylmethylene blue dye binding assay $(\lambda=525 \mathrm{~nm})$ (Polysciences, Inc.). Collagen content was quantified by chloramine-T/Ehrlich's reagent assay $(\lambda=560 \mathrm{~nm})$. 
Table 1. List of Sequences of Primers Used in Quantitative PCR

\begin{tabular}{lll}
\hline Gene & \multicolumn{1}{c}{ Forward $\left(5^{\prime}\right)$} & \multicolumn{1}{c}{ Reverse $\left(5^{\prime}\right)$} \\
\hline Sox6 & tcctggcagcgcatgatgaaca & agcaaggtccatttgctgccgt \\
Sox9 & acacacagctcactcgaccttg & agggaattctggttggtcctct \\
Col1a1 & cggctcctgctcctctac & cacacgtctctctcggtcatggta \\
Col2a1 & gtgtcacggccaggatgtc & gcagaggacagtccagtgt \\
Co19a1 & aacgacggaggccttacagacgg & aattctggagaacttgcgg \\
Co110al & tccaaaatacaggtctgagc & cctgttaattgtcagaacag \\
Col11al & acagttgtgagtgcgggggct & tcccagagccaccgtttcgt \\
TenacinC & aagtcatccggcacaagcagca & acgtgattgaacaccaccggct \\
Wnt9a & acaactcgtgggtgtgaaggt & tcgtacttgtgcttcaggcgct \\
Gdf5 & tggtgttggccgcaccaagaa & aagttgacatgcagcgccttcc \\
Gli3 & tcggccagatgtcagcgagaaa & tgtggctgcatagtgactgcgt \\
Aggrecan & tgggactgaagttcttggaga & gcgagttgtcatggtctgaa \\
COMP & tgcctgtgacaactgtcctcagaa & ttgtctaccaccttgtccgcatca \\
CD44 & gatccacccaattccatctgt & aaccgcgagaatcaaagccaa \\
IR & tccgcacactcggtttctcctt & agggttcaaaccttgcagggca \\
IGF1R & ccgagttccagagctgtgcagtta & gatggtccacactgggcaagacc \\
\hline
\end{tabular}

PCR, polymerase chain reaction.

\section{RNA isolation and relative quantitative PCR}

Total RNA was extracted with RNeasy ${ }^{\circledR}$ kit (Qiagen) and reverse transcribed with 200 units of SuperscriptIII (Invitrogen). SYBR green dye I, $0.2 \mu \mathrm{M}$ of primers (Table 1), and realplex2 Master cycler (Eppendorf) were used for relative quantitative PCR (qPCR).

\section{Radiolabeling}

Synthesis of collagens and PGs was evaluated by assaying the incorporation of $\left[{ }^{3} \mathrm{H}\right]$-proline and $\left[{ }^{35} \mathrm{~S}_{-}-\mathrm{SO}_{4}(2 \mu \mathrm{Ci}\right.$; PerkinElmer), respectively, 3 days after cell seeding. Cells were labeled for $24 \mathrm{~h}$, washed in PBS, and digested in papain as described previously. Collagen and PG were precipitated from the media with $70 \%$ ammonium sulfate or $100 \%$ cold ethanol, respectively, and centrifuged at $14,000 \mathrm{rpm}$ for $30 \mathrm{~min}$ at $4^{\circ} \mathrm{C}$. Pellets were washed with cold $70 \%$ ethanol, and centrifuged for $10 \mathrm{~min}$ at $14,000 \mathrm{rpm}$. The precipitated collagen was resuspended in $10 \%$ sodium dodecyl sulfate (SDS) and the PGs were solubilized in $4 \mathrm{M}$ guanidine hydrochloride and quantified using a $\beta$-scintillation counter (Beckman Coulter).

To determine glucose uptake, cells were cultured in SF3DHG, SF3D-low glucose content media (LG), or SC3D-HG for $24 \mathrm{~h}$; serum or ITS and glucose starved for $2 \mathrm{~h}$; and then incubated with $\left[{ }^{3} \mathrm{H}\right]$-deoxyglucose $\left(2 \mathrm{D}-\mathrm{G}{ }^{3} \mathrm{H} ; 10 \mu \mathrm{Ci} / \mathrm{mL}\right)$ for $30 \mathrm{~min}$ at $37^{\circ} \mathrm{C}$. The cultures were rinsed with PBS, and digested with papain $(40 \mu \mathrm{g} / \mathrm{mL})$ for $24 \mathrm{~h}$. Glucose uptake was quantified by measuring ${ }^{3} \mathrm{H}$ incorporation using a $\beta$-scintillation counter and normalized to DNA content.

\section{Immunoblotting}

Cells were harvested in RIPA buffer containing 1\% NP40, $0.1 \%$ SDS, 2 mM EDTA, $0.5 \%$ sodium deoxycholate (NaDC), and $150 \mathrm{mM} \mathrm{NaCl}$ in $50 \mathrm{mM}$ Tris (pH 7.8). Fifteen micrograms of protein was separated on $12 \%$ SDS-polyacrylamide gel electrophoresis, and transferred to a nitrocellulose membrane by iblot (Invitrogen). Membranes were blocked in 1\% milk for $1 \mathrm{~h}$ and incubated with antibodies reactive with Sox9 (1:1000, ab3697; Abcam), $\beta$-actin (1:2000, A5441; Sigma-Aldrich), Shc, pSHC, pAKT, AKT, pERK, or ERK (1:1000, respectively, 2432, 2434, 9611s, 9272, 9102, and 9101; Cell Signalling) overnight. Washed membranes were incubated with HRPconjugated secondary antibodies for $1 \mathrm{~h}$ and immunoreactivity was detected by ECL + (GE Healthcare).

\section{Co-immunoprecipitation}

Lysis buffer containing 1\% Triton X-100, 10\% glycerol, $137 \mathrm{mM} \mathrm{NaCl}, 1 \mathrm{mM}$ Na-orthovanadate, and $1 \times$ protease inhibitors (Complete mini; Roche) was used to extract protein. Cell lysates $(120 \mu \mathrm{g})$ were centrifuged at $15,000 \mathrm{~g}$ for $20 \mathrm{~min}$ and the supernatant was precleared with protein $\mathrm{A} / \mathrm{G}$ beads (Millipore) for $2 \mathrm{~h}$ and then incubated for $4 \mathrm{~h}$ with antibody reactive with Sox9 (1:1000, ab3697; Abcam) at $4^{\circ} \mathrm{C}$. The immune complex was harvested by incubation with Protein $\mathrm{A} / \mathrm{G}$ beads overnight at $4^{\circ} \mathrm{C}$. Beads were washed in lysis buffer and boiled for $10 \mathrm{~min}$ in mercaptoethanol containing sample buffer and immunoblotted (see immunoblotting section) with antibodies reactive with Sox6 and Med12 (1:1000, ab66316 and ab49053; Abcam).

\section{Chromatin immunoprecipitation}

Chromatin immunoprecipitation (ChIP)-qPCR analysis was carried out using genomic DNA. Briefly, 2-day-old cultures were crosslinked in situ with $0.75 \%$ formaldehyde and harvested in $50 \mu \mathrm{L} / 10^{6}$ cells of lysis buffer ( $50 \mathrm{mM}$ HEPES-KOH [pH 7.5], $140 \mathrm{mM} \mathrm{NaCl}, 1 \mathrm{mM}$ EDTA [pH 8.0], 1\% Triton X$100,1 \% \mathrm{SDS}$, and $0.1 \% \mathrm{NaDC})$. DNA was sheared by sonication at $25 \%$ amp ( $30 \mathrm{~s} \mathrm{ON}$ and $60 \mathrm{~s}$ OFF $20 \times$; Vibracell) to 250-900 bp fragments (confirmed by agarose gel); a fraction was used as input control. Twenty-five micrograms of DNA was diluted 1:10 in RIPA and immunoprecipitated with antibody reactive with Sox 9 and Protein A/G beads overnight at $4^{\circ} \mathrm{C}$. Samples were reverse crosslinked in the presence of $5 \mu \mathrm{L}$ Proteinase $\mathrm{K}$ at $65^{\circ} \mathrm{C}$ overnight. Co-immunoprecipitated DNA was purified with equal volume of 1:1 phenol:chloroform and washed with $3 \times$ volume of ethanol containing $10 \mu \mathrm{L}$ of glycogen $(5 \mathrm{mg} / \mathrm{mL})$. The air-dried pellet was resuspended in $100 \mu \mathrm{L}$ of water; qPCR with Col2al primers (f-5' TTCCAGATGGGG CTGAAACGCT, r-5' TGGGGCTTTTCTCGAGCACACA) located in the Sox 9 binding region was carried out (see Table 1).

\section{Statistical analysis}

Each experiment was done using cartilage tissues obtained from a single animal. The results are expressed as the mean of three to five independent experiments. Each condition was done in triplicate. Pearson's chi-square test was used to verify the Gaussian distribution and independence of the data. Oneway analysis of variance followed by Tukey's post hoc test was used for all pair-wise comparisons between groups. $p$-Values $\leq 0.05$ were considered to be statistically significant. Data are presented as mean with $95 \%$ confidence interval $(95 \% \mathrm{CI})$.

\section{Results}

\section{Passaged chondrocytes lose their chondrocytic phenotype and do not form cartilage tissue in $3 D$ culture in the presence of serum containing media}

Cell passaging in ML culture resulted in an increase in cell number but cells lost their polygonal shape and acquired a spindled morphology (Fig. 1A). Gene expression analysis after each passage showed progressive loss of Col2al, Sox9, aggrecan, and COMP, and gain of Collal expression. In addition 


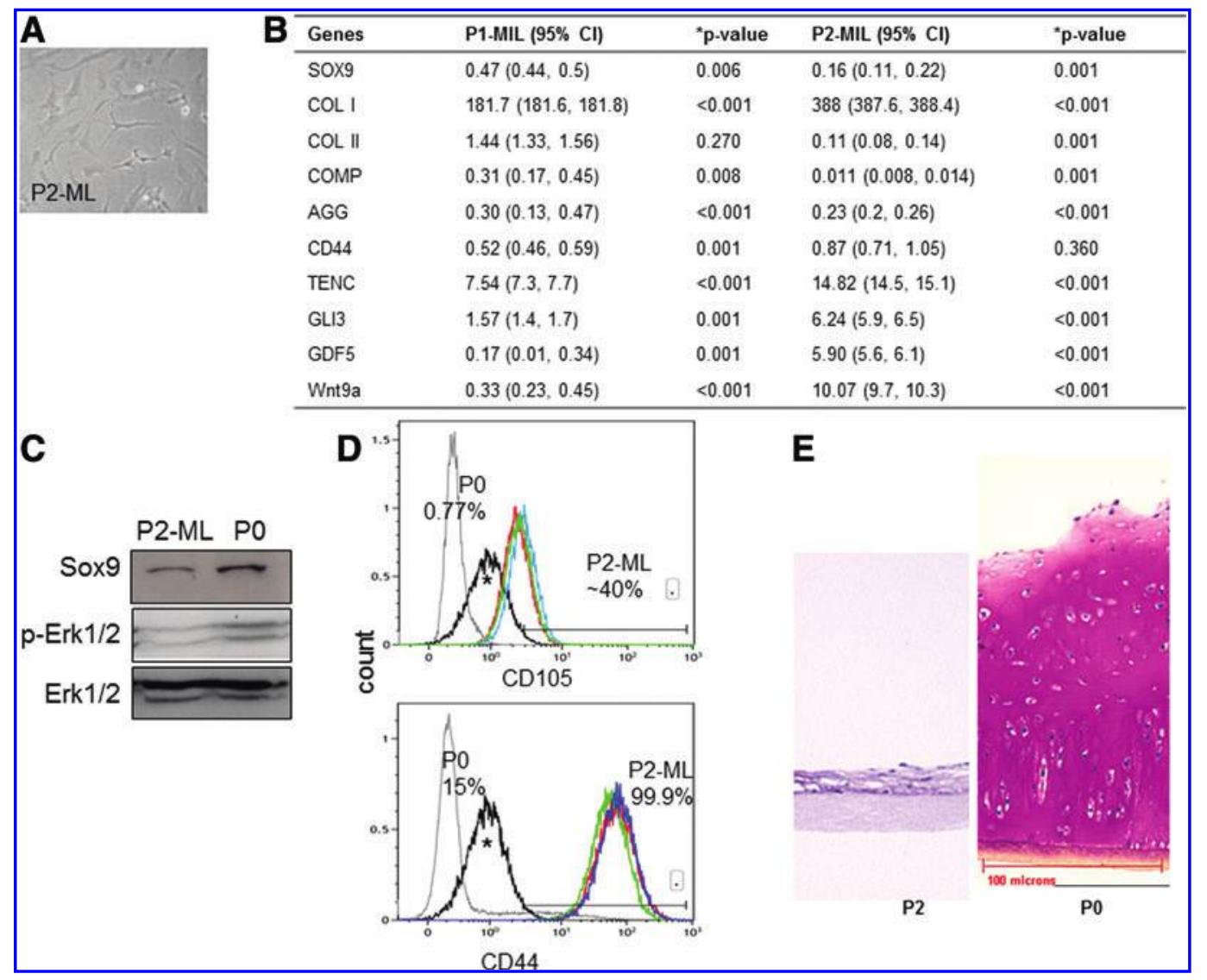

FIG. 1. Loss of chondrocytic phenotype in culture-expanded cells. (A) Spindle-shaped morphology of P2 cells in monolayer (ML). $n=5$ experiments. (B) Gene expression profile of cells after each passage compared with P0 levels. A significant increase in Colla1 was observed after the first passage along with downregulation of cartilage-associated gene aggrecan and cartilage oligomeric matrix protein $(C O M P)$ whereas a decrease in Col2al was seen only after the second passage. Genes expressed by interzone cells-TenacinC, Gli3, GDF5, and Wnt9a-were significantly upregulated in P2 cells compared with P0 or P1 cells. Data are expressed as mean with the uncertainty estimated by $95 \%$ confidence interval (95\% CI; lower and upper limits are within brackets). $n=5$ experiments. P0, primary chondrocytes; P1-ML or P2-ML, passage 1 or 2 cells, respectively, harvested from ML. (C) Immunoblot analysis showed a decrease in Sox9 and p-ERK-1/2 levels in P2-ML compared with P0. $n=3$ experiments. (D) FACS analysis of surface markers showed $40 \% \mathrm{CD} 105^{+}$and $99 \% \mathrm{CD} 4^{+}$cells after two passages. $n=3$ experiments. ${ }^{\wedge}$ Nonspecific background staining in P2 cells. (E) Photomicrographs of toluidine-blue-stained tissues formed by P2 cells cultured in serum containing three-dimensional conditions (SC3D) for 4 weeks show loss of capacity to accumulate cartilaginous matrix by P2 cells unlike P0 that retained abundant proteoglycan (PG)-rich matrix. $n=5$ experiments. $\lceil$ Collagen-type-II-coated membrane insert. Color images available online at www.liebertpub.com/tea

the cells showed a significant increase in the expression of interzone cell markers-Gli3, tenascinC, Gdf5, and Wnt9a (Fig. 1B). Sox 9 and p-ERK-1/2 protein levels were decreased in passaged cells (P2) as determined by western blot analysis (Fig. 1C). Flow cytometry showed that $>99 \%$ of $\mathrm{P} 2$ cells expressed the hyaluronan receptor $\mathrm{CD} 44^{+}$and nearly $40 \%$ of the cells were $\mathrm{CD} 05^{+}$, a marker of marrow stromal cells (Fig. 1D). The P2 cells were placed in SC3D, and histological analysis after 4 weeks showed that they were unable to form cartilage tissue, in contrast to the PG-rich tissue formed by the primary (P0) chondrocytes under the same conditions (Fig. 1E).

\section{Passaged chondrocytes undergo phenotype reversal and form cartilage tissue in $3 D$ culture in serum-free media}

In SF3D, P2 reacquired the ability to form cartilage tissue as compared with cells grown in the presence of serum. After 4 weeks of culture P2 had accumulated abundant hyaline carti- laginous extracellular matrix that contained sulfated PGs (Fig. $2 \mathrm{~A}$ ) and type II collagen. Type I collagen was not detected by immunostaining (Fig. 2A). The amount of glycosaminoglycans was similar to that accumulated by $\mathrm{P} 0$ grown in SC3D culture (Fig. 2B). FACS analysis of the P2 at $48 \mathrm{~h}$ of SF3D culture revealed that only $25 \%$ of $\mathrm{P} 2$ in SF3D were CD $44^{+}$and $100 \%$ had lost CD105 antigen (Fig. 2C). Gene expression analysis at 3 weeks showed that Col2al, Sox9, aggrecan, and COMP were higher in SF3D compared with P2 cells grown in SC3D. Collal gene expression was lower in both conditions. Colloal was significantly lower in SF3D (Fig. 2D). CollOal levels were in the same range as that found in primary chondrocytes (data not shown). Cells grown under either culture conditions showed similar DNA content (Fig. 2E). Analysis of $\left[{ }^{3} \mathrm{H}\right]$-proline and $\left[{ }^{35} \mathrm{~S}\right]-\mathrm{SO}_{4}$ incorporation at day 2 of culture showed that $\mathrm{P} 2$ in SF3D synthesized and retained significantly more collagens. PG synthesis was higher in the SC3D culture conditions; however, significantly more PGs were retained in SF3D (Fig. 2E). This suggested that collagen synthesis and retention was important for 


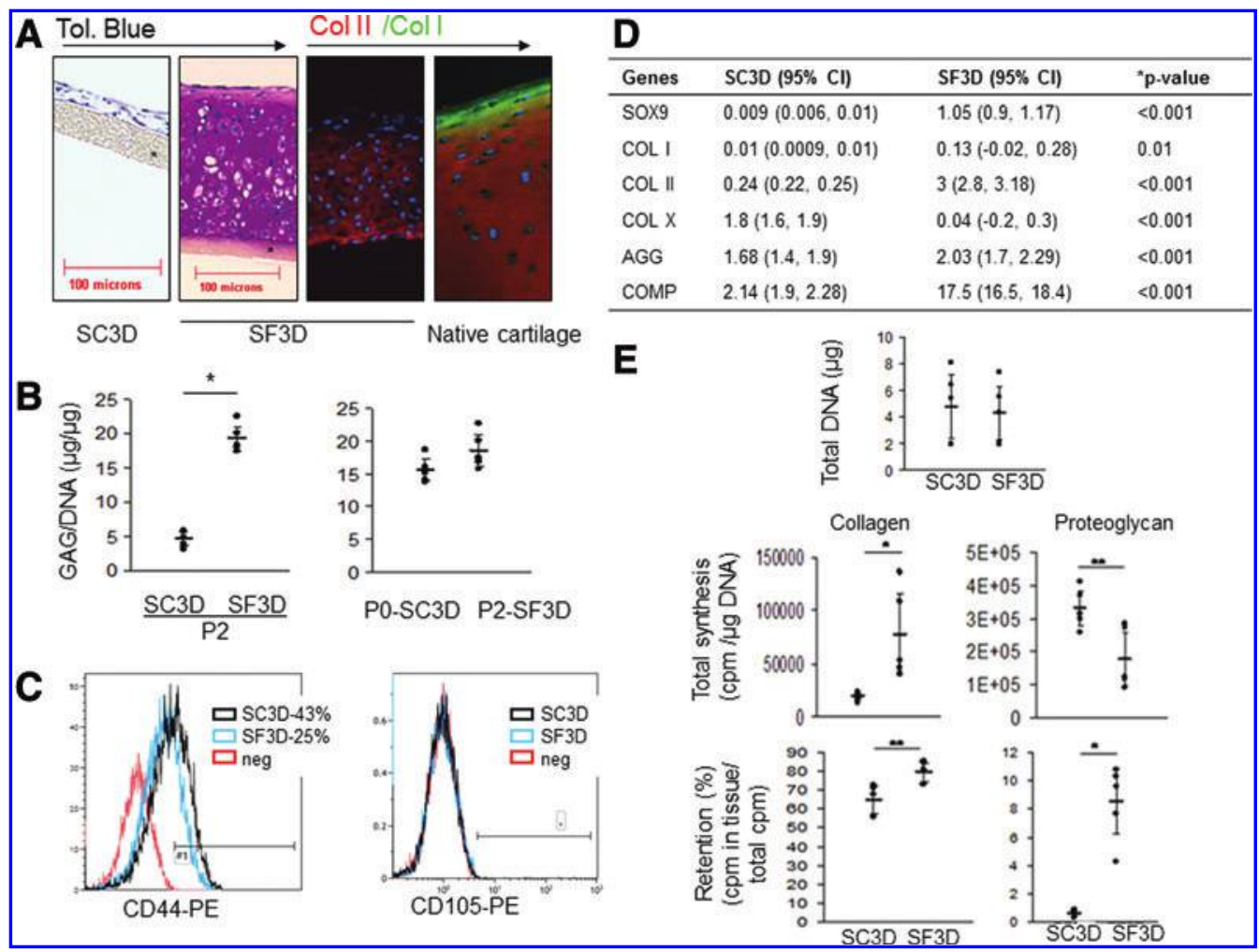

FIG. 2. Assessment of the tissue formed by the P2 cells in SF3D. (A) Photomicrographs of 4-week-old tissue formed by $\mathrm{P} 2$ cells cultured in SC3D (serum containing media) show no matrix accumulation (far left panel) while growth in SF3D (serum-free media) shows accumulation of matrix rich in PGs (toluidine blue; second panel). SF3D-generated tissue stained positively for collagen type II (red) and was predominantly negative for collagen type I (green) (third panel) similar to native cartilage tissue stained with the same antibodies (fourth panel). $n=5$ experiments. (B) More PGs (glycosaminoglycan) were accumulated in tissues formed by P2 in SF3D than in SC3D (left) and was comparable to that accumulated by P0 cells in SC3D (right) at 4 weeks of culture. Each dot represents an independent experiment; horizontal lines represent mean and 95\% CI. $n=5$ experiments. (C) FACS analysis after 2 days of culture shows complete loss of CD105 in both the conditions while only $25 \%$ of the cells retained CD44 in SF3D in comparison to $43 \%$ in SC3D. $n=3$ experiments. (D) After 3 weeks the gene expression profile relative to freshly harvested P2-ML cells also shows that P2 in SF3D expressed significantly higher cartilage-related genes and lower Colloal expression compared with the cells in SC3D. Data are shown as mean with the uncertainty estimated by $95 \%$ CI (lower and upper limits are within brackets). $n=3$ experiments. (E) Total DNA content of tissues was similar between the conditions. At $48 \mathrm{~h}, \mathrm{P} 2$ in SF3D synthesized and retained more collagen and, although PG retention was higher in SF3D, more was synthesized in SC3D. ๆ Collagen-type-II-coated membrane inserts; all images are of the same magnification. Each dot represents the mean of an independent experiment; horizontal lines represent mean and $95 \%$ CI. $n=5$ experiments. ${ }^{*} p \leq 0.01$, $* * p \leq 0.05$ compared with SC3D. Color images available online at www.liebertpub.com/tea

tissue formation and this parameter was used as the marker for short-term assessments in subsequent experiments.

\section{Redifferentiation is insulin, dexamethasone, and HG dependent}

To evaluate the contribution of the individual media components, P2 cells were cultured under various media conditions and histological analysis at 4 weeks of culture showed that insulin, dexamethasone (synthetic glucocorticoid), and high glucose content DMEM were all essential for cartilage growth (Fig. 3). In contrast to the thin fibrous layer of tissue formed by the P2 cells grown in the absence of insulin or in low glucose, $\mathrm{P} 2$ cells grown in insulin and HG resulted in thick hyaline-like cartilage tissue rich in PGs. The presence of dexamethasone also contributed to cartilage formation but was insufficient in the absence of $\mathrm{HG}$ or insulin to support generation of this tissue (Fig. 3). IGF-1 could not replace insulin as no tissue formed in the presence of this growth factor, even when $\mathrm{HG}$ and dexamethasone were present. The serum substitute ITS + was replaceable by insulin. DNA content was measured after $48 \mathrm{~h}$ of culture and was similar in both HG and LG culture conditions, indicating that there were no differences in cell attachment and survival (data not shown).

\section{Characterization of cells during early redifferentiation}

Redifferentiation was evident as early as $48 \mathrm{~h}$ as shown by upregulation of Sox9 and Col2al gene expression and downregulation of Collal (Fig. 4A). Western blot analysis of protein extracts from 48-h-old SF3D cultures shows increased phosphorylation of the insulin/IGF signaling molecules Shc and AKT (Fig. 4B). Based on these results the remaining analyses were done within $48 \mathrm{~h}$. Quantification of 


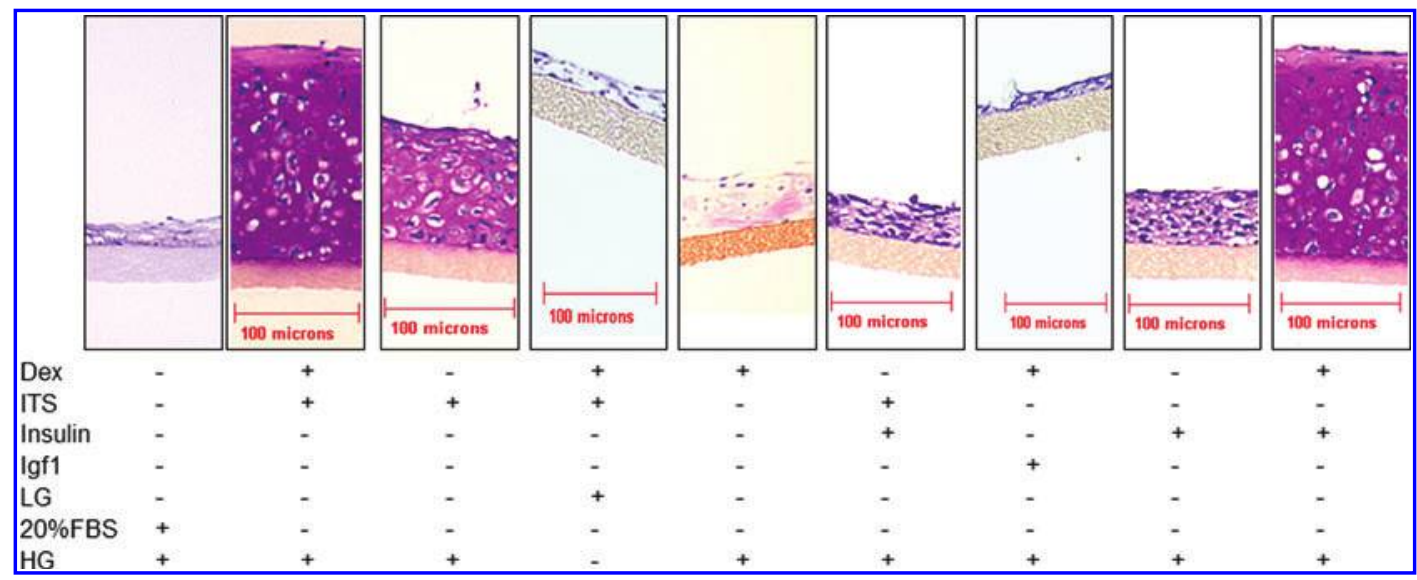

FIG. 3. Insulin, HG, and Dex are essential components of SF3D. Photomicrographs of the histological appearance of tissue formed by P2 under different media conditions after 4 weeks of culture. Dex, dexamethasone; HG, high glucose content media; LG, low glucose content media. I Collagen-type-II-coated membrane inserts. $n=3$ experiments. Color images available online at www.liebertpub.com/tea

intracellular glucose levels showed that cells exhibited glucose uptake although those cultured in low-glucose media had significantly higher levels than in HG-SF or SC (20\% FBS) condition (Fig. 4C).

\section{Insulin signaling is involved in Sox9-mediated chondrogenic differentiation}

The role of insulin in this process was evaluated using pharmacological agents known to inhibit insulin signaling. Synthesis of collagens was significantly reduced by HNMPA-
(AM3) treatment, a drug known to inhibit the IR (Fig. 5A). PG synthesis was also inhibited by this treatment (data not shown). We confirmed by qPCR and immunoblotting that HNMPA-(AM3) treatment downregulated IR gene [control=35 $(33.9,36)$ vs. HNMPA-(AM3) treated $=14(13.4$, 14.5; mean $\pm 95 \%$ CI)] and protein levels (Fig. 5B) and that the activity of Shc, a signaling pathway downstream of IR, was also reduced after IR inhibition (Fig. 5C). We then examined the levels of ERK/MAPK that lies downstream of Shc. Phosphorylation of 44-kDa ERK-1 subunit was unaffected after inhibition; however, the 42-kDa ERK-2 subunit was

\begin{tabular}{|lllll}
\hline A & & & & \\
\hline Genes & 24 hrs $(95 \% \mathrm{Cl})$ & P-value & 48 hrs $(95 \% \mathrm{Cl})$ & p-value \\
\hline SOX6 & $1.6(1.5,1.7)$ & 0.048 & $4.1(4.01,4.19)$ & 0.003 \\
SOX9 & $4.5(4.2,4.8)$ & $<0.001$ & $3.6(3.58,3.62)$ & 0.004 \\
COLI & $0.9(0.2,1.5)$ & 0.314 & $0.9(0.54,1.26)$ & 0.001 \\
COL II & $1.5(1.45,1.55)$ & 0.024 & $7.1(6.89,7.31)$ & $<0.001$ \\
\hline
\end{tabular}
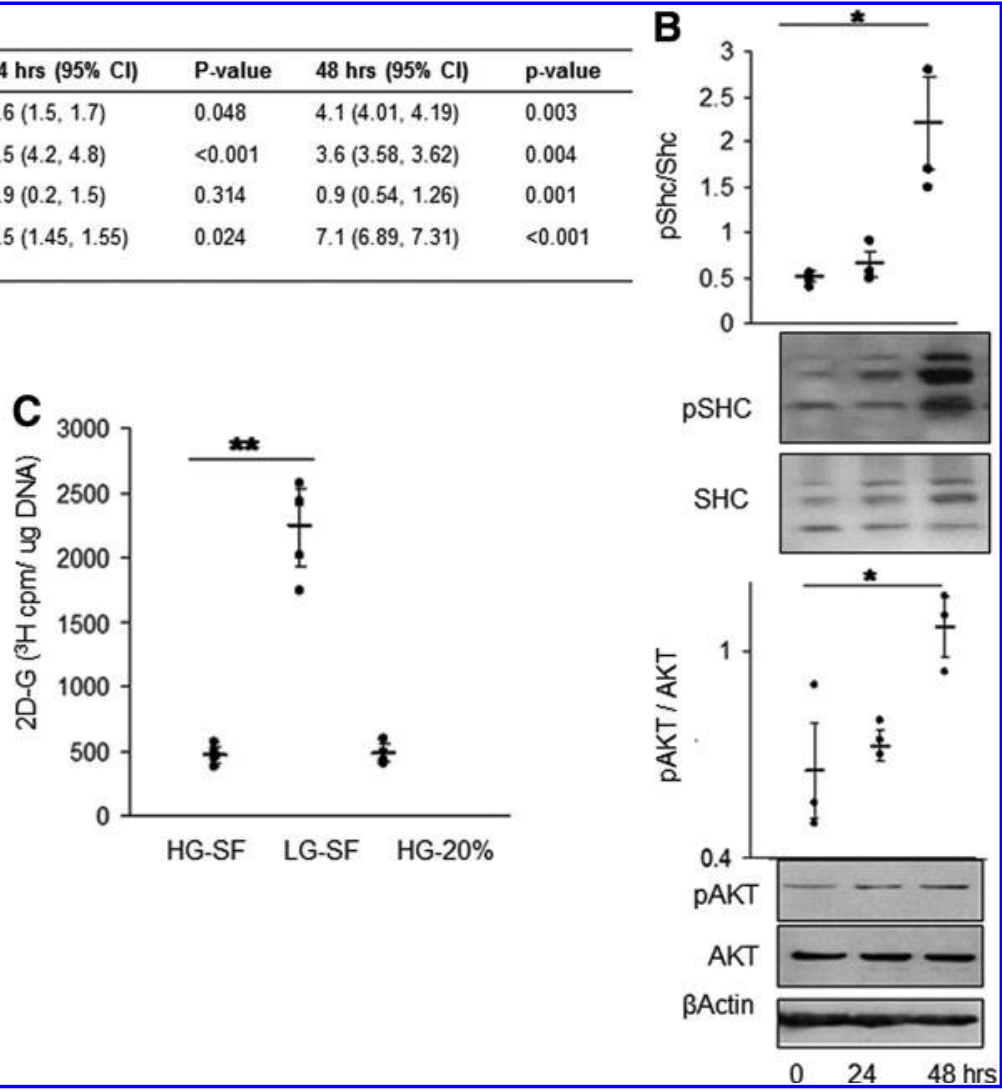

FIG. 4. Redifferentiation of $\mathrm{P} 2$ in SF3D. (A) Quantitative PCR analysis showed that cartilage-differentiationassociated genes in P2 cells were upregulated early. Collal was downregulated by $48 \mathrm{~h}$ of culture when compared with freshly harvested P2-ML cells. Data are shown as mean with $95 \%$ CI (lower and upper limits are within brackets). $n=3$ experiments. (B) Representative immunoblots and densitometry of extracts from redifferentiating $\mathrm{P} 2$ cells in SF3D over the first 2 days of culture showed upregulation of insulin/IGF-pathway-related signaling molecules. (C) Glucose assay demonstrated higher uptake as indicated by increased intracellular glucose $\left(2 \mathrm{D}-\mathrm{G}{ }^{3} \mathrm{H}\right)$ by $\mathrm{P} 2$ cells cultured under LG serum-free conditions. HG-SF, serum-free high glucose content medium containing ITS; LG-SF, low glucose content media containing ITS; HG-20\%, high glucose content medium with $20 \%$ fetal bovine serum. Each dot represents the mean of an independent experiment; horizontal lines represent mean and $95 \%$ CI. $n=5$ experiments. $* p \leq 0.01, * * p \leq 0.05$. 


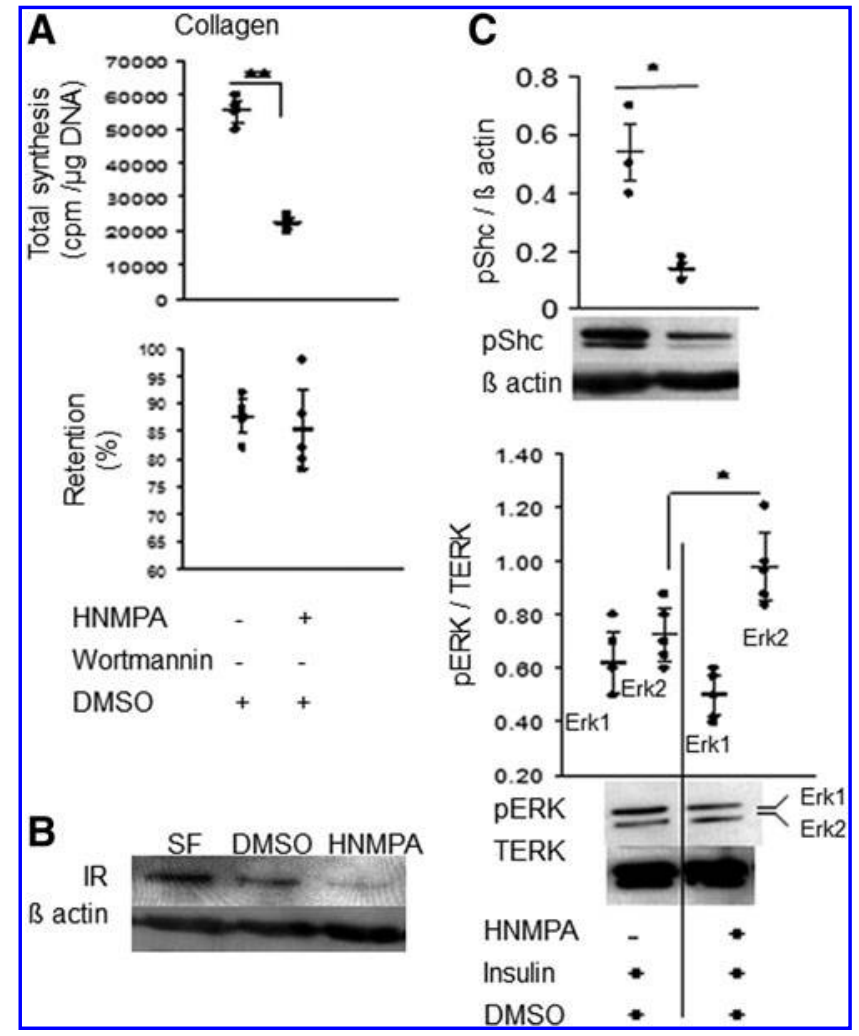

FIG. 5. Effect of inhibition of insulin receptor (IR). (A) Radioisotope incorporation studies show inhibition of total collagen synthesis but no change in \% retention with HNMPA-(AM3) treatment. (B) Immunoblotting shows that HNMPA-(AM3) decreased IR protein levels. $n=3$ experiments. (C, D) Immunoblots followed by densitometry shows downregulation of Shc phosphorylation (C) and upregulation of 42-kDa ERK (D) in HNMP-treated cells. Each dot represents the mean of an independent experiment; horizontal lines represent mean and $95 \%$ CI. $n=3$ or 5 experiments. $* p \leq 0.01, * * p \leq 0.05$ compared with DMSO control.

significantly more phosphorylated, potentially implicating ERK-2 as a mediator of the insulin effect (Fig. 5D).

Both the gene and protein levels of sox 9 and -6 were also significantly decreased by HNMPA-(AM3) treatment (Fig. $6)$. Gene expression analysis of P2 showed downregulation of cartilage-related collagens, Col2al and colllal (Fig. 6B). The Sox 9 transcription complex likely remained unaffected as Sox6 and Med12 co-immunoprecipitated with Sox9 after HNMPA-(AM3) treatment (Fig. 6C). Given the decreased levels of Sox 9 protein, we determined whether Sox 9 binding to its target gene Col2al was affected. ChIP analysis showed that Sox9 binds to the Col2al gene at similar levels as in primary cells cultured in chondrogenic condition but the binding was significantly decreased in the treated P2 cells as would be expected given the decreased Sox9 levels (Fig. 6D). This suggested that insulin-regulated Sox9 levels influenced collagen synthesis by $\mathrm{P} 2$ cells.

\section{Discussion}

The data shows that chondrocytes serially passaged in ML culture (P2-ML) lost their chondrocyte phenotype and

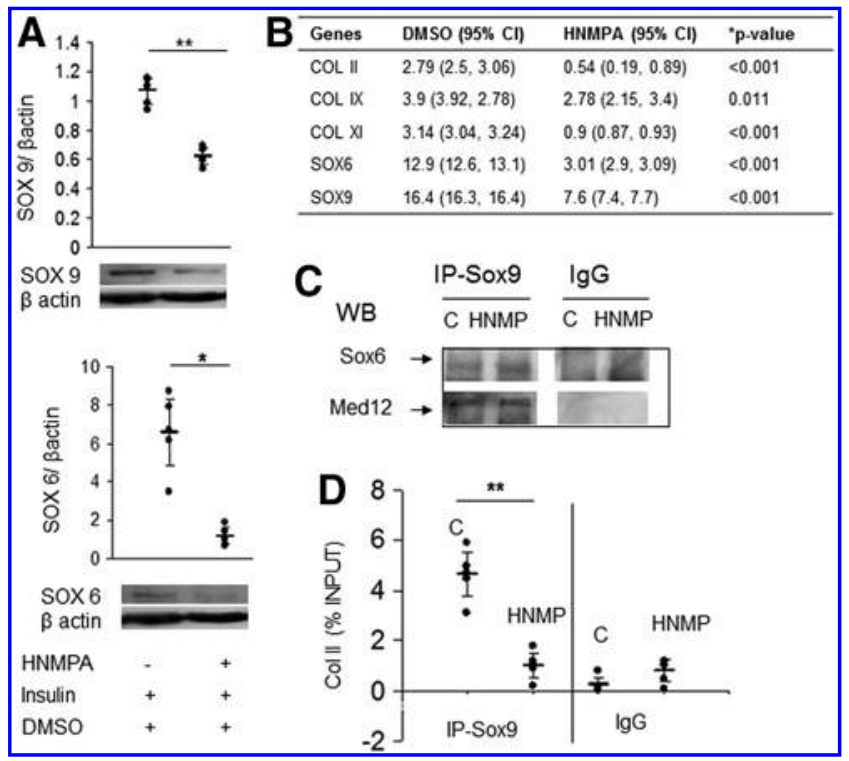

FIG. 6. Insulin mediates SOX9 levels and col $2 a$ expression. (A) Immunoblot and densitometry show that treatment with HNMPA-(AM3) leads to lower protein levels of Sox6 and Sox9 in P2 grown in SF3D. Each dot represents the mean of an independent experiment; horizontal lines represent mean and 95\% CI. $n=5$ experiments. (B) Gene expression also shows decreases in cartilage-associated genes after downregulating IR. Data are expressed relative to P2ML and are shown as mean with $95 \%$ CI (lower and upper limits). $* p \leq 0.01$, ** $p \leq 0.05 . n=3$ experiments. (C) Physical interaction of transcription factor Sox6 and binding protein MED12 with Sox9 is unaffected by HNMPA-(AM3) treatment as seen in co-IP analysis. $n=5$ independent experiments. (D) Chromatin immunoprecipitation extraction of Sox9-bound DNA showed decreased binding to Col2al after HNMPA-(AM3) treatment (IR downregulation). Each dot represents the mean of an independent experiment; horizontal lines represent mean and 95\% CI. $n=5$ experiments. ${ }^{*} p \leq 0.01$. C, control; co-IP, co-immunoprecipitation.

acquired a "progenitor-like" phenotype with a gene expression profile suggestive of interzone cells. These cells could be redifferentiated to chondrocytes that form hyalinelike cartilage tissue using a defined serum-free culture system (SF3D) in the absence of exogenous factors, such as TGF $\beta$, as demonstrated by the ability of the cells to form cartilaginous hyaline tissue that appears qualitatively and quantitatively similar to the tissue formed by primary chondrocytes. $^{26}$ The redifferentiated cells showed elevated gene and protein levels of sox 9 and mRNA levels of the cartilaginous matrix molecules Col2al, COMP, and $a g-$ grecan and significantly lower Collal expression compared with P2-ML. Expression of Col10 was low in SF3D and immunostaining of tissue sections also did not show collagen type 10 in tissue formed in SF3D at 3 weeks (data not shown). These findings suggest that the redifferentiated chondrocytes have an articular phenotype. ${ }^{27}$ This redifferentiation in $\mathrm{HG}$ appears to be regulated in part by a signal transduction cascade involving insulin that upregulated Sox 9 expression and modulated col $2 a$ expression and collagen synthesis. These findings are consistent with other studies that show that chondrocyte phenotype can be 
changed by modulating the microenvironment and/or culture conditions. $10,26,28$

SF3D culture provides a sufficient chondro-conducive environment for the P2 cells that obviates the need for addition of exogenous TGF $\beta$ family molecules. Interestingly, SF3D does not invoke the same response (formation of cartilage tissue) in primary chondrocytes (Supplementary Fig. S1; Supplementary Data are available online at www.liebertpub.com/tea). To our knowledge, almost every study on redifferentiation of passaged chondrocytes has supplemented media with BMP, TGF $\beta$, or some other type of growth factor. ${ }^{11,29}$ In this SF3D system addition of TGF $\beta 3$ or inhibition of TGFß3 signaling (SB431542) showed no effect on collagen synthesis by $\mathrm{P} 2$ cells further confirming the independence of this redifferentiation process on exogenous TGF $\beta$ (Supplementary Fig. S2) and that the cells were likely not producing TGF $\beta$ endogenously. It is possible that $\mathrm{P} 2$ cells in SF3D do not require exogenous growth factors, such as TGF $\beta$, due to their unique progenitor-like phenotype with interzone features. A number of previous studies have also shown that passaged cells acquire some features of either MSCs $^{5,6,30}$ or interzone cells, such as GDF5 protein expression. $^{31}$ This type of differentiation may impart a unique phenotype on bovine P2 cells, which may explain their independence of growth factors. Additional studies are needed to further characterize this phenotype.

While type-II-collagen-coated membranes are part of the SF3D system, the critical components of the DMEM media are insulin, dexamethasone, and high glucose. Since all the three media components are essential for the tissue formation, it is difficult to determine whether they support each other or act independently. Glucose levels are important as culturing in low glucose prevented cartilage tissue formation. The mechanism by which glucose regulates redifferentiation and matrix accumulation is not clear; especially as in this system glucose uptake by the redifferentiating cells was greater in the presence of low-glucose media. HG levels have been shown to promote chondrogenesis in MSCs by downregulating ERK and upregulating p38. Glucose can have other effects such as inducing $O$-glycosylation of EGF domains in $\mathrm{PGs}^{32,33}$ and macromolecular crosslinking that may alter matrix retention. ${ }^{34}$ Further studies will be required to delineate the role of glucose in this system. It is highly likely that dexamethasone acted by influencing matrix synthesis because the tissue formed by the cells cultured without dexamethasone contained much less matrix compared with the cells grown in the presence of dexamethasone (Fig. 3A). In support of this, blocking the effect of dexamethasone by treating cells with the glucocorticoid receptor antagonist led to decreased collagen and PG synthesis (data not shown). Interestingly, glucocorticoids have been shown to induce IR mRNA levels in other cell types. ${ }^{35}$

It was not entirely unexpected to find that insulin promotes chondrogenesis in passaged cells as this effect has been reported in other cell types, such as MSCs and the ATDC5 cells (mouse teratocarcinoma cell line that differentiates to chondrocytes in the presence of insulin). ${ }^{36,37}$ Proteomic studies suggest that insulin levels in FBS are much lower than that used in SF3D, which might explain why the presence of $20 \%$ FBS in SC3D was insufficient to promote matrix formation and accumulation by P2 cells. ${ }^{12}$ The involvement of insulin was supported by the observa- tion that treatment with HNMPA-(AM3) downregulated IR gene and protein levels, decreased sox 9 and -6 levels, and decreased synthesis of collagens. However, HNMPA-(AM3) is known to have other effects that could affect chondrogenesis. It can block prostaglandin synthesis by chondrocytes, ${ }^{38}$ which has been shown to affect chondrogenesis; hence, HNMPA-(AM3) could be working by mechanisms other than just modulating insulin effects. ${ }^{39}$ In our culture system insulin may act via Shc as the levels of pShc and downstream ERK-MAPK were modulated by HNMPA(AM3). In keeping with this observation microarray analysis during redifferentiation showed upregulation of Shc binding protein and PRKCSH (data not shown), both involved in Shc-mediated cascade. ${ }^{40}$ Interestingly only the ERK-2 $(42 \mathrm{kDa})$ and not the ERK-1 (44 kDa) subunit was affected by HMNPA-(AM3) treatment. Differential ERK-1/2 responses have been described by others as well. ${ }^{41,42}$ Several studies have shown that under physiological conditions ERK-2 can exist in predominantly a monomeric form (not dimerized to ERK-1) and is capable of regulating downstream signaling pathways. ${ }^{42}$ It was unexpected that ERK-2 levels would increase with IR inhibition by HNMPA-(AM3) treatment, but others have also observed that insulin can inhibit ERK, although in neuronal cells. ${ }^{43}$ Further, our results are consistent with those that have shown that high levels of ERK-2 inhibit differentiation in mouse embryonic stem cells. ${ }^{44}$

The data also suggests that the level of Sox 9 may be important for redifferentiation, which would be consistent with the study on liver cells where Sox 9 was shown to affect differentiation in a dose-dependent manner. ${ }^{45}$ In our system the redifferentiating cells have higher levels of Sox9 and subsequently higher association with Col2al on chromatin when compared with cells treated with HMNPA-(AM3), a condition that prevented the increase in collagen synthesis. In addition, this treatment-although affecting levels of sox9 and sox6-did not seem to affect the formation of the transcriptional complex, at least as investigated in this study, as the cotranscriptional molecules sox6 and Med12 were detected. This suggests that insulin-induced increases in Sox9 levels may be involved in the redifferentiation and/or accumulation of matrix by passaged cells.

In summary we describe a novel serum- and TGF $\beta$-free culture system that requires insulin, dexamethasone, and HG to generate hyaline-like cartilage tissue from passaged bovine chondrocytes grown in 3D on collagen-type-II-coated membrane inserts. This is an important step toward the ultimate goal of developing autologous, patient-specific tissue-engineered hyaline-like cartilage tissue suitable to use for repair/replacement of damaged articular cartilage. Further studies are required to translate what has been learnt from this SF3D study for use with human chondrocytes.

\section{Acknowledgments}

The authors thank Mr. Harry Bojarski and RydingRegency Meat Packers for providing bovine tissues. The project was supported by funding from U.S. Department of Defense. N.A. received a fellowship from the Samuel Lunenfeld Research Institute Special Opportunities Fund. J.I. was supported by OGSST, ON. C.E.B. was funded by BRAVO!/MHIRT and by NIH grant No. MD 001427. 


\section{Disclosure Statement}

No competing financial interests exist.

\section{References}

1. Holtzer, H., Abbott, J., Lash, J., and Holtzer, S. The loss of phenotypic traits by differentiated cells in vitro, I. Dedifferentiation of cartilage cells. Proc Natl Acad Sci U S A 46, 1533, 1960.

2. Gan, L., and Kandel, R.A. In vitro cartilage tissue formation by co-culture of primary and passaged chondrocytes. Tissue Eng 13, 831, 2007.

3. Kon, E., Filardo, G., Di Martino, A., and Marcacci, M. ACI and MACI. J Knee Surg 25, 17, 2012.

4. Mackay, A.M., Beck, S.C., Murphy, J.M., Barry, F.P., Chichester, C.O., and Pittenger, M.F. Chondrogenic differentiation of cultured human mesenchymal stem cells from marrow. Tissue Eng 4, 415, 1998.

5. Tallheden, T., Dennis, J.E., Lennon, D.P., Sjogren-Jansson, E., Caplan, A.I., and Lindahl, A. Phenotypic plasticity of human articular chondrocytes. J Bone Joint Surg Am 85-A(Suppl 2), 93, 2003.

6. Fickert, S., Fiedler, J., and Brenner, R.E. Identification of subpopulations with characteristics of mesenchymal progenitor cells from human osteoarthritic cartilage using triple staining for cell surface markers. Arthritis Res Ther 6, R422, 2004.

7. Pittenger, M.F., Mackay, A.M., Beck, S.C., Jaiswal, R.K., Douglas, R., Mosca, J.D., et al. Multilineage potential of adult human mesenchymal stem cells. Science 284, 143, 1999.

8. Bonaventure, J., Kadhom, N., Cohen-Solal, L., Ng, K.H., Bourguignon, J., Lasselin, C., et al. Reexpression of cartilage-specific genes by dedifferentiated human articular chondrocytes cultured in alginate beads. Exp Cell Res $\mathbf{2 1 2}$, 97, 1994.

9. Chaipinyo, K., Oakes, B.W., and van Damme, M.P. Effects of growth factors on cell proliferation and matrix synthesis of low-density, primary bovine chondrocytes cultured in collagen I gels. J Orthop Res 20, 1070, 2002.

10. Quarto, R., Campanile, G., Cancedda, R., and Dozin, B. Thyroid hormone, insulin, and glucocorticoids are sufficient to support chondrocyte differentiation to hypertrophy: a serum-free analysis. J Cell Biol 119, 989, 1992.

11. Narcisi, R., Quarto, R., Ulivi, V., Muraglia, A., Molfetta, L., and Giannoni, P. TGF beta-1 administration during ex vivo expansion of human articular chondrocytes in a serum-free medium redirects the cell phenotype toward hypertrophy. J Cell Physiol 227, 3282, 2012.

12. Zheng, X., Baker, H., Hancock, W.S., Fawaz, F., McCaman, M., and Pungor, E., Jr. Proteomic analysis for the assessment of different lots of fetal bovine serum as a raw material for cell culture. Part, I.V. Application of proteomics to the manufacture of biological drugs. Biotechnol Prog 22, 1294, 2006.

13. Sensebe, L., Krampera, M., Schrezenmeier, H., Bourin, P., and Giordano, R. Mesenchymal stem cells for clinical application. Vox Sang 98, 93, 2010.

14. Siddle, K. Signalling by insulin and IGF receptors: supporting acts and new players. J Mol Endocrinol 47, R1, 2011.

15. Uhles, S., Moede, T., Leibiger, B., Berggren, P.O., and Leibiger, I.B. Selective gene activation by spatial segregation of insulin receptor B signaling. FASEB J 21, 1609, 2007.
16. Ikegami, D., Akiyama, H., Suzuki, A., Nakamura, T., Nakano, T., Yoshikawa, H., et al. Sox9 sustains chondrocyte survival and hypertrophy in part through Pik3ca-Akt pathways. Development 138, 1507, 2011.

17. Rosa, S.C., Rufino, A.T., Judas, F., Tenreiro, C., Lopes, M.C., and Mendes, A.F. Expression and function of the insulin receptor in normal and osteoarthritic human chondrocytes: modulation of anabolic gene expression, glucose transport and GLUT-1 content by insulin. Osteoarthritis Cartilage 19, 719, 2011.

18. Malafaya, P.B., Oliveira, J.T., and Reis, R.L. The effect of insulin-loaded chitosan particle-aggregated scaffolds in chondrogenic differentiation. Tissue Eng Part A 16, 735, 2009.

19. Bi, W., Huang, W., Whitworth, D.J., Deng, J.M., Zhang, Z., Behringer, R.R., et al. Haploinsufficiency of Sox 9 results in defective cartilage primordia and premature skeletal mineralization. Proc Natl Acad Sci U S A 98, 6698, 2001.

20. Lefebvre, V., Huang, W., Harley, V.R., Goodfellow, P.N., and de Crombrugghe, B. SOX9 is a potent activator of the chondrocyte-specific enhancer of the pro alpha1(II) collagen gene. Mol Cell Biol 17, 2336, 1997.

21. Akiyama, H., and Lefebvre, V. Unraveling the transcriptional regulatory machinery in chondrogenesis. J Bone Miner Metab 29, 390, 2011.

22. Zhao, Q., Eberspaecher, H., Lefebvre, V., and De Crombrugghe, B. Parallel expression of Sox 9 and Col2a1 in cells undergoing chondrogenesis. Dev Dyn 209, 377, 1997.

23. de Crombrugghe, B., Lefebvre, V., Behringer, R.R., Bi, W., Murakami, S., and Huang, W. Transcriptional mechanisms of chondrocyte differentiation. Matrix Biol 19, 389, 2000.

24. Oh, C.D., Maity, S.N., Lu, J.F., Zhang, J., Liang, S., Coustry, F., et al. Identification of SOX9 interaction sites in the genome of chondrocytes. PLoS One 5, e10113, 2010.

25. Baltensperger, K., Lewis, R.E., Woon, C.W., Vissavajjhala, P., Ross, A.H., and Czech, M.P. Catalysis of serine and tyrosine autophosphorylation by the human insulin receptor. Proc Natl Acad Sci U S A 89, 7885, 1992.

26. Ahmed, N., Gan, L., Nagy, A., Zheng, J., Wang, C., and Kandel, R.A. Cartilage tissue formation using redifferentiated passaged chondrocytes in vitro. Tissue Eng Part A 15, 665, 2009.

27. Leung, V.Y., Gao, B., Leung, K.K., Melhado, I.G., Wynn, S.L., Au, T.Y., et al. SOX9 governs differentiation stagespecific gene expression in growth plate chondrocytes via direct concomitant transactivation and repression. PLoS Genet 7, e1002356, 2011.

28. Rosenzweig, D.H., Chicatun, F., Nazhat, S.N., and Quinn, T.M. Cartilaginous constructs using primary chondrocytes from continuous expansion culture seeded in dense collagen gels. Acta Biomater 9, 9360, 2013.

29. Narcisi, R., Signorile, L., Verhaar, J.A., Giannoni, P., and van Osch, G.J. TGFbeta inhibition during expansion phase increases the chondrogenic re-differentiation capacity of human articular chondrocytes. Osteoarthritis Cartilage 20, 1152, 2012.

30. Polacek, M., Bruun, J.A., Elvenes, J., Figenschau, Y., and Martinez, I. The secretory profiles of cultured human articular chondrocytes and mesenchymal stem cells: implications for autologous cell transplantation strategies. Cell Transplant 20, 1381, 2010.

31. Schlegel, W., Albrecht, C., Eckl, P., Freudenthaler, H., Berger, A., Vecsei, V., et al. Dedifferentiation of human articular chondrocytes is associated with alterations in 
expression patterns of GDF-5 and its receptors. J Cell Mol Med 13, 3398, 2009.

32. Han, Y.S., Bang, O.S., Jin, E.J., Park, J.H., Sonn, J.K., and Kang, S.S. High dose of glucose promotes chondrogenesis via PKCalpha and MAPK signaling pathways in chick mesenchymal cells. Cell Tissue Res 318, 571, 2004.

33. Whitworth, G.E., Zandberg, W.F., Clark, T., and Vocadlo, D.J. Mammalian notch is modified by D-Xyl-alpha1-3D-Xyl-alpha1-3-D-Glc-beta1-O-Ser: implementation of a method to study O-glucosylation. Glycobiology 20, 287, 2009.

34. Mentink, C.J., Hendriks, M., Levels, A.A., and Wolffenbuttel, B.H. Glucose-mediated cross-linking of collagen in rat tendon and skin. Clin Chim Acta 321, 69, 2002.

35. Mamula, P.W., McDonald, A.R., Brunetti, A., Okabayashi, Y., Wong, K.Y., Maddux, B.A., et al. Regulating insulinreceptor-gene expression by differentiation and hormones. Diabetes Care 13, 288, 1990.

36. Laron, Z. Insulin - a growth hormone. Arch Physiol Biochem 114, 11, 2008.

37. Phornphutkul, C., Wu, K.Y., and Gruppuso, P.A. The role of insulin in chondrogenesis. Mol Cell Endocrinol 249, 107, 2006.

38. Jacques, C., Holzenberger, M., Mladenovic, Z., Salvat, C., Pecchi, E., Berenbaum, F., et al. Pro-inflammatory actions of visfatin/Nampt involve regulation of insulin signaling pathway and Nampt enzymatic activity. J Biol Chem 287, 15100, 2012.

39. Miyamoto, M., Ito, H., Mukai, S., Kobayashi, T., Yamamoto, H., Kobayashi, M., et al. Simultaneous stimulation of EP2 and EP4 is essential to the effect of prostaglandin E2 in chondrocyte differentiation. Osteoarthritis Cartilage 11, 644, 2003.

40. Ravichandran, K.S. Signaling via Shc family adapter proteins. Oncogene 20, 6322, 2001.
41. Davis, R.J. Transcriptional regulation by MAP kinases. Mol Reprod Dev 42, 459, 1995.

42. Kaoud, T.S., Devkota, A.K., Harris, R., Rana, M.S., Abramczyk, O., Warthaka, M., et al. Activated ERK2 is a monomer in vitro with or without divalent cations and when complexed to the cytoplasmic scaffold PEA-15. Biochemistry 50, 4568, 2011.

43. van der Heide, L.P., Hoekman, M.F., Biessels, G.J., and Gispen, W.H. Insulin inhibits extracellular regulated kinase $1 / 2$ phosphorylation in a phosphatidylinositol 3-kinase (PI3) kinase-dependent manner in Neuro2a cells. J Neurochem $\mathbf{8 6}$, 86, 2003.

44. Kim, M.O., Kim, S.H., Cho, Y.Y., Nadas, J., Jeong, C.H., Yao, K., et al. ERK1 and ERK2 regulate embryonic stem cell self-renewal through phosphorylation of Klf4. Nat Struct Mol Biol 19, 283, 2012.

45. Seymour, P.A., Freude, K.K., Dubois, C.L., Shih, H.P., Patel, N.A., and Sander, M. A dosage-dependent requirement for Sox 9 in pancreatic endocrine cell formation. Dev Biol 323, 19, 2008.

Address correspondence to: Rita A. Kandel, MD

Department of Pathology and Laboratory Medicine Mount Sinai Hospital 600 University Avenue Toronto M5G $1 X 5$

Canada

E-mail: rkandel@mtsinai.on.ca

Received: September 10, 2013 Accepted: January 31, 2014 Online Publication Date: April 30, 2014 


\section{This article has been cited by:}

1. Dr. Rita Kandel, Mr. Drew W Taylor, Dr. Nazish Ahmed, Dr. Gregory P Lunstrum, Dr. Allan E Gross, Dr. Eleftherios Diamandis. Collagen type XII and versican are present in the early stages of cartilage tissue formation by both redifferentating passaged and primary chondrocytes. Tissue Engineering Part A 0:ja. . [Abstract] [Full Text PDF] [Full Text PDF with Links]

2. Taylor Drew W., Ahmed Nazish, Parreno Justin, Lunstrum Gregory P., Gross Allan E., Diamandis Eleftherios P., Kandel Rita A.. Collagen Type XII and Versican Are Present in the Early Stages of Cartilage Tissue Formation by Both Redifferentating Passaged and Primary Chondrocytes. Tissue Engineering Part A, ahead of print. [Abstract] [Full Text HTML] [Full Text PDF] [Full Text PDF with Links] [Supplemental Material]

3. Justin Parreno, Sneha Raju, Mortah Nabavi Niaki, Katarina Andrejevic, Amy Jiang, Elizabeth Delve, Rita Kandel. 2014. Expression of type I collagen and tenascin $\mathrm{C}$ is regulated by actin polymerization through MRTF in dedifferentiated chondrocytes. FEBS Letters 588, 3677-3684. [CrossRef] 


\title{
Collagen Type XII and Versican Are Present in the Early Stages of Cartilage Tissue Formation by Both Redifferentating Passaged and Primary Chondrocytes
}

\author{
Drew W. Taylor, PhD, ${ }^{1-3}$ Nazish Ahmed, PhD, ${ }^{1,2}$ Justin Parreno, MSc, ${ }^{2}$ Gregory P. Lunstrum, PhD, ${ }^{4}$ \\ Allan E. Gross, MD, ${ }^{2,3}$ Eleftherios P. Diamandis, MD, and Rita A. Kandel, MD ${ }^{1-3}$
}

Current approaches to cartilage tissue engineering require a large number of chondrocytes. Although chondrocyte numbers can be expanded in monolayer culture, the cells dedifferentiate and unless they can be redifferentiated are not optimal to use for cartilage repair. We took advantage of the differential effect of culture conditions on the ability of passaged and primary chondrocytes to form cartilage tissue to dissect out the extracellular matrix (ECM) molecules produced and accumulated in the early stages of passaged cell cartilage tissue formation as we hypothesized that passaged bovine cells that form cartilage accumulate a pericellular matrix that differs from cells that do not form cartilage. Twice passaged bovine chondrocytes (P2) (cartilage forming), or as a control primary chondrocytes (P0) (which do not generate cartilage), were cultured on three-dimensional membrane inserts in serum-free media. P2 redifferentiation was occurring during the first 8 days as indicated by increased expression of the chondrogenic genes Sox9, collagen type II, aggrecan, and COMP, suggesting that this is an appropriate time period to examine the ECM. Mass spectrometry showed that the P2 secretome (molecules released into the media) at 1 week had higher levels of collagen types I, III, and XII, and versican while type II collagen and COMP were found at higher levels in the P0 secretome. There was increased collagen synthesis and retention by P2 cells compared to P0 cells as early as 3 days of culture. Confocal microscopy showed that types XII, III, and II collagen, aggrecan, versican, and decorin were present in the ECM of P2 cells. In contrast, collagen types I, II, and III, aggrecan, and decorin were present in the ECM of P0 cells. As primary chondrocytes grown in serumcontaining media, a condition that allows for the generation of cartilage tissue in vitro, also accumulate versican and collagen XII, this study suggests that these molecules may be necessary to provide a microenvironment that supports hyaline cartilage formation. Further study is required to determine if these molecules are also accumulated by passaged human chondrocytes and their role in promoting hyaline cartilage formation.

\section{Introduction}

A RTICULAR CARTILAGE IS AN avascular load-bearing connective tissue covering the articulating ends of bones in synovial joints that absorbs and distributes forces while permitting low-friction articulation. This functionality is dependent on the composition and proper organization of the matrix molecules that comprise the tissue. ${ }^{1}$ While the most abundant collagen of articular cartilage is collagen II, other types are also present to a lesser extent. Extensive cross-linking of the various collagen types is vital to tissue material strength, ${ }^{2}$ and the type and amount of collagens are likely to play an important role in the ability to trap other extracellular matrix (ECM) macromolecules. Proteoglycans, the second largest constituent of ECM, include aggrecan, which forms large aggregates distributed within the collagen network, and provides a high fixed charge, entrapping water and allowing the tissue to withstand compression. Other smaller leucine-rich proteoglycans, including biglycan and decorin, are also found in the ECM. Despite their relative low abundance, knockout studies have indicated that they play a crucial role in providing matrix stability. ${ }^{2-4}$

\footnotetext{
${ }^{1}$ BioEngineering of Skeletal Tissues Team, CIHR, Ottawa, Ontario, Canada.

${ }^{2}$ Department of Pathology and Laboratory Medicine and Lunenfeld Tanenbaum Research Institute, Mount Sinai Hospital, University of Toronto, Toronto, Ontario, Canada.

${ }^{3}$ Institute of Biomaterials and Biomedical Engineering, University of Toronto, Toronto, Ontario, Canada.

${ }^{4}$ Department of Research, Shriners Hospital for Children, Portland, Oregon.
} 
Noncollagenous proteins, including COMP and matrilins, play roles in matrix assembly, maintenance, and metabolism. ${ }^{5-7}$ Thus, the composition of articular cartilage is very complex.

Once damaged, articular cartilage is unable to repair, which has stimulated an increasing interest in developing cell and tissue-based repair/regeneration therapies. ${ }^{8-15}$ However, a large number of chondrocytes are required for these approaches and only a small number of cells can be harvested from an individual. Increasing the cell number by passaging chondrocytes in monolayer culture leads to loss of the chondrocyte phenotype and an inability to form cartilage tissue. ${ }^{16} \mathrm{~A}$ number of approaches have been developed to redifferentiate passaged chondrocytes, including threedimensional (3D) culture in pellets, gels, filters, or scaffolds in the presence or absence of growth factors, ${ }^{17,18}$ monolayer expansion with growth factors, ${ }^{19}$ or directly adding factors into the culture medium to enhance redifferentiation. ${ }^{20,21}$ However, studies have shown that the phenotype was not fully restored (or maintained). ${ }^{17,22}$ Identifying conditions that favor formation of articular cartilage of appropriate composition and organization would be a significant advance in cartilage tissue engineering.

We recently described a method by which passaged chondrocytes can be induced to redifferentiate and accumulate ECM rich in proteoglycans and type II collagen in vitro when cultured on type II collagen-coated membrane inserts (3D) in defined serum-free growth factor-free chondrogenic medium $(\mathrm{SF}){ }^{23}$ In contrast, culture of primary chondrocytes under these SF conditions did not support formation of hyaline-like cartilage, as these cells required the presence of serum to accumulate ECM. ${ }^{23}$ As the presence or absence of serum in the media of 3D cultures can affect whether $\mathrm{P} 0$ and $\mathrm{P} 2$ cells accumulate hyaline matrix molecules, we took advantage of the differential effect of these culture conditions to dissect out the pericellular matrix molecules produced and accumulated in the early stages of passaged cell redifferentiation and tissue formation. We hypothesized that passaged bovine cells that form cartilage accumulate a pericellular matrix that differs from cells that do not form cartilage. Identification of these macromolecules will not only facilitate study of which matrix molecules are important for cartilage formation but also may then serve as markers that can be used to identify culture conditions and/or scaffolds that will favor articular cartilage formation by passaged chondrocytes and facilitate rapid screening of culture conditions for chondrogenic-inducing potential.

\section{Materials and Methods}

\section{Cell culture}

Articular cartilage was harvested from bovine metacarpophalangeal joints (6-9 months old) as described previously. ${ }^{24}$ Chondrocytes were isolated from cartilage of an individual animal by enzymatic digestion with $0.1 \%$ collagenase A (Roche Diagnostics GmbH, Mannheim, Germany) for $18 \mathrm{~h}$ at $37^{\circ} \mathrm{C}$. Cells were either cryopreserved in liquid nitrogen for later use or resuspended in HAMS F12 containing 5\% fetal bovine serum (FBS; HyClone, Logan, UT), seeded in monolayer at a density of 2000 cells $/ \mathrm{cm}^{2}$, and grown under standard culture conditions. When cells reached $\sim 80 \%$ confluence, they were passaged and replated at the same seeding density. Bovine P2 $\left(2.0 \times 10^{6}\right.$ cells/membrane $)$ and P0 cells $\left(2.0 \times 10^{6}\right.$ cells/membrane $)$ were separately suspended in serum-free media (SF) consisting of high glucose Dulbecco's modified Eagle's medium, $\mathrm{ITS}^{+}(10 \mu \mathrm{g} /$ $\mathrm{mL}$ insulin, $0.5 \mu \mathrm{g} / \mathrm{mL}$ transferrin, $0.67 \mathrm{ng} / \mathrm{mL}$ selenium, $5.35 \mu \mathrm{g} / \mathrm{mL}$ linoleic acid, and $1.25 \mathrm{mg} / \mathrm{mL}$ bovine serum albumin; BD Bioscience, Billerica, MA), proline $(40 \mu \mathrm{g} /$ $\mathrm{mL})$, pyruvate $(110 \mu \mathrm{g} / \mathrm{mL})$, dexamethasone $(0.1 \mu \mathrm{M})$, and ascorbate-2-phosphate $(50 \mu \mathrm{g} / \mathrm{mL})$. Cells were seeded onto type II collagen-coated membrane plate inserts $\left(60 \mathrm{~mm}^{2}\right.$, Millicell ${ }^{\circledR}$; Millipore Co., Bedford, MA) prepared as described previously. ${ }^{25}$ Selected cultures were grown under the same culture conditions except that the media were supplemented with $20 \%$ FBS (serum). The cells were grown for up to 3 weeks, media were changed every 2 days, and collected at day $1,3,5$, and 7 of culture. The media was centrifuged to remove any cells in suspension and stored at $-80^{\circ} \mathrm{C}$ until further analysis (Supplementary Fig. S1; Supplementary Data are available online at www.liebertpub .com/tea).

\section{Protein extraction and sample preparation for mass spectrometry}

Chondrocytes (P2 and P0) were grown in media supplemented with insulin rather than $\mathrm{ITS}^{+}$to eliminate the bovine serum albumin present in ITS $^{+}$, which could affect analysis. Media were changed every 2 days, collected separately, and protein content quantified by the Bradford protein assay reagent (Pierce Biotechnology, Rockford, IL). Media collected within the first week were combined for mass spectrometry analysis.

To remove excess salts, samples were dialyzed in a molecular porous membrane tubing (Spectra/Por Dialysis Membrane; Spectrum Laboratories, Inc., Rancho Dominguez, CA) against an ammonium bicarbonate buffer $(50 \mathrm{mM})$ overnight, frozen at $-80^{\circ} \mathrm{C}$, lyophilized, then denatured with $8 \mathrm{M}$ urea (in $\mathrm{H}_{2} \mathrm{O}$ ), and reduced with dithiothreitol (final concentration, $13 \mathrm{mM}$; Sigma-Aldrich Ltd., St. Louis, MO). Samples were then alkylated with iodoacetamide (final concentration, $125 \mathrm{mM}$; Sigma-Aldrich Ltd.) and desalted using a NAP5 column (GE Healthcare, Buckinghamshire, United Kingdom) as per the manufacturer's instructions. Samples were lyophilized and digested in trypsin (trypsin/ protein ratio $1: 50 ; 120 \mu \mathrm{L} 50 \mathrm{mM}$ ammonium bicarbonate, $100 \mu \mathrm{L}$ methanol, $150 \mu \mathrm{L}$ water) overnight at $37^{\circ} \mathrm{C}$. Samples were loaded onto a PolySULFOETHYL ATM column (The Nest Group, Southborough, MA), containing a hydrophilic anionic polymer (poly-2-sulfoethyl aspartamide, $200 \AA$ pore size, $5 \mu \mathrm{m}$ diameter). Fractionation was performed using an Agilent $1100 \mathrm{HPLC}$ system $(200 \mu \mathrm{L} / \mathrm{min}$ flow rate $)$. A linear gradient of mobile phase B $(0.26 \mathrm{M}$ formic acid in $10 \%$ acetonitrile and $1 \mathrm{M}$ ammonium formate) was applied and eluate was monitored at $280 \mathrm{~nm}$.

\section{Mass spectrometry}

Peptides in fractions were microextracted using the ZipTip C18 pipette tip (Millipore) and eluted in $5 \mu \mathrm{L}$ of buffer $(64.5 \%$ acetonitrile, $35.4 \%$ water, $0.1 \%$ formic acid, and $0.02 \%$ trifluoroacetic acid). Eighty microliters of buffer (95\% water, $0.1 \%$ formic acid, $5 \%$ acetonitrile, and $0.02 \%$ trifluoroacetic acid) was added to each sample and $40 \mu \mathrm{L}$ was loaded using a 96-well microplate autosampler onto a 
2-cm C18 trap column, packed with Varian Pursuit $(5 \mu \mathrm{m}$ C18), using the EASY-nLC system (Proxeon Biosystems, West Palm Beach, FL) and running buffer A (0.1\% formic acid in water). Peptides were eluted from the trap column onto a resolving $5-\mathrm{cm}$ analytical $\mathrm{C} 18$ column packed with Varian Pursuit $(3 \mu \mathrm{m} \mathrm{C} 18)$ with an 8 - $\mu \mathrm{m}$ tip (New Objective, Woburn, MA) and an increasing concentration of buffer B ( $0.1 \%$ formic acid in acetonitrile). This LC setup was coupled online to an LTQ-Orbitrap XL (Thermo Fisher Scientific, Waltham, MA) mass spectrometer with a nanoelectrospray ionization source (Proxeon Biosystems). Fractions underwent a 54-min gradient (buffer A to buffer B), and eluted peptides were subjected to one full MS scan $(450-1450 \mathrm{~m} / \mathrm{z})$ in the Orbitrap at 60,000 resolution, followed by top 6 data-dependent MS/MS scans in the linear ion trap (LTQ Orbitrap). Using a charge state screening and preview mode, unassigned charge states as well as charges $1+$ and $4+$ were ignored.

\section{Protein data analysis}

Data files were created by the use of Mascot Daemon (version 2.2.0) and extract_msn. The resulting mass spectra were analyzed using Mascot (version 2.2; Matrix Science, Boston, MA) and X!Tandem (Global Proteome Machine Manager, version 2006.06.01) search engines on the nonredundant International Protein Index human database (version 3.46, 144,158 protein sequences). Resulting Mascot and X!Tandem search result files were loaded into Scaffold (version 2.0, Proteome Software) to cross-validate Mascot and X!Tandem data files. The protein false-positive rate was set to $1 \%$ by adjusting Mascot and X!Tandem thresholds within Scaffold. False-positive rates were calculated as the number of proteins identified by searching the reverse sequences (x2) divided by the total number of identified proteins. Normalized spectral counting was performed using Scaffold. Spectrum reports were uploaded into an in-house program for additional data analysis comparing proteins found between secretomes. Identified proteins that did not show a significant difference (determined by Student's $t$-test) between groups or that had $<10$ spectral counts between groups were eliminated.

\section{Evaluation of in vitro formed cartilage tissues}

At 3 weeks, cultures were harvested for histological evaluation. Tissues were fixed in $10 \%$ buffered formalin and embedded in paraffin. Five-micrometer sections were cut and stained with toluidine blue or hematoxylin and eosin. For immunohistochemical staining, sections were deparaffinized and digested with $0.4 \%$ pepsin (w/v) (Sigma-Aldrich Ltd.), blocked with $20 \%$ goat serum (v/v) (Sigma-Aldrich Ltd.), and then incubated overnight at $4^{\circ} \mathrm{C}$ with an antibody reactive with either type I collagen (1:100, T59103R; Meridian Life Science, Inc., Saco, ME) or type II collagen (1:100, MAB8887; Millipore), followed by incubation with the Alexa-488 goat anti-rabbit (1:300; Invitrogen, Paisley, United Kingdom) or Alexa-594 goat anti-mouse secondary antibody. Nuclei were stained with DAPI $(1: 10,000$; Invitrogen) and sections coverslipped (Permafluor). Images were collected at a single representative focal plane (resolution $1024 \times 1024$ pixels) using a $60 \times$ objective (Nikon Eclipse C1si; Nikon Corporation, Tokyo, Japan).
Matrix accumulation was quantified biochemically following harvest of the tissues at 3 weeks and digestion by papain $(40 \mu \mathrm{g} / \mathrm{mL}$ in $20 \mathrm{mM}$ ammonium acetate, $1 \mathrm{mM}$ EDTA, and $2 \mathrm{mM}$ DTT; Sigma-Aldrich Ltd.) for $48 \mathrm{~h}$ at $65^{\circ} \mathrm{C}$, as described previously. ${ }^{25}$ Sulfated glycosaminoglycans were quantified using the dimethylmethylene blue dye-binding assay (Polysciences, Inc., Washington, PA) and spectrophotometry (wavelength: $525 \mathrm{~nm}$ ). ${ }^{25}$ DNA content was quantified using the Hoechst dye 33258 assay (Polysciences, Inc.) and fluorometry (excitation wavelength: $365 \mathrm{~nm}$ and emission wavelength: $458 \mathrm{~nm}){ }^{26}$

\section{RNA extraction and quantitative $P C R$}

Total RNA was isolated using Trizol ${ }^{\circledR}$ (Gibco BRL, Rockville, MD). Invitrogen Superscript II reverse transcription kit was used to reverse transcribe $0.5 \mu \mathrm{g}$ of total RNA according to the manufacturer's instructions (Invitrogen, Carlsbad, CA). Each reaction contained 40 units $/ \mathrm{mL}$ of recombinant ribonuclease inhibitor RNase OUT ${ }^{\mathrm{TM}}$ (Invitrogen), $50 \mu \mathrm{g} / \mathrm{mL}$ of random hexamers, $10 \mathrm{mM}$ dNTPs, and 200 units of SuperScript II enzyme. Gene expression was determined using sequence-specific primers (Supplementary Table S1), SYBR green dye I, and Realplex2 Master Cycler (Eppendorf, Hamburg, Germany) (real time polymerase chain reaction, qPCR) according to the manufacturer's instructions, and data analyzed with Mastercycler EP Realplex. One microliter cDNA was amplified in $20 \mu \mathrm{L}$ final volume with $0.2 \mu \mathrm{M}$ each primer suspended in QuantiFast SYBR Green Master Mix (Qiagen, Hilden, Germany). Amplification parameters were identical for each primer pair; after the initial $10 \mathrm{~min}$ at $95^{\circ} \mathrm{C}$ to activate the enzyme, $15 \mathrm{~s}$ denaturation at $95^{\circ} \mathrm{C}$ was followed by $30 \mathrm{~s}$ annealing at $60^{\circ} \mathrm{C}$, and amplification data were collected for up to 40 cycles. Mean relative quantification values were calculated with the $\Delta \Delta \mathrm{Ct}$ method, using $18 \mathrm{~S}$ rRNA as endogenous control and primary bovine chondrocytes as a calibrator.

\section{Quantification of proteoglycan and collagen synthesis}

After 3 and 8 days, chondrocyte cultures were incubated in the presence of both ${ }^{35} \mathrm{~S} \mathrm{SO}_{4}(1 \mu \mathrm{Ci} /$ culture; PerkinElmer, Mississauga, Canada) to label proteoglycans and $\left[{ }^{3} \mathrm{H}\right]$ proline $(1 \mu \mathrm{Ci} /$ culture; PerkinElmer $)$ to label collagen for $48 \mathrm{~h}$. In chondrocyte cultures, 90\% of proline becomes incorporated into collagen. The medium was collected and divided in two parts, proteoglycans were precipitated by adding 100\% cold ethanol (3:1 ratio) and collagen was precipitated by adding $70 \%$ ammonium sulfate solution (3:4 ratio) overnight at $4^{\circ} \mathrm{C}$. Samples were each centrifuged at $14,000 \mathrm{rpm}$ for $30 \mathrm{~min}$ at $4^{\circ} \mathrm{C}$, washed in $70 \%$ ethanol, and resuspended in either $4 \mathrm{M}$ guanidine hydrochloride to solubilize the proteoglycans or $10 \%$ sodium dodecyl sulfate in Tris buffer $(0.1 \mathrm{M}, \mathrm{pH} 7.0)$ to solubilize collagen. To quantify newly synthesized collagen and proteoglycans accumulated in the tissue, cultures were washed thrice in phosphate-buffered saline (PBS) and digested by papain. Radioisotope incorporation in media and tissue was determined using a $\beta$-liquid scintillation counter (Beckman LS6000TA; Beckman Instruments, Mississauga, ON, Canada). The amount of synthesized molecules in each fraction (culture or media) as well as the total matrix synthesis were expressed relative to the DNA content. 
Immunohistochemistry and confocal microscopy of early cultures

To assess ECM accumulation at early time points, the cells were processed differently than the tissues. Cells at 1 and 3 days of culture were fixed in $4 \%$ paraformaldehyde at room temperature for $15 \mathrm{~min}$ and then permeabilized with $0.2 \%$ Triton-X in PBS for $15 \mathrm{~min}$. For staining of collagens, samples were sequentially digested with $2.5 \mathrm{mg} / \mathrm{mL}$ pepsin (P7012-1G, in PBS at $\mathrm{pH} 2$; Sigma-Aldrich Ltd.) for $10 \mathrm{~min}$ at $37^{\circ} \mathrm{C}, 2.5 \mathrm{mg} / \mathrm{mL}$ of trypsin (T7409 Tris-buffered saline; Sigma-Aldrich Ltd.) for $30 \mathrm{~min}$ at $37^{\circ} \mathrm{C}$, and $25 \mathrm{mg} / \mathrm{mL}$ hyaluronidase (H3506; Sigma-Aldrich Ltd.) for $30 \mathrm{~min}$ at $37^{\circ} \mathrm{C}$. For staining of proteoglycans, cells were digested with $0.25 \mu \mathrm{M}$ of chondroitinase $\mathrm{ABC}$ (in $0.1 \mathrm{M}$ Tris $\mathrm{pH} 8$, $0.05 \mathrm{M} \mathrm{NaOAc}$ ) for $1 \mathrm{~h}$ at $37^{\circ} \mathrm{C}$. Samples were blocked with $20 \%$ goat serum and $0.1 \%$ Triton-X in PBS for $1 \mathrm{~h}$ at $37^{\circ} \mathrm{C}$ and incubated with an antibody reactive to either collagen type I [1:300 in dilution buffer (DB: 10\% goat serum and $0.1 \%$ Triton-X in PBS); Meridian Life Science, Inc.], collagen type II (clone 6B3, 1:300 in DB; Labvision, Freemont, CA), collagen type III (ab7778, 1:100 in DB; Abcam, Toronto, Canada), collagen type XII (NC3 domain, clone 1851, rabbit polyclonal, 1:50 in DB), aggrecan (clone 6B4, $1: 100$ in DB; Abcam), versican (clone 12C5, 1:50 in DB; Developmental Studies Hybridoma Bank, Iowa City, IA), or decorin (sc-22753, 1:150 in DB; Santa Cruz, Dallas, TX) overnight at $4{ }^{\circ} \mathrm{C}$. Samples were washed in $0.2 \%$ Triton-X, and then incubated with the goat anti-rabbit IgG secondary antibody (1:500 in DB) conjugated with Cy5 (Jackson Immunoresearch Laboratories, West Grove, PA) or goat antimouse source of the primary antibody, at $25^{\circ} \mathrm{C}$ for $1 \mathrm{~h}$. After washing with $0.2 \%$ Triton-X in PBS, nuclei were counterstained with DAPI (1:10,000 in PBS; Invitrogen), washed in PBS, coverslipped (Permafluor Mountant solution, anti-fade; Thermo Scientific, Pittsburgh, PA), and stored in darkness at $4^{\circ} \mathrm{C}$ until examined by the Quorum WaveFX Spinning Disc Confocal System (Guelph, Canada) with optimized Yokogawa CSU X1, Hamamatsu EM-CCD digital camera Image EM (C9100-13), and Leica DMI6000B inverted research grade motorized microscope run by Velocity 5.2.2 Acquisition software (Improvision/PerkinElmer, Waltham, MA).

\section{Statistical analysis}

Three independent sets of experiments were performed for cell culture studies. All conditions were done in triplicate. Data are presented as mean \pm standard error of the mean. Results were analyzed using a one-way analysis of variance, and all pairwise comparisons between groups were conducted using the Tukey post hoc test. $p$-Values $\leq 0.05$ were considered to be statistically significant.

\section{Results}

\section{Redifferentiation of passaged chondrocytes}

P0 and P2 chondrocytes were cultured independently for up to 3 weeks to confirm their capacity to accumulate ECM and form cartilage tissue in serum-free 3D culture (SF) supplemented with either ITS $^{+}$or insulin only (Supplementary Fig. S2). P0 cells grown in serum-free conditions did not accumulate matrix. In contrast, $\mathrm{P} 2$ cells accumulated
ECM rich in proteoglycans as shown through a histological examination and as quantified biochemically. Immunostaining showed type II collagen and little type I collagen in the tissue. The tissue was more cellular compared to P0 cultures (Supplementary Fig. S2).

Passaged chondrocytes grown in media supplemented with insulin alone (condition used for mass spectroscopy studies) supported chondrocyte redifferentiation and hyaline cartilage tissue formation. The amount of proteoglycan accumulation by $\mathrm{P} 2$ cells was similar whether P2 cells were grown in $\mathrm{ITS}^{+}$or insulin only and had similar cellularity as determined by the DNA content (Supplementary Fig. S3). This suggests that insulin can replace ITS in supporting passaged cell redifferentiation.

\section{Differences in the secretome between $P 2$ and $P 0$ cells in the early stages of culture}

The secretome (cell-conditioned media) of P2 and P0 cells grown SF for 1 week of culture was analyzed by mass spectrometry (Fig. 1). One thousand two hundred sixty proteins were identified in the combined secretomes, 807 in P0, and 1122 in P2 cultures, with 669 that overlapped between groups (Fig. 1). Six thousand six hundred eightyeight peptides were identified in the combined secretomes, 3758 in P0, and 5882 in P2 cultures, with 2952 peptides that overlapped between groups. Spectral counts were log transformed, scaled within each replicate, and used to generate a two-dimensional heat map with hierarchical clustering using the R-Project open-source software (Fig. 1). One hundred seventy-one proteins with a spectral count difference of at least 10-fold were present at significantly different levels. Spectral counts of cartilage-associated proteins, including collagen types I, II, III, and XII, aggrecan, versican, biglycan, decorin, proteoglycan-4, COMP, and matrilin-3, are presented in Figure 2. Collagen types I, III, and XII, and versican were found at higher levels within the P2 secretome, while type II collagen and COMP were found at higher levels in the $\mathrm{P} 0$ secretome. Proteins of interest known to be present in cartilage ECM are presented along with number of peptide fragments detected, length of amino acid sequence, and percent sequence coverage of the peptides in Supplementary Table S2.

\section{Differential expression of genes between P2 and $P O$ cells over time}

To characterize gene expression of proteins of interest, mRNA was isolated at various time points within the first 2 weeks of culture and examined by qPCR (Fig. 3). Sox9 was initially lower in P2 cells until day 8 when it reached P0 levels, after which levels of sox 9 again dropped. Initially, expression of aggrecan and COMP was significantly lower in $\mathrm{P} 2$ cells but these increased, reaching levels expressed by $\mathrm{P} 0$ cells by 8 days. Expression of versican was not significantly different between $\mathrm{P} 0$ and $\mathrm{P} 2$ cells throughout the 2 weeks in culture. Type I collagen expression was initially 100-fold higher in P2 cells than P0, but dropped to about 10fold higher at 8 days. Expression of type II collagen in P2 cells increased steadily up to day 8 , where levels were then 10-fold higher than those found in P0 cells and remained significantly higher throughout 2 weeks. Type III collagen 


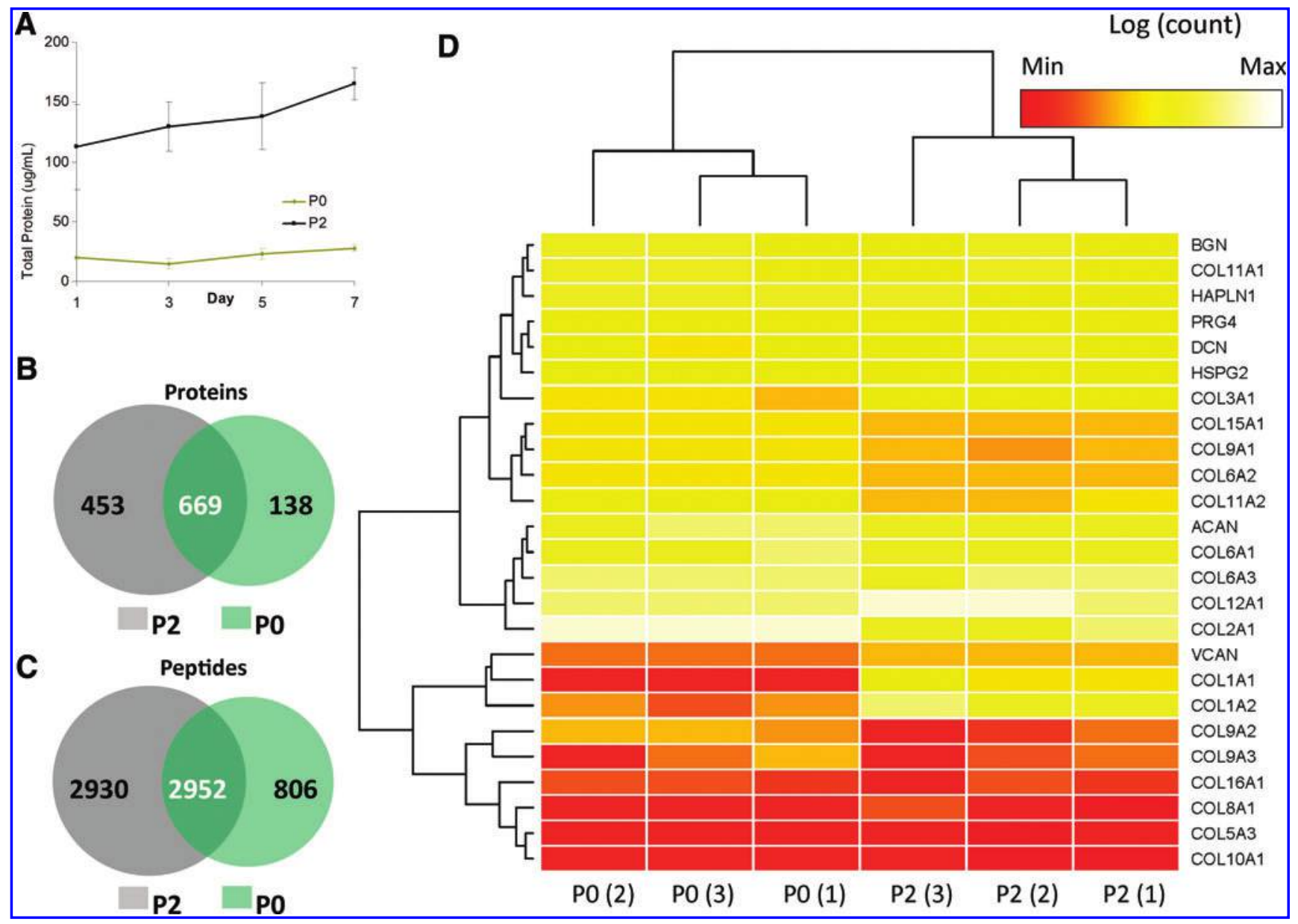

FIG. 1. Proteonomic analysis. Total protein present in the secretome in the first week of P2 and P0 culture (A). Venn diagrams of percentage of proteins $(\mathbf{B})$ and peptides $(\mathbf{C})$ in the secretome during the first week of P2 and P0 culture. Heat map of spectral counts for collagens and proteoglycans depict differences found in the secretome between P0 and P2 secretomes (D). Spectral counts were log transformed, scaled within each replicate, and used to generate a two-dimensional heatmap with hierarchical clustering. The colours indicate the levels of count with red representing the lowest level, followed by orange, then yellow and red as the highest level. Color images available online at www.liebertpub.com/tea

expression remained stable throughout 2 weeks in culture. Type XII collagen levels were significantly higher in P2 cells after just 2 days in culture and steadily deceased throughout 2 weeks although remaining higher than levels in P0.

\section{Collagens, not proteoglycans, are preferentially retained by $P 2$ cells compared to $P O$ cells in early cultures}

To examine retention of newly synthesized collagen and proteoglycans, the two major macromolecules in cartilage, the media and tissues were collected daily and those from days 1-3 to 8-10 were pooled to create two groups (Fig. 4). Within the first 3 days, proteoglycan synthesis was higher in P0 cells than P2 cells, but in both cultures, only small amounts were retained. By 8-10 days, proteoglycan synthesis for both cell types had decreased and was similar in amount. In contrast, P2 cells synthesized and retained significantly more collagens than P0 cells at both early (days 1-3) and later (days 8-10) time points. The percent of newly synthesized collagen retained in tissue formed by P0 cells decreased at the later time point.

\section{The ECM differs between cells}

\section{that will form cartilage tissue compared} to those that do not form cartilage tissue

Immunostaining and confocal microscopy were used to probe differences in ECM accumulation within the first 3 days of culture of the proteoglycans and collagens of interest identified by mass spectrometry (Fig. 5). Very little collagen type I was retained within the matrix produced by $\mathrm{P} 2$ versus $\mathrm{P} 0$ cultures, while collagen type III was retained in the ECM of both P0 and P2 at either time point. P0 cells accumulated small amounts of collagen type II, but little was seen in the ECM. Abundant collagen type II was accumulated in the matrix of P2 cultures by day 1 and this appeared to increase by day 3. Collagen type XII was only detected in the matrix accumulated by P2 cells and was present at day 1 . Versican was also absent from $\mathrm{P} 0$, but was present on day 1 in the P2 ECM and there appeared to be less staining by day 3. Aggrecan was seen in the P2 matrix on day 3 . Decorin was produced by $\mathrm{P} 0$ and $\mathrm{P} 2$ cells at both time points.

In previous studies, we showed the differential ability of P0 and P2 cells to form cartilage tissue when grown in the 


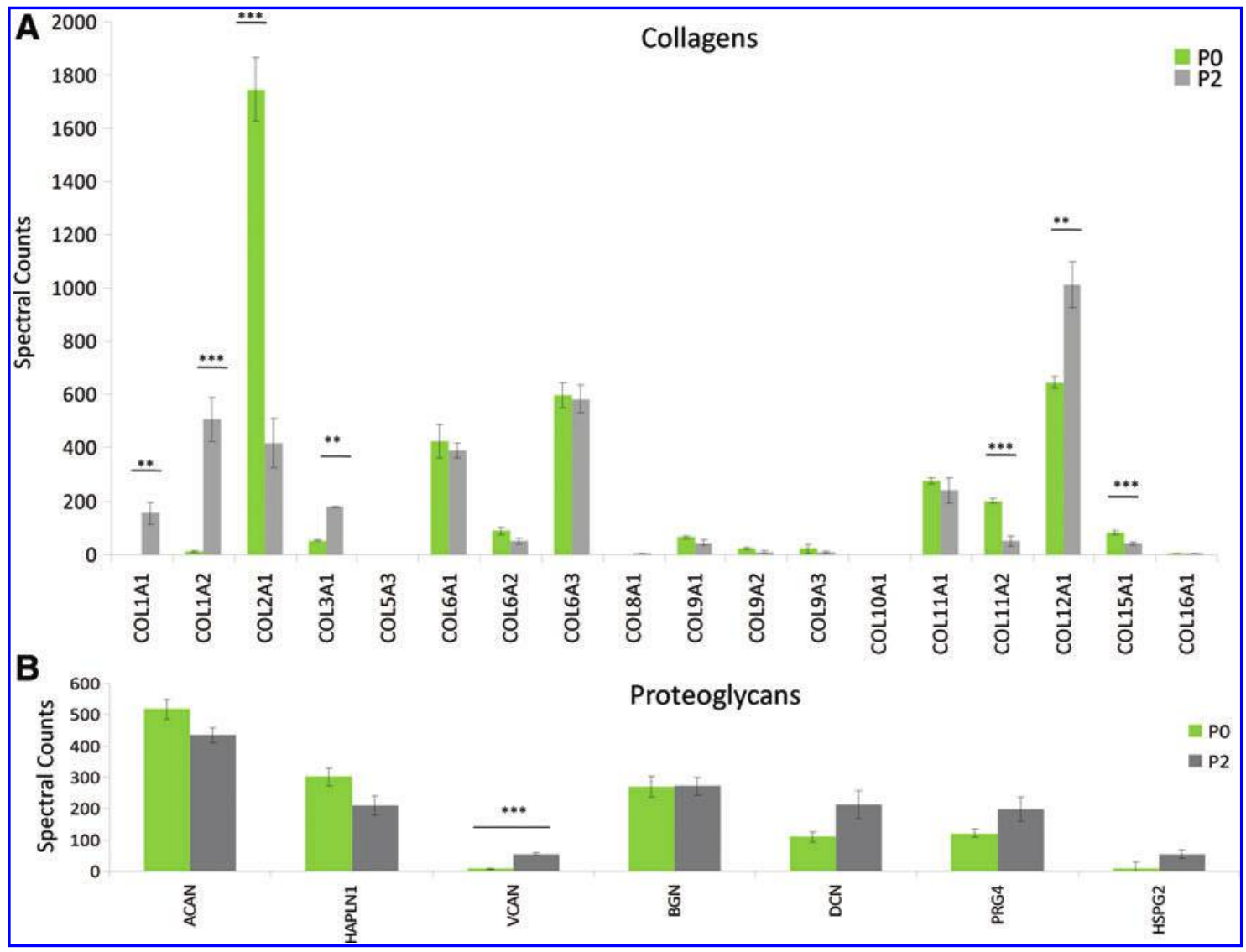

FIG. 2. Differences in $\mathrm{P} 2$ and $\mathrm{P} 0$ secretomes. Spectral counts determined through mass spectrometry for collagen (A) and proteoglycans (B). Significant differences between P0 and P2 culture media $* * p<0.01,{ }^{* * *} p<0.001$. Color images available online at www.liebertpub.com/tea

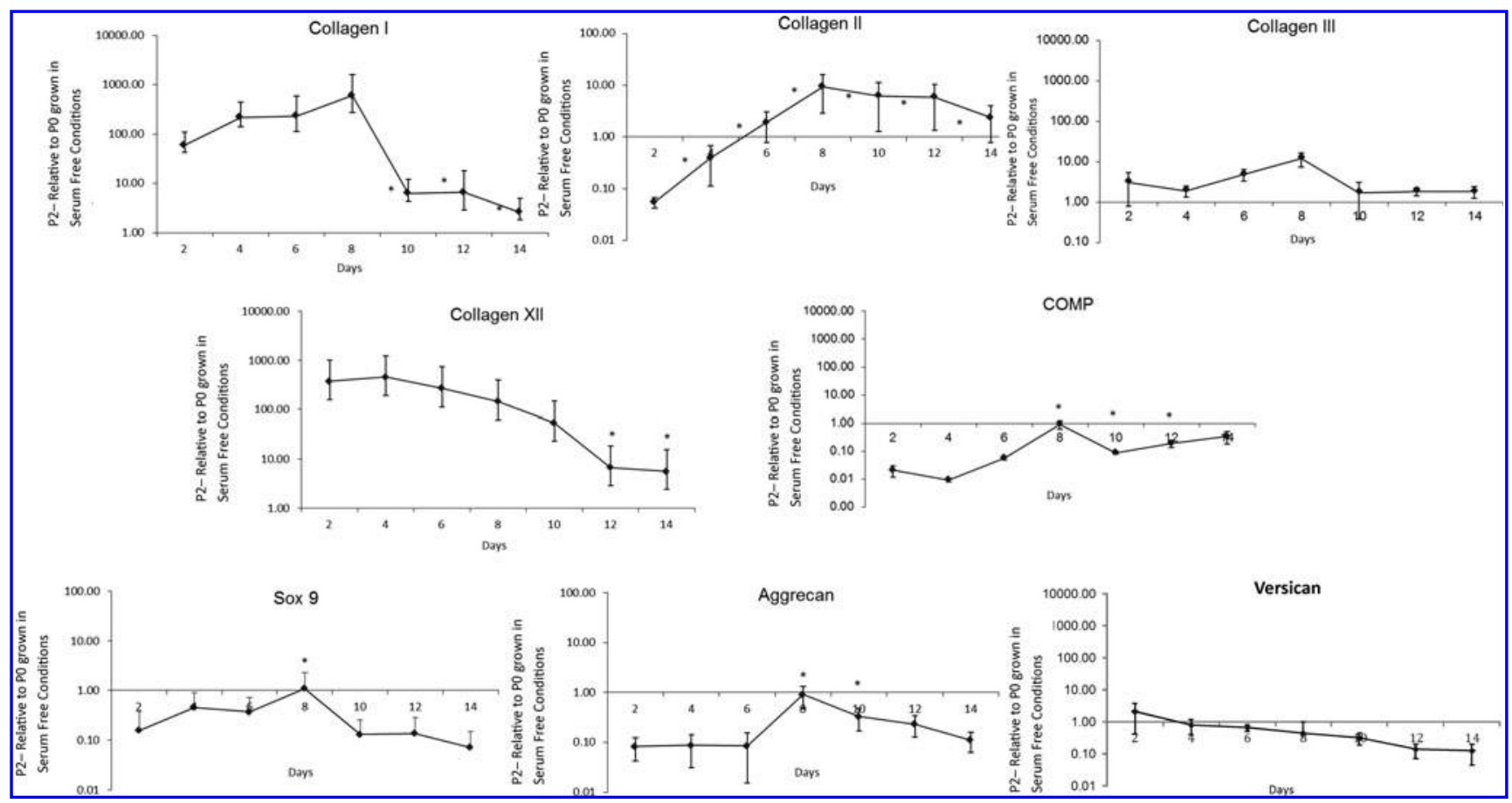

FIG. 3. Expression of genes by P2 and P0 cells over time. Gene expression was determined by real time PCR of molecules of interest, as identified by mass spectrometry. The results from three independent experiments were pooled $(n=9)$ and expressed as mean \pm standard error of the mean (SEM). All values are represented as P2 relative to P0 grown in serum-free conditions. * Significantly different from P2 at day $2(p<0.05)$. 


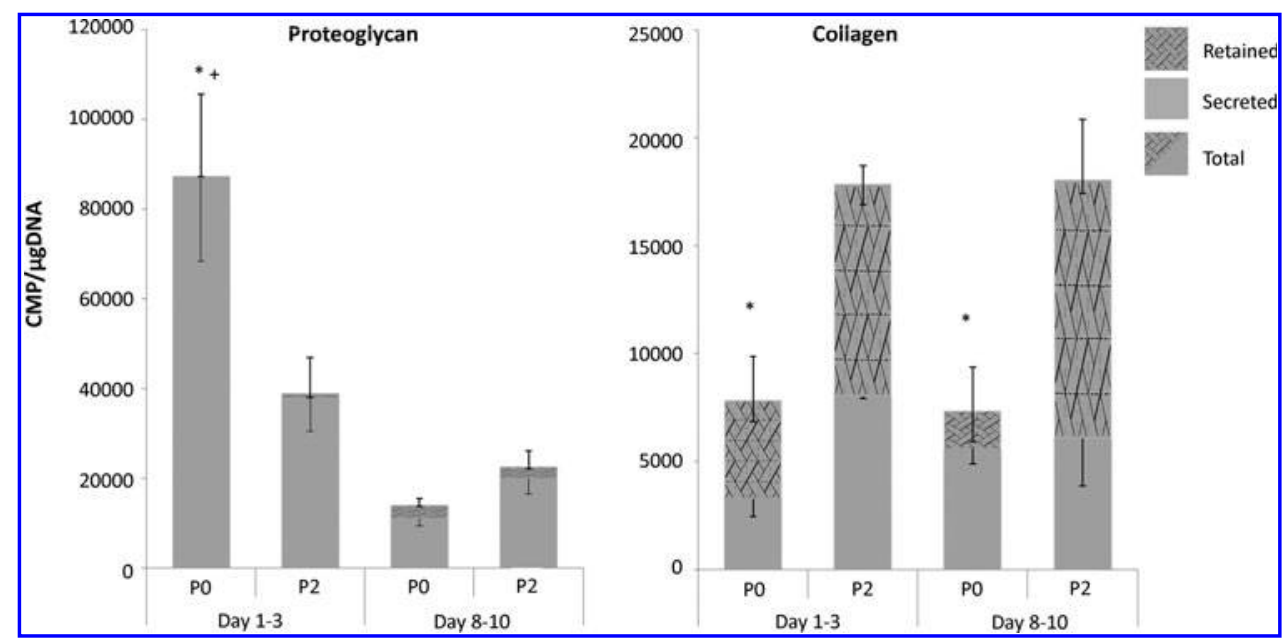

FIG. 4. Proteoglycan and collagen synthesis by primary and passaged cells in vitro. Total proteoglycan and collagen synthesis and retention were determined in P0 and P2 cultures between 1 and 3 days and 8 and 10 days in culture. One representative set is shown from a total of three independent experiments. Each experiment condition was performed in triplicate. Results are expressed as mean \pm SEM. * Total synthesis significantly different between P0 and P2 at the same time point, + total synthesis significantly different between time points of the same cell type $(p<0.5)$.

presence of serum. P0 cells will generate tissue, whereas P2 cells grown in the presence of FBS will not. ${ }^{23,27}$ To confirm the findings observed in SF cultures, we immunostained the cells cultured in serum-containing media at the same time points. P0 cells accumulated aggrecan, collagens type II and XII, and versican as early as 1 day in culture, whereas $\mathrm{P} 2$ cells grown in the presence of FBS did not accumulate collagen type XII or versican, similar to P0 cells grown SF (Fig. 6).

\section{Discussion}

To date, identifying a cell source, culture conditions, and/ or scaffold that allow for articular cartilage tissue engineering has been problematic. By taking advantage of in vitro culture systems that do or do not support hyaline cartilage tissue formation in vitro, it was possible to identify matrix molecules that were differentially expressed within the first 3 days of culture. Our study showed that versican, collagens type XII, II and III, decorin, and aggrecan were present in the matrix produced by cells that were going to form hyaline cartilage in vitro. As collagen types II and III, decorin, and aggrecan were present in small amounts in the cultures of passaged cells in serum-containing and primary cells in serum-free conditions (conditions that do not form tissue), suggests that their presence in the early time points (day 1) may not be sufficient to promote tissue formation. Collagen type II and aggrecan are clearly necessary for cartilage formation as they are the major components of articular cartilage and were observed to increase over time in chondrogenic cultures only. In contrast, type XII collagen and versican were absent from the ECM, under conditions in which cells were not destined to form cartilage tissue. Importantly, cells that form tissue whether grown in the presence or absence of serum both showed accumulation of these two molecules, supporting a role for them in early matrix accumulation. Further study will be required to confirm if these molecules are required for tissue formation. Interestingly, analysis of the secretome of redifferentiating passaged chondrocytes showed that these two molecules were secreted, suggesting that versican and type XII may be suitable for use to screen conditions in high-throughput assays to identify chondrogenic-promoting conditions.

The early time points (less than 10 days of culture) selected for this study were appropriate for three reasons. First, P2 redifferentiation appears to be occurring during the first week as the chondrogenic genes, collagen type II, aggrecan, sox 9 , and $C O M P$, were significantly upregulated by day 8 relative to day 2 of culture. Second, as early as 3 days of culture, a significant difference in collagen retention was detected between the cells that formed cartilage and those that did not. The data also showed that a greater number of unique proteins/peptides were detected in media from cultures that will form cartilage ( $\mathrm{P} 2$ in $\mathrm{SF}$ ) even up to 8 days than those that do not (P0 SF).

In keeping with our results, others have also shown a role for versican, a chondroitin sulfate proteoglycan, and collagen type XII in early cartilage tissue formation. ${ }^{27-34}$ Versican has been shown to be expressed during chondrogenesis, particularly in differentiating cells. Versican expression is localized in limb precartilage mesenchyme condensation, a precursor stage to chondrocyte differentiation. ${ }^{28,29}$ Shepard et al. have shown impaired joint morphogenesis and reduced limb length in chick versican knockdown studies. ${ }^{30}$ Versican overexpression, particularly the G1 portion or the V3 isoform, in chick wing buds led to enhanced chondrogenesis. ${ }^{35}$ In keeping with its role in chondrogenesis, versican gene expression levels rapidly decrease during growth and is expressed at low levels in mature articular cartilage. ${ }^{32}$ Versican may facilitate chondrogenesis by binding TGF $\beta$ in the matrix and regulating its signaling. ${ }^{30}$ Notably, the G3 domain of versican binds fibrillin, tenascin, and heparan sulfate proteoglycans and may be involved in retention of the cartilage ECM. ${ }^{36}$

Type XII collagen is a member of the FACIT collagen subfamily. ${ }^{37-39}$ Gregory et al. evaluated the distribution of collagen XII in rat cartilage from embryonic joint development through adulthood ${ }^{33}$ and showed that collagen XII was synthesized at the articular surface shortly after joint 
FIG. 5. P2 cells accumulate aggrecan, versican, and collagens type II and XII. P2 and P0 cells were grown in serum-free threedimensional culture for 1 or 3 days and then harvested and stained for various proteins. Immunoreactivity was detected using a fluorescent (either green or red) conjugated secondary antibody and confocal microscopy. Nuclei are stained with DAPI (blue). All images are taken at $100 \times$ magnification. Color images available online at www.liebertpub.com/tea
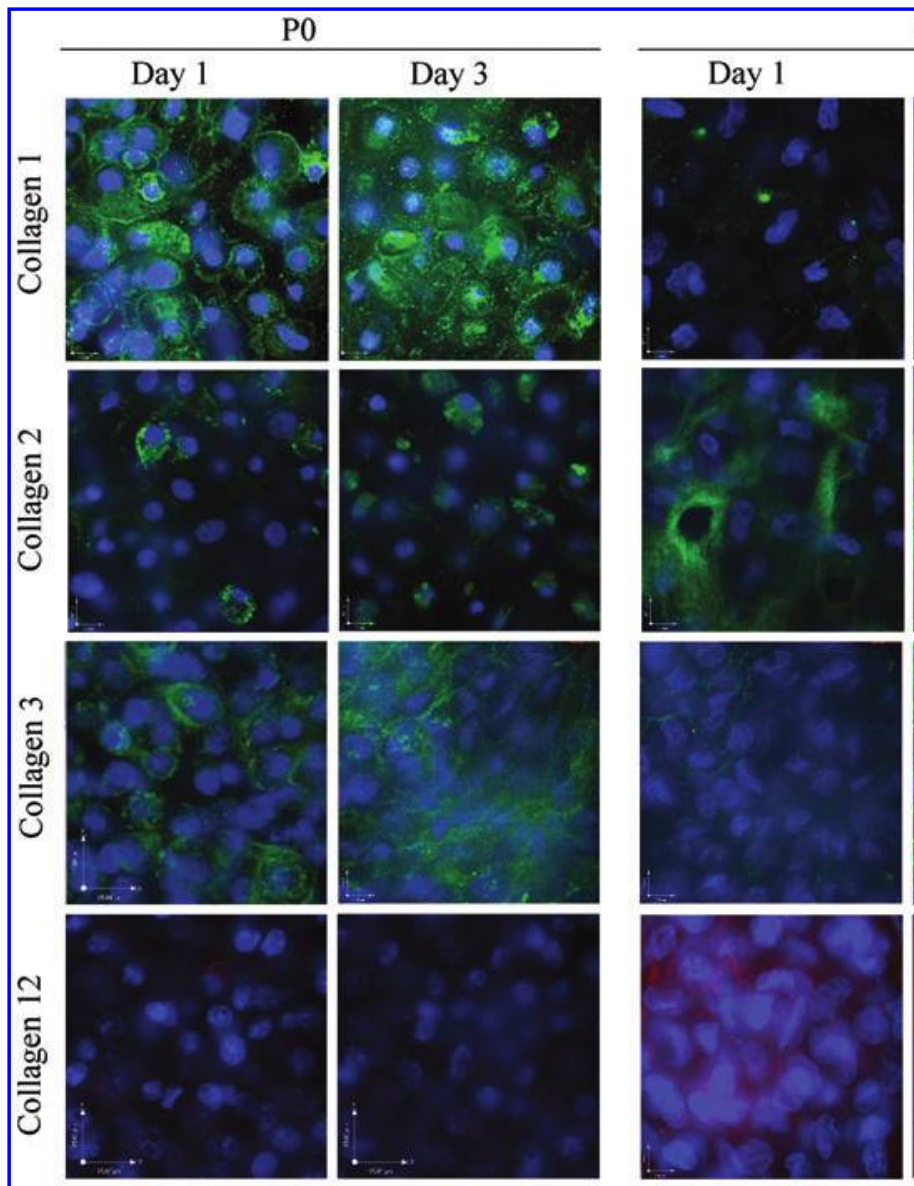

P2

Day 3
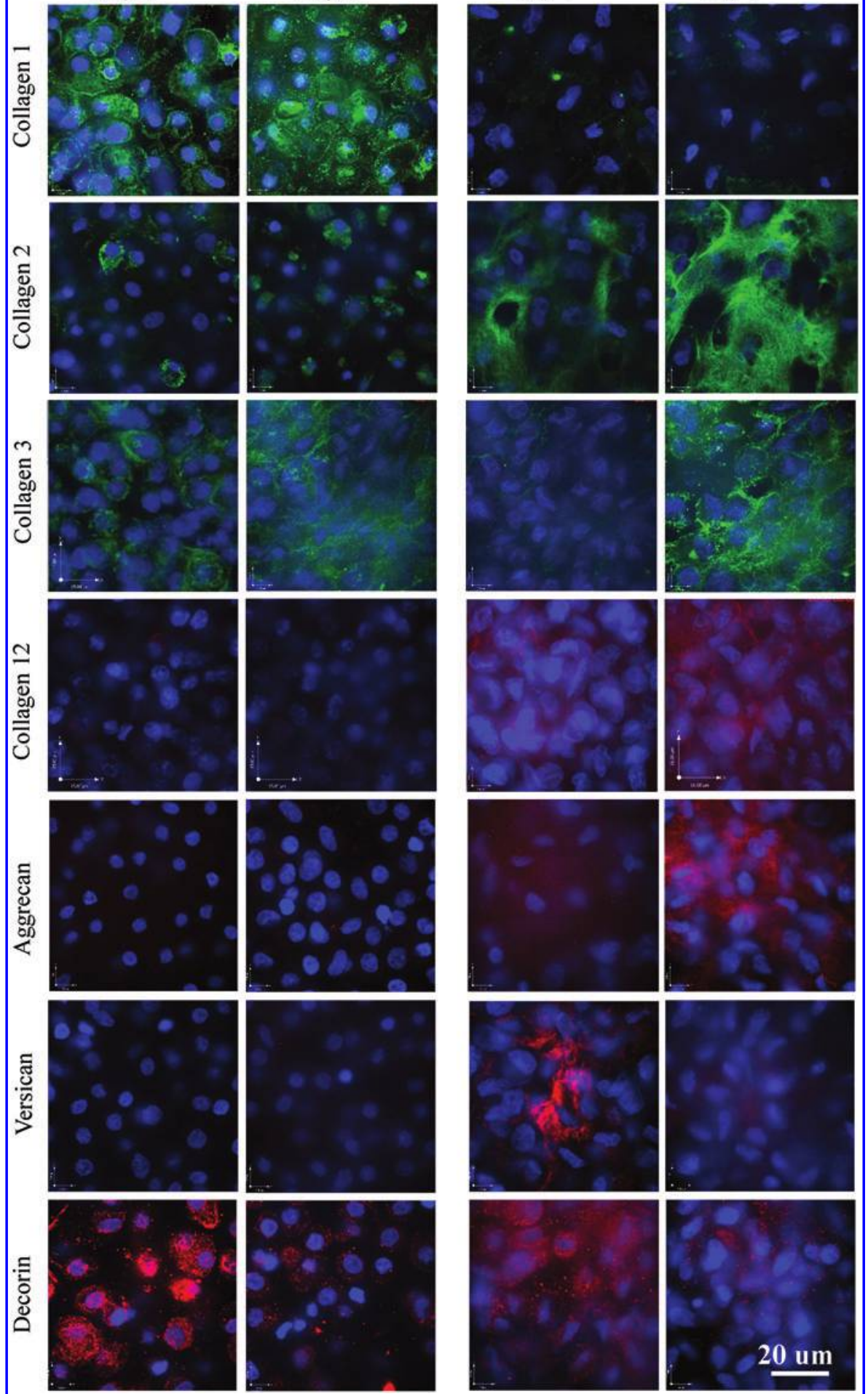

cavitation when an articular cartilage zone is first identified. Immunostaining detected collagen XII in the cartilage, which increased with age through day 28 . This collagen has also been detected in fetal bovine articular cartilage and perichondrium. ${ }^{34}$ Interestingly collagen type XII plays a role in regeneration of tissues other than cartilage. Wei et al. showed that type XII collagen colocalizes with tenascin and fibronectin in the regenerating newt limb, ${ }^{40}$ as gene expression was detected in the wound epithelium and mesenchyme as regeneration proceeded. Early contribution of collagen XII to the developing matrix is further supported by the fact that $\alpha 1[\mathrm{XII}]$ collagen gene was decreased by the mid-bud and late-bud blastema stages. ${ }^{40}$ How collagen type XII effects these changes is unknown, but influencing 


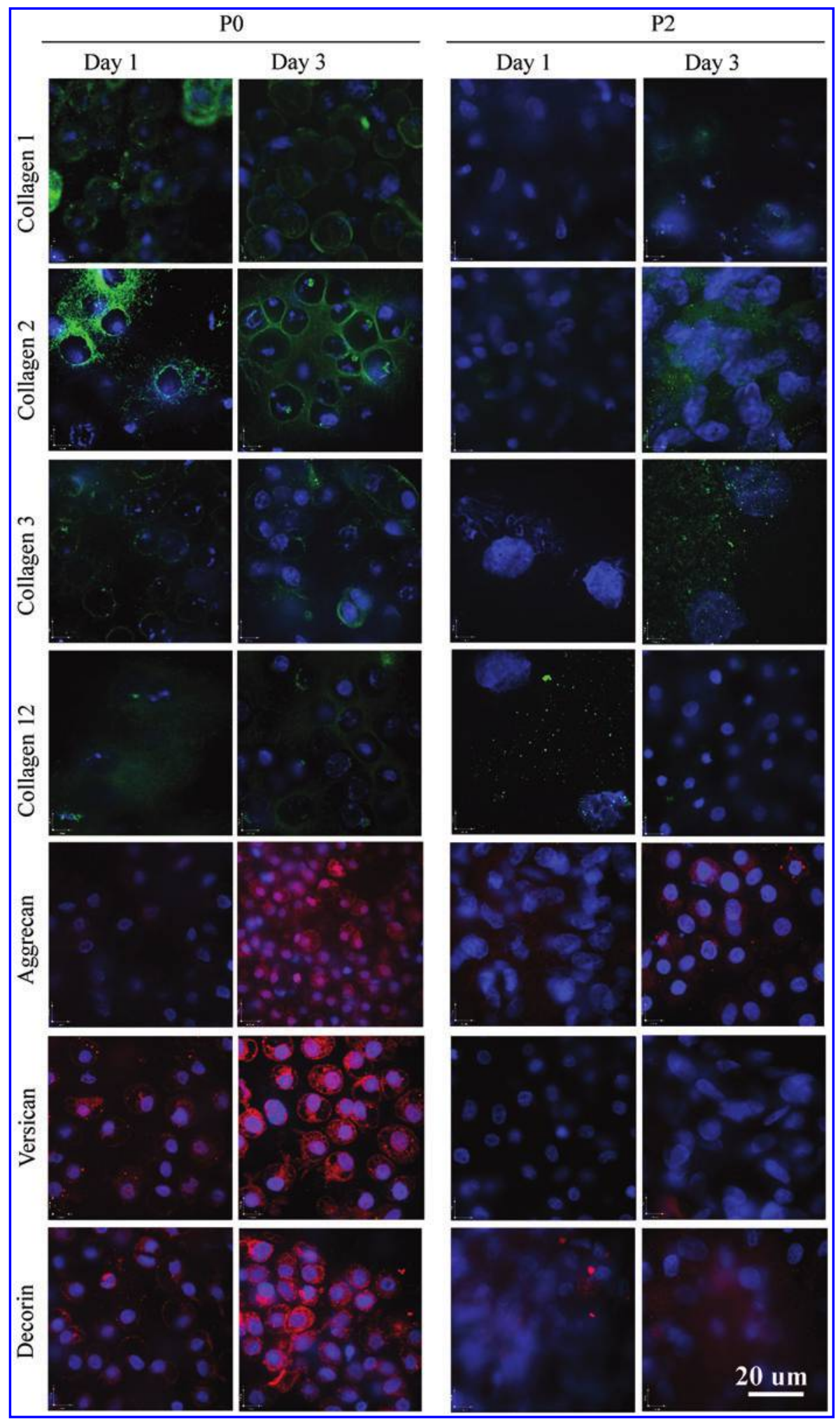

FIG. 6. P0 cells accumulate aggrecan, versican, and collagens type II and XII when grown in culture in media supplemented with $20 \%$ fetal bovine serum. P2 and P0 cells were grown in culture containing serum for 1 or 3 days and then harvested and stained for various proteins. Immunoreactivity was detected using a fluorescent (either green or red) conjugated secondary antibody and confocal microscopy. Nuclei are stained with DAPI (blue). All images are taken at $100 \times$ magnification. Color images available online at www .liebertpub.com/tea

collagen alignment ${ }^{33}$ or by binding to other molecules such as COMP, ${ }^{41}$ which are known to effect matrix organization and retention, is a possibility. Alternatively, collagen type XII may be influencing cell differentiation as knockout mice show delayed endothelial cell maturation. ${ }^{42}$ Polacek et al. have shown that type XII collagen is present in the secretome of human passaged chondrocytes. ${ }^{43}$ Interestingly, collagen type XII was not detected in cartilage explant secretome, suggesting a role for this collagen during redifferentiation and/or matrix accumulation. It is not known whether versican and type XII collagen interact in some way to influence matrix accumulation.

The presence of type I collagen, a marker of dedifferentiation, in the secretome of $\mathrm{P} 2$ cells was expected and may be due 
to the gradual change in the phenotype of $\mathrm{P} 2$ cells during the first week of culture. In support of this, type I collagen gene expression was stable during the first week of culture but decreased by over 100 -fold by day 10 . Similarly, mesenchymal stromal cells differentiate to chondrocytes slowly and have also been shown to require about $10-14$ days. ${ }^{44}$ Importantly, there was no evidence of retention of type I collagen within the nascent P2 ECM. Interestingly, levels appeared higher for chondrocyte-specific proteins in the $\mathrm{P} 0$ secretome, including collagen type II and COMP, under conditions in which the cells do not form cartilage. This suggests that these cells produce matrix molecules characteristic of cartilage but do not retain them in sufficient amounts to form tissue. The reason(s) for this requires further investigation.

It was not surprising that cells forming cartilage tissue have a different ECM as compared to cells that do not, as numerous studies have shown that the microenvironment as well as other factors may influence cell function. ${ }^{45-47}$ Our data do not determine if cells produce an ECM that favors accumulation of molecules such as collagen type XII and versican or whether cells grown in the right culture media produce these molecules that then favor cartilage tissue formation. However, our study does suggest that collagen XII and versican may serve as early biomarkers of the ability to form hyaline cartilage. This may be a way to assess the chondrogenic potential of cells grown under specific conditions and/or on scaffolds as these molecules are present in the secretome.

In summary, versican, collagens XII, III, and II, decorin, and aggrecan are early matrix molecules accumulated by passaged chondrocytes that will form cartilage tissue in vitro. However, versican and collagen type XII are accumulated only by cells that will form hyaline cartilage. Further study is required to determine if these molecules are also accumulated by passaged human chondrocytes and their role in promoting hyaline cartilage formation.

\section{Acknowledgments}

This work was made possible with the assistance of Ihor Batruch, Chris Smith, and Maria Pavlou of the Advanced Centre for Detection of Cancer Laboratory at Mount Sinai Hospital. We thank Mr. Harry Bojarski and Ryding-Reading Meat Packers, Toronto, for providing bovine tissues. This work was supported by funding from the Department of Defence W81XWH-10-1-0787.

\section{Disclosure Statement}

No competing financial interests exist.

\section{References}

1. Chen, C., Tambe, D.T., Deng, L., and Yang, L. Biomechanical properties and mechanobiology of the articular chondrocyte. Am J Physiol Cell Physiol 305, C1202, 2013.

2. McGann, M.E., Bonitsky, C.M., Ovaert, T.C., and Wagner, D.R. The effect of collagen crosslinking on the biphasic poroviscoelastic cartilage properties determined from a semi-automated microindentation protocol for stress relaxation. J Mech Behav Biomed Mater 34, 264, 2014.

3. Halper, J. Proteoglycans and diseases of soft tissues. Adv Exp Med Biol 802, 49, 2014.

4. Nikitovic, D., Aggelidakis, J., Young, M.F., Iozzo, R.V., Karamanos, N.K., and Tzanakakis, G.N. The biology of small leucine-rich proteoglycans in bone pathophysiology. J Biol Chem 287, 33926, 2012.

5. Budde, B., Blumbach, K., Ylostalo, J., Zaucke, F., Ehlen, H.W., Wagener, R., et al. Altered integration of matrilin-3 into cartilage extracellular matrix in the absence of collagen IX. Mol Cell Biol 25, 10465, 2005.

6. Wagener, R., Ehlen, H.W., Ko, Y.P., Kobbe, B., Mann, H.H., Sengle, G., et al. The matrilins-adaptor proteins in the extracellular matrix. FEBS Lett 579, 3323, 2005.

7. Halasz, K., Kassner, A., Morgelin, M., and Heinegard, D. COMP acts as a catalyst in collagen fibrillogenesis. $\mathrm{J}$ Biol Chem 282, 31166, 2007.

8. Matsuura, K., Utoh, R., Nagase, K., and Okano, T. Cell sheet approach for tissue engineering and regenerative medicine. J Control Release 190, 228, 2014.

9. Musumeci, G., Castrogiovanni, P., Leonardi, R., Trovato, F.M., Szychlinska, M.A., Di Giunta, A., et al. New perspectives for articular cartilage repair treatment through tissue engineering: a contemporary review. World J Orthop 5, 80, 2014.

10. Bhumiratana, S., Eton, R.E., Oungoulian, S.R., Wan, L.Q., Ateshian, G.A., Vunjak-and Novakovic, G. Large, stratified, and mechanically functional human cartilage grown in vitro by mesenchymal condensation. Proc Natl Acad Sci U S A 111, 6940, 2014.

11. Mollon, B., Kandel, R., Chahal, J., and Theodoropoulos, J. The clinical status of cartilage tissue regeneration in humans. Osteoarthritis Cartilage 21, 1824, 2013.

12. Hui, J.H., Goyal, D., Nakamura, N., Ochi, M., and Asian Cartilage Society. Cartilage repair: 2013 Asian update. Arthroscopy 29, 1992, 2013.

13. Johnstone, B., Alini, M., Cucchiarini, M., Dodge, G.R., Eglin, D., Guilak, F., et al. Tissue engineering for articular cartilage repair-the state of the art. Eur Cell Mater 25, 248, 2013.

14. Tuan, R.S., Chen, A.F., and Klatt, B.A. Cartilage regeneration. J Am Acad Orthop Surg 21, 303, 2013.

15. Gopal, K., Amirhamed, H.A., and Kamarul, T. Advances of human bone marrow-derived mesenchymal stem cells in the treatment of cartilage defects: a systematic review. Exp Biol Med (Maywood) 239, 663, 2014.

16. Darling, E.M., and Athanasiou, K.A. Rapid phenotypic changes in passaged articular chondrocyte subpopulations. J Orthop Res 23, 425, 2005.

17. Goldberg, A.J., Lee, D.A., Bader, D.L., and Bentley, G. Autologous chondrocyte implantation. Culture in a TGFbeta-containing medium enhances the re-expression of a chondrocytic phenotype in passaged human chondrocytes in pellet culture. J Bone Joint Surg Br 87, 128, 2005.

18. Lim, E.H., Sardinha, J.P., Myers, S., and Stevens, M. Latent transforming growth factor-betal functionalised electrospun scaffolds promote human cartilage differentiation: towards an engineered cartilage construct. $\underline{\text { Arch }}$ Plastic Surg 40, 676, 2013.

19. Munirah, S., Samsudin, O.C., Aminuddin, B.S., and Ruszymah, B.H. Expansion of human articular chondrocytes and formation of tissue-engineered cartilage: a step towards exploring a potential use of matrix-induced cell therapy. Tissue Cell 42, 282, 2010.

20. Hsieh-Bonassera, N.D., Wu, I., Lin, J.K., Schumacher, B.L., Chen, A.C., Masuda, K., et al. Expansion and redifferentiation of chondrocytes from osteoarthritic cartilage: cells for human cartilage tissue engineering. Tissue Eng Part A 15, 3513, 2009.

21. Liu, G., Kawaguchi, H., Ogasawara, T., Asawa, Y., Kishimoto, J., Takahashi, T., et al. Optimal combination of soluble 
factors for tissue engineering of permanent cartilage from cultured human chondrocytes. J Biol Chem 282, 20407, 2007.

22. Jakob, M., Demarteau, O., Schafer, D., Hintermann, B., Dick, W., Heberer, M., et al. Specific growth factors during the expansion and redifferentiation of adult human articular chondrocytes enhance chondrogenesis and cartilaginous tissue formation in vitro. J Cell Biochem 81, 368, 2001.

23. Ahmed, N., Iu, J., Brown, C.E., Taylor, D.W., and Kandel, R.A. Serum- and growth-factor-free three-dimensional culture system supports cartilage tissue formation by promoting collagen synthesis via Sox9-Col2a1 interaction. Tissue Eng Part A 20, 2224, 2014.

24. Taylor, D.W., Ahmed, N., Gan, L., Gross, A.E., and Kandel, R.A. Proteoglycan and collagen accumulation by passaged chondrocytes can be enhanced through side-byside culture with primary chondrocytes. Tissue Eng Part A 16, 643, 2010.

25. Ahmed, N., Taylor, D.W., Wunder, J., Nagy, A., Gross, A.E., and Kandel, R.A. Passaged human chondrocytes accumulate extracellular matrix when induced by bovine chondrocytes. J Tissue Eng Regen Med 4, 233, 2010.

26. Brittberg, M. Autologous chondrocyte transplantation. Clin Orthop Relat Res S147-S155, 1999.

27. Ahmed, N., Gan, L., Nagy, A., Zheng, J., Wang, C., and Kandel, R.A. Cartilage tissue formation using redifferentiated passaged chondrocytes in vitro. Tissue Eng Part A 15, 665, 2009.

28. Shinomura, T., Jensen, K.L., Yamagata, M., Kimata, K., and Solursh, M. The distribution of mesenchyme proteoglycan (PG-M) during wing bud outgrowth. Anat Embryol (Berl) 181, 227, 1990.

29. Kimata, K., Oike, Y., Tani, K., Shinomura, T., Yamagata, M., Uritani, M., et al. A large chondroitin sulfate proteoglycan (PG-M) synthesized before chondrogenesis in the limb bud of chick embryo. J Biol Chem 261, 13517, 1986.

30. Shepard, J.B., Gliga, D.A., Morrow, A.P., Hoffman, S., and Capehart, A.A. Versican knock-down compromises chondrogenesis in the embryonic chick limb. Anat Rec (Hoboken) 291, 19, 2008.

31. Hudson, D.M., Weis, M., and Eyre, D.R. Insights on the evolution of prolyl 3-hydroxylation sites from comparative analysis of chicken and Xenopus fibrillar collagens. PLoS One 6, e19336, 2010.

32. Matsumoto, K., Kamiya, N., Suwan, K., Atsumi, F., Shimizu, K., Shinomura, T., et al. Identification and characterization of versican/PG-M aggregates in cartilage. J Biol Chem 281, 18257, 2006.

33. Gregory, K.E., Keene, D.R., Tufa, S.F., Lunstrum, G.P., and Morris, N.P. Developmental distribution of collagen type XII in cartilage: association with articular cartilage and the growth plate. J Bone Miner Res 16, 2005, 2001.

34. Watt, S.L., Lunstrum, G.P., McDonough, A.M., Keene, D.R., Burgeson, R.E., and Morris, N.P. Characterization of collagen types XII and XIV from fetal bovine cartilage. J Biol Chem 267, 20093, 1992.

35. Hudson, K.S., Andrews, K., Early, J., Mjaatvedt, C.H., and Capehart, A.A. Versican G1 domain and V3 isoform overexpression results in increased chondrogenesis in the developing chick limb in ovo. Anat Rec (Hoboken) 293, 1669, 2010.

36. Choocheep, K., Hatano, S., Takagi, H., Watanabe, H., Kimata, K., and Kongtawelert, P. Versican facilitates chondrocyte differentiation and regulates joint morphogenesis. Biol Chem 285, 21114, 2010.
37. Gerecke, D.R., Foley, J.W., Castagnola, P., Gennari, M., Dublet, B., Cancedda, R., et al. Type XIV collagen is encoded by alternative transcripts with distinct $5^{\prime}$ regions and is a multidomain protein with homologies to von Willebrand's factor, fibronectin, and other matrix proteins. J Biol Chem 268, 12177, 1993.

38. Shaw, L.M., and Olsen, B.R. FACIT collagens: diverse molecular bridges in extracellular matrices. Trends Biochem Sci 16, 191, 1991.

39. Mazzorana, M., Cogne, S., Goldschmidt, D., AubertFoucher E. Collagenous sequence governs the trimeric assembly of collagen XII. J Biol Chem 276, 27989, 2001.

40. Wei, Y., Yang, E.V., Klatt, K.P., and Tassava, R.A. Monoclonal antibody MT2 identifies the urodele alpha 1 chain of type XII collagen, a developmentally regulated extracellular matrix protein in regenerating newt limbs. Dev Biol 168, 503, 1995.

41. Agarwal, P., Zwolanek, D., Keene, D.R., Schulz, J.N., Blumbach, K., Heinegard, D., et al. Collagen XII and XIV, new partners of cartilage oligomeric matrix protein in the skin extracellular matrix suprastructure. J Biol Chem 287, 22549, 2012.

42. Hemmavanh, C., Koch, M., Birk, D.E., and Espana, E.M. Abnormal corneal endothelial maturation in collagen XII and XIV null mice. Invest Ophthalmol Vis Sci 54, 3297, 2013.

43. Polacek, M., Bruun, J.A., Elvenes, J., Figenschau, Y., and Martinez, I. The secretory profiles of cultured human articular chondrocytes and mesenchymal stem cells: implications for autologous cell transplantation strategies. $\underline{\text { Cell }}$ Transplant 20, 1381, 2011.

44. Lee, W.D., Hurtig, M.B., Kandel, R.A., and Stanford, W.L. Membrane culture of bone marrow stromal cells yields better tissue than pellet culture for engineering cartilage-bone substitute biphasic constructs in a two-step process. Tissue Eng Part C Methods 17, 939, 2011.

45. Lee, J., Abdeen, A.A., Zhang, D., and Kilian, K.A. Directing stem cell fate on hydrogel substrates by controlling cell geometry, matrix mechanics and adhesion ligand composition. Biomaterials 34, 8140, 2013.

46. Zhu, J., Cai, Q., Zhang, X., Hu, X., Li, L., Wang, W., et al. Biological characteristics of mesenchymal stem cells grown on different topographical nanofibrous poly-Llactide meshes. J Biomed Nanotechnol 9, 1757, 2013.

47. Takahashi, T., Ogasawara, T., Asawa, Y., Mori, Y., Uchinuma, E., Takato, T., et al. Three-dimensional microenvironments retain chondrocyte phenotypes during proliferation culture. Tissue Eng 13, 1583, 2007.

Address correspondence to: Rita A. Kandel, MD

Department of Pathology and Laboratory Medicine Mount Sinai Hospital University of Toronto 600 University Avenue Toronto, ON M5G $1 X 5$

Canada

E-mail: rkandel@mtsinai.on.ca

Received: February 10, 2014

Accepted: September 15, 2014

Online Publication Date: December 12, 2014 


\title{
Osteoarthritis and Cartilage
}

Review

\section{The clinical status of cartilage tissue regeneration in humans}

\author{
B. Mollon $\dagger$, R. Kandel $\ddagger \S$, J. Chahal $\|$ ฯ, J. Theodoropoulos $\llbracket \#^{*}$ \\ $\dagger$ Department of Orthopaedic Surgery, University of Toronto, Toronto, Ontario, Canada \\ $\ddagger$ Department of Pathology and Laboratory Medicine, Mount Sinai Hospital, Toronto, Ontario, Canada \\ $\S$ Samuel Lunenfeld Research Institute, Mount Sinai Hospital, Toronto, Ontario, Canada \\ || University Health Network Arthritis Program, Toronto, Ontario, Canada \\ - University of Toronto Orthopaedic Sports Medicine Program, Women's Collage Hospital, Toronto, Ontario, Canada \\ \# Department of Orthopaedic Surgery, Mount Sinai Hospital, University of Toronto, Toronto, Ontario, Canada
}

\section{A R T I C L E I N F O}

\section{Article history:}

Received 30 April 2013

Accepted 28 August 2013

\section{Keywords:}

Cartilage

Tissue engineering

Autologous chondrocyte implantation

Chondrocytes

\begin{abstract}
S U M M A R Y
Purpose: To provide a comprehensive overview of the basic science and clinical evidence behind cartilage regeneration techniques as they relate to surgical management of chondral lesions in humans. Methods: A descriptive review of current literature.

Results: Articular cartilage defects are common in orthopedic practice, with current treatments yielding acceptable short-term but inconsistent long-term results. Tissue engineering techniques are being employed with aims of repopulating a cartilage defect with hyaline cartilage containing living chondrocytes with hopes of improving clinical outcomes. Cartilage tissue engineering broadly involves the use of three components: cell source, biomaterial/membranes, and/or growth stimulators, either alone or in any combination. Tissue engineering principles are currently being applied to clinical medicine in the form of autologous chondrocyte implantation (ACI) or similar techniques. Despite refinements in technique, current literature fails to support a clinical benefit of $\mathrm{ACI}$ over older techniques such as microfracture except perhaps for larger $(>4 \mathrm{~cm}$ ) lesions. Modern ACI techniques may be associated with lower operative revision rates. The notion that $\mathrm{ACI}$-like procedures produce hyaline-like cartilage in humans remains unsupported by high-quality clinical research.

Conclusions: Many of the advancements in tissue engineering have yet to be applied in a clinical setting. While basic science has refined orthopedic management of chondral lesions, available evidence does not conclude the superiority of modern tissue engineering methods over other techniques in improving clinical symptoms or restoring native joint mechanics. It is hoped further research will optimize ease of cell harvest and growth, enhanced cartilage production, and improve cost-effectiveness of medical intervention.
\end{abstract}

(c) 2013 Osteoarthritis Research Society International. Published by Elsevier Ltd. All rights reserved.

\section{Introduction}

Articular cartilage defects are commonly encountered in orthopedic practice but still represent a treatment challenge with inconsistent long-term results ${ }^{1}$. Articular cartilage is an avascular tissue composed of chondrocytes dispersed within an extracellular matrix (ECM) comprised of collagen and proteoglycans ${ }^{2}$. Found at the articulating end of bones, hyaline cartilage provides a low friction interface that also bears $\operatorname{load}^{3}$. Formed initially from

\footnotetext{
* Address correspondence and reprint requests to: J. Theodoropoulos, University of Toronto Orthopaedic Sports Medicine, 600 University Ave, Suite 476C, Toronto, Ontario M5G 1X5, Canada. Tel: 1-416-586-4800x8699; Fax: 1-416-586-8501.

E-mail addresses: brent.mollon@mail.utoronto.ca (B. Mollon), rkandel@ mtsinai.on.ca (R. Kandel), jchahal@utosm.com (J. Chahal), jtheodoropoulos@ mtsinai.on.ca (J. Theodoropoulos).
}

undifferentiated mesenchymal cells, chondrocytes synthesize cartilage matrix composed of $60 \%$ collagen (type II predominant), $25 \%$ proteoglycans, and $15 \%$ glycoproteins ${ }^{4}$. The composition of cartilage matures during progression to adulthood, resulting in a zonal organization of superficial, middle and deep calcified layers that are anchored into subchondral bone $e^{5}$. Overall, maturation results in a seven-fold increase in collagen cross-linking, and a $450 \%$ increase in the tensile and $180 \%$ increase in the compressive modulus of cartilage $e^{6,7}$. While chondrocytes are primarily involved in articular cartilage maintenance through the synthesis of ECM, overall cartilage homeostasis is thought to be the product of a complex interplay between joint mechanics, growth factors, hormones and aging 4 .

Although our understanding of these processes is evolving, a chondral lesion can simply be thought of as the inability of matrix synthesis to counter-act destructive forces placed on a joint. Once 
trauma or disease provokes an intra-articular destructive process, human adult articular cartilage has a limited ability to spontaneously heal, especially for larger defects ( $>3 \mathrm{~mm}$ ), defects that do not breach the subchondral plate, or in older patients ${ }^{4}$. Reasons for the ineffective reparative response after damage are thought to include the inability of chondrocytes to migrate to the site of injury, the avascular nature of cartilage, and the absence of a fibrin clot scaffold $^{1,8}$.

While a range of clinical options exist for the treatment of cartilage defects, the majority of current treatment options are aimed at symptom relief and fall short of the goal of recreating preinjury joint mechanics with the biologic capacity of long-term healing (see Table I for a summary of current management options). At one end of the spectrum, symptomatic relief may be obtained with oral analgesia, weight loss, physiotherapy to strengthen deconditioned muscles or arthroscopic chondroplasty, which aims to shave off the loose cartilage margins thought to be involved in mechanical joint irritation. These processes address pain, but fail to address the chondral lesion and thus are thought to not adequately address the longer-term sequela of cartilage injury: the development of osteoarthritis ${ }^{9}$. At the other end, joint arthroplasty can be performed in most major synovial joints to replace a severe osteoarthritic process with a metallic prosthesis. While this procedure affords good quality of life $(\mathrm{QOL})$, it is not appropriate for young individuals as the risk of failure increases over time and the functional limitations of a prosthesis are likely not adequate for an otherwise active or working individual. In between these two types of treatment modalities 'biological' cartilage treatments broadly attempt to fill the cartilage defect with stimulated fibrocartilage growth (i.e., microfracture) or a chondrocyte-containing plug [e.g., osteochondral transfer, mosaicplasty or autologous chondrocyte implantation $(\mathrm{ACI})]^{10}$.

The ideal treatment would reestablish the low friction properties of cartilage with the ability to resist wear over time by repopulating a lesion with chondrocytes able to produce a hyaline matrix that is fully integrated with surrounding host cartilage. The goal of creating integrative hyaline cartilage within a joint will theoretically improve joint mechanics and delay or even stop osteoarthritic progression within a joint. Hope lies in the area of tissue engineering to achieve this goal.

The purpose of this article is to describe the principles of tissue engineering in the context of cartilage regeneration in humans. Both the current status and future directions of tissue-engineered cartilage will be explored.

\section{Principles of cartilage tissue engineering}

Tissue engineering principles emerged in the late 1980s with the goal of reconstituting the structure and function of human tissues $^{8}$. This approach has since been investigated intensively and there is proof-of concept evidence to support cell-based regeneration of cartilage tissue ${ }^{11}$. With tissue engineering, researchers have been able to create biologically active, two or three-dimensional cartilage-like tissue complete with chondrocytes and supporting matrix that can fill a chondral lesion. Although complex, the overall process can be distilled down to three basic components: cells, scaffolds/matrix, and/or growth stimulators ${ }^{8}$. Cells must be capable of maintaining the articular chondrocyte phenotype or stimulate the differentiation of other cell types into chondrocytes and accumulate hyaline cartilage matrix. A structural matrix or scaffold will facilitate the formation of a cartilage matrix. Finally, growth or matrix stimulators in the form of biological, chemical or mechanical stimulation will encourage appropriate cellular growth and matrix synthesis on the scaffold in vivo or vitro ${ }^{8,11}$.

\section{Cell sources for chondral repair}

First and foremost, cartilage tissue engineering necessitates a large number of chondrocytes capable of creating hyaline cartilage ${ }^{11}$. Unfortunately, the cell source also serves as the main limiting factor to clinical translation as, due to low cellularity, only a small number of primarily obtained autologous chondrocytes can be directly harvested from an individual. As a result, several other sources of chondrocytes have been identified including passaged chondrocytes, induced pluripotent cells (IPCs), mesenchymal stromal cells (MSCs), and human embryonic stem cells (hESCs) ${ }^{11}$.

Table I

Current clinical options for the treatment of cartilage defects ${ }^{1,10}$

\begin{tabular}{|c|c|c|c|}
\hline Treatment & Description & Benefits & Limitations* \\
\hline Non-surgical & Oral analgesia, weight loss, physiotherapy & May avoid surgery & $\begin{array}{l}\text { Only masks symptoms, chronic use of pain } \\
\text { medications }\end{array}$ \\
\hline $\begin{array}{l}\text { Arthroscopic } \\
\text { chondroplasty }\end{array}$ & $\begin{array}{l}\text { Minimally invasive resection of loose cartilage } \\
\text { to decrease mechanical joint irritation }\end{array}$ & $\begin{array}{l}\text { Simple procedure, immediate } \\
\text { weight bearing }\end{array}$ & Only masks symptoms \\
\hline Microfracture & $\begin{array}{l}\text { Minimally invasive arthroscopic surgical } \\
\text { procedure that breaches the subchondral bone } \\
\text { with a pick to release osteoprogenitor cells } \\
\text { into a defect to encourage fibrocartilage growth }\end{array}$ & $\begin{array}{l}\text { Minimally invasive, no tissue } \\
\text { grafts required, only routine } \\
\text { surgical instruments needed, } \\
\text { used for lesions }<2.5 \mathrm{~cm}^{2}\end{array}$ & $\begin{array}{l}\text { Fibrocartilage biomechanically inferior to } \\
\text { hyaline cartilage, brief period of non-weight } \\
\text { bearing, unclear impact on development of } \\
\text { arthritis }\end{array}$ \\
\hline $\begin{array}{l}\text { Mosiacplasty/ } \\
\text { osteochondral } \\
\text { autograft transfer }\end{array}$ & $\begin{array}{l}\text { Uses multiple osteochondral autografts harvested } \\
\text { from the patients femur to fill an osteochondral } \\
\text { defect }\end{array}$ & $\begin{array}{l}\text { No allograft, theoretically fills } \\
\text { in with hyaline cartilage. } \\
\text { Used to treat lesions from } \\
1-4 \mathrm{~cm}^{2}\end{array}$ & $\begin{array}{l}\text { Graft-site mismatch may not recreate native } \\
\text { joint mechanics, graft-site morbidity, cannot } \\
\text { treat large lesions, lack of integration with } \\
\text { surrounding tissues }\end{array}$ \\
\hline $\mathrm{ACI}$ & $\begin{array}{l}\text { Harvested chondrocytes are cultured prior to } \\
\text { being re-implanted into the defect. An evolving } \\
\text { procedure due to advances in tissue engineering }\end{array}$ & $\begin{array}{l}\text { May produce hyaline cartilage, } \\
\text { can treat lesions } 2-10 \mathrm{~cm}^{2}\end{array}$ & $\begin{array}{l}\text { Graft delamination, periosteal hypertrophy, } \\
\text { questionable ability to produce hyaline } \\
\text { cartilage }\end{array}$ \\
\hline $\begin{array}{l}\text { Osteochondral } \\
\text { allograft transfer }\end{array}$ & $\begin{array}{l}\text { Uses allogenic (cadaveric) osteochondral tissue } \\
\text { to fill defect }\end{array}$ & $\begin{array}{l}\text { Can treat large lesions, no graft- } \\
\text { site morbidity }\end{array}$ & $\begin{array}{l}\text { Allogenic tissue (potential for disease } \\
\text { transmission), size/depth mismatch, } \\
\text { questionable chondrocyte viability, lack of } \\
\text { integration with surrounding tissues }\end{array}$ \\
\hline Joint arthroplasty & $\begin{array}{l}\text { Resects and replaces arthritic bone with an } \\
\text { artificial joint, most commonly metal implants } \\
\text { (i.e., cobalt chrome) separated by a polyethylene } \\
\text { liner }\end{array}$ & $\begin{array}{l}\text { Pain relief, variable return } \\
\text { to function }\end{array}$ & $\begin{array}{l}\text { Variable return to function/activity } \\
\text { limitations, infection, Implants wear out } \\
\text { over time (need for re-operation), cannot } \\
\text { completely recreate native anatomy or } \\
\text { mechanics }\end{array}$ \\
\hline
\end{tabular}

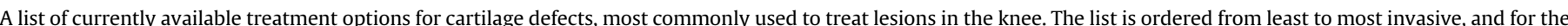
treatment of smallest to largest defects. The bulk of this article will focus on ACI and its evolution due to research into cartilage tissue engineering.

* Note: no therapy has been shown to alter the natural history of a chondral lesion (i.e., progression to osteoarthritis), thus serving as a limitation for all therapies. 
Stimulatory procedures, $A C I$, minced and passaged chondrocytes

Marrow stimulation techniques, which can be considered a precursor to tissue engineering, include osteochondral drilling, abrasion chondroplasty and microfracture ${ }^{1}$. These techniques all seek to stimulate the release of chondroprogenitor cells into the defect to encourage the formation of fibrocartilage (composed of type I and type II collagen). While often the simplest option for small isolated defects, fibrocartilage is mechanically inferior to hyaline cartilage (composed of type II collagen $)^{12}$. For that reason, marrow stimulation techniques can be considered a pain-relieving procedure that at most slows the progression towards osteoarthritis ${ }^{9,13}$.

Osteoarticular transplant procedures use native chondrocytecontaining cartilage with underlying bone. Given the described complexity of the structure of cartilage, the allure of repairing a chondral lesion with structurally mature tissue obtained from either a cadaver (allogeneic transplant) or non-weight bearing zone of the articular surface from the patient's own body (autologous transplant) is understandable. Although very useful, concerns over donor site morbidity, chondrocyte viability, disease transmission from allogeneic tissue, and lack of integration with the margins of a chondral defect are challenges that remain to be overcome by such procedures $^{14-16}$.

The procedure most related to human tissue engineering is ACI. First described in rabbits by Grande et al. ${ }^{17}$ and later in humans by Brittberg et $a l^{18}$ to treat knee chondral lesions, ACI uses arthroscopically harvested chondrocytes that are subsequently cultured in monolayer (so-called 'passaged chondrocytes'). The chondrocyte suspension is then implanted into the defect and sutured under a watertight periosteal patch. This treatment, which requires two operations spaced six to 8 weeks apart, was originally indicated in patients with focal lesions $2-10 \mathrm{~cm}^{2}$ in size $\mathrm{e}^{18}$. Randomized clinical trials have yielded mixed results on the ability of ACI-like procedures to produce enhanced structural repair over microfracture, with minimal clinical differences at 5 years ${ }^{19,20}$. While clinical results are generally favorable, risks include periosteal hypertrophy, delamination of the graft and arthrofibrosis ${ }^{21,22}$. In addition, the ability of ACI to reliably produce hyaline-like cartilage has been challenged, with some animal models suggesting that some healing is stimulated by the ingrowth of progenitor cells from breached subchondral bone or from the periosteal patch ${ }^{23,24}$. Furthermore, culturing chondrocytes in monolayer culture to increase cell numbers, known as passaged chondrocytes, results in a decreased capacity to produce hyaline-like matrix due to chondrocyte dedifferentiation ${ }^{25,26}$.

A variant of $\mathrm{ACI}$ is found in procedures utilizing particulated articular cartilage. Animal and subsequent clinical studies have demonstrated minced cartilage without bone or cell culture can provide a cell source for cartilage repair ${ }^{27,28}$. Chondrocytes from minced cartilage display a standard chondrocyte phenotype and are through to migrate from the graft ECM, multiply and form hyalinelike cartilage integrated with native tissue ${ }^{28}$. Available commercial products include deNovo NT (Zimmer, Inc., Warsaw, IN) and Cartilage Autograft Implantation System (CAIS; DePuy Mitek Inc., Raynham, MA $)^{29}$. CAIS utilizes autogenous cartilage tissue harvested intra-operatively and distributed on a polycaprolactone/polyglycolic acid scaffold secured under a polydiaxone mesh, while deNovo NT utilizes particulated viable juvenile allograft hyaline cartilage pieces that are secured into a defect with fibrin glue. Both products and have found promising short-term results ${ }^{29}$.

The application of tissue engineering principles has resulted in the progressive refinement of the ACI-like procedures to address some of the above shortcomings. For example, we have shown that passaged chondrocytes that have adapted a fibroblast-like morphology can undergo redifferentiation when co-cultured with non-passaged (or primary) chondrocytes and reacquire the ability to form hyaline cartilage $\mathrm{e}^{25,26}$. The mechanism underlying this redifferentiation is unclear, but may be related to direct cell-cell communication, ECM microenvironment produced by chondrocytes, or paracrine signaling ${ }^{30,31}$. Regardless, these cells could then be used to redifferentiate other passaged chondrocytes, thus forming a stable phenotype that could be utilized in ACI procedures $^{26}$. Additional studies are required to evaluate the efficacy of our co-culture method in vivo.

The evolution of ACI has resulted in four described 'generations' that have been expanded upon in other reviews ${ }^{32,33}$. We propose the following divisions between ACI generations in Table II, with each generation using more advanced tissue engineering technologies. Clinically, each generation is thought to represent a move towards less patient morbidity (i.e., arthroscopic instead of open procedures; or one-stage operations) or the enhanced production of hyaline cartilage.

\section{MSCS}

MSCs are multipotent cells capable of differentiation into osteocyte, adipocyte and chondrocyte lineages under the appropriate conditions ${ }^{34}$. Defined by their expression of certain cell surface molecules (i.e., CD73, CD105, CD90) and their ability to grow as adherent fibroblast-like cells in vitro, MSCs are referred to as stromal cells instead of previously named stem cells as they are ultimately restricted in the type of cells into which they can differentiate ${ }^{35}$.

The process of collecting, isolating and growing MSCs from various sources is beyond the scope of this article (see review by Archer et $a l^{36}$ ). In brief, cells are obtained via bone marrow aspiration or tissue enzymatic degradation and expanded in culture.

Table II

Generational development of autologous chondrocyte-like implantation techniques

\begin{tabular}{|c|c|c|}
\hline Generation & Description & Defining features \\
\hline First & $\begin{array}{l}\text { Autograft chondrocytes are obtained via arthroscopy, expanded in culture, and re-implanted } \\
\text { under a periosteal or collagen patch during a second operation. }\end{array}$ & Periosteal/collagen patch used $A N D$ no scaffolds \\
\hline Second & $\begin{array}{l}\text { Autograft chondrocytes are obtained via arthroscopy, chondrocytes are expanded on a scaffold, } \\
\text { and the chondrocyte/scaffold complex is inserted into the knee at a later operation without } \\
\text { a periosteal/collagen patch. }\end{array}$ & Basic scaffolds $A N D$ no periosteal patch \\
\hline Third & $\begin{array}{l}\text { Introduces either chondro-conductive or -inductive scaffolds, xeno/allogeneic cells, biphasic } \\
\text { graft constructs, or mechanically conditioned chondrocytes during the culturing process. }\end{array}$ & $\begin{array}{l}\text { Utilizes all three components of tissue engineering } \\
\text { (introduces growth factors/mechanical conditioning) } \\
O R \text { introduces non-self cell types } O R \text { attempts to } \\
\text { reproduce zonal architecture of mature cartilage }\end{array}$ \\
\hline Fourth & Utilizes stromal cells, stem cells, or gene therapy to produce chondrocytes. & Stem cells/gene therapy for chondrogenesis \\
\hline
\end{tabular}

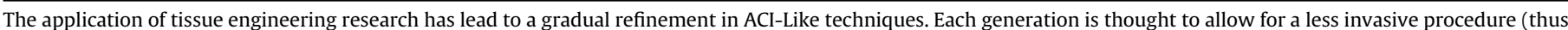
decreasing patient morbidity), increase the reliability of hyaline cartilage formation, improve graft uptake or decrease the number of surgical procedures required.

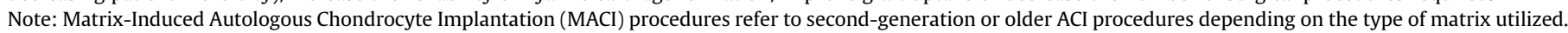


Flow cytometry may be used to select cells expressing known MSC surface markers. Culture conditions are then optimized to induce differentiation into the desired cell line, ${ }^{36}$ in this case chondrocytes.

Bone marrow represents the main source of MSCs (so-called bmMSCs), although umbilical cord, adipose tissue, synovial membrane and articular cartilage represent alternate sources. It should be noted that MSCs obtained from varying cellular sources express differing densities and types of cell surface proteins/markers ${ }^{36}$. For example, CD34+ is identified only on adipose derived MSCs, Tissue Non-Specific Alkaline Phosphatase (TNAP) is exclusively found on bmMSCs, and Stage Specific Embryonic Antigen 4 (SSEA-4) is expressed by placenta derived MSCs ${ }^{36,37}$. These differences may reflect differences in chondrogenesis noted amongst MSC cell lines in some studies. For example, a comparison of bone marrow, adipose derived, muscle derived or synovial derived stromal cells obtained from the same individual revealed synovial derived cells had a superior potential for chondrogenesis ${ }^{38}$ and produced larger cartilage aggregates over time ${ }^{39}$ when compared with bmMSCs. The clinical utility of this finding is unclear, as synovium-derived MSCs have yet to be used in humans ${ }^{40}$, and as previously mentioned the cellular composition and presence of environmental stimuli may be as important as the origin of the stromal cell. Cellular responses to growth factors or scaffolds may differ not only between different sources of MSCs but also within them. For example, Battula et al. utilized monoclonal antibodies to identify antigens associated with rapidly growing bmMSCs: CD271 and $\mathrm{CD}^{4} 6^{41}$. Cells expressing both antigens proliferated more than 30 times faster than an unsorted pool of bmMSCs. The results of this study also suggest that cells expressing CD271, CD56 and TNAP preferably generated chondrocytes and displaying decreased adipogenic potential ${ }^{41}$.

MSCs are commonly utilized in tissue engineering, with bmMSCs being the most common cell source utilized clinically in humans ${ }^{36}$. For example, in an observational cohort study by Nejadnik et al. ${ }^{42} \mathrm{ACI}$ was compared with a group that received a similar treatment using autologous bmMSCs instead of chondrocytes. The authors concluded there was no difference in clinical outcome between groups at 24 months after surgery ${ }^{42}$. Additionally, Wakitani et al. $^{43}$ utilized culture expanded autologous bmMSCs embedded on a collagen sheet for the treatment of patellofemoral joint chondral defects in a small case series. The bmMSCs were transplanted into the defect and secured with a periosteal graft or synovium (similar to first generation ACI techniques), with symptomatic improvement noted for as long as 27 months ${ }^{43}$. Longer-term follow-up studies have confirmed this to be a safe procedure without development of tumor or infections in a group of 40 patients over 11 years ${ }^{40}$. These results suggest at the very least equivalence in clinical outcome between implantation of chondrocytes or bmMSCs in ACI-type procedures in terms of shortterm symptomatic relief. While biopsies obtained during second look arthroscopies suggest the presence of hyaline-like cartilage in both the bmMSCs and ACI group in one trial ${ }^{42}$, this is based on a small subset of the original study population requiring arthroscopy for symptomatic knees. Thus, true superiority of bmMSCs over earlier generation $\mathrm{ACI}$ techniques remains unproven.

\section{ESCS}

ESCs are defined by their ability to proliferate in an undifferentiated state for a prolonged period while maintaining the capability to differentiate into any mature cell in the body, including chondrocytes. First described by Thomson et al. ${ }^{44}$, ESCs are first obtained from the inner cell mass of blastocyst-stage embryos. After this, progression towards viable chondrocytes can occur either through the formation of an embryoid body (EB) and subsequent selection of mesodermal cells, or by first transforming ESCs into MSCs before pursuing a chondrogenic phenotype via growth factors or sorting by surface antigens (see previous section $)^{45,46}$. Challenges with both these techniques include difficultly in guaranteeing a pure population of chondrocytes when manipulating pluripotent cells, the potential for ESCs that have differentiated to chondrocytes to undergo de-differentiation into other lineages (i.e., skeletal muscle), and to date no one has produced sufficient hyaline cartilage tissue from ESCs suitable for joint resurfacing ${ }^{47-49}$. Safety concerns are paramount, as undifferentiated residual ESCs are known to be tumorogenic ${ }^{45}$. Recent animal studies have suggested that injection of ESCs into a joint cavity results in teratoma formation, while localized injection into osteochondral defects does not ${ }^{50,51}$. Additionally, joint immobilization may encourage tumor formation while joint mobility encourages chondrogenesis ${ }^{52}$. While showing promise, additional work is required to better understand the factors involved in producing a clinically suitable, homogenous chondrocyte population from hESCs. Indeed, no trial in humans has as yet been published, although animal studies have been reported ${ }^{53,54}$.

Induced Pluripotent Stem Cells (iPSCs) are an alternate method of creating cells with ESC-like properties. As originally described by Takahashi and Yamanaka ${ }^{55}$, mouse fibroblasts can be transduced with the transcription factors Oct3/4, Sox2, Klf4 and c-Myc, transforming them into cells with ESC-like pluripotency. IPSC express ESC cell marker genes and demonstrate ESC-like growth capabilities, including potential for teratoma formation. Since this discovery, many other cell types have been induced to acquire ESC-like phenotype $^{56}$. Recently Wei et al. ${ }^{57}$ described the transdifferentiation of human chondrocytes into iPSCs. The development of DNA alterations and genomic instability in iPSCs are issues that need to be addressed as well before these technological advances can be applied clinically ${ }^{56}$.

\section{Scaffolds}

Scaffolds are three-dimensional chondro-condusive biomaterials which facilitate chondrocyte number expansion and/or organization while also providing a mechanically stable support for human chondrocyte implantation ${ }^{58}$. Of note, 'chondro-conductive' substances support chondrocyte growth whereas 'chondro-inductive' substances induce the differentiation to, and maintenance of, the chondrogenic cellular phenotype. Safran et al. ${ }^{58}$ listed the requirements for the ideal scaffold including: biocompatible, biodegradable, permeable, noncytotoxic, mechanically stable, able to support chondrocyte growth, versatile, readily available and easy to manufacture. Additionally, appropriate porosity is considered another characteristic, with pore sizes between $100 \mu \mathrm{m}$ and $300 \mu \mathrm{m}$ thought to best optimize cellular seeding and differentiation while facilitating waste/nutrient dispersion ${ }^{59}$. Scaffolds currently represent a key component in chondrogenic differentiation of the aforementioned cell lineages as the three-dimensional environment is believed to facilitate the cellular and cell-matrix interactions encouraging chondrogenesis ${ }^{60}$. For that reason, the scaffolds have been used to augment microfracture or ACI-type procedures by facilitating chondrocyte transfer and speeding graft incorporation with the ultimate hope of increasing the proportion of 'hyaline' or 'hyaline-like' cartilage ${ }^{10,61,62}$.

Available scaffolds fall into one of four broad categories: protein, carbohydrate, synthetic and composite. Protein-based scaffolds include collagen, gelatin and fibrin; carbohydrate polymers include hyaluronan, alginate, alginate, and polylactic/polyglycolic acids; and synthetic scaffolds include Teflon, carbon fiber, Dacron, and hydroxyapatite ${ }^{58}$. 
Clinical use of scaffolds in cartilage regeneration

One of the most commonly used scaffolds is collagen, a natural scaffold which also contains sites for cellular adhesion and has been shown to influence chondrocyte differentiation ${ }^{63,64}$. Collagen scaffolds have also been utilized to enhance marrow stimulation techniques, with scaffolds being inserted post-microfracture as a one-step procedure. One commercial example is Chondro-Gide ${ }^{\circledR}$ (Geistlich Pharma AG, Wolhusen, Switzerland), a I/III collagen scaffold used in a technique termed Autologous Matrix-Induced Chondrogenesis $\left(\mathrm{AMIC}^{\circledR}\right.$ ) with promising non-comparative 2-year results but a paucity of data suggesting structural superiority over microfracture alone ${ }^{62}$. Collagen scaffolds have also been used instead of periosteal patches to secure cultured chondrocytes to cartilage defects in ACI procedures (see NeoCart ${ }^{\circledR}$, Histogenics, Waltham, MA $)^{63}$ and have displayed promising short-term results $^{63,64}$, but longer-term outcomes are required.

Hyaluronic acid-based scaffolds such as Hyalograft ${ }^{\circledR} \mathrm{C}$ (Anika Therapeutics, Bedford, MA) have also been utilized in cartilage regeneration $^{61}$. These carbohydrate-based polymers have the proposed benefit of being fully resorbed in 3 months after being degraded to hyaluronan and are thought to encourage chondrogenic differentiation as evident by an increased presence of type II collagen and aggrecan with a decrease in type I collagen ${ }^{65}$. While published clinical trials suggest good outcomes with hyaluronic acid-based grafts at 3 years after implantation, with a high percentage displaying hyaline or "hyaline-like" cartilage 18 months post implantation $^{61}$, this product is not available in North America and was recently withdrawn from the European market by the manufacturer.

Exciting potential exists for hydrogel scaffolds. Hydrogels are a liquid polymer that can be stimulated to undergo cross-linking to form a water-insoluble gel ${ }^{66}$. Materials investigated for this property include: alginate, fibrin, and synthetic polymers of polyethylene oxide and poly propylene oxide. There are many theoretical benefits to this technology: the ability of growth factors to diffuse through the gel, water content that mimics native cartilage, and the injectable nature that facilitates arthroscopic insertion. One hydrogel in clinical use is Gelrin $C^{\circledR}$ (Regentis, Haifa, Isreal). Gelrin $C^{\circledR}$ is a bioabsorbable photopolymerized hydrogel of polyethylene glycol diacrylate bound to fibrinogen and degrades within 6-12 months ${ }^{67}$. It is injected into a previously microfractured defect as a gel that polymerizes in situ. In vitro, Gelrin $C^{\circledR}$ exhibits innate chondrogenic and osteoconductive potential, is nonimmunogenic, and in an ovine model demonstrated type II collagen and proteoglycan synthesis in treated vs untreated defects $^{68}$. This product is being investigated in an ongoing multicenter clinical trial.

While chitosan/glycerol copolymer marketed as BST-Cargel ${ }^{\circledR}$ (Piramal Healthcare Ltd, Vikhroli West, Mumbai), has shown promise in previous studies ${ }^{69}$ and is being investigated in phase III clinical trials, few other types of scaffolds have been clinically investigated in humans.

\section{Growth factors}

As chondrocyte or stromal cell growth is regulated by an interplay between mechanical and chemical stimuli ${ }^{70}$, guided utilization of growth factors is advantageous when engineering cartilage. Growth factors are considered to be chondro-inductive substances and the factors commonly associated with cartilage growth and maturation fall into one of three broad categories: the Transforming Growth Factor- $\beta$ (TGF- $\beta$ ) family, Insulin-like Growth Factors (IGFs) and Fibroblast Growth Factor (FGF) ${ }^{70,71}$.
Table III

Known actions of growth factors in chondrogenesis

Transforming Growth Factor B (TGF- $\beta$ ) Superfamily ${ }^{7}$

Anabolic effect on chondrocytes

Maintains chondrocyte phenotype

Redifferentiates passaged chondrocytes

TGF- $\beta 1$ promotes cell proliferation and inhibits matrix metalloproteinases ${ }^{84}$

GDF-5 upregulates GAG and type II collagen production by hMSC derived chondrocytes ${ }^{85}$.

Bone Morphogenic Proteins (BMPs; Member of TGF- $\beta$ Superfamily)

Encourage undifferentiated mesenchymal cells towards chondrocyte phenotype $^{86}$

BMP-2 enhances redifferentiation of passaged chondrocytes ${ }^{87}$

BMP-4 and BMP-6 increase type II collagen and the accumulation of proteoglycans while decreasing type I collagen synthesis ${ }^{88,89}$

BMPs-2, -12 (GDF-7) and -13 enhanced collagen, GAG and cellular growth of cultured chondrocytes ${ }^{90}$.

IGFs

IGF-1 encourages chondrocyte proliferation ${ }^{91}$

IGF-1 (with TGF- $\beta 1$ ) promotes proteoglycan accumulation, type II collagen synthesis and maintenance of chondrocyte phenotype ${ }^{92}$

IGF-1 binds to proteoglycans via IGF-binding protein, thus being release to counter-act cartilage breakdown during periods of catabolism ${ }^{93}$

Insulin promotes chondrogenesis ${ }^{94}$ FGF

$(+/-)$ Mixed results on promotion of cartilage repair ${ }^{95,96}$

Encourages articular cartilage cell number expansion (in monolayer) ${ }^{97}$

(-ve) Suppresses proteoglycan synthesis ${ }^{98}$

(-ve) Flattens chondrocytes, encourages fibroblast morphology ${ }^{99}$

(-ve) Inhibits terminal differentiation of articular chondrocytes ${ }^{100}$

This table explores our evolving knowledge of major growth factors in chondrocyte regeneration.

The known actions of major chondrogenic growth factors are summarized in Table III. Broadly, these factors are thought to be involved in maintaining chondrocyte phenotype during monolayer passaging, encouraging chondrogenic differentiation of stem cells or stromal cells, maintaining chondrocyte phenotype, and encouraging collagen type II synthesis ${ }^{70-72}$. For example, human ESCs and mesodermal cells undergo chondrogenic differentiation in the presence of growth factors such as TGF- $\beta$ and BMP- $2^{48,49,73}$. Additionally, cell lines can be genetically engineered to express proteins whose goal is to enhance chondrogenic matrix production, such as type II collagen. For example, Kuroda et al. ${ }^{74}$ genetically engineered MDSCs to express BMP-4 and found type II collagen was expressed as early as 4 weeks in rats. Cell lineage also impacts growth factor activity. For example, aggrecan upregulation occurs when bmMSCs are exposed to TGF- $\beta 3$, while BMP-6 is needed to upregulate aggrecan on adipose derived $\mathrm{MSCs}^{75}$. Our clinical understanding of the roles of growth factors in the production of an articular chondrocyte phenotype in humans is evolving, but additional work is required.

Another way to administer growth factors in a less directed manner is Platelet-Rich Plasma (PRP), a growth factor-rich concentrate that can be easily acquired through centrifugation of patient's own blood creating an autologous preparation of serum with high concentrations of platelets, cytokines, and growth factors. The application of PRP acts to amplify the concentration of chemical mediators in the microenvironment of the injured area such as TGF- $\beta 1$, IGF-1, Vascular Endothelial Growth Factor (VEGF), and Platelet-Derived Growth Factor (PDGF), among others ${ }^{71}$. In vitro studies maintaining chondrocytes in the presence of PRP instead of fetal bovine serum have shown increased proliferation of human chondrocytes $^{76}$. Additionally, MSC's cultured in the presence of PRP can demonstrate differentiation towards chondrogenic and osteogenic lineages ${ }^{77,78}$. This technique may be used in the future as a way to deliver autologous growth factors to chondrocytes expanded in vitro prior to in vivo use. 


\section{Clinical impact}

It is hoped that advances in the three components of tissue engineering will lead to improved patient outcomes. Unfortunately, most developments in cartilage tissue engineering have yet to translate into measurable clinical gains or have yet to be applied to human populations due to novelty and/or safety concerns. The main orthopedic utilization of tissue engineering has focused on enhancing either marrow stimulation (i.e., AMIC) or ACI-type techniques.

Many recent systematic reviews on ACI have been published, most often comparing $\mathrm{ACI}$ to stimulatory (i.e., microfracture) techniques ${ }^{22,79-82}$. Vasiliadis et al. ${ }^{80}$ conducted a systematic review of randomized trials comparing various ACI treatments to other available treatment options (e.g., microfracture, mosaicplasty). They identified nine trials and found no superiority of $\mathrm{ACI}$ over other treatments. Nonetheless, they concluded that the evidence was of poor quality and too heterogeneous to make any definitive clinical recommendations ${ }^{80}$. A similar review of nine studies was conducted by Vavken et al. ${ }^{81}$. In contrast to the previous review, their data suggested that among high-quality trials, ACI resulted in better clinical outcomes and tissue quality when compared to osteochondral grafts. However, the authors noted that the differences between groups were small and may not reflect clinical significance, and ultimately concluded that additional research is required $^{81}$.

Harris et al. ${ }^{82}$ elaborated on differences between studies in their review of level I and II evidence. Of seven studies comparing microfracture to $\mathrm{ACI}$, they found three trials showed better clinical results with $\mathrm{ACI}$ after $1-3$ years follow-up, one study reporting better results after microfracture at 2 years, and three trials reporting no difference after $1-5$ years. They found a defect size of $>4 \mathrm{~cm}^{2}$ predicted better outcomes with $\mathrm{ACI}$ when compared to other treatments. There was no apparent difference between open or arthroscopic procedures, or first and second-generation $\mathrm{ACI}$ techniques ${ }^{82}$.

Clinicians must also consider the complications of a procedure before making a recommendation. Harris et al. ${ }^{22}$ reviewed all failures and complications from $\mathrm{ACl}$ therapies published in 82 studies. An overall failure rate of $5.8 \%$ was noted for $\mathrm{ACI}$ procedures, with a mean time to failure at 22 months. Techniques utilizing periosteal patches had the highest failure rates (7.7\%), with lower failure rates for all arthroscopic procedures (3.3\%) or those using secondgeneration $\mathrm{ACI}$ techniques (0.83\%). ACI techniques using periosteal patches were associated with an unplanned re-operation rate of $27 \%$, which decreased to $5 \%$ in second-generation $\mathrm{ACI}$, and $1.4 \%$ in all arthroscopic second-generation ACI techniques ${ }^{22}$. Taken along with the preceding reviews, the available literature has yet to identify a functional benefit to evolving $\mathrm{ACI}$ techniques but the overall complication rates and need for re-operation has decreased in all arthroscopic and second-generation techniques ${ }^{22,80-82}$.

Available data on third generation techniques are mostly limited to prospective safety trials ${ }^{63,83}$. Crawford et al. $^{63}$ evaluated the safety of the third generation NeoCart ${ }^{\circledR}$ procedure in a small prospective trial. The eight enrolled patients had improved pain, function and range of motion at 2 years. MRI-measured defect fill was found to be $67-100 \%$ in six patients; $33-66 \%$ in one patient and less than $33 \%$ in one patient. No serious complications were associated with the implant. A small sample and lack of a comparison group ultimately limited the strength of this study ${ }^{63}$. Cole et al. $^{83}$ presented a randomized controlled trial (RCT) involving 29 patients to establish the safety of using the Cartilage Autograft Implantation System (CAIS; DePuy Mitek, Inc., Raynham, MA) when compared to microfracture. CAIS utilizes minced autologous hyaline cartilage placed on an absorbable polyglycolic acid- polycaprolactone scaffold and affixed using absorbable polydiaxonone staples. The authors found general improvement in clinical outcomes in both groups, although the CAIS group significant improvements in the clinical rating scales over the microfracture group at 24 months of follow-up. Radiographic evaluation of lesion fill and tissue integration was similar between groups. The microfracture group had significantly higher rates of intralesional osteophyte formation at 6 and 12 months. From this they concluded the CAIS is safe and effective but acknowledged their study was limited by small sample size, and may have been influenced by differences between study populations (more patients with acute onset of symptoms, more men and more full-time workers in the CAIS group $)^{83}$. Neither of the above trials discussed the histological quality of the repair tissue (i.e., fibrocartilage vs hyaline-like cartilage $)^{63,83}$.

Considering the high cost associated with engineering chondrocytes with equivocal clinical data, there has only been one study focusing on the cost-effectiveness of these therapies. While Clar et al. $^{79}$ attempted a cost-comparison analysis in their systematic review of four RCTs, they were unable to generate conclusions due to limited evidence. They acknowledge that the QOL gain of ACI would need to be $70-100 \%$ greater than microfracture over 2 years, or alternatively $10-20 \%$ maintained over 10 years, to justify the use of $\mathrm{ACI}^{79}$. While it is hoped that the theoretical benefit of $\mathrm{ACI}$ in the generation of durable hyaline cartilage may justify its use, clinical evidence demonstrating benefit of $\mathrm{ACI}$ over other techniques in this area is sparse. Furthermore, long-term studies are required to support the assertion that the hyaline cartilage (vs fibrocartilage) results in improved long-term biomechanical properties that delays or prevents the development of osteoarthritis. Thus, based on the above economic analysis and available long-term data, the costeffectiveness analysis does not favor ACI over other less costly procedures that are potentially as efficacious.

\section{Conclusions and future research}

Current clinical research does not support a functional benefit of ACI techniques over older techniques like microfracture ${ }^{2,80,81}$. There is, however, a trend towards less complications or need for reoperation in all arthroscopic and newer-generation ACI techniques $^{22}$. Histological support for 'biomechanically superior' hyaline cartilage filling the defect in ACI procedures is lacking, as is evidence that the presence of this tissue ultimately delays or halts the development of osteoarthritis ${ }^{79,81}$. At this time many of the potential cell sources described above are still experimental, and may be decades away from clinical practice, if at all.

It is assumed that a multifactorial tissue engineering approach to cartilage regeneration is ideal - combining cells, and scaffold to create a biologically active graft. The role of growth factors is still controversial. There are many questions that still need to be answered if tissue engineering is to be utilized to repair a chondral defect with articular cartilage containing biologically active cells. For example, which cell when differentiated to a chondrocyte produces a matrix most similar to native hyaline cartilage and will this tissue decrease the risk of arthritis in those with osteochondral lesions? How will our evolving understanding of growth factors and scaffolds impact osteochondral repair? What conditions are necessary to encourage integration between native cartilage and the implanted graft? Are biphasic implants the best way to encourage stable integration of grafts? Do we need to recapitulate cartilage zonal organization with a deep calcified zone to facilitate integration and weight bearing?

The literature suggests that single staged procedures, all arthroscopic techniques, and avoiding periosteal patches appear to impact complication and revision rates, but there are many other 
questions that arise when the above is to be applied clinically. Is there any functional benefit of newer-generation $\mathrm{ACI}$ techniques? How does scaffold-augmented microfracture compare to ACI procedures? What is the optimal post-operative rehabilitation process? Is there a long-term impact on rates of osteoarthritis? Is there a way to make the process more financially feasible?

As stated in this review: our understanding of the factors influencing optimization and application of cartilage tissue engineering is expanding. However, the clinical impact of this research has yet to be truly appreciated as these findings have yet to be translated into human use. As new techniques or products are introduced, decisions on their utility must be predicated on evidence based medicine and functional outcomes. Each new advance should also be scrutinized for ease of cell harvest and growth, quality of cartilage produced, and overall cost-effectiveness. Through all of these ongoing efforts and developments, cartilage tissue engineering should be a powerful tool for the treatment of chondral defects.

\section{Contributions}

All authors (BM, RK, JC \& JT) were involved in the conception of this manuscript. One author (BM; email: brent.mollon@mail. utoronto.ca) drafted the article and takes responsibility for the integrity of the work as a whole. All other authors (RK, JC \& JT) critically appraised the article for important intellectual content and revised it accordingly. All authors approved the final version of the manuscript.

\section{Role of the funding source}

No funds were received for the preparation of this manuscript.

\section{Conflict of interest}

Authors BM, RK and JC have no financial disclosures or notable competing interests. Author JT serves as a consultant for Zimmer and for Smith and Nephew, has received speaker honorariums from ConMed Linvatec, and receives fellowship funding from Smith and Nephew.

\section{Acknowledgments}

None.

\section{References}

1. Mithoefer K. Complex articular cartilage restoration. Sports Med Arthrosc Rev 2013;21(1):31-7.

2. Heinegard D. Proteoglycans and more-from molecules to biology. Int J Exp Pathol 2009;90(6):575-86.

3. Adams MA, Dolan P, McNally DS. The internal mechanical functioning of intervertebral discs and articular cartilage, and its relevance to matrix biology. Matrix Biol J Int Soc Matrix Biol 2009;28(7):384-9.

4. Buckwalter JA, Mankin HJ, Grodzinsky AJ. Articular cartilage and osteoarthritis. Instr Course Lect 2005;54:465-80.

5. Allan KS, Pilliar RM, Wang J, Grynpas MD, Kandel RA. Formation of biphasic constructs containing cartilage with a calcified zone interface. Tissue Eng 2007;13(1):167-77.

6. Williamson AK, Chen AC, Masuda K, Thonar EJ, Sah RL. Tensile mechanical properties of bovine articular cartilage: variations with growth and relationships to collagen network components. J Orthop Res 2003;21(5):872-80.

7. Williamson AK, Chen AC, Sah RL. Compressive properties and function-composition relationships of developing bovine articular cartilage. J Orthop Res 2001;19(6):1113-21.
8. Hunziker EB. Articular cartilage repair: basic science and clinical progress. A review of the current status and prospects. Osteoarthritis Cartilage 2002;10(6):432-63.

9. Widuchowski W, Lukasik P, Kwiatkowski G, Faltus R, Szyluk K, Widuchowski J, et al. Isolated full thickness chondral injuries. Prevalance and outcome of treatment. A retrospective study of 5233 knee arthroscopies. Acta Chir Orthop Traumatol Cech 2008;75(5):382-6.

10. Bedi A, Feeley BT, Williams 3rd RJ. Management of articular cartilage defects of the knee. J Bone Joint Surg Am 2010;92(4):994-1009.

11. Mikos AG, Herring SW, Ochareon P, Elisseeff J, Lu HH, Kandel $\mathrm{R}$, et al. Engineering complex tissues. Tissue Eng 2006;12(12):3307-39.

12. Franke O, Durst K, Maier V, Goken M, Birkholz T, Schneider $\mathrm{H}$, et al. Mechanical properties of hyaline and repair cartilage studied by nanoindentation. Acta Biomater 2007;3(6):873-81.

13. Widuchowski W, Widuchowski J, Trzaska T. Articular cartilage defects: study of 25,124 knee arthroscopies. Knee 2007;14(3):177-82.

14. Williams SK, Amiel D, Ball ST, Allen RT, Wong VW, Chen AC, et al. Prolonged storage effects on the articular cartilage of fresh human osteochondral allografts. J Bone Joint Surg Am 2003;85-A(11):2111-20.

15. Gross AE, Kim W, Las HF, Backstein D, Safir O, Pritzker KP. Fresh osteochondral allografts for posttraumatic knee defects: long-term followup. Clin Orthopaedics Relat Res 2008;466(8):1863-70.

16. Koh JL, Wirsing K, Lautenschlager E, Zhang LO. The effect of graft height mismatch on contact pressure following osteochondral grafting: a biomechanical study. Am J Sports Med 2004;32(2):317-20.

17. Grande DA, Pitman MI, Peterson L, Menche D, Klein M. The repair of experimentally produced defects in rabbit articular cartilage by autologous chondrocyte transplantation. J Orthop Res 1989;7(2):208-18.

18. Brittberg M, Lindahl A, Nilsson A, Ohlsson C, Isaksson O, Peterson L. Treatment of deep cartilage defects in the knee with autologous chondrocyte transplantation. N Engl J Med 1994;331(14):889-95.

19. Vanlauwe J, Saris DB, Victor J, Almqvist KF, Bellemans J, Luyten FP, et al. Five-year outcome of characterized chondrocyte implantation versus microfracture for symptomatic cartilage defects of the knee: early treatment matters. Am J Sports Med 2011;39(12):2566-74.

20. Knutsen G, Drogset JO, Engebretsen L, Grontvedt T, Isaksen V, Ludvigsen TC, et al. A randomized trial comparing autologous chondrocyte implantation with microfracture. Findings at five years. J Bone Joint Surg Am 2007;89(10):2105-12.

21. Peterson L, Vasiliadis HS, Brittberg M, Lindahl A. Autologous chondrocyte implantation: a long-term follow-up. Am J Sports Med 2010;38(6):1117-24.

22. Harris JD, Siston RA, Brophy RH, Lattermann C, Carey JL, Flanigan DC. Failures, re-operations, and complications after autologous chondrocyte implantation-a systematic review. Osteoarthritis Cartilage 2011;19(7):779-91.

23. Dorotka R, Bindreiter U, Macfelda K, Windberger U, Nehrer S. Marrow stimulation and chondrocyte transplantation using a collagen matrix for cartilage repair. Osteoarthritis Cartilage 2005;13(8):655-64.

24. Breinan HA, Martin SD, Hsu HP, Spector M. Healing of canine articular cartilage defects treated with microfracture, a typeII collagen matrix, or cultured autologous chondrocytes. J Orthop Res 2000;18(5):781-9. 
25. Ahmed N, Gan L, Nagy A, Zheng J, Wang C, Kandel RA. Cartilage tissue formation using redifferentiated passaged chondrocytes in vitro. Tissue Eng 2009:665-73. Part(3).

26. Ahmed N, Taylor DW, Wunder J, Nagy A, Gross AE, Kandel RA. Passaged human chondrocytes accumulate extracellular matrix when induced by bovine chondrocytes. J Tissue Eng Regenerative Med 2010;4(3):233-41.

27. Albrecht FH. Closure of joint cartilage defects using cartilage fragments and fibrin glue. Fortschr Med 1983;101(37): $1650-2$.

28. Lu Y, Dhanaraj S, Wang Z, Bradley DM, Bowman SM, Cole BJ, et al. Minced cartilage without cell culture serves as an effective intraoperative cell source for cartilage repair. J Orthop Res 2006;24(6):1261-70.

29. Farr J, Cole BJ, Sherman S, Karas V. Particulated articular cartilage: CAIS and DeNovo NT. J Knee Surg 2012;25(1):23-9.

30. Hwang NS, Varghese S, Puleo C, Zhang Z, Elisseeff J. Morphogenetic signals from chondrocytes promote chondrogenic and osteogenic differentiation of mesenchymal stem cells. J Cell Physiol 2007;212(2):281-4.

31. Dell'Accio F, De BC, Luyten FP. Microenvironment and phenotypic stability specify tissue formation by human articular cartilage-derived cells in vivo. Exp Cell Res 2003;287(1):16-27.

32. Hettrich CM, Crawford D, Rodeo SA. Cartilage repair: thirdgeneration cell-based technologies-basic science, surgical techniques, clinical outcomes. Sports Med Arthrosc Rev 2008;16(4):230-5.

33. Kessler MW, Ackerman G, Dines JS, Grande D. Emerging technologies and fourth generation issues in cartilage repair. Sports Med Arthrosc Rev 2008;16(4):246-54.

34. Pittenger MF, Mackay AM, Beck SC, Jaiswal RK, Douglas R, Mosca JD, et al. Multilineage potential of adult human mesenchymal stem cells. Science 1999;284(5411):143-7.

35. Dominici M, Le BK, Mueller I, Slaper-Cortenbach I, Marini F, Krause D, et al. Minimal criteria for defining multipotent mesenchymal stromal cells. The International Society for Cellular Therapy position statement. Cytotherapy 2006;8(4): 315-7.

36. Aicher WK, Buhring HJ, Hart M, Rolauffs B, Badke A, Klein G. Regeneration of cartilage and bone by defined subsets of mesenchymal stromal cells - potential and pitfalls. Adv Drug Deliv Rev 2011;63(4-5):342-51.

37. Battula VL, Bareiss PM, Treml S, Conrad S, Albert I, Hojak S, et al. Human placenta and bone marrow derived MSC cultured in serum-free, b-FGF-containing medium express cell surface frizzled-9 and SSEA-4 and give rise to multilineage differentiation. Differentiation 2007;75(4):279-91.

38. Sakaguchi Y, Sekiya I, Yagishita K, Muneta T. Comparison of human stem cells derived from various mesenchymal tissues: superiority of synovium as a cell source. Arthritis Rheum 2005;52(8):2521-9.

39. Shirasawa S, Sekiya I, Sakaguchi Y, Yagishita K, Ichinose S, Muneta T. In vitro chondrogenesis of human synoviumderived mesenchymal stem cells: optimal condition and comparison with bone marrow-derived cells. J Cell Biochem 2006;97(1):84-97.

40. Wakitani S, Okabe T, Horibe S, Mitsuoka T, Saito M, Koyama T, et al. Safety of autologous bone marrow-derived mesenchymal stem cell transplantation for cartilage repair in 41 patients with 45 joints followed for up to 11 years and 5 months. J Tissue Eng Regenerative Med 2011;5(2):146-50.

41. Battula VL, Treml S, Bareiss PM, Gieseke F, Roelofs H, de Zwart P, et al. Isolation of functionally distinct mesenchymal stem cell subsets using antibodies against CD56, CD271, and mesenchymal stem cell antigen-1. Haematologica 2009;94(2):173-84.

42. Nejadnik H, Hui JH, Feng Choong EP, Tai BC, Lee EH. Autologous bone marrow-derived mesenchymal stem cells versus autologous chondrocyte implantation: an observational cohort study. Am J Sports Med 2010;38(6):1110-6.

43. Wakitani S, Nawata M, Tensho K, Okabe T, Machida H, Ohgushi H. Repair of articular cartilage defects in the patellofemoral joint with autologous bone marrow mesenchymal cell transplantation: three case reports involving nine defects in five knees. J Tissue Eng Regenerative Med 2007;1(1): 74-9.

44. Thomson JA, Itskovitz-Eldor J, Shapiro SS, Waknitz MA, Swiergiel JJ, Marshall VS, et al. Embryonic stem cell lines derived from human blastocysts. Science 1998;282(5391): 1145-7.

45. Heng BC, Cao T, Lee EH. Directing stem cell differentiation into the chondrogenic lineage in vitro. Stem Cells 2004;22(7): 1152-67.

46. Hwang NS, Varghese S, Lee HJ, Zhang Z, Ye Z, Bae J, et al. In vivo commitment and functional tissue regeneration using human embryonic stem cell-derived mesenchymal cells. Proc Natl Acad Sci U S A 2008;105(52):20641-6.

47. Toh WS, Guo XM, Choo AB, Lu K, Lee EH, Cao T. Differentiation and enrichment of expandable chondrogenic cells from human embryonic stem cells in vitro. J Cell Mol Med 2009;13(9B):3570-90.

48. Koay EJ, Hoben GM, Athanasiou KA. Tissue engineering with chondrogenically differentiated human embryonic stem cells. Stem Cells 2007;25(9):2183-90.

49. Toh WS, Yang Z, Liu H, Heng BC, Lee EH, Cao T. Effects of culture conditions and bone morphogenetic protein 2 on extent of chondrogenesis from human embryonic stem cells. Stem Cells 2007;25(4):950-60.

50. Wakitani S, Aoki H, Harada Y, Sonobe M, Morita Y, Mu Y, et al. Embryonic stem cells form articular cartilage, not teratomas, in osteochondral defects of rat joints. Cell Transplant 2004;13(4):331-6.

51. Wakitani S, Takaoka K, Hattori T, Miyazawa N, Iwanaga T, Takeda S, et al. Embryonic stem cells injected into the mouse knee joint form teratomas and subsequently destroy the joint. Rheumatology 2003;42(1):162-5.

52. Nakajima M, Wakitani S, Harada Y, Tanigami A, Tomita N. In vivo mechanical condition plays an important role for appearance of cartilage tissue in ES cell transplanted joint. J Orthop Res 2008;26(1):10-7.

53. Toh WS, Lee EH, Guo XM, Chan JK, Yeow CH, Choo AB, et al. Cartilage repair using hyaluronan hydrogel-encapsulated human embryonic stem cell-derived chondrogenic cells. Biomaterials 2010;31(27):6968-80.

54. Bai HY, Chen GA, Mao GH, Song TR, Wang YX. Three step derivation of cartilage like tissue from human embryonic stem cells by 2D-3D sequential culture in vitro and further implantation in vivo on alginate/PLGA scaffolds. J Biomed Mater Res Part A 2010;94(2):539-46.

55. Takahashi K, Yamanaka S. Induction of pluripotent stem cells from mouse embryonic and adult fibroblast cultures by defined factors. Cell 2006;126(4):663-76.

56. Robinton DA, Daley GQ. The promise of induced pluripotent stem cells in research and therapy. Nature 2012;481(7381): 295-305.

57. Wei Y, Zeng W, Wan R, Wang J, Zhou Q, Qiu S, et al. Chondrogenic differentiation of induced pluripotent stem cells from osteoarthritic chondrocytes in alginate matrix. Eur Cells Mater 2012;23:1-12. 
58. Safran MR, Kim H, Zaffagnini S. The use of scaffolds in the management of articular cartilage injury. J Am Acad Orthopaedic Surgeons 2008;16(6):306-11.

59. El-Ayoubi R, DeGrandpre C, DiRaddo R, Yousefi AM, Lavigne P. Design and dynamic culture of 3D-scaffolds for cartilage tissue engineering. J Biomater Appl 2011;25(5): 429-44.

60. Toh WS, Lee EH, Cao T. Potential of human embryonic stem cells in cartilage tissue engineering and regenerative medicine. Stem Cell Rev 2011;7(3):544-59.

61. Marcacci M, Berruto M, Brocchetta D, Delcogliano A, Ghinelli D, Gobbi A, et al. Articular cartilage engineering with hyalograft C: 3-year clinical results. Clin Orthop Relat Res 2005;435:96-105.

62. Gille J, Behrens P, Volpi P, de Girolamo L, Reiss E, Zoch W, et al. Outcome of Autologous Matrix Induced Chondrogenesis (AMIC) in cartilage knee surgery: data of the AMIC Registry. Arch Orthop Trauma Surg 2013;133(1):87-93.

63. Crawford DC, Heveran CM, Cannon Jr WD, Foo LF, Potter HG. An autologous cartilage tissue implant NeoCart for treatment of grade III chondral injury to the distal femur: prospective clinical safety trial at 2 years. Am J Sports Med 2009;37(7): $1334-43$.

64. Haddo O, Mahroof S, Higgs D, David L, Pringle J, Bayliss M, et al. The use of chondrogide membrane in autologous chondrocyte implantation. Knee 2004;11(1):51-5.

65. Grigolo B, Lisignoli G, Piacentini A, Fiorini M, Gobbi P, Mazzotti G, et al. Evidence for redifferentiation of human chondrocytes grown on a hyaluronan-based biomaterial (HYAff 11): molecular, immunohistochemical and ultrastructural analysis. Biomaterials 2002;23(4):1187-95.

66. Spiller KL, Maher SA, Lowman AM. Hydrogels for the repair of articular cartilage defects. Tissue Eng Part B Rev 2011;17(4): 281-99.

67. Gomoll AH. Microfracture and augments. J Knee Surg 2012;25(1):9-15.

68. McNickle AG, Provencher MT, Cole BJ. Overview of existing cartilage repair technology. Sports Med Arthrosc Rev 2008;16(4):196-201.

69. Shive MS, Hoemann CD, Restrepo A, Hurtig MB, Duval N, Ranger P, et al. BST-CarGel: in situ chondroinduction for cartilage repair. Oper Tech Orthopaedics 2012;16:271-8.

70. Williams GM, Klisch SM, Sah RL. Bioengineering cartilage growth, maturation, and form. Pediatr Res 2008;63(5): 527-34.

71. Fortier LA, Barker JU, Strauss EJ, McCarrel TM, Cole BJ. The role of growth factors in cartilage repair. Clin Orthop Relat Res 2011;469(10):2706-15.

72. Jakob M, Demarteau O, Schafer D, Hintermann B, Dick W, Heberer M, et al. Specific growth factors during the expansion and redifferentiation of adult human articular chondrocytes enhance chondrogenesis and cartilaginous tissue formation in vitro. J Cell Biochem 2001;81(2):368-77.

73. Francioli SE, Martin I, Sie CP, Hagg R, Tommasini R, Candrian C, et al. Growth factors for clinical-scale expansion of human articular chondrocytes: relevance for automated bioreactor systems. Tissue Eng 2007;13(6):1227-34.

74. Kuroda R, Usas A, Kubo S, Corsi K, Peng H, Rose T, et al. Cartilage repair using bone morphogenetic protein 4 and muscle-derived stem cells. Arthritis Rheum 2006;54(2): 433-42.

75. Diekman BO, Rowland CR, Lennon DP, Caplan AI, Guilak F. Chondrogenesis of adult stem cells from adipose tissue and bone marrow: induction by growth factors and cartilagederived matrix. Tissue Eng 2010:523-33. Part(2).
76. Munirah S, Ruszymah BH, Samsudin OC, Badrul AH, Azmi B, Aminuddin BS. Autologous versus pooled human serum for articular chondrocyte growth. J Orthop Surg 2008;16(2):220-9.

77. Drengk A, Zapf A, Sturmer EK, Sturmer KM, Frosch KH. Influence of platelet-rich plasma on chondrogenic differentiation and proliferation of chondrocytes and mesenchymal stem cells. Cells Tissues Organs 2009;189(5):317-26.

78. Lin SS, Landesberg R, Chin HS, Lin J, Eisig SB, Lu HH. Controlled release of PRP-derived growth factors promotes osteogenic differentiation of human mesenchymal stem cells. Conf Proc Annu Int Conf IEEE Eng Med Biol Soc 2006;1:4358-61.

79. Clar C, Cummins E, McIntyre L, Thomas S, Lamb J, Bain L, et al. Clinical and cost-effectiveness of autologous chondrocyte implantation for cartilage defects in knee joints: systematic review and economic evaluation. Health Technol Assess 2005;9(47).

80. Vasiliadis HS, Wasiak J, Salanti G. Autologous chondrocyte implantation for the treatment of cartilage lesions of the knee: a systematic review of randomized studies. Knee Surg Sports Traumatol Arthrosc 2010;18(12):1645-55.

81. Vavken P, Samartzis D. Effectiveness of autologous chondrocyte implantation in cartilage repair of the knee: a systematic review of controlled trials. Osteoarthritis Cartilage 2010;18(6):857-63.

82. Harris JD, Siston RA, Pan X, Flanigan DC. Autologous chondrocyte implantation: a systematic review. J Bone Joint Surg Am 2010;92(12):2220-33.

83. Cole BJ, Farr J, Winalski CS, Hosea T, Richmond J, Mandelbaum B, et al. Outcomes after a single-stage procedure for cell-based cartilage repair: a prospective clinical safety trial with 2-year follow-up. Am J Sports Med 2011;39(6):1170-9.

84. James AW, Xu Y, Lee JK, Wang R, Longaker MT. Differential effects of TGF-beta1 and TGF-beta3 on chondrogenesis in posterofrontal cranial suture-derived mesenchymal cells in vitro. Plast Reconstr Surg 2009;123(1):31-43.

85. Zhang B, Yang S, Sun Z, Zhang Y, Xia T, Xu W, et al. Human mesenchymal stem cells induced by growth differentiation factor 5: an improved self-assembly tissue engineering method for cartilage repair. Tissue Eng Part C Methods 2011;17(12):1189-99.

86. Fan J, Gong Y, Ren L, Varshney RR, Cai D, Wang DA. In vitro engineered cartilage using synovium-derived mesenchymal stem cells with injectable gellan hydrogels. Acta Biomater 2010;6(3):1178-85.

87. Goldberg AJ, Lee DA, Bader DL, Bentley G. Autologous chondrocyte implantation. Culture in a TGF-beta-containing medium enhances the re-expression of a chondrocytic phenotype in passaged human chondrocytes in pellet culture. J Bone Joint Surg Br 2005;87(1):128-34.

88. Luyten FP, Chen P, Paralkar V, Reddi AH. Recombinant bone morphogenetic protein-4, transforming growth factor-beta 1 , and activin A enhance the cartilage phenotype of articular chondrocytes in vitro. Exp Cell Res 1994;210(2):224-9.

89. Sekiya I, Colter DC, Prockop DJ. BMP-6 enhances chondrogenesis in a subpopulation of human marrow stromal cells. Biochem Biophys Res Commun 2001;284(2):411-8.

90. Gooch KJ, Blunk T, Courter DL, Sieminski AL, VunjakNovakovic G, Freed LE. Bone morphogenetic proteins-2, -12, and -13 modulate in vitro development of engineered cartilage. Tissue Eng 2002;8(4):591-601.

91. Chaipinyo K, Oakes BW, van Damme MP. Effects of growth factors on cell proliferation and matrix synthesis of lowdensity, primary bovine chondrocytes cultured in collagen I gels. J Orthop Res 2002;20(5):1070-8. 
92. Elisseeff J, McIntosh W, Fu K, Blunk BT, Langer R. Controlledrelease of IGF-I and TGF-beta1 in a photopolymerizing hydrogel for cartilage tissue engineering. J Orthop Res 2001;19(6):1098-104.

93. Gaissmaier C, Koh JL, Weise K. Growth and differentiation factors for cartilage healing and repair. Injury 2008;39(Suppl 96).

94. Mueller MB, Blunk T, Appel B, Maschke A, Goepferich A, Zellner J, et al. Insulin is essential for in vitro chondrogenesis of mesenchymal progenitor cells and influences chondrogenesis in a dose-dependent manner. Int Orthopaedics 2013;37(1):153-8.

95. Cuevas P, Burgos J, Baird A. Basic fibroblast growth factor (FGF) promotes cartilage repair in vivo. Biochem Biophys Res Commun 1988;156(2):611-8.

96. Siebert CH, Miltner O, Weber M, Sopka S, Koch S, Niedhart C. Healing of osteochondral grafts in an ovine model under the influence of bFGF. Arthroscopy 2003;19(2):182-7.
97. Mandl EW, Jahr H, Koevoet JL, van Leeuwen JP, Weinans H, Verhaar JA, et al. Fibroblast growth factor-2 in serum-free medium is a potent mitogen and reduces dedifferentiation of human ear chondrocytes in monolayer culture. Matrix Biol 2004;23(4):231-41.

98. Li X, Ellman MB, Kroin JS, Chen D, Yan D, Mikecz K, et al. Species-specific biological effects of FGF-2 in articular cartilage: implication for distinct roles within the FGF receptor family. J Cell Biochem 2012;113(7):2532-42.

99. Inoue H, Kato Y, Iwamoto M, Hiraki Y, Sakuda M, Suzuki F. Stimulation of cartilage-matrix proteoglycan synthesis by morphologically transformed chondrocytes grown in the presence of fibroblast growth factor and transforming growth factor-beta. J Cell Physiol 1989;138(2):329-37.

100. Kato Y, Iwamoto M. Fibroblast growth factor is an inhibitor of chondrocyte terminal differentiation. J Biol Chem 1990;265(10):5903-9. 


\title{
A combined additive manufacturing and micro-syringe deposition technique for realization of bio-ceramic structures with micro-scale channels
}

\author{
Mihaela Vlasea • Yaser Shanjani • Annabel Bothe • \\ Rita Kandel • Ehsan Toyserkani
}

Received: 15 April 2012 / Accepted: 14 February 2013 / Published online: 26 February 2013

(C) Springer-Verlag London 2013

\begin{abstract}
This article presents a novel rapid layered manufacturing approach based on a combined additive manufacturing (AM) process and a UV-based microsyringe deposition $(\mu \mathrm{SD})$ technique to be used in the fabrication of bio-ceramic structures with controlled micro-sized channels for bone and osteochondral tissue regeneration. In the proposed rapid manufacturing method, micro-scale sacrificial photopolymer networks are integrated within the manufactured part by depositing the photopolymer on selected bio-ceramic powder layers using an injection system. This AM- $\mu \mathrm{SD}$ method along with a post-processing protocol can potentially overcome current limitations of traditional powderbased AM approaches that are restricted in terms of complexity of internal architecture and feature size. For bone or osteochondral repair applications, the material system composed of the bio-ceramic and sacrificial photopolymer, along with the post-processing protocol, must ensure that the final implants are free from manufacturing residuals that could trigger an immune response post-implantation. In this study,
\end{abstract}

M. Vlasea $\cdot$ E. Toyserkani $(\bowtie)$

Department of Mechanical and Mechatronics Engineering,

University of Waterloo, 200 University Ave. West,

Waterloo, ON N2L 3G1, Canada

e-mail: etoyserk@uwaterloo.ca

Y. Shanjani

Department of Orthopedic Surgery, Stanford University,

Stanford, CA 94305, USA

\section{A. Bothe}

Hamburg University of Technology, Schwarzenbergstrasse 95, Hamburg 21073, Germany

R. Kandel

CIHR-Bioengineering of Skeletal Tissues Team, Mount Sinai Hospital, Toronto, ON M5G1X5, Canada calcium polyphosphate bio-ceramic was used as the substrate material based on prior art, polyvinyl alcohol solution was used as the powder binding agent, and ethoxylated (10 bisphenol A diacrylate) photopolymer solution was used as the sacrificial photopolymer element. Material characterization suggests that the proposed material system along with heat treatment protocol is suitable for the targeted applications where micro-scale channels within the implant are produced by $\mathrm{AM}-\mu \mathrm{SD}$.

Keywords Additive manufacturing · Micro-syringe deposition $\cdot$ Calcium polyphosphate $\cdot$ Sacrificial photopolymer $\cdot$ Bio-ceramic

\section{Introduction}

Musculoskeletal diseases involve a variety of conditions such as arthritis, osteoporosis, traumatic musculoskeletal injuries, spinal injuries, and spinal deformities [1]. Although they are mostly non-life-threatening, these conditions can become debilitating, diminishing the quality of life of the affected individual by causing ongoing pain, discomfort, inflammation, and restrictions in range of motion [1]. Aside from clinical repercussions, musculoskeletal conditions represent a major financial burden on the healthcare sector as the current treatment for advanced joint trauma is to fully or partially replace the damaged joint using tissue grafts or artificial prosthetics to restore near-normal functions of the articulating surfaces [1, 2]. There is a pressing need for a more successful approach to joint replacement and joint reconstruction procedures. One such approach focuses on tissue-engineered biocompatible and bioresorbable implants that can replace the damaged area of the joint, gradually mature, and seamlessly integrate with the native tissue [3]. The idea is to manufacture an implant that 
temporarily provides mechanical support and acts as a template for new bone tissue formation. The design of such an implant has to consider the internal architecture of bone, which entails interconnected porosity as well as complex networks of channels. Figure 1 illustrates features of natural bone translated into a matrix of micro-sized channels and interconnected pores that could be integrated throughout the volume of the implant. The desired range of interconnected pore or channel size varies between 100 and $500 \mu \mathrm{m}$ [4-6]. The organized networks of micro-channels facilitate cell attachment, encourage bone in-growth, and promote metabolic exchange and implant fixation $[7,8]$. The channels are also used to mimic the natural bone porous macro architecture evolved as an adaptation to mechanical loadings [9].

Extensive research has been conducted on appropriate methodologies for controlling the internal architecture and mechanical properties of bio-ceramic scaffolds for bone osteochondral tissue replacement and augmentation. The most pressing challenge in manufacturing these biomimetric scaffolds is the design and integration of internal porous structures such as interconnected channels. To this effect, Woesz et al. [10] employed an indirect AM approach, where the first step was to manufacture a photopolymer mold using stereolithography. The mold can be subsequently filled with the calcium phosphate bio-ceramic slurry. The material system was then exposed to a heat treatment protocol to disintegrate the mold and sinter the bio-ceramic, resulting in a part with the designed features in the range of 300 to $500 \mu \mathrm{m}$. Manjubala et al. [11] investigated another indirect approach, where a wax mold was produced using a $3 \mathrm{D}$ printing approach. A chitosanhydroxyapatite solution was used to fill the mold and freezedried. The mold was then chemically disintegrated by exposure to an alcoholic sodium hydroxide solution, resulting in features of approximately $500 \mu \mathrm{m}$ and $50 \%$ porosity. An indirect approach was also used by Lee et al. [12] in fabricating poly(D,L-lactic-co-glycolic acid) scaffolds by making use of a plaster mold and sucrose porogens. They succeeded in making channels with a feature diameter averaging $700 \mu \mathrm{m}$. These techniques involve multiple and complex manufacturing steps as they are indirect approaches. They also generally require a range of harsh solvents to remove the molds or sacrificial elements. One of the most pressing limitations of these methods is that they are not proficient in having direct control over the internal feature characteristics of the scaffold as they often employ randomly distributed sacrificial porogens salts, or they rely on the accuracy of the mold and on proper infiltration of the slurry within the mold.

Powder-based additive manufacturing (AM) approaches via three-dimensional printing show promise in the field of tissue engineering and more specifically for bone and osteochondral implant fabrication $[4,13]$. Powder-based AM approaches, however, are currently restricted in terms of size of implants with interconnected channel features that can be produced. For larger implants with complex interconnected internal features, it is very difficult to remove trapped residual material from inside the implant structure; therefore, porosity and internal channel configuration cannot be properly controlled; the current capabilities of powder-based AM techniques cannot reliably allow for features below $500 \mu \mathrm{m}$ in size and complex channels [13]. These limitations need to be surpassed in order to produce large-scale anatomically shaped implants with controlled densities, micro- and macroporosities, and interconnected networks. The aforementioned implant fabrication limitations can be overcome by combining the capabilities of powder-based AM techniques with a microsyringe deposition $(\mu \mathrm{SD})$ process, which has not been reported previously. Using such a hybrid technique, a sacrificial element can be injected over selected powder layers during the $3 \mathrm{D}$ printing process. This manufacturing process requires a post-processing heat treatment procedure used to concomitantly sinter the structures and to remove the sacrificial polymeric element in order to eventually form micro-scale networks of channels within the part. The appropriate polymer is not only injectable via a micro-syringe system but is also a material which leaves no harmful residuals after the post-heat treatment process. This proposed novel fabrication method can be considered to be one of the more appropriate techniques for fabricating ceramic structures where a refined
Fig. 1 Natural bone morphology and a potential network of channels and/or macro-pores that can be integrated in the implant structure to mimic natural bone architecture
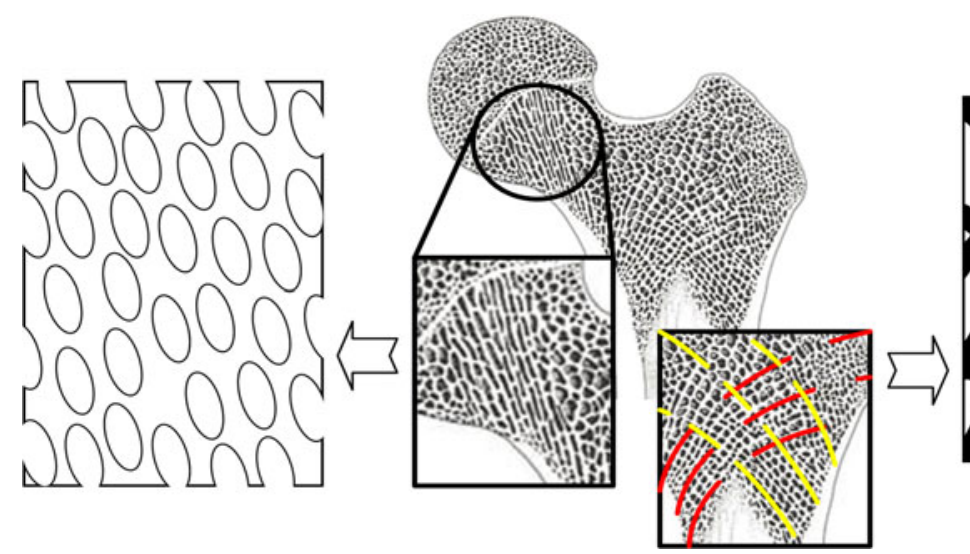

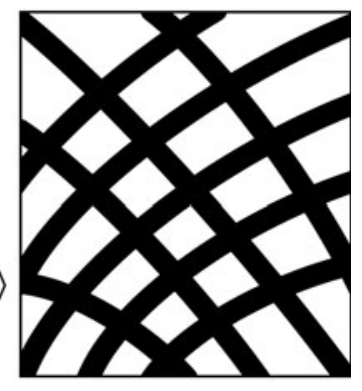


feature size and controlled internal part complexity are important criteria.

This work investigates the feasibility of producing bioceramic structures with embedded micro-channels using the proposed novel AM- $\mathrm{SSD}$ technique. The main purpose of this study is to demonstrate that the proposed sacrificial photopolymer can be deposited onto and embedded into powder substrates using the AM- $\mu \mathrm{SD}$ technique and that the required heat treatment protocol ensures that the photopolymer is fully removed during the implant post-processing stage to prevent host immune responses or other detrimental side effects postimplantation. In this work, this is achieved by selecting a bioceramic with known biocompatibility and determining if the $\mathrm{AM}-\mu \mathrm{SD}$ process is a feasible manufacturing approach, as well as if the introduction of the photopolymer changes the final chemical and crystalline properties of the part.

\section{Materials and methods}

\subsection{Structure material}

In this study, calcium polyphosphate (CPP) powder with batch sizes of 40-45 and 75-100 $\mu \mathrm{m}$ was used as the implant material along with polyvinyl alcohol (PVA) 4 wt.\% solution in deionized water as a binder. The binder was delivered by a peizobased print-head (XAAR, 1001 model, Xaar, Cambridge, UK).

CPP is amongst one of the more promising materials for producing structures for bone replacement. Calcium polyphosphate is a form of condensed calcium phosphate with a lower ratio of $\mathrm{Ca} / \mathrm{P}$ that forms linear polymeric-like phosphate chains [14]. This material is biocompatible and biodegradable, forming calcium orthophosphate, which is a "naturally occurring and readily metabolizable substance" [15]. The material is also osteoconductive, allowing rapid bone in-growth into the implant [16] as suggested by in vitro [14] and in vivo [17] studies. Recent investigations show that depending on the CPP powder particle size, the pore interconnectivity was moderate and that the compression strength shows promise for bone tissue engineering $[14,15]$.

\subsection{Photopolymer material and preparation}

The proposed photopolymer solution is comprised of a monomer, a reactive dilutant mixed to achieve the desired injectability, and a photoinitiator (PI) to control the UV light sensitivity. Ethoxylated (10 bisphenol A diacrylate) (EBA) (Ebecryl 150, Cytec, NJ, USA) was used as a monomer. Cellulose acetate butyrate (CAB) (Sigma Aldrich, Oakville, Canada) was dissolved in acetone and functions as dilutant. Phenylbis(2,4,6-trimethylbenzoyl)phosphine oxide (Irgacure 819) (Sigma Aldrich, Oakville, Canada) was utilized as a PI. The components were utilized as received and mixed in solution at room temperature with a composition of $13 \mathrm{wt} . \%$ CAB, 56 wt.\% EBA, 30.5 wt.\% acetone, and $0.5 \%$ PI. A PI concentration of $0.5 \mathrm{wt} \%$ resulted in an acceptable photopolymerization time of approximately $3 \mathrm{~s}$ when exposed to UV light (RX FireFly 25x10AC395, Phoseon Technology, Hillsboro, OR, USA) radiated from a maximum distance of $10 \mathrm{~cm}$. For the sake of simplicity, in this study, the solution is referred to as $\mathrm{EBA}^{*}$.

\subsection{Fabrication process}

\subsubsection{Additive manufacturing process description}

The initial stage of the AM- $\mu \mathrm{SD}$ process is to design a three-dimensional computer-aided design (CAD) model based on the desired bio-structures while considering the capabilities of the system and the morphological properties of the natural bone section to be replaced [13]. The details of this are beyond the scope of this paper.

The second stage in the AM- $-\mu \mathrm{SD}$ process is the actual fabrication of the implant. The combined AM- $\mu$ SD technique employs CPP ceramic powder as the substrate material. The structure is built in a layer-by-layer fashion in multiple steps as shown in Fig. 2. Within each layer, the powder is first spread using a counter-rotating roller mechanism and then bonded together at specific locations by injecting a binder using an inkjet-like printing technology. A new layer of powder is subsequently spread on top. The cycle is repeated until the part is completed. On specific layers, the photopolymer is injected using a micro-syringe mechanism. The novel AM- $\mu$ SD system for such a process has been developed inhouse. Figure 3 depicts a view of this system.

\subsubsection{Sacrificial photopolymer deposition process description}

The photopolymer was injected using a custom $\mu \mathrm{SD}$ system under an intermittent UV light (Phoseon Technology, FireFly 25x10, OR, USA) exposure from a distance of $1 \mathrm{~cm}$. Different injection process parameters were tested, which will be discussed in a separate article. The injection head velocity varied from 1 to $2.5 \mathrm{~mm} / \mathrm{s}$, and the value of the flow rate was between 2.5 and $10 \mu \mathrm{L} / \mathrm{min}$. A nozzle size of $150 \mu \mathrm{m}$ was used. The proposed photopolymer achieved consistent and accurate deposition tracks, proving that this photopolymer was a feasible choice in terms of injectability and curing time. Figure 3 shows a typical network of photopolymer on a powder substrate.

2.4 Post-process - binder removal, photopolymer removal, and sintering

The completed parts were left overnight and exposed to a heat treatment to fully cure or anneal the green part. Samples 
Fig. 2 Schematic of the novel AM- $\mu$ SD fabrication process for production of entire implants in a layer-by-layer fashion through sequential steps 1 to 4

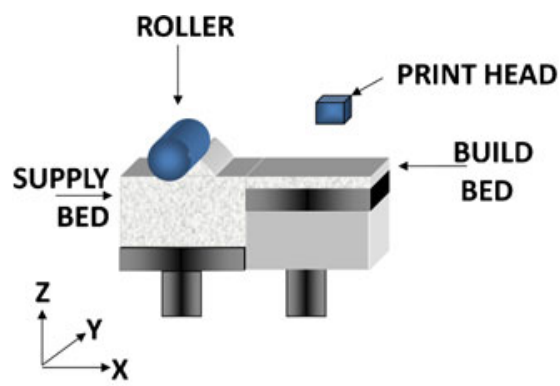

Step1:

Spread new powder layer using a custom roller mechanism.
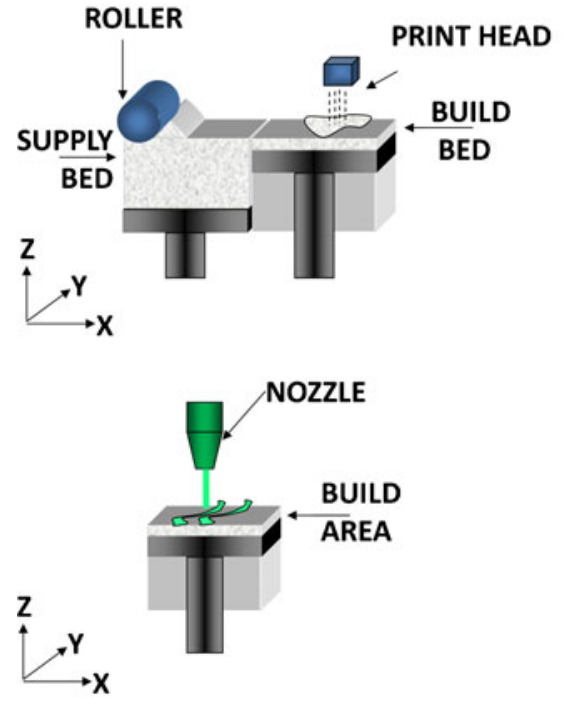

Step 3:

Extrude photopolymer through a nozzle, based on layer specifications
Step 2:

Inject binder using print-head based on CAD layer data

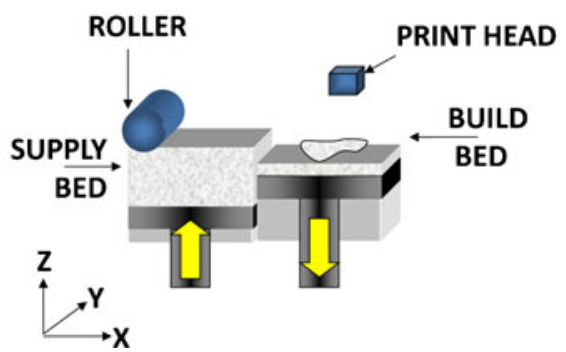

\section{Step 4:}

Lower build bed, raise supply bed, deposit new powder layer were air-annealed in a high-temperature furnace (EQ-GSL1500X-40, MTI Corporation, Richmond, CA, USA) with an established heat treatment protocol [18]. The heating rate was $10{ }^{\circ} \mathrm{C} / \mathrm{min}$ from room temperature to $400{ }^{\circ} \mathrm{C}$, holding for $1 \mathrm{~h}$, followed by another heating cycle of $10^{\circ} \mathrm{C} / \mathrm{min}$ up to $600{ }^{\circ} \mathrm{C}$ and a dwell time of $1 \mathrm{~h}$, ending with another
Fig. 3 In-house-developed AM- $\mu$ SD fabrication system with a close-up view of a typical manufactured layer presenting the deposited photopolymer structure on the powder substrate

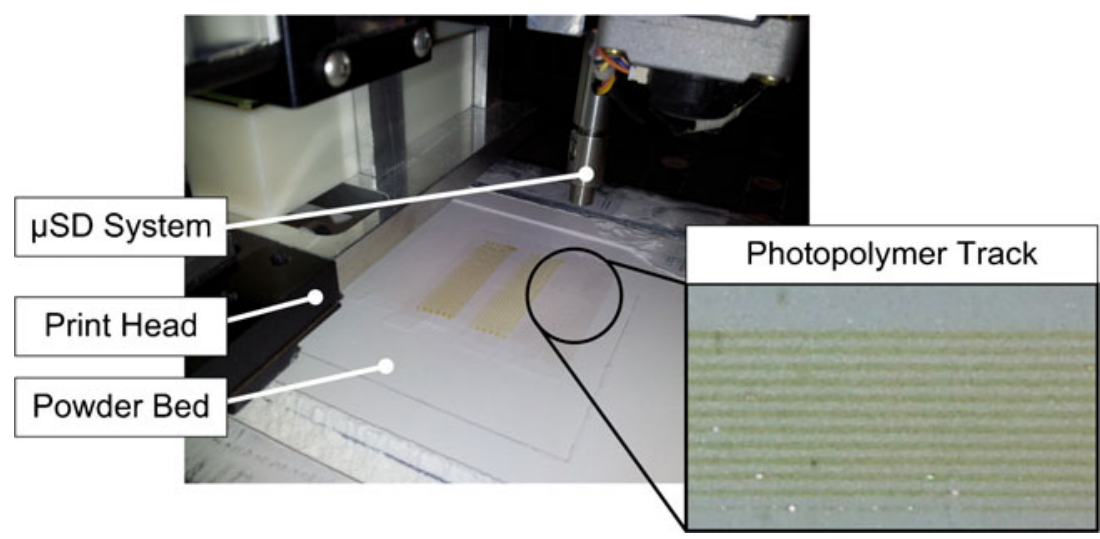


heating cycle of $10{ }^{\circ} \mathrm{C} / \mathrm{min}$ up to $950{ }^{\circ} \mathrm{C}$ with a dwell time of $1 \mathrm{~h}$ to ensure the formation of crystalline CPP and vaporization of any residual carbon from the structures. The cooling process was controlled for $4 \mathrm{~h}$.

\subsection{Sample preparation and characterization}

Samples were manufactured using the methodology explained in the last section. To this end, 25 layers $(100 \mu \mathrm{m}$ in thickness, $50 \times 50 \mathrm{~mm}^{2}$ area) of CPP was first spread and bonded. Subsequently, the $\mu \mathrm{SD}$ system was used to deposit four tracks of photopolymer (sample A, $4.5 \mathrm{~mm}$ in length, $2 \mathrm{~mm}$ apart) for optical microscopy and (sample B, $20 \mathrm{~mm}$ in length, $2 \mathrm{~mm}$ apart) intended for scanning electron microscopy. The tracks were covered with another ten layers of CPP as previously described. The part was sintered using the established postprocess heat treatment protocol.

\subsection{Geometrical properties of formed channels}

To ensure the formation of channels after the heat treatment protocol, a sample (sample A) with integrated channels was embedded in epoxy, dissected in vertical plane using a band saw, and polished to observe the formation of channels under an optical microscope (Olympus Japan BH Optical Microscope, Carsen Medical \& Scientific Co. Ltd., Markham, Ontario, Canada). In addition, to better observe the quality of the formed channels after the heat treatment protocol, another sample (sample B) with integrated channels was dissected in the longitudinal plane and observed using a scanning electron microscope (SEM; JSM-6460, Jeol, Akishima, Tokyo) at $20 \mathrm{kV}$ accelerating voltage. To make the sample electrically conductive, they were sputter-coated with a 10-nm gold layer (Desk II, Denton Vacuum, LCC, Moorestown, NJ, USA).

\subsection{Polymer thermal decomposition properties}

One of the initial steps in validating the AM- $\mu$ SD methodology was to establish the thermal degradation pathway and degradation temperature profile of the photopolymer using differential scanning calorimetry (DSC)-thermo gravimetric analysis (TGA) (SDT Q600, TA Instruments, Grimsby, Canada) under the same heat treatment protocol used for curing the samples. The EBA* photopolymer solution was injected through our micro-syringe and UV-cured to form a small drop weighing $10 \mathrm{mg}$. The same experimental analysis was performed on $10 \mathrm{mg}$ of pure PVA polymer to ensure that this polymer also fully degrade during the heat treatment protocol.

\subsection{Surface chemical composition}

For investigating the surface chemical composition of CPP structures after sintering, two types of heat-treated samples were prepared: a CPP-PVA baseline implant and a CPPPVA-EBA* implant containing the photopolymer. The chemical composition after sintering of the CPP-PVA and CPP-PVA-EBA* samples was analyzed using a field emission scanning electron microscope (FESEM) with energydispersive X-ray spectroscopy (EDAX) (LEO1530, Carl Zeiss AG., Peabody, MA, USA). Due to the nonconductivity of samples, 10 -nm gold layer coating was deposited using a sputter coater (Desk II, Denton Vacuum, LCC, Moorestown, NJ, USA) prior to FESEM-EDAX analysis. Both samples were investigated at a magnification of $\times 350$. Two investigation areas were the focus of each sample, 250 and $50 \mu^{2}$.

\subsection{Surface material composition}

$\mathrm{X}$-ray diffraction (XRD) was used to examine the material composition and crystallinity of the heat-treated CPP-PVA and CPP-PVA-EBA* samples to establish if there are any significant differences introduced through the presence of the photopolymer during the fabrication process. The XRD analysis equipment (RAPID II X-ray Detector, Rigaku, TX, USA) operated with $50 \mathrm{kV}$ and $40 \mathrm{~mA}$. The exposure time was $15 \mathrm{~s}$, and the angle of interest was selected between $15^{\circ}$ and $65^{\circ}$.

\section{Results}

\subsection{Geometrical properties of formed channels}

As shown in Fig. 4, the combined AM- $\mathrm{SSD}$ process resulted in the creation of channels inside CPP porous implants, which was one of the goals of this study. The SEM images depicted in Fig. 5 show that, qualitatively, the tracks produced using the AM- $\mu \mathrm{SD}$ method are visible, well defined and consistent. Some channels show variability in terms of channel width (Fig. 5a) from approximately 275 to $225 \mu \mathrm{m}$, while the channel in Fig. $5 \mathrm{~b}$ is relatively constant in width at around $260 \mu \mathrm{m}$. The channels were originally spaced $2 \mathrm{~mm}$ apart; however, due to the part shrinkage during the post-processing heat treatment, the distance between channels was reduced by $\sim 12.5 \%$.

\subsection{Polymer thermal decomposition properties}

DSC-TGA was performed on the EBA* and PVA, respectively, to determine the change in weight of the sample as a function of increasing temperature and eventually identify temperatures that are necessary to initiate thermal degradation and also the maximum temperature necessary to complete the decomposition process. The resulting DSC-TGA analysis for the photopolymer is illustrated in Fig. 6a. As can be seen, decomposition becomes significant after $300^{\circ} \mathrm{C}$, and it reveals 
Fig. 4 Cross-section of CPP part with integrated channels after heat treatment
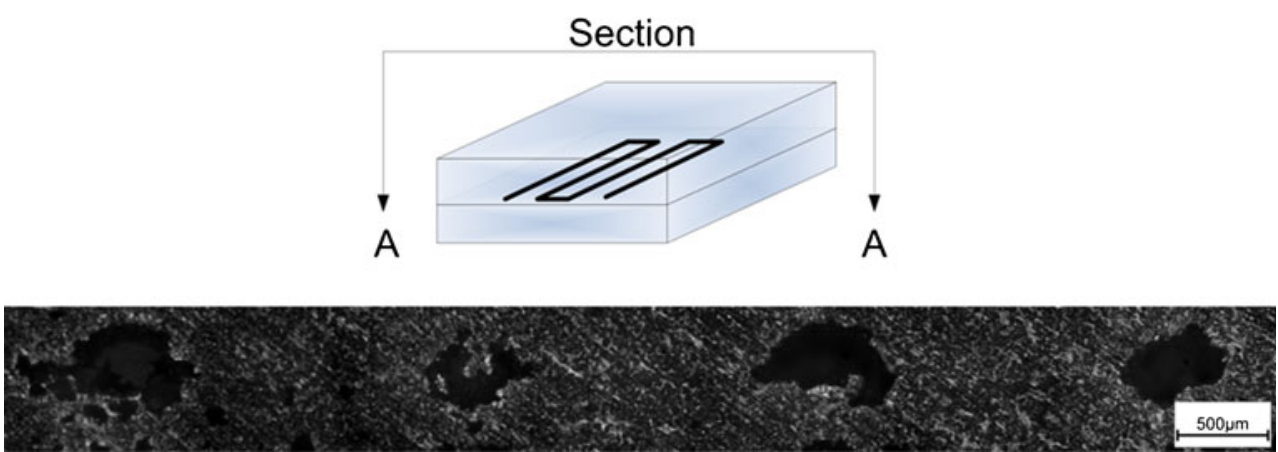

one degradation region for $\mathrm{EBA}^{*}$ at $\sim 450{ }^{\circ} \mathrm{C}$. The resulting DSC-TGA analysis for the PVA sample is illustrated in Fig. 6b. As observed, the degradation process becomes significant after $200{ }^{\circ} \mathrm{C}$, and it peaks in two regions at 300-325 and $400-425^{\circ} \mathrm{C}$, where the mass loss rate is accelerated. After $500{ }^{\circ} \mathrm{C}$, both photopolymer and PVA have been completely decomposed at a temperature significantly below the maximum sintering temperature of $\mathrm{CPP}\left(950^{\circ} \mathrm{C}\right)$.

\subsection{Surface chemical composition}

Figure 7 illustrates a typical result obtained through the FESEM-EDAX analysis. Table 1 summarizes the chemical composition of each sample in terms of weight percentage (wt.\%). The elements calcium $(\mathrm{Ca})$, oxygen $(\mathrm{O})$, phosphorus $(\mathrm{P})$, and carbon $(\mathrm{C})$ were present in both CPP-PVA and CPP-PVA-EBA* structures. Based on this analysis, it is
Fig. 5 CPP part with integrated micro-channels after heat treatment: $\mathbf{a}, \mathbf{b}$ top view and $\mathbf{c}$, d view from a $30^{\circ}$ inclination with different magnification

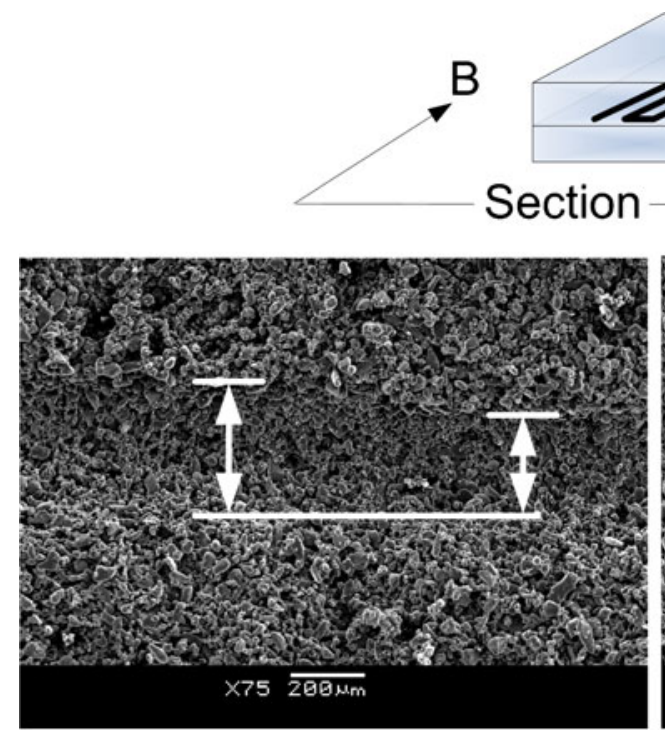

a)

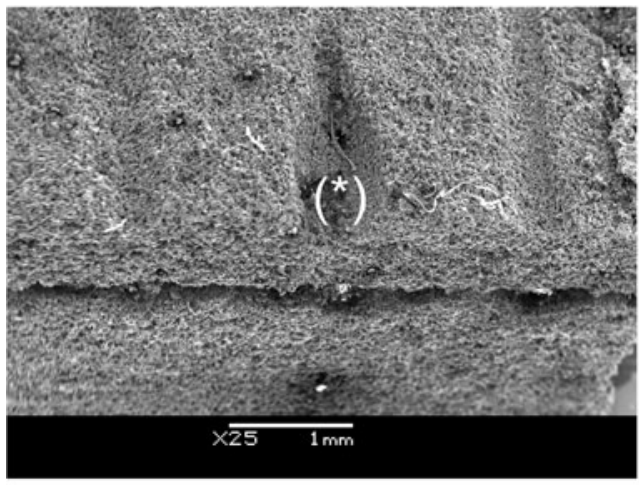

c)

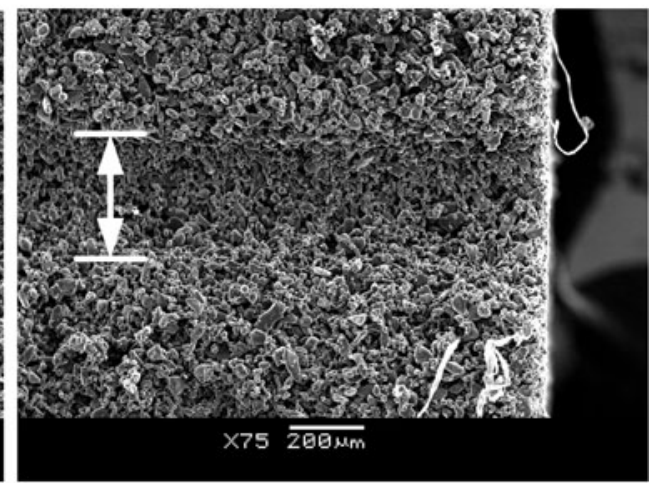

b)

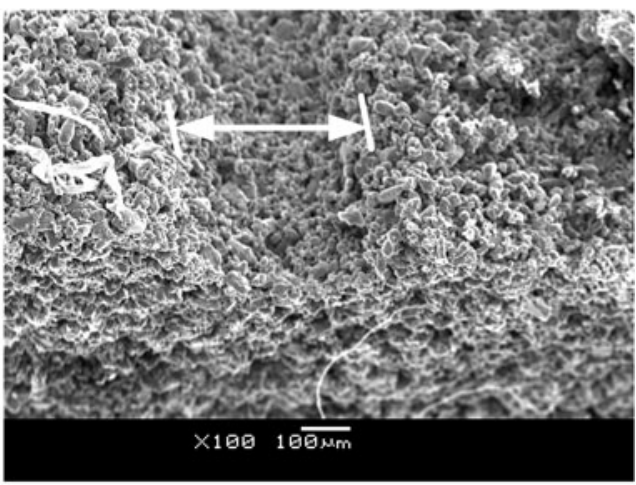

d) 


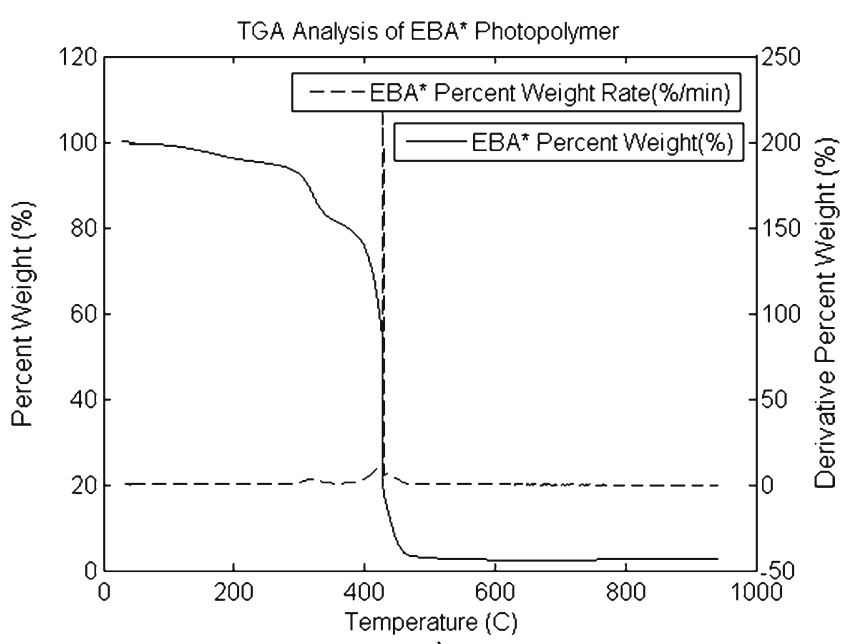

a)

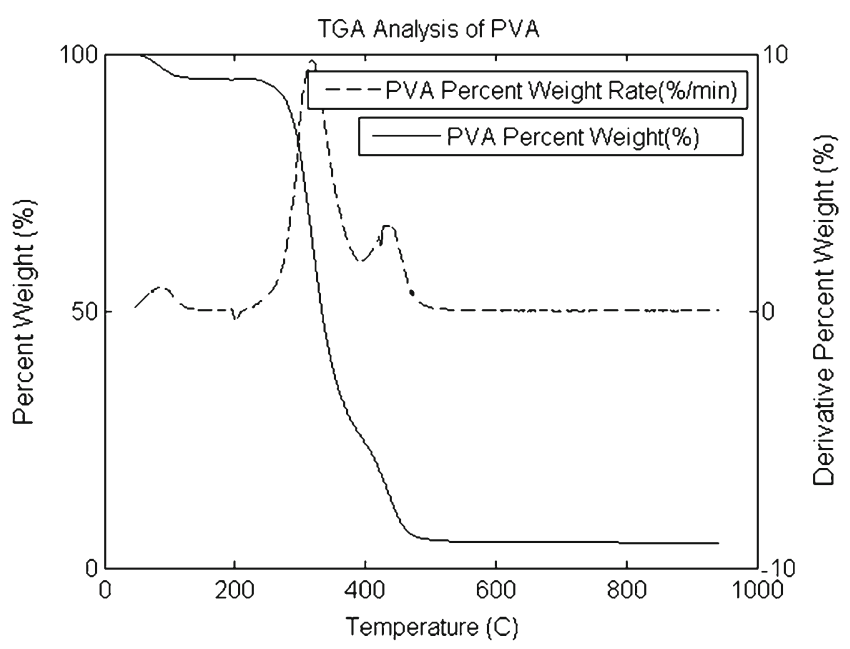

b)

Fig. 6 Polymer thermal decomposition TGA results of a EBA* and b PVA

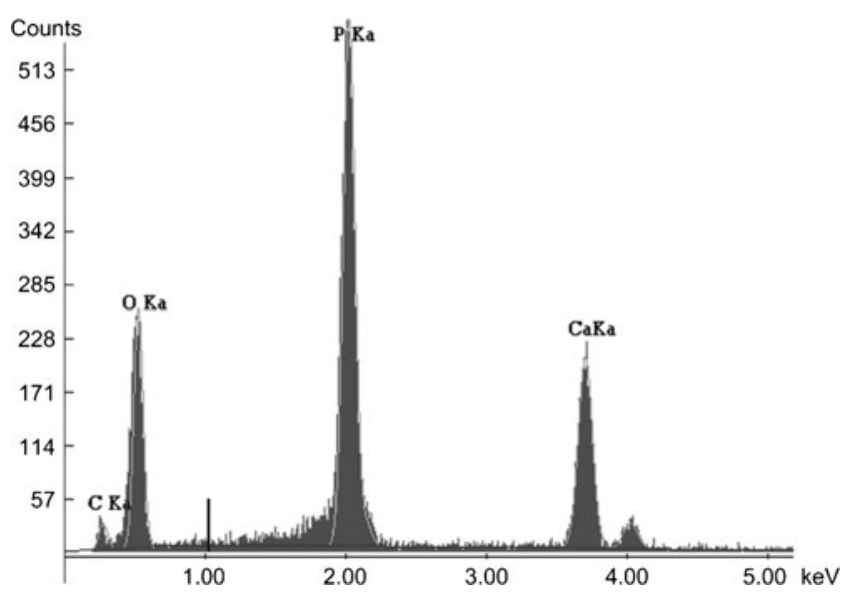

Fig. 7 FESEM-EDAX analysis of a typical CPP-PVA-EBA* sample using a $250-\mu \mathrm{m}^{2}$ area
Table 1 FESEM-EDAX analysis results

\begin{tabular}{llll}
\hline Trials & Elements & $\begin{array}{l}\text { CPP-PVA-EBA* } \\
\text { (wt \%) }\end{array}$ & $\begin{array}{l}\text { CPP-PVA } \\
\text { (wt \%) }\end{array}$ \\
\hline $\begin{array}{c}\text { Trial 1 250 } \mu \mathrm{m}^{2} \text { analysis } \\
\text { area for each sample }\end{array}$ & $\mathrm{C}$ & 14.63 & 13.94 \\
& $\mathrm{O}$ & 44.10 & 41.17 \\
& $\mathrm{P}$ & 26.11 & 27.01 \\
Trial 2 50 $\mathrm{m}^{2}$ analysis & $\mathrm{C}$ & 15.16 & 17.88 \\
area for each sample & $\mathrm{O}$ & 14.05 & 15.25 \\
& $\mathrm{P}$ & 26.56 & 36.86 \\
& $\mathrm{Ca}$ & 15.58 & 28.37 \\
& & & 19.52 \\
\hline
\end{tabular}

concluded that no additional chemical elements are present in the sample produced using the proposed photopolymer. The weight percentage for both trials 1 and 2 was very similar amongst samples. The discrepancies can be attributed to the small sampling size used to determine the results.

Figure 8 shows the XRD results indicating that there are no significant differences in peaks and intensities between the two types of samples analyzed. Based on these results, it can be inferred that there are no significant differences in chemical composition and crystallinity between the two samples and that the use of a photopolymer in the AM process does not introduce any by-products in the final product. The similarity of the XRD patterns and samples shown in our previous study [19] indicates that the resulting sintered CPP is $\beta$-CPP.

\section{Discussion}

A combined additive manufacturing and micro-syringe deposition technique was developed to achieve the formation of micro-channels within ceramic structures. This technique has the potential of integrating channels with complex geometry and orientation. The pattern of channels can be designed in a pre-process stage in a CAD environment. The design of the channels may be developed based upon the biological requirements of the final bio-structure and desired mechanical properties. The feature size of such channels has a direct effect on the performance of the implants. Based on biological functionality, the desirable channel feature should be in the range of $100-500 \mu \mathrm{m}$ in size [4-6]. To achieve nutrient transport and cell in-growth, the channels must be continuous and not clogged with the constitutive material of the structure. Any attempts towards forming very small features such as complex microchannels, pores, and holes embedded in parts using the available powder-based AM processes result in clogging since loose powder becomes trapped within the channels or pores [13]. The developed $\mathrm{AM}-\mu \mathrm{SD}$ technique addresses 
Fig. 8 XRD analysis of CPPPVA versus CPP-PVA-EBA* heat-treated samples
XRD Analysis of Sintered CPP-PVA and CPP-PVA-EVA* Samples

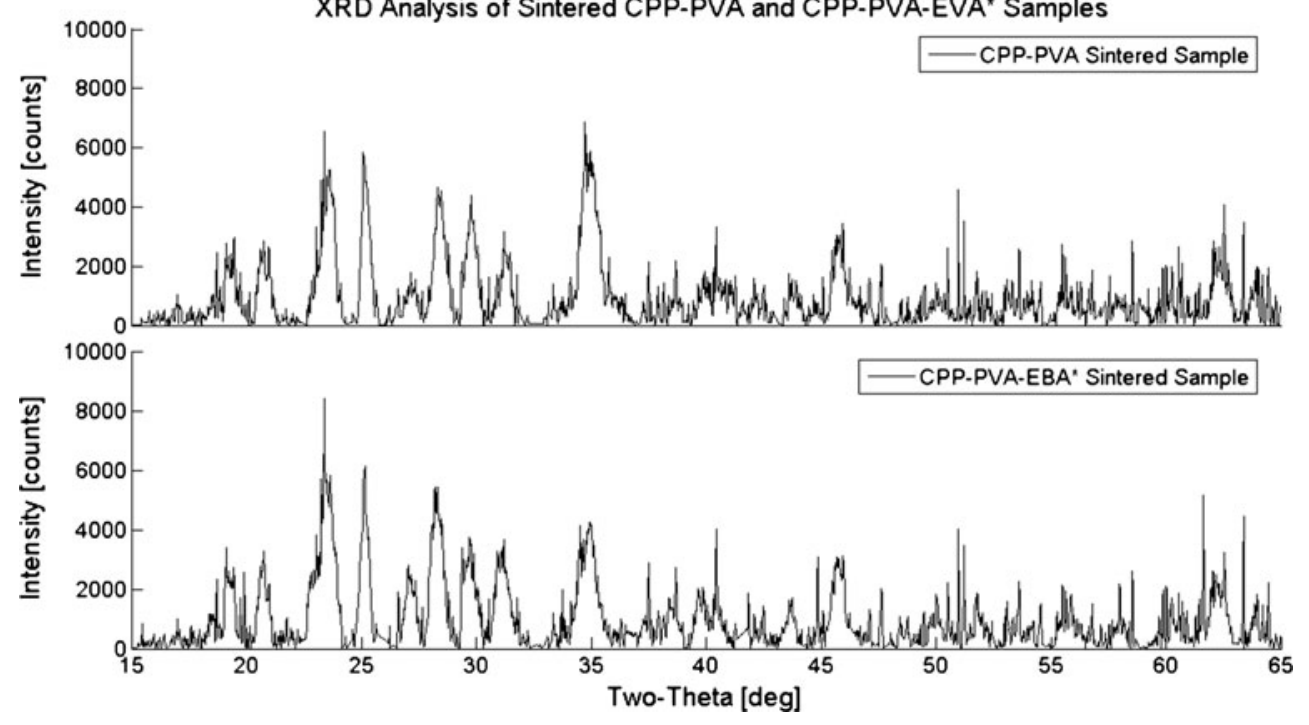

this concern as the resulting channels within CPP structures were continuous and include small features (as shown in Fig. 5). The results of these feasibility studies are reflective of samples produced without applying any optimizations, which is the focus of future work.

The cross-section shape of the channels depends on the geometry of injected/deposited photopolymer tracks. In addition, it should be noted that the post-processing stage determines the final geometry of the channels based on powder particle size and shape. The powder particle size may have a profound impact on the geometry of the channels as it affects the sintering shrinkage [20]. In addition, parts shrink during sintering when exposed to high temperature and, consequently, the geometrical feature of the channels change as CPP particles start to form sinter-necks and may rearrange after photopolymer burn-off. Consequently, the sintering protocol may affect the size and shape of the features [19]. Furthermore, CPP structures made via $\mathrm{AM}-\mu \mathrm{SD}$ are likely to shrink anisotropically if the shape of the particles has an aspect ratio larger than 1 [15]. The different shrinkage in the directions parallel and perpendicular to constitutive layers may contribute to the irregular shape of the channels and is subject to future work.

In addition, the deposition of the photopolymer tracks on the powder substrate is affected by the flatness of an underlying powder layer spread by the counter-rotating roller which, in turn, is drastically affected by the particle size. The finer the particle size is, the smoother the powder bed will be, which results in a more uniform photopolymer deposition. A future study will focus on optimizing the size and integrity of the channels by tuning the $\mu \mathrm{SD}$ injection parameters to reduce the feature size and the UV light exposure time and height to optimize the shape integrity.

Material characterization studies including TGA and XRD analyses also revealed that there was no residual by- product left from the photopolymer after the heat treatment stage. This matter is very crucial for evaluation of the developed method since any residual by-product material in the final part may adversely affect the biological properties. A comparison between the TGA analysis for the two polymers shown in Fig. 6 suggests that both followed the same degradation trend; however, the degradation of PVA occurred over a higher temperature range $\left(200\right.$ to $\left.450{ }^{\circ} \mathrm{C}\right)$, while EBA* was almost entirely degraded at around $450^{\circ} \mathrm{C}$. In both cases, the equilibrium weight does not stabilize to about 0 , which may be due to the system errors associated with initial calibration, as no visible residue was found after the heating sequence [21]. However, the degradation curves stabilize significantly below the highest curing temperature of $950{ }^{\circ} \mathrm{C}$, which is the topic of interest for this experiment analysis. In addition, XRD patterns of the CPP-PVA-EBA* and CPP-PVA samples confirm that the sacrificial photopolymer does not influence the crystalline structure of the post-processed part. It can be concluded that the material system was selected properly for the purpose of forming channels within bio-structures.

In the present study, the formation of micro-channels in planes parallel to the direction of layers was examined. The formation of channels with complex shapes and/or conformal configuration will be investigated in future work.

\section{Conclusions and future work}

This study introduced a novel combined additive manufacturing and micro-syringe deposition (AM- $\mu \mathrm{SD})$ process for manufacturing bio-ceramic structures with micro-sized channels for bone and osteochondral tissue regeneration. This methodology addresses current obstacles associated with powder-based additive manufacturing in terms of embedding 
complex channels with a small feature size within the internal architecture of parts. In this work, it was shown that these obstacles can be addressed using the newly developed $\mathrm{AM}-\mu \mathrm{SD}$ protocol by producing porous ceramic structures with embedded internal channels with features in the range of $\sim 200-500 \mu \mathrm{m}$ which are desirable for bone repair. A material system was proposed for this manufacturing technology to fulfill an important material design criterion, which is the prevention of any harmful residuals in the final product after the heat treatment protocol. The experimental analysis showed no signs of pyrolysis residuals left in the CPP structures, and no additional phase was observed in crystalline CPP material. As a result, the injectable photopolymer can effectively be used in a combined $\mathrm{AM}-\mu \mathrm{SD}$ process to act as a sacrificial element required for making complex micro-scale channels inside biodegradable ceramic structures. The aim of the future work is to optimize the photopolymer injection and deposition process on particulate substrates through the real-time control of the deposited photopolymer. Furthermore, a shrinkage analysis will be performed to establish a correlation between the geometrical characteristics of the photopolymer tracks and the resulting features.

Acknowledgments This work was funded by the Department of Defense US Army (Medical Research Acquisition Activity-grant number OR090169).

\section{References}

1. Hutchinson M (2009) The burden of musculoskeletal diseases in the United States: prevalance, societal and economic cost. J Am Coll Surg 208(1):e5-e6

2. Gikas PD, Bayliss L, Bentley G, Briggs TWR (2009) An overview of autologous chondrocyte implantation. Bone and Joint Surgery British Volume 91(8):997-1006

3. Hutmacher DW (2000) Scaffolds in tissue engineering bone and cartilage. Biomaterials 21(24):2529-2543

4. Yang S, Leong KF, Du Z, Chua CK (2001) The design of scaffolds for use in tissue engineering. Part I. Traditional factors. Tissue Eng 7(6):679-689

5. Chang BS, Lee CK, Hong KS, Youn HJ, Ryu HS, Chung SS, Park KW (2000) Osteoconduction at porous hydroxyapatite with various pore configurations. Biomaterials 21(12):1291-1298

6. Kujala S, Ryhanen J, Danilov A, Tuukkanen J (2003) Effect of porosity on the osteointegration and bone ingrowth of a weight- bearing nickel-titanium bone graft substitute. Biomaterials 24(25):4691-4697

7. Martin I, Miot S, Barbero A, Jakob M, Wendt D (2007) Osteochondral tissue engineering. Biomechanics 40(4):750-765

8. Kandel RA, Grynpas M, Pilliar R, Lee J, Wang J, Waldman S, Zalzal P, Hurtig M (2006) Repair of osteochondral defects with biphasic cartilage-calcium polyphosphate constructs in a sheep model. Biomaterials 27(22):4120-4131

9. Bartel DL, Davy DT, Keaveny TM (2009) Orthopaedic biomechanics - mechanics and design in musculoskeletal systems. Upper Saddle River, Pearson Prentice Hall, pp 110-115

10. Woesz A, Rumpler M, Stampf J, Varga F, Fratzl-zelman N, Roschger P (2005) Towards bone replacement materials from calcium phosphates via rapid prototyping and ceramic gel casting. Gene 25:181-186

11. Manjubala I, Woesz A, Pilz C, Rumpler M, Fratzl-zelman N, Roschger P, Stampfl J, Fratzl P (2005) Biomimetic mineral-organic composite scaffolds with controlled internal architecture. Materials Science: Materials in Medicine 6:1111-1119

12. Lee M, Dunn JCY, Wu BM (2005) Scaffold fabrication by indirect three-dimensional printing. Biomaterials 26(20):4281-4289

13. Leong K, Cheah C, Chua C (2003) Solid freeform fabrication of three-dimensional scaffolds for engineering replacement tissues and organs. Biomaterials 24(13):2363-2378

14. Pilliar RM, Filiaggi MJ, Wells JD, Grynpas MD, Kandel RA (2001) Porous calcium polyphosphate scaffolds for bone substitute applications - in vitro characterization. Biomaterials 22(9):963972

15. Shanjani Y, Hu Y, Pilliar RM, Toyserkani E (2011) Mechanical characteristics of solid-freeform-fabricated porous calcium polyphosphate structures with oriented stacked layers. Acta Biomaterialia 7(4):1788-1796

16. Pilliar RM, Kandel RA, Grynpas MD, Zalzal P, Hurtig M (2007) Osteochondral defect repair using a novel tissue engineering approach: sheep model study. Technology and Health Care: Official Journal of the European Society for Engineering and Medicine 15(1):47-56

17. Grynpas MD, Pilliar RM, Kandel RA, Renlund R, Filiaggi M, Dumitriu M (2002) Porous calcium polyphosphate scaffolds for bone substitute applications in vivo studies. Biomaterials 23(9):2063-2070

18. Pilliar R, Hong J, Santerre P (2009) Method of manufacture of porous inorganic structures. US Patent 7494614

19. Shanjani Y, De Croos JNA, Pilliar RM, Kandel RA, Toyserkani E (2010) Solid freeform fabrication and characterization of porous calcium polyphosphate structures for tissue engineering purposes. Biomedical Materials Research Part B Applied Biomaterials 93(2):510-519

20. German R (1996) Sintering theory and practice, 1st edn. Wiley, New York

21. Gilman J, Vanderhart D, Kashiwagi T (1995) Thermaldecomposition chemistry of poly(vinyl alcohol) - characterization and reactions with bismaleimides. Fire and Polymers II: Materials and Tests for Hazard Prevention 599:161-185 


\title{
Solid freeform fabrication of porous calcium polyphosphate structures for bone substitute applications: In vivo studies
}

\author{
Yaser Shanjani, ${ }^{1}$ Youxin Hu, ${ }^{2}$ Ehsan Toyserkani, ${ }^{3}$ Marc Grynpas, ${ }^{4}$ Rita A. Kandel, ${ }^{4}$ Robert M. \\ Pilliar ${ }^{2}$ \\ ${ }^{1}$ Department of Orthopedic Surgery, Stanford University, Stanford, CA 94305 \\ ${ }^{2}$ Faculty of Dentistry, University of Toronto, 124 Edwards St., Toronto, Ontario, M5G 1 G6 Canada \\ ${ }^{3}$ Department of Mechanical and Mechatronics Engineering, University of Waterloo, Waterloo, Ontario, N2L 3G1 Canada \\ ${ }^{4} \mathrm{CIHR}$ - Bioengineering of Skeletal Tissues Team, Mount Sinai Hospital, Toronto, Ontario, M5G 1X5 Canada
}

Received 3 December 2012; revised 16 January 2013; accepted 17 January 2013

Published online 26 March 2013 in Wiley Online Library (wileyonlinelibrary.com). DOI: 10.1002/jbm.b.32905

\begin{abstract}
Porous calcium polyphosphate (CPP) structures with 30 volume percent porosity and made by solid freeform fabrication (SFF) were implanted in rabbit femoral condyle sites for 6-wk periods. Two forms of SFF implants with different stacked layer orientation were made in view of prior studies reporting on anisotropic/orthotropic mechanical properties of structures so formed. In addition, porous CPP implants of equal volume percent porosity made by conventional sintering and machining methods were prepared. Bone ingrowth and in vivo degradation of the three different implant types were compared using back-scattered scanning
\end{abstract}

electron microscopy (BS-SEM) of implant samples and quantitative analysis of the images. The results indicated bone ingrowth with all samples resulting in $30-40 \%$ fill of available porosity by bone within the 6 -wk period. In the 6 -wk in vivo period, approximately $7-9 \%$ loss of CPP by degradation had occurred. ( 2013 Wiley Periodicals, Inc. J Biomed Mater Res Part B: Appl Biomater 101B: 972-980, 2013.

Key Words: solid freeform fabrication, porous calcium polyphosphate, bone ingrowth, bone substitute, rabbit model study

How to cite this article: Shanjani Y, Hu Y, Toyserkani E, Grynpas M, Kandel RA, Pilliar RM. 2013. Solid freeform fabrication of porous calcium polyphosphate structures for bone substitute applications: In vivo studies. J Biomed Mater Res Part B 2013:101B:972-980.

\section{INTRODUCTION}

Autologous bone remains the preferred grafting material for repairing and replacing bone as a result of trauma, disease, or congenital deformity. ${ }^{1-3}$ It provides the four desired elements of an ideal bone substitute namely, osteoconductivity, osteoinductivity, ability to osseointegrate, and osteogenicity. However, autografting involves donor site morbidity, and at times, bone of required quantity or quality may not be available. To deal with such circumstances, extensive studies have been reported on alternative materials and structures for bone substitutes. ${ }^{4-15}$ These have included allografts and synthetic materials. Allografts have limitations related to potential disease transmission, immunogenicity, ready availability, and when making complex forms can be costly, a consequence of the thorough processing necessary to ensure their biocompatibility. Synthetic biomaterial-made bone substitutes, although less commonly used currently than allografts, offer potential advantages of ready availability, avoidance of incorporation of potential disease-transmitting organisms, and the ability to be custom-designed to satisfy form and local loading requirements. Like allografts, syn- thetics can be designed for osseointegration by forming structures suitable for implant anchorage in vivo by bone ingrowth or ongrowth. They can also be made to be osteoconductive through appropriate surface preparation (i.e., topographical and/or chemical composition design). ${ }^{16}$ Furthermore, synthetic bone substitute materials can be biodegradable, thereby allowing complete replacement in time by natural bone. ${ }^{17,18}$ An important requirement for bulk bone substitutes (as opposed to particulate forms) is the ability to resist fracture and excessive deformation under anticipated mechanical loading conditions both during the early healing process and for longer-term functional loading. Biodegradable synthetic bone substitute scaffolds made of organic polymers (PLA, PLLA-PGA copolymers) have been reported, but their limited mechanical properties make them unsuitable for some load-bearing applications. ${ }^{8,9}$ Previously, we have reported on the use of biodegradable porous inorganic structures made from calcium polyphosphate $\left(\mathrm{CPP} ;\left[\mathrm{Ca}\left(\mathrm{PO}_{3}\right)_{2}\right]_{\mathrm{n}}\right)$ as bone substitutes ${ }^{19-21}$ and preparation of so-called biphasic implants incorporating a bone substitute component to form novel osteochondral defect repair

Correspondence to: R. M. Pilliar; e-mail: bob.pilliar@utoronto.ca

Contract grant sponsors: Natural Sciences and Engineering Council of Canada and the U.S. Army Medical Research and Materiel Command \#W81XWH-10-1-0786 
implants. ${ }^{22-25}$ Bone ingrowth (and cartilage anchorage) into the porous structures was demonstrated in those previous studies. Porous CPP structures can be made by conventional powder sintering ${ }^{26,27}$ and machining of implants to desired form and porosity or by solid freeform fabrication (SFFalso referred to as additive manufacturing). The latter offers advantages in preparation of complex shapes and structures. Previous studies have also shown that higher compressive strength can be achieved for samples of a given percent porosity made by SFF compared with the conventional powder sintering and machining method. ${ }^{28}$ In addition, we showed that the mechanical properties of SFF-made CPP structures are dependent on the orientation of stacked layers. ${ }^{29}$

The current study was aimed at investigating the in vivo characteristics of structures formed by SFF by quantitative assessment of bone ingrowth and in vivo degradation of implants so formed. The study entailed implantation of porous CPP implants formed by SFF and direct comparison of bone ingrowth and degradation rates with conventionally sintered (CS) and machined implants placed in rabbit femoral condyle sites.

\section{MATERIALS AND METHODS}

\section{Materials and Fabrication Methods}

The preparation of CPP powder and methods for forming SFF and CS porous samples has been described elsewhere. ${ }^{19,26,28,29}$ In brief, CPP amorphous powders of 75$150 \mu \mathrm{m}$ mesh size were produced by calcining calcium phosphate monobasic monohydrate powders followed by melting and rapid quenching to form a glassy frit. This was then ground using an automated mortar and pestle to form amorphous CPP powders that were then screened to obtain powders of desired size. ${ }^{19}$ For preparation of samples by conventional methods (i.e., sintering and machining), the powders were packed into Pt tubes that served as molds and sintered to desired density using a two-step sinter/ anneal process. ${ }^{26}$ Cylindrical samples of desired dimensions were machined from sintered CPP rods.

For preparing the SFF-made samples, a mixture of amorphous CPP powder with a $10 \mathrm{wt} \%$ addition of polyvinyl alcohol (PVA) powder (to serve as a binder) was used for the layer-by-layer additive manufacturing process using a retrofitted 3D printer (ZPrinter 310 Plus - ZCorp) to create cylindrical samples as directed by a predetermined CAD model. An aqueous solvent $\left(\mathrm{Zb}^{\mathrm{TM}} 58\right.$ - ZCorporation, Burlington, MA) was injected to the successive layers during build-up causing dissolution of the PVA powder and formation of a binder that adhesively bonded the powders to allow formation of a "green" part. The SFF green samples so formed were then given post-SFF anneals to (i) burn-off the polymeric binder at a temperature below the glass transition temperature of the amorphous CPP; and (ii) sinter the CPP powder using the two-step annealing process. ${ }^{26}$ This two-step anneal results in rapid sinter neck formation between the amorphous powders as a result of viscous flow mechanism and development of a desired sample density at the lower step-1 annealing temperature ${ }^{27}$ followed by crystallization (devitrification) of the amorphous CPP during the heat-up and 1-h hold at the step-2 annealing treatment at $950^{\circ} \mathrm{C}$. Minimal additional sample densification occurs after crystallization with the $950^{\circ} \mathrm{C}, 1$-h anneal resulting in formation of $\beta$-CPP with crystals of approximately 1 - to $3-\mu \mathrm{m}$ size range. In this study, conditions were chosen to give CPP samples of approximately 30 volume percent porosity and interconnected pores in the 50 - to $150-\mu \mathrm{m}$ range (i.e., a pore size range suitable for rapid bone ingrowth).

Porous CPP samples (diameter $=4 \mathrm{~mm}$, height $=6$ $\mathrm{mm}$ ) produced by SFF directly to size and form and by CS by cutting from 4-mm diameter cylindrical rods using a diamond wafering blade were prepared for implantation in rabbit femoral condyle sites with implant positioning and orientation being similar to that used in previous studies. ${ }^{21}$ Previous mechanical property studies of SFF-made samples indicated that they displayed anisotropic (orthotropic) strength properties related to the powder build-up orientation during sample preparation (i.e., compressive strength = $33.86 \pm 6.32 \mathrm{MPa}$ when loaded normal to the lay-up direction [SSF-V samples] and $50.17 \pm 4.74 \mathrm{MPa}$ when loaded parallel to the lay-up direction [SSF-H samples]). To study if these orientation effects might affect bone ingrowth, SFFmade cylindrical rods were fabricated as reported previously $^{29}$ with powder layers deposited either perpendicular to the cylindrical sample axis to form SFF-V implants or parallel to the axis to form SFF-H implants (Figure 1).

\section{Porosity Characterization}

The porosity of each sintered sample was determined using the Archimedes method (ASTM C373) with ethanol as the buoyancy medium (Sartorius YDK01 Density determination Kit, Sartorius AG, Goettingen, Germany). The percent porosity of samples was determined by comparing the bulk density of the sintered samples and the theoretical density of nonporous CPP $\left(2.85 \mathrm{~g} / \mathrm{cm}^{3}\right) .{ }^{19,28}$ The percent porosities of one sample each of SFF-V and CS were also assessed after sectioning using quantitative image analysis of scanning electron microscopy (SEM) images taken of the prepared surfaces (see below). The comparison of percent porosity by this method confirmed that values determined by the
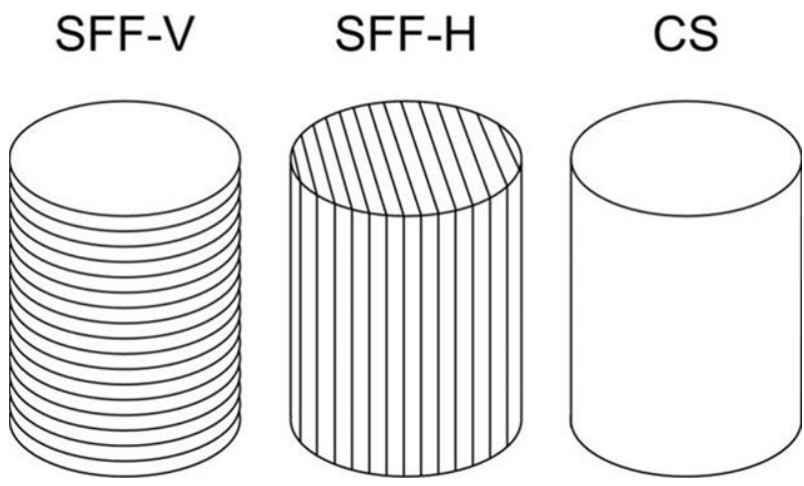

FIGURE 1. Schematic of SFF-V, SFF-H, and CS cylinders. SFF-made samples have been formed through a powder-based layer-by-layer manufacturing process. In SFF-V, the axis of cylinders was oriented perpendicular to the powder stacked-layers, whereas in SFF-H, the axis of cylinders was oriented parallel to the powder stacked layers. 


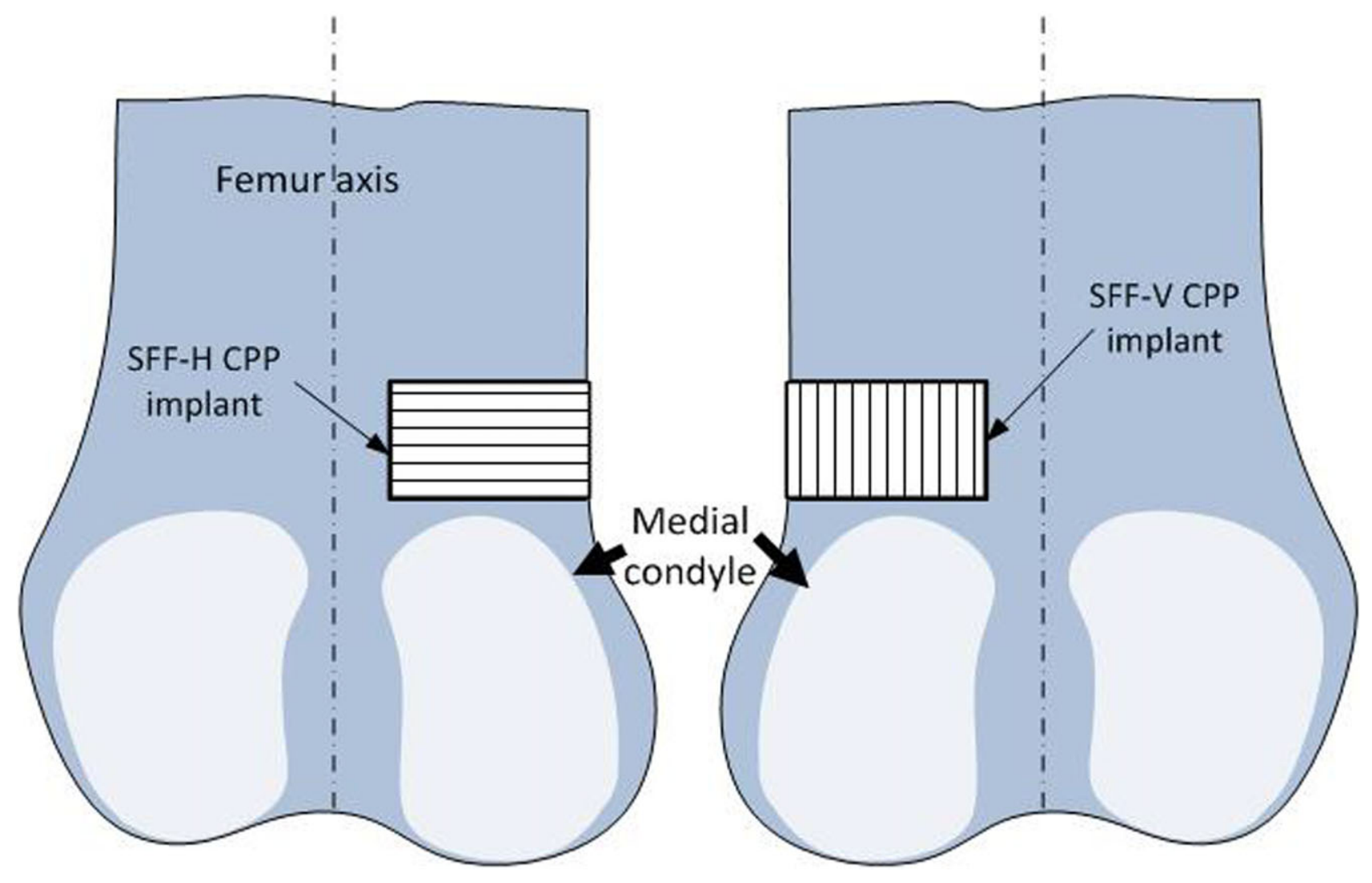

FIGURE 2. Schematic of CPP implants showing the layer orientation of SFF-made implants with respect to the implantation site. The layers are placed either parallel (in SFF-V) or perpendicular (in SFF-H) to the longitudinal bone axis (femur). [Color figure can be viewed in the online issue, which is available at wileyonlinelibrary.com.]

Archimedes and image analysis methods were the same (within acceptable experimental measuring error). This finding allowed use of the measured as-made densities of the samples in the in vivo implant degradation study as described below.

\section{Sample Implantation}

Rabbit implantations were conducted within the Faculty of Dentistry, University of Toronto with procedures approved by the University of Toronto Animal Care Committee.

The CPP implants were sterilized using gamma irradiation (2.5 Mrad) in preparation for implantation. A total of eight implants of each design (SFF-V, SFF-H, and CS) were placed transversely in the medial femoral condyles of both left and right femur of twelve 3.5- to 4-kg male New Zealand white rabbits (i.e., 24 implants in 12 animals with randomly selected dissimilar implant types in contralateral legs in each animal). Figure 2 shows how the SFF-V and SFF-H samples were positioned in the implantation sites. The SFF-made cylinders were oriented to align the constituent stacked-layers either parallel (SFF-V implants) or perpendicular (SFF-H implants) to the long axis of the rabbit femur (Figure 2) to determine if layer stacking orientation may have influenced bone ingrowth. All rabbits were ambulatory $24 \mathrm{~h}$ postsurgery and were sacrificed after 6 wks.

\section{In vivo Assessment}

Implant-bone blocks, harvested from the rabbits, were fixed in $10 \%$ buffered formalin solution for approximately $1 \mathrm{wk}$. Dehydration was achieved using ethyl alcohol (70\% for 6 days followed by $100 \%$ for an additional 6 days). The samples were then left in xylene overnight and then infiltrated with Osteo-Bed resin using standard resin embedding methods as specified by the supplier (Polysciences, Warrington, PA). After curing of the resin, one implant-bone block sample of each type (i.e., SFF-H, SFF-V, and CS) was sectioned transversely to its long axis using a diamond wafering blade (Buehler Isomet Low Speed Saw - Lake Bluff, IL) to prepare a transverse section for qualitative assessment of bone ingrowth. The remaining seven samples of each type were sectioned longitudinally for subsequent quantitative image analysis. One of each was examined before further sectioning (see below for method for preparing sections for quantitative image analysis) to obtain a qualitative assessment of extent of bone ingrowth along the implant length. Preparation of the samples for quantitative assessment of bone ingrowth and degradation after the 6-wk implantation period involved further sectioning of the samples along the implant axis and in the anterior-posterior and proximal-distal directions to provide four segments per implant for examination (Figure 3). The cut surfaces of all samples for both qualitative and quantitative assessment were polished with a series of graded silicon carbide papers (final grade equal to 4000 grit) and the 


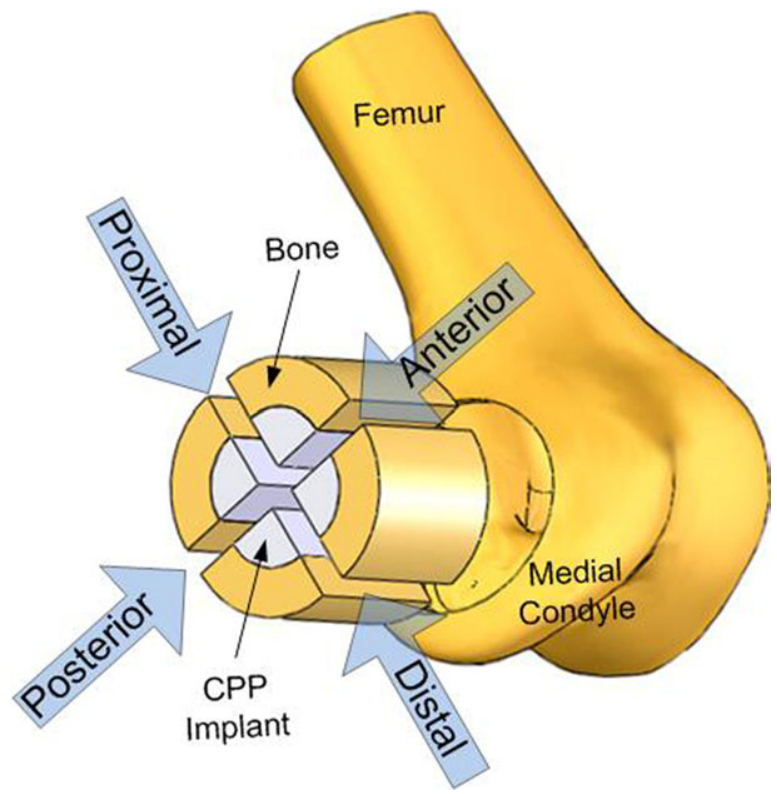

FIGURE 3. Schematic of sectioning of inserted CPP implants. Each implant was sectioned longitudinally and transversely relative to femur long axis and through the center of the implant, generating four implant surfaces (anterior, posterior, distal, and proximal) for quantitative analysis of bone ingrowth. [Color figure can be viewed in the online issue, which is available at wileyonlinelibrary.com.]

polished surfaces examined by back-scattered SEM (BS-SEM). Captured back-scattered electron (BSE) images were used for the quantitative image analysis as described below. After BSE imaging, thin sections of some samples were further prepared for a histologic examination.

For the quantitative image analysis of bone ingrowth and CPP degradation, six samples of SFF-V, seven samples of SFF$\mathrm{H}$, and seven samples of CS were examined. (One of the SFF-V samples was lost through a technical error during the section processing). The SFF-V, SFF-H, and CS CPP groups were compared, by analysis of the cut and polished sections from the four different aspects (anterior, posterior, distal, and proximal) of the retrieved implant-bone samples (Figure 3). For quantitative analysis of bone ingrowth and CPP degradation, the cut and polished implant-bone section surfaces were coated with a thin $(8 \mathrm{~nm})$ platinum film in preparation for SEM examination. A Hitachi S-2500 SEM (Hitachi Ltd, Tokyo, Japan) operated at an accelerating voltage of $25 \mathrm{kV}$ was used for the examination. BSE images were captured and used for quantitative analysis of percentage bone ingrowth and CPP degradation after 6 wks in vivo. For this purpose, using PRTools (a MATLAB-based toolbox for pattern recognition [http://www.prtools.org]), the BSE images were segmented by contrast into three discrete segments: (1) bone, (2) CPP, and (3) nonmineralized tissues. The constituent percentage of each section was calculated by a MATLAB ${ }^{\mathrm{TM}}$ code.

The percentage of the pores filled by newly formed bone was calculated as:

$$
\% \text { Bone }- \text { in }- \text { Pores }=\frac{\text { Bone area }}{(\text { Bone }+ \text { Pore }) \text { area }} \times 100
$$

For the qualitative histologic assessment (focussed on the interface zone region), three representative implants, one per each CPP implant type, were used for preparing sections for examination by light microscopy. For this purpose, the polished surfaces prepared for BS-SEM examination were stained using $0.3 \%$ toluidine blue and $2 \%$ sodium borate in a $1: 1$ solution for $15 \mathrm{~min}$ at $50^{\circ} \mathrm{C}$. After rinsing with 70 and $100 \%$ ethanol, the blocks were air dried. The blocks were then counterstained with $0.2 \%$ light green in $0.2 \%$ acetic acid for 1-3 min at room temperature followed by rinsing in $100 \%$ ethanol and air dried. The stained block face of each segment was then glued to a microscope slide with epoxy and left overnight. Sections were cut with a 3-inch diameter diamond wafering blade to a thickness of approximately $150 \mu \mathrm{m}$. The sections were then polished to approximately $20 \mu \mathrm{m}$ thicknesses using a series of silicon carbide papers finishing with a 4000 grit paper. The stained sections were examined by light microscopy.

\section{Degradation Analysis}

Estimates of CPP degradation were made by comparing final percent of CPP (determined by analysis of BSE images) to the starting volume percent density (determined by the Archimedes method and confirmed to be the same as determined by image analysis of controlled samples before implantation).

\section{Statistical Analysis}

One-way analysis of variance was conducted to compare volume percent porosity of the SFF- and CS-prepared implants and percent bone ingrowth/available porosity for the different implant types with significant differences being assigned for $F$ values greater than an $F_{\text {critical }}$ corresponding to $P<0.05$.

\section{RESULTS}

\section{Porosity and Pore Size Distribution}

The results of the volume percent porosity determinations for all CPP samples in the as-made condition (i.e., before implantation) are listed in Table I (in average $\sim 30$ volume percent). There was no significant difference in percent porosity between the sample types. In addition, on the basis of our previous findings of samples of slightly greater percent porosity ( $\sim 35$ volume percent porosity), in which the

TABLE I. Percent Full Theoretical Density of CPP Implants Measured by Archimedes Method

\begin{tabular}{lccc}
\hline Sample \# & SFF-V & SFF-H & CS \\
\hline 1 & 74.05 & 73.70 & 70.71 \\
2 & 71.77 & 73.79 & 72.40 \\
3 & 73.03 & 70.22 & 69.46 \\
4 & 72.73 & 66.87 & 70.33 \\
5 & 72.65 & 70.41 & 69.91 \\
6 & 70.35 & 66.14 & 68.87 \\
7 & 70.06 & 69.89 & 68.35 \\
8 & 72.21 & 66.54 & 70.37 \\
Average & $\mathbf{7 2 . 1 1}$ & $\mathbf{6 9 . 6 9}$ & $\mathbf{7 0 . 0 5}$ \\
SD & $\mathbf{1 . 3 5}$ & $\mathbf{3 . 0 3}$ & $\mathbf{1 . 2 4}$ \\
\hline
\end{tabular}



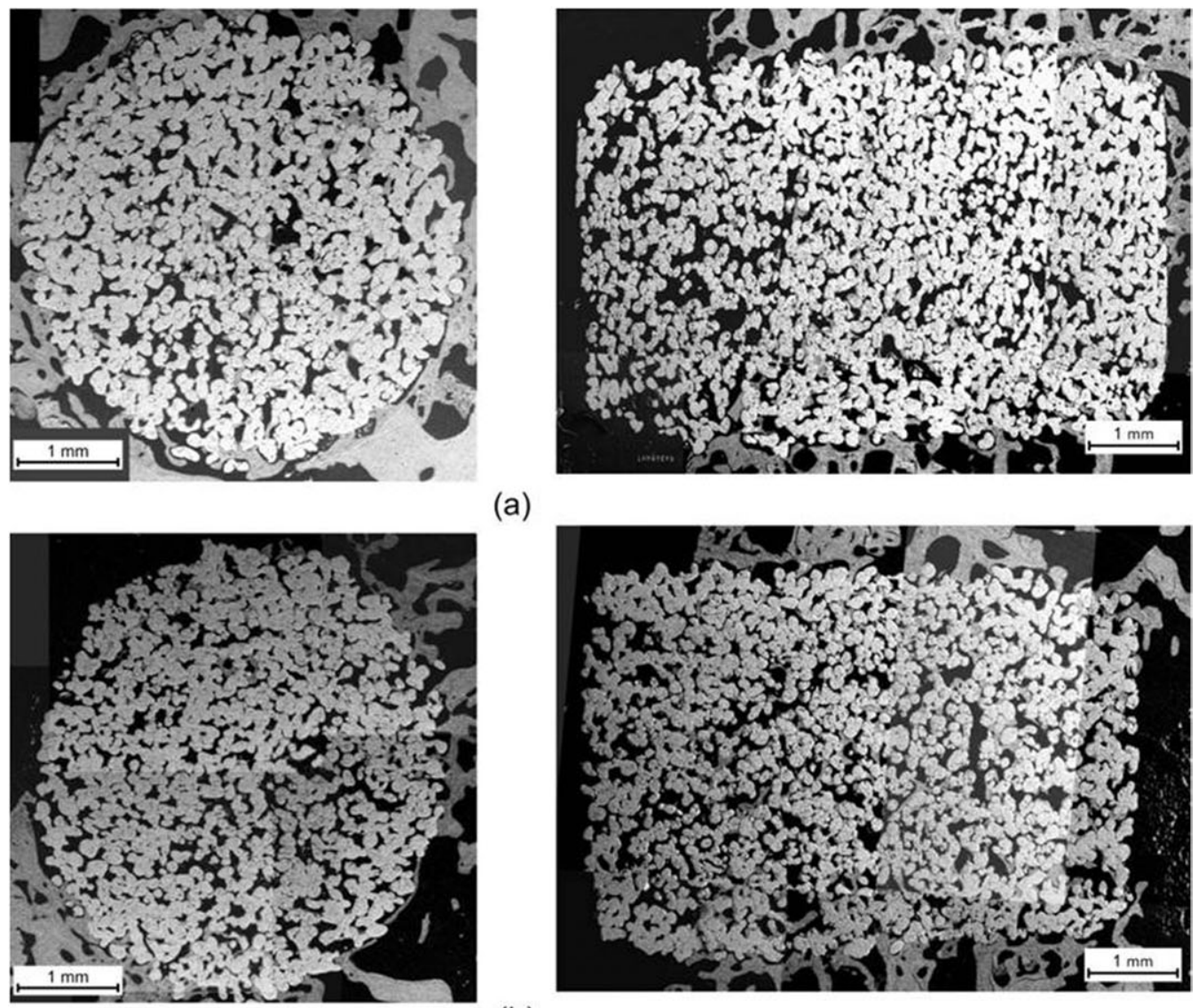

(a)

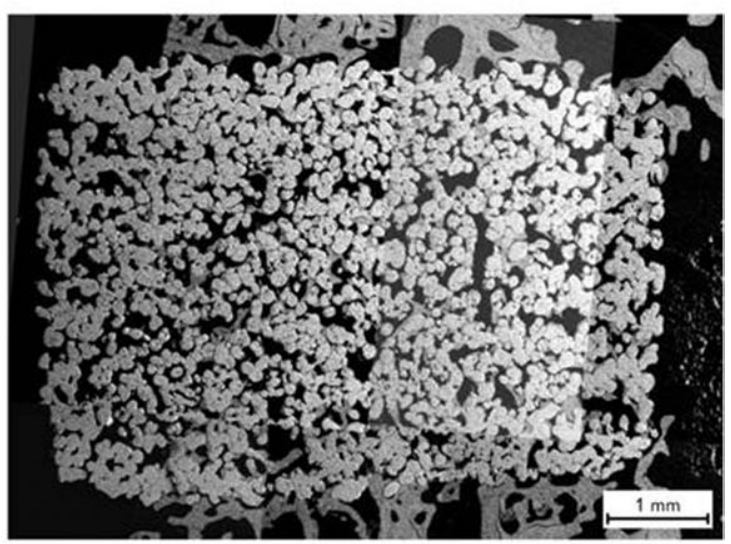

(b)
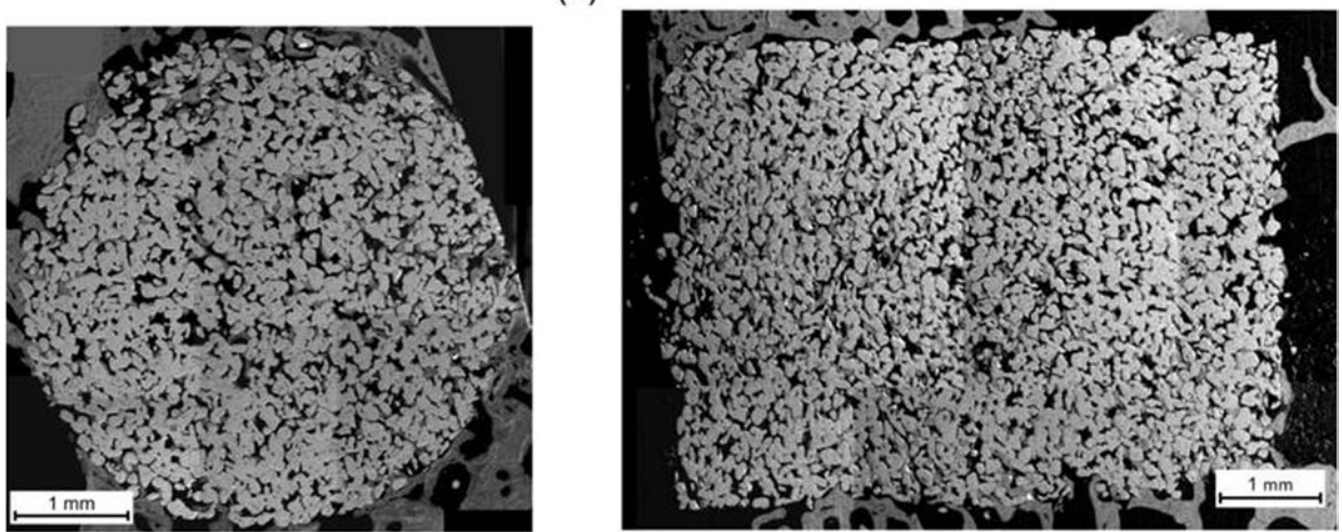

(c)

FIGURE 4. BSE images of radial and longitudinal cross-sections: (a) SFF-V, (b) SFF-H, and (c) CS samples. New bone was seen throughout the 4-mm cross-sectional diameter of the porous CPP implants and the implants appeared securely fixed by bone ingrowth.

pore size and pore size distribution were determined using high-resolution microcomputed tomography, ${ }^{29}$ it is expected that the mean pore size for the CS samples is smaller. (The previous micro-CT assessment of $65 \%$ dense samples indicated mean pore size $\approx 56 \mu \mathrm{m}$ for SFF-made samples with a pore size range of 5-140 $\mu \mathrm{m}$ compared with a mean pore size $\approx 38 \mu \mathrm{m}$ and range of $5-80 \mu \mathrm{m}$ for CS samples ${ }^{29}$ ). Similar results with only minor variation are expected for the $70 \%$ dense (30 volume percent porous) CPP structures used in the current study.
In vivo Response-Bone Ingrowth

As previously reported ${ }^{20,21}$ and as confirmed by the histology assessment in this study, the porous CPP structures appeared to be biocompatible (i.e., no cytotoxic or chronic inflammatory response after the 6-wk implantation period).

Qualitative assessments of the BSE images from the sectioned samples (transverse and longitudinal to the implant axis) are shown in Figures $4(\mathrm{a}-\mathrm{c})$. New bone was seen throughout the 4-mm cross-sectional diameter of the porous CPP implants, and the implants appeared securely fixed by 
Host bone-CPP interface

(a)

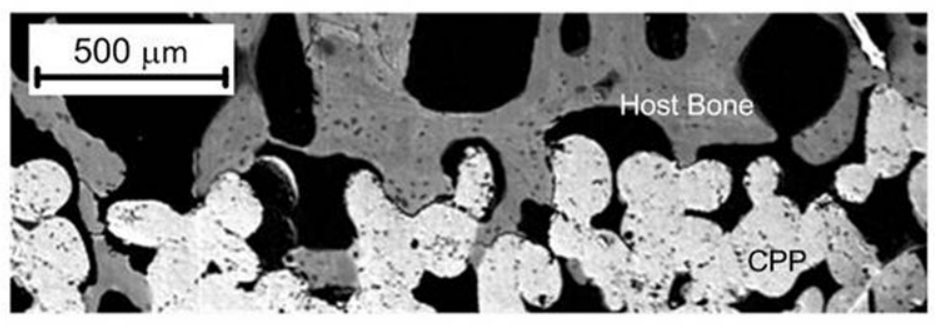

(b)

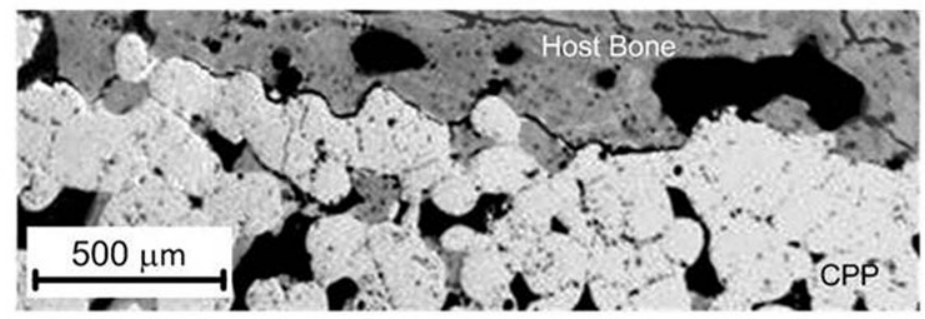

(c)

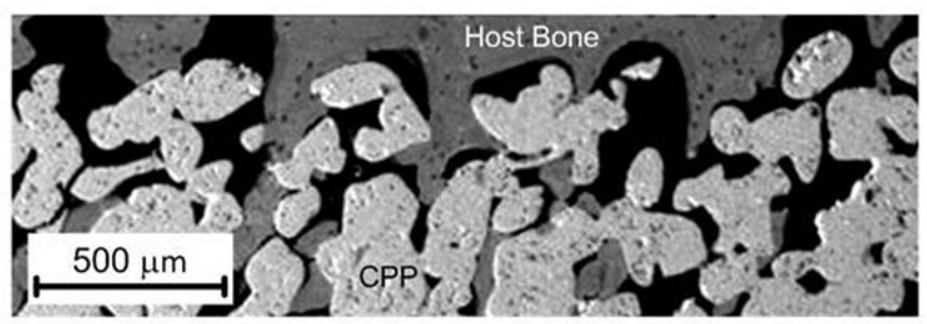

Core region
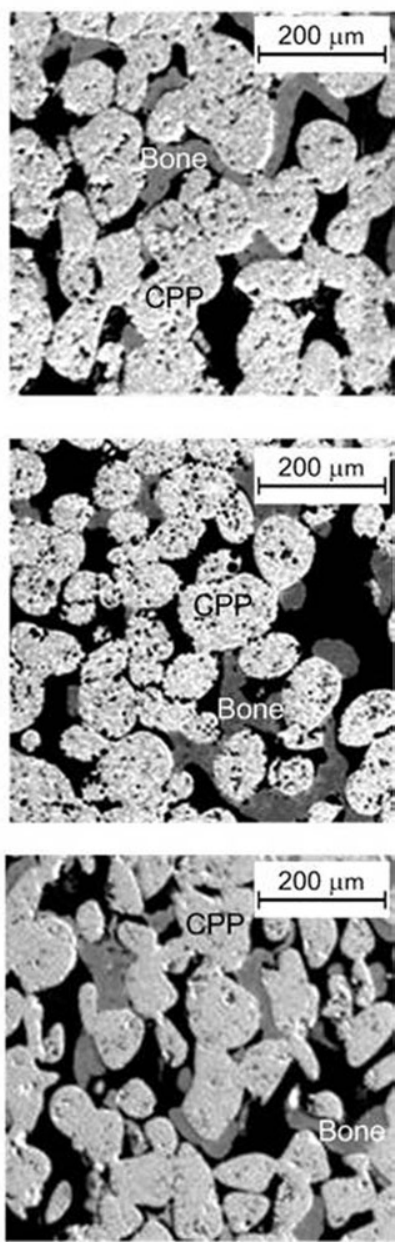

FIGURE 5. BSE images showing (right) bone formation in core region of implants and (left) the integration of CPP constructs and host bone in the interface of (a) SFF-V, (b) SFF-H, and (c) CS implants and surrounding bone. Qualitatively significant bone ingrowth within the different regions of the implants, including the central core regions and toward the circumferential regions nearest the host bone junction was observed. Bone ingrowth appeared uniformly distributed throughout the implants.

bone ingrowth. The BSE images revealed qualitatively significant bone ingrowth within the different regions of the implants, including the central core regions and toward the circumferential regions nearest the host bone junction (Figure 5). In addition, the bone ingrowth appeared uniformly distributed throughout the implants.

The percentages of available pores filled by bone for the SFF-V, SFF-H, and CS implants as determined by the quantitative image analysis are shown in Table II. The average values indicate that new bone had filled approximately $33-36 \%$ of the available pore area within the CPP implants by 6 wks. There was no significant difference in percent bone ingrowth/available pore area for the four different implant segments (anterior, posterior, proximal, and distal) for any of the implants. In addition, there was no significant difference in percent bone ingrowth/ available pore area between the SFF- and CS-made samples.

TABLE II. Percentage of Void Area Filled With Bone (\%Bone-in-Pores) of SFF-V, SFF-H, and CS CPP Implants in the Anterior, Posterior, Distal, and Proximal Aspects

\begin{tabular}{llllll}
\hline & Anterior & Posterior & Distal & Proximal & Ave \pm SEM \\
\hline SFF-V & $39.01 \pm 2.56$ & $33.61 \pm 4.44$ & $36.00 \pm 3.48$ & $31.44 \pm 1.27$ & $35.01 \pm 3.25$ \\
SFF-H & $35.41 \pm 3.43$ & $33.58 \pm 4.04$ & $31.94 \pm 7.67$ & $32.65 \pm 7.22$ & $33.39 \pm 1.50$ \\
CS & $35.99 \pm 5.91$ & $37.46 \pm 13.92$ & $32.70 \pm 10.32$ & $39.38 \pm 4.78$ & $36.38 \pm 2.82$ \\
\hline
\end{tabular}

The average values indicate that new bone had filled approximately $30-40 \%$ of the available pore area within the CPP implants by 6 wks with no significant for SFF-made and CS implants and for the four different implant segments (anterior, posterior, proximal, and distal) for any of the implants. 
TABLE III. Percentage of Degradation of SFF-V, SFF-H, and CS CPP Implants in the Anterior, Posterior, Distal, and Proximal Aspects

\begin{tabular}{lrrrrr}
\hline & \multicolumn{1}{c}{ Anterior } & \multicolumn{1}{c}{ Posterior } & \multicolumn{1}{c}{ Distal } & Proximal & Ave \pm SEM \\
\hline SFF-V & $11.72 \pm 5.42$ & $10.50 \pm 2.12$ & $8.49 \pm 6.23$ & $5.59 \pm 2.02$ & $9.08 \pm 2.67$ \\
SFF-H & $9.09 \pm 8.85$ & $6.92 \pm 4.47$ & $10.59 \pm 4.35$ & $5.18 \pm 6.30$ & $7.94 \pm 2.38$ \\
CS & $9.55 \pm 2.04$ & $5.81 \pm 1.72$ & $5.60 \pm 0.71$ & $8.95 \pm 2.05$ & $7.48 \pm 2.06$
\end{tabular}

The data confirm that degradation does occur and provide an indication of the extent of CPP loss in the 6-wk in vivo period.

\section{CPP Degradation}

After the 6-wk implantation period, a reduction in overall amount of CPP was noted as determined by percent area of $\mathrm{CPP} /$ implant and, hence, volume percent CPP/implant (Table III). The extent of degradation was determined through quantitative image analysis of CPP regions after implant retrieval, estimation of average CPP particle percent area, and comparison to the known starting percent CPP. CPP loss for the implants ranged from approximately 7-9\%. The observed large standard deviations noted for the quantitative image assessment of percent CPP retained in the implanted samples in the different aspects are due to the limited number of sections assessed in this study, which involved examining only the mid-plane/axial anterior-posterior and proximal-distal cut surfaces in each implant. More extensive evaluation using additional cut sections would be required for a more precise estimate by quantitative image analysis of percent CPP loss. The current analysis, however, does confirm that degradation does occur and provides an indication of the extent of CPP loss in the 6-wk in vivo period.

\section{Histologic Analysis}

Histologic examination of thin sections prepared from the retrieved bone-implant blocks confirmed the formation of extensive bone ingrowth for the three sample types by the 6-wk period. There was no indication of an adverse tissue reaction confirming the biocompatible nature of the porous
CPP samples made either by SFF or conventional sintering and machining methods. Figure 6 shows a micrograph of a representative stained section of the bone-implant interface region (SFF-H sample).

\section{DISCUSSION}

Preparation of porous CPP samples by the SFF method offers the potential advantage of complete or near-net final form fabrication of implants without the need for extensive machining. In addition, for clinical applications, it is possible to produce custom-made patient-specific implants by combining medical imaging, 3D CAD modeling and the SFF method. ${ }^{30,31}$ This process creates more accurate implants that, in turn, require less invasive surgeries for implant insertion. It also offers the possibility of incorporating internal features within complex forms.

Two features that have been identified in previous publications distinguish components made by SFF compared with CS fabrication. As observed qualitatively in the current study and as reported quantitatively previously, ${ }^{28}$ despite the use of similar starting powder size, SFF-made samples result in larger average pore size. A possible cause of this difference as discussed in a previous article ${ }^{29}$ is related to the different Step-1 sinter temperatures used for SFF- and CS-made structures. The delayed sinter neck development by viscous flow of amorphous CPP and consequent need to use a higher Step- 1 sinter anneal temperature to make porous samples of $70 \%$ density (i.e., 30 volume percent

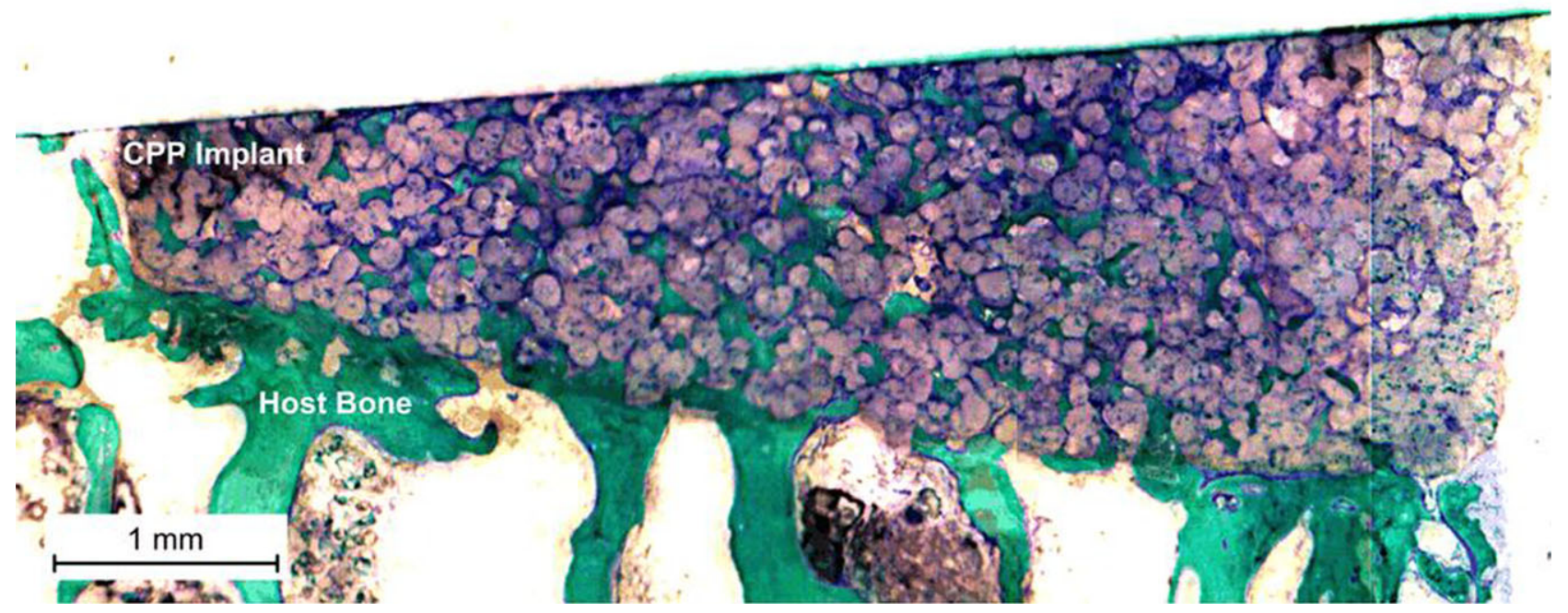

FIGURE 6. Histology image of an SFF-H implant (as a representative of SFF-made samples): green color represents for the mineralized bone tissue. Significant bone ingrowth is observed filling the pores of implant. [Color figure can be viewed in the online issue, which is available at wileyonlinelibrary.com.] 
porosity) has been proposed as the reason for this. The higher Step-1 anneal is expected to result in greater mass transport and pore coarsening (an Ostwald ripening type of phenomenon ${ }^{32}$ ). A second difference for the SFF- and CS-made samples is the introduction of a polymer binder phase with the former. The possible consequence of this has also been noted in a previous publication ${ }^{29}$ and raises the question as to whether this might in some way alter the in vivo response of porous CPP implants and affect bone ingrowth characteristics. Hence, the current study was undertaken. The major findings of this study indicate that porous CPP implants formed by the SFF method seem to be the same in terms of bone ingrowth characteristics to CS and machined samples. The result is not surprising in view of the generally accepted finding from many previous studies over the past number of decades that uninhibited bone ingrowth will occur into porous structures provided that the implant is made of biocompatible material, the pore size is greater or equal to approximately $100 \mu \mathrm{m}$ and that adequate vascularity, and good osteogenic cell populations and conditions for bone formation and ingrowth (limited initial relative movement at the implant-bone junction and aseptic conditions) are satisfied. ${ }^{33,34}$ The osteoconductive nature of calcium phosphates in general, including porous CPP, further supports rapid bone ingrowth leading to the successful implant osseointegration of the SFF-made and CS implants observed in the current study.

A further question that was prompted by an earlier observation of anisotropic/orthotropic mechanical strengths of SFF-made samples was whether this may have had an effect on the rate of bone ingrowth. As discussed elsewhere, the anisotropic effect was attributed to differences in sinter neck geometry for SFF-made samples in the direction parallel and perpendicular to constituent stacked layers. ${ }^{29}$ This was related to use of CPP powders with aspect ratios $>1$ and the consequent effect of this on powder orientation during layer-by-layer build up. This, in turn, affected sinter neck geometry and introduced an anisotropic effect that was significant with respect to compressive strength and, as noted in our further studies (unreported data), bending strength and implant stiffness as well. However, the current study did not demonstrate any anisotropic effect on bone ingrowth characteristics. It is recognized that greater structural anisotropy could be incorporated into implants using the SFF method, but it remains to be shown if this could have a clinically significant effect in terms of implant osseointegration or preferred functional performance of porous CPP implants. In view of the biodegradation nature of CPP, this does not seem likely. Over the long term, functional loading and bone remodeling in harmony with CPP degradation are likely to determine performance. However, on the basis of observed anisotropic properties, the stackedlayer orientation can be selected during SFF formation of implant components such that optimal load bearing characteristic can be designed into the structures, that is, highest strength direction being chosen to match with an anticipated principal load/stress direction during functional loading.
Finally, a comment concerning the CPP degradation rates reported in this study. As already noted, the estimates of CPP loss during the 6-wk implantation period determined by image analysis of a limited number of polished sections should be treated as preliminary. More extensive studies involving serial sectioning of samples and their analysis should be undertaken to provide a more reliable estimate of in vivo CPP degradation. In addition, with degradation rates as the primary focus, additional implantation time periods should be studied. Comparisons with previously reported in vivo degradation of porous CPP samples are unreliable because CPP degradation rates are strongly dependent on the process used to form both CPP starting powders, powder size, and sintering schedules used to form porous structures parameters, which, during the course of our research, have been modified from our early reported studies. A controlled degradation rate of CPP implants allows for gradual load transfer to bone, increasing space for bone ingrowth, and eventual filling of a defect with natural bone. ${ }^{35}$ Longer degradation rates may be necessary for larger animals or larger defect sites because of a slower repair process, which should be investigated further in future studies. ${ }^{36}$ Mineralization of the new bone tissue may have been accelerated by the presence of ions resulting from the degradation of the CPP, that is, $\mathrm{Ca}^{2+}$ and $\left(\mathrm{PO}_{4}\right)^{3-20}$ The degradation products do not seem to incite any inflammatory reaction. ${ }^{22}$

The current studies are encouraging, however, because they suggest that for porous CPP implants made as described in this study (either by SFF or conventional sintering and machining methods), degradation rates are in the range that would make porous CPP implants suitable for bone substitute/augment applications. Furthermore, the strengths previously reported for porous CPP structures made by SFF and suitable for rapid bone ingrowth suggest their potential as bone substitutes in load-bearing sites.

This study has shown that porous CPP implants made by SFF technique to form structures with 30 volume percent porosity and with pores in the 50 - to $150-\mu \mathrm{m}$ size range placed in rabbit femoral condyle sites for 6-wk periods behave similarly with respect to bone ingrowth and in vivo degradation to implants of similar volume percent porosity made by conventional powder sintering and machining methods. Both methods resulted in formation of acceptable biocompatible implants. This suggests the potential of SFF for making complex shapes suitable for bone substitute or bone augment applications, even in load-bearing skeletal regions. Further studies to more completely characterize the degradation rates and response to long-term implantation to study the eventual replacement of the CPP elements by bone and concomitant bone remodeling are recommended.

\section{ACKNOWLEDGMENTS}

The authors thank Ms. Nancy Valiquette for her invaluable assistance with the histology and BS-SEM sample preparation.

\section{REFERENCES}

1. Zimmermann G, Moghaddam A. Allograft bone matrix versus synthetic bone graft substitutes. Injury 2011;42:S16-S21. 
2. Walsh WR, Vizesi F, Michael D, Auld J, Langdown A, Oliver R, Yu $Y$, Irie $H$, Bruce W. $\beta$-TCP bone graft substitutes in a bilateral rabbit tibial defect model. Biomaterials 2008;29:266-271.

3. Wagoner Johnson AJ, Herschler BA. A review of the mechanical behavior of $\mathrm{CaP}$ and $\mathrm{CaP} /$ polymer composites for applications in bone replacement and repair. Acta Biomater 2011;7:16-30.

4. Dobbs HS, Robertson JLM. Heat treatment of cast Co-Cr-Mo for orthopaedic implant use. J Mater Sci 1983:18:391-401.

5. Beddoes J, Bucci K. The influence of surface condition on the localized corrosion of $316 \mathrm{~L}$ stainless steel orthopaedic implants. J Mater Sci Mater Med 1999;10:389-394.

6. Long M, Rack HJ. Titanium alloys in total joint replacement-A materials science perspective. Biomaterials 1998;19:1621-1639.

7. Bobyn JD, Stackpool GJ, Hacking SA, Tanzer M, Krygier JJ. Characteristics of bone ingrowth and interface mechanics of a new porous tantalum biomaterial. J Bone Joint Surg Br 1999;81:907-914.

8. Athanasiou KA, Agrawal CM, Barber FA, Burkhart SS. Orthopaedic applications for PLA-PGA biodegradable polymers. Arthroscopy 1998;14:726-737.

9. Thomson RC, Yaszemski MJ, Powers JM, Mikos AG. Fabrication of biodegradable polymer scaffolds to engineer trabecular bone. J Biomater Sci Polym Ed 1995;7:23-38.

10. Shimko DA, Nauman EA. Development and characterization of a porous poly(methyl methacrylate) scaffold with controllable modulus and permeability. J Biomed Mater Res B Appl Biomater 2007;80:360-369.

11. Spector M, Michno MJ, Smarook WH, Kwiatkowski GT. A highmodulus polymer for porous orthopedic implants: Biomechanical compatibility of porous implants. J Biomed Mater Res 1978;12: 665-677.

12. Choueka J, Charvet JL, Koval KJ, Alexander H, James KS, Hooper KA, Kohn J. Canine bone response to tyrosine-derived polycarbonates and poly(L-lactic acid). J Biomed Mater Res 1996;31: 35-41.

13. Williams JM, Adewunmi A, Schek RM, Flanagan $C L$, Krebsbach $\mathrm{PH}$, Feinberg SE, Hollister SJ, et al. Bone tissue engineering using polycaprolactone scaffolds fabricated via selective laser sintering. Biomaterials 2005;26:4817-4827.

14. Eggli PS, Müller W, Schenk RK. Porous hydroxyapatite and tricalcium phosphate cylinders with two different pore size ranges implanted in the cancellous bone of rabbits. A comparative histomorphometric and histologic study of bony ingrowth and implant substitution. Clin Orthop Relat Res 1988;232:127-138.

15. Chu TMG, Orton DG, Hollister SJ, Feinberg SE, Halloran JW. Mechanical and in vivo performance of hydroxyapatite implants with controlled architectures. Biomaterials 2002;23:1283-1293.

16. Bohner M. Resorbable biomaterials as bone graft substitutes. Mater Today 2010;13:24-30.

17. Ryu W, Hammerick KE, Kim YB, Kim JB, Fasching R, Prinz FB. Three-dimensional biodegradable microscaffolding: Scaffold characterization and cell population at single cell resolution. Acta Biomater 2011;7:3325-3335.

18. LeGeros RZ. Biodegradation and bioresorption of calcium phosphate ceramics. Clin Mater 1993;14:65-88.
19. Pilliar RM, Filiaggi MJ, Wells JD, Grynpas MD, Kandel RA. Porous calcium polyphosphate scaffolds for bone substitution applications-In vitro characterization. Biomaterials 2001;22:963-972.

20. Pilliar RM, Filiaggi MJ, Wells JD, Grynpas MD, Kandel RA. Porous calcium polyphosphate scaffolds for bone substitution applications-In vitro characterization. Biomaterials 2001;22:963-972.

21. Pilliar RM, Grynpas MD, Kandel RA, Hu Y. Porous calcium polyphosphate as load-bearing bone substitutes: In vivo study. J Biomed Mater Res B Appl Biomater 2013;101:1-8.

22. Kandel RA, Grynpas M, Pilliar R, Lee J, Wang J, Waldman S, Zalzal $P$, et al. Repair of osteochondral defects with biphasic cartilage-calcium polyphosphate constructs in a sheep model. Biomaterials 2006;27:4120-4131.

23. Kandel RA, Pilliar R, Grynpas M. Biphasic constructs for cartilage repair. Eur Cells Mater 2007;13:10.

24. Allan KS, Pilliar RM, Wang J, Grynpas MD, Kandel RA. Formation of biphasic constructs containing cartilage with a calcified zone interface. Tissue Eng 2007;13:167-177.

25. Waldman SD, Grynpas MD, Pilliar RM, Kandel RA. Characterization of cartilaginous tissue formed on calcium polyphosphate substrates in vitro. J Biomed Mater Res 2002;62:323-330.

26. Pilliar RM, Hong J, Santerre PJ. Method of manufacture of porous inorganic structures. US patent 7,494,614; 2009.

27. Filiaggi M, Pilliar RM, Hong J. On the sintering characteristics of calcium polyphosphates. Key Eng Mater 2001;192-195:171-174.

28. Shanjani Y, De Croos JNA, Pilliar RM, Kandel RA, Toyserkani E. Solid freeform fabrication and characterization of porous calcium polyphosphate structures for tissue engineering purposes. J Biomed Mater Res Part B: Appl Biomater 2010;93B:510-519.

29. Shanjani Y, Hu Y, Pilliar RM, Toyserkani E. Mechanical characteristics of solid-freeform-fabricated porous calcium polyphosphate structures with oriented stacked layers. Acta Biomater 2011;7: 1788-1796.

30. Poukens J, Laeven P, Beerens M, Nijenhuis G, Sloten JV, Stoelinga $\mathrm{P}$, Kessler P. A classification of cranial implants based on the degree of difficulty in computer design and manufacture. Int $\mathrm{J}$ Med Robot Comput Assist Surg 2008;4:46-50.

31. Salmi M, Tuomi J, Paloheimo K, Björkstrand R, Paloheimo $M$, Salo J, Kontio $R$, et al. Patient-specific reconstruction with 3D modeling and DMLS additive manufacturing. Rapid Prototyping $\mathrm{J}$ 2012;18:209-214.

32. Rahaman MN. Ceramic Processing and Sintering. USA: Routledge; 2003. pp 875-412.

33. Nguyen HQ, Deporter DA, Pilliar RM, Valiquette N, Yakubovich R. The effect of sol-gel-formed calcium phosphate coatings on bone ingrowth and osteoconductivity of porous-surfaced $\mathrm{Ti}$ alloy implants. Biomaterials 2004;25:865-876.

34. Pilliar RM. Porous-surfaced metallic implants for orthopedic applications. J Biomed Mater Res 1987;21:1-33.

35. Mistry AS, Mikos AG. Tissue engineering strategies for bone regeneration. Adv Biochem Eng Biotechnol 2005;94:1-22.

36. Keeney M, Pandit A. The osteochondral junction and its repair via bi-phasic tissue engineering scaffolds. Tissue Eng Part B 2009;15: 55-73. 


\title{
Porous calcium polyphosphate bone substitutes: Additive manufacturing versus conventional gravity sinter processing-Effect on structure and mechanical properties
}

\author{
Youxin Hu, ${ }^{1}$ Yaser Shanjani, ${ }^{2}$ Ehsan Toyserkani, ${ }^{2}$ Marc Grynpas, ${ }^{1,3}$ Rizhi Wang, ${ }^{4}$ Robert Pilliar ${ }^{1,5 *}$ \\ ${ }^{1}$ Institute of Biomaterials and Biomedical Engineering, University of Toronto, Toronto, Ontario, Canada M5S 3G9 \\ ${ }^{2}$ Department of Mechanical and Mechatronics Engineering, Multi-Scale Additive Manufacturing Laboratory, University of \\ Waterloo, Waterloo, Ontario, Canada N2L 3G1 \\ ${ }^{3}$ CIHR-Bioengineering of Skeletal Tissues Team, Mount Sinai Hospital, Toronto, Ontario, Canada M5G 1X5 \\ ${ }^{4}$ Department of Materials Engineering, University of British Columbia, Vancouver, British Columbia, Canada V6T 1 Z4 \\ ${ }^{5}$ Faculty of Dentistry, University of Toronto, Toronto, Ontario, M5G 1G6, Canada
}

Received 15 March 2013; revised 2 June 2013; accepted 1 July 2013

Published online 00 Month 2013 in Wiley Online Library (wileyonlinelibrary.com). DOI: 10.1002/jbm.b.33005

\begin{abstract}
Porous calcium polyphosphate (CPP) structures proposed as bone-substitute implants and made by sintering CPP powders to form bending test samples of approximately $35 \mathrm{vol} \%$ porosity were machined from preformed blocks made either by additive manufacturing (AM) or conventional gravity sintering (CS) methods and the structure and mechanical characteristics of samples so made were compared. AM-made samples displayed higher bending strengths $(\approx 1.2-1.4$ times greater than CS-made samples), whereas elastic constant (i.e., effective elastic modulus of the porous structures) that is determined by material elastic modulus and structural geometry of the samples was $\approx 1.9-2.3$ times greater for AM-made samples. X-ray diffraction analysis showed that samples made by either method displayed the same crystal structure forming $\beta$-CPP after sinter annealing. The material elastic modulus, $E$, determined using nanoindentation tests also showed the same value for both sample types (i.e., $E \approx 64 \mathrm{GPa}$ ). Examination of the porous structures indicated that significantly larger sinter necks resulted in the
\end{abstract}

AM-made samples which presumably resulted in the higher mechanical properties. The development of mechanical properties was attributed to the different sinter anneal procedures required to make $35 \mathrm{vol} \%$ porous samples by the two methods. A primary objective of the present study, in addition to reporting on bending strength and sample stiffness (elastic constant) characteristics, was to determine why the two processes resulted in the observed mechanical property differences for samples of equivalent volume percentage of porosity. An understanding of the fundamental reason(s) for the observed effect is considered important for developing improved processes for preparation of porous CPP implants as bone substitutes for use in high load-bearing skeletal sites. (c) 2013 Wiley Periodicals, Inc. J Biomed Mater Res Part B: Appl Biomater 00B: 000-000, 2013.

Key Words: porous structures, calcium polyphosphate, sintering, additive manufacturing, bone substitutes, bending strength, nanoindentation testing, elastic modulus

How to cite this article: Hu Y., Shanjani Y., Toyserkani E., Grynpas M., Wang R., Pilliar R.. 2013. Porous calcium polyphosphate bone substitutes: Additive manufacturing versus conventional gravity sinter processing-Effect on structure and mechanical properties. J Biomed Mater Res Part B 2013: 00B: 000-000.

\section{INTRODUCTION}

Development and studies of synthetic porous structures for use in tissue engineering applications including synthetic bone substitutes are currently of great interest. Although autologous bone graft represents the gold standard for replacement of severely traumatized or diseased bone, it is not always available in sufficient quantity or quality. ${ }^{1-3}$ In such cases, alternative bone-substitute materials are required. These include allogeneic bone grafts (allografts) and, less frequently at present, synthetic bone substitutes. $^{1,4,5}$ Synthetic bone substitutes offer an advantage over allografts in that they can be made as required (i.e., off-theshelf products that may exhibit virtually limitless lifetime) and they avoid the need for harvesting tissue from donors and costly processing necessary for reducing the possibility for disease transmission and adverse immunological reaction. If formed as open-pored structures, the porous constructs offer the potential for integration with host bone through bone ingrowth into available porosity. Synthetic bone substitutes preferably should be formed from biodegradable biomaterials so that in time, they may be wholly replaced by healthy natural bone, an obvious advantage. ${ }^{6}$

Correspondence to: R. Pilliar (e-mail: bob.pilliar@utoronto.ca)

Contract grant sponsor: Natural Sciences and Research Council of Canada and the Department of the Army-US Army Medical Research Acquisition Activity (USAMRAA). 
Biodegradable synthetic bone substitutes formed as scaffold-like structures suitable for bone ingrowth and made of organic copolymers (polylactic acid-polyglycolic acid $^{7-9}$ primarily) are currently available and used clinically but these are not ideal for use in high load-bearing skeletal sites due to their relatively low strength and stiffness characteristics. In addition, in vivo degradation of these products with the associated $\mathrm{pH}$ changes that can develop during degradation in vivo ${ }^{10,11}$ can result in unacceptable local inflammatory response.

Previously, we have reported on forming porous calcium polyphosphate (CPP) structures with compressive strength properties that suggested their potential usefulness as bone substitutes in load-bearing skeletal sites (at least where predominantly compressive forces are expected to act). ${ }^{12-14}$ In vivo studies for periods as long as 1 year showed that the porous CPP implants formed by the conventional gravity sintering (CS) method were biocompatible and degraded slowly in vivo with rate of degradation being dependent on the sinter/annealing regime used to form the samples. ${ }^{15,16}$ In vivo studies using CS- and additive manufacturing (AM)formed samples implanted in rabbit femoral condyle sites for 6-week periods and formed using annealing schedules similar to those reported in the present study further confirmed the biocompatible nature of both types of samples and their suitability to allow bone ingrowth as well as their in vivo degradability $(\approx 10 \%$ degradation by 6 weeks in rabbit femoral condyle sites) without invoking an adverse inflammatory response. ${ }^{17}$ Another long-term study involved CS-made porous CPP samples implanted in sheep femoral condyle sites (for up to 9 -month periods). ${ }^{18}$

Our method for forming porous CPP structures involves the use of a 2-step sinter/annealing operation ${ }^{19}$ using a low temperature step 1 anneal in which sinter necks form between amorphous CPP particles via a viscous flow mechanism. ${ }^{20}$ Under appropriate time, temperature, and environment conditions, this results in a porous structure of desired volume percentage of porosity (35\% in this study). Pore size and sinter neck dimensions are determined by CPP particle size and, as noted earlier, temperature, time, and atmosphere used for the step 1 sinter anneal. A subsequent higher temperature anneal (step 2 anneal) results in full crystallization of the initially amorphous CPP as well as growth of crystals within the sintered particles resulting in formation of a higher strength, more stable $\beta$-CPP structure. Little change in pore size or sinter neck geometry occurs after complete crystallization of the CPP (i.e., after the step 1 sinter anneal), due to the slower mass transport kinetics associated with crystalline phase sintering (i.e., volume and grain boundary diffusion mechanisms as opposed to the viscous flow and surface diffusion mechanisms that are primarily responsible for sinter neck formation of amorphous, glassy structures).

In summary, the two methods for forming porous CPP samples, namely gravity sintering of CPP blocks followed by machining of the blocks to form bending test bars (referred to as CS) and AM for layer-by-layer block build-up and annealing followed by machining to form test bars have been studied. Mechanical and physical characteristics including machinability ${ }^{21}$ and in vivo behavior (i.e., biocompatibility, bone ingrowth and in vivo degradation characteristics) of CS- and AM-formed samples have been reported previously. ${ }^{12-17,21}$ In previous studies, uniaxial compression testing demonstrated significantly higher compressive strengths for samples made by AM $(\approx 1.6-2.4$ times greater than CS-made samples). The AM-made samples exhibited anisotropic strength properties, a finding that was attributed to the plate-like CPP particle shape and resultant preferential particle orientation during layer-by-layer sample build-up. ${ }^{14}$ Bone ingrowth characteristics, determined using implants placed in rabbit femoral condyle sites, appeared similar for samples prepared by one or the other method. ${ }^{16,17}$ In vivo degradation characteristics also were not significantly different. ${ }^{17}$

The present study further compares samples made by the two methods for part preparation through evaluation of bending strength (reflective of tensile strength). In addition to generating additional information on mechanical properties, the major objective of the study was to gain an understanding of the reason(s) for the observed higher mechanical properties for samples formed by the AMmethod and to determine whether this suggested strategies for making stronger porous CPP samples in general. Toward this objective, nanoindentation testing for determination of the intrinsic elastic modulus of sintered CPP particles constituting AM and CS test samples was undertaken. Comparison of elastic modulus and X-ray diffraction (XRD) was used to determine whether differences in atomic lattice arrangement (crystallographic structure and interatomic spacing) may have contributed to the observed difference in mechanical properties.

\section{MATERIALS AND METHODS}

\section{Fabrication of Porous CPP Structures}

The methods used to prepare the porous CPP structures both by the AM and CS routes have been described elsewhere. ${ }^{12-14}$ Briefly summarizing, for the AM-made samples, 10 wt \% polyvinyl alcohol (PVA) in particle form $(<60 \mu \mathrm{m}$ size) is mixed with $75-150 \mu \mathrm{m}$ CPP powders and a solvent used to dissolve the PVA, $\left(\mathrm{ZB}^{\mathrm{TM}} 58-\mathrm{Z}\right.$ Corporation, Burlington, MA), is added to each CPP-PVA layer during sample build-up. The dissolved PVA serves as a binder to hold CPP particles together thereby allowing formation of the "green" samples. The packing density of AM-made "green" samples is approximately $40 \%$ of full theoretical CPP density as a result of the counter-rotating roller packing of powders during layer stacking. ${ }^{14}$ Two sets of AM-made samples were machined from sintered CPP block, (1) AM-H in which the stacking layers are parallel to the neutral axis of bending test bars and (2) AM-V in which the stacking layers are perpendicular to the neutral axis of the bars [Figure 1(a)]. The "green" samples are annealed at $400^{\circ} \mathrm{C}$ (in ambient laboratory air atmosphere) for $1 \mathrm{~h}$ to burnout the organic binder and solvent. For CS sample preparation, CPP powders are packed into a Pt mold and vibrated to give a packing density $\sim 50 \%$ of full theoretical CPP density. The AM and CS 


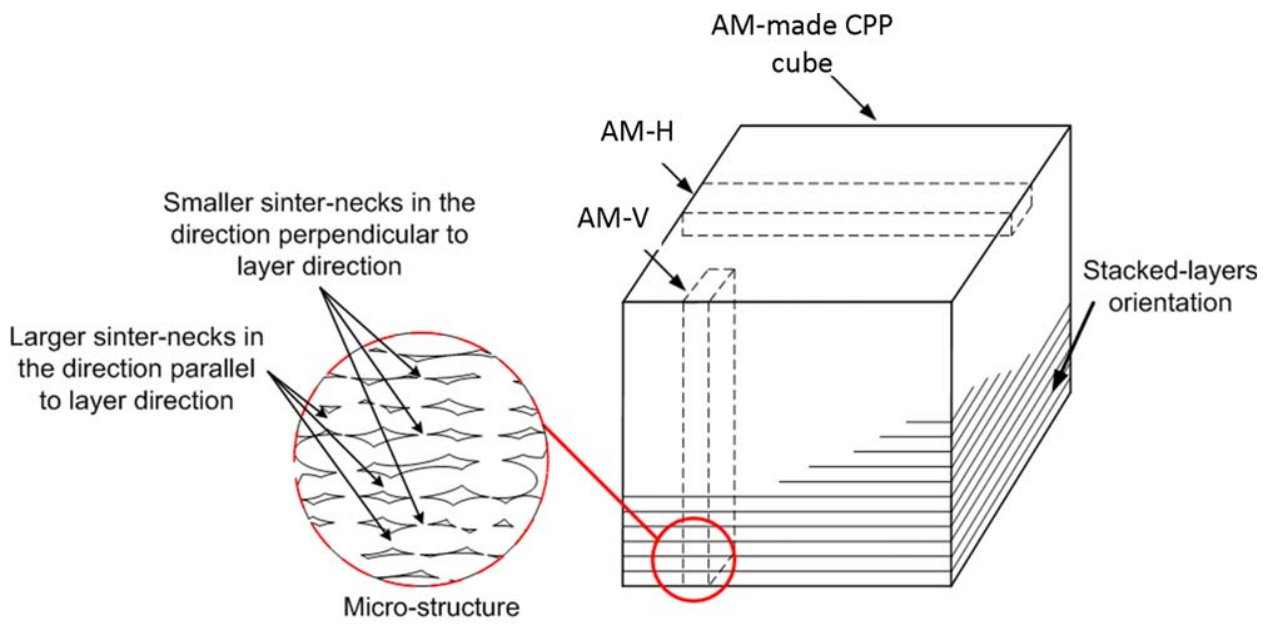

(a)

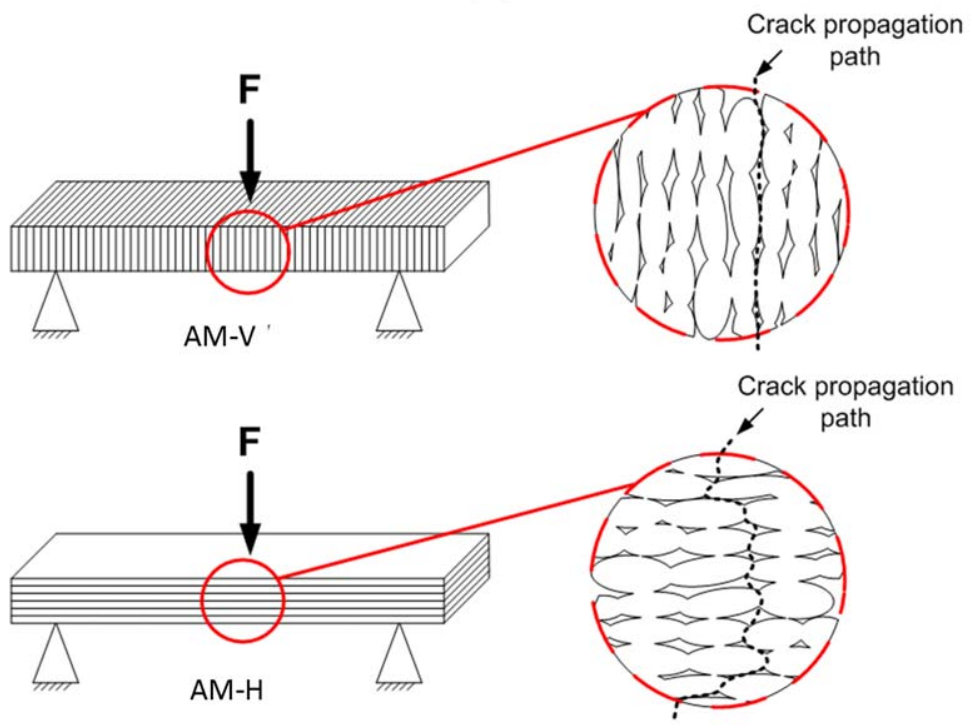

(b)

FIGURE 1. (a) Illustration showing the orientation of AM-H and AM-V bending test samples machined from CPP blocks. (b) For the AM-H samples, three-point loading results in crack propagation primarily through larger sinter necks that form during sintering. This is presumed to be due to the end-to-end contacts of the nonequiaxed CPP particle shape and a preferred orientation resulting form particle lay-up during AM processing. [Color figure can be viewed in the online issue, which is available at wileyonlinelibrary.com.]

samples are then subjected to a 2-step annealing treatment ${ }^{19}$ specifically designed to yield the desired 35 vol \% porosity samples either by CS or AM procedures. The 2-step annealing treatment includes sintering the amorphous CPP particles in a controlled relative humidity (RH) air environment $(\mathrm{RH} \approx 50 \%$ in the present study) for $1 \mathrm{~h}$ at a step 1 temperature above the glass transition temperature $\left(T_{\mathrm{g}} \approx\right.$ $545^{\circ} \mathrm{C}$ ) of the CPP but below the temperature at which rapid crystallization of the amorphous CPP will occur. This results in rapid sinter neck formation by a viscous flow mechanism $^{20}$ as well as the initiation and progression of crystallization of the CPP. A second higher temperature anneal at $950^{\circ} \mathrm{C}$ for $1 \mathrm{~h}$ is used to ensure full crystallization. It was determined through preliminary trial treatments that a higher step 1 sinter temperature is required for the AM- made samples to achieve the same final volume percentage of porosity as the CS-formed samples ( $\sim 35 \mathrm{vol} \%)$. In the present study, the CS samples were annealed at $585^{\circ} \mathrm{C}$, for 1 $\mathrm{h}$ during the step 1 sinter anneal, whereas a $628^{\circ} \mathrm{C}$, 1 -h anneal was used for the AM-formed samples.

\section{Assessment of Sintered Structures}

The sintered samples were examined visually and by scanning electron microscopy (secondary electron imaging, Hitachi S2500 microscope-electron beam acceleration voltage $=10$ $\mathrm{kV}$ or $20 \mathrm{kV}$ ). In addition to examining the surface appearance to view the porous structure of the samples, one test sample of each type was examined by scanning electron microscopy (secondary electron imaging) after testing to failure to view the features and images of a typical fracture region. These 
TABLE I. Mechanical Test results

\begin{tabular}{lccc}
\hline & CS & AM-H & AM-V \\
\hline Elastic constant (GPa) & $6.37 \pm 0.81$ & $14.73 \pm 1.31$ & $12.23 \pm 1.86$ \\
Bending strength (MPa) & $13.72 \pm 2.76$ & $20.13 \pm 3.36$ & $16.38 \pm 2.54$ \\
Characteristic bending strength (MPa) & 15.17 & 21.78 & 17.51 \\
Weibull modulus & 4.23 & 5.6 & 6.88 \\
Compressive strength (MPa; from Ref. 17) & $21.06 \pm 4.51$ & $50.17 \pm 4.74$ & $33.86 \pm 6.32$ \\
Weibull modulus (from Ref. 17) & 4.45 & 3.93 & 5.23 \\
Porosity (\%) & $34.9 \pm 0.9$ & $36.4 \pm 2.2$ & $36.1 \pm 2.2$ \\
\hline
\end{tabular}

images were used, as described in Results section, to estimate the relative size of sinter necks and other features that might have influenced sample strength. Normal methods (i.e., addition of a thin Au conducting film) were used for preparing samples for SEM examination.

\section{XRD Analysis}

XRD analysis was used (SA-HF3, Rigaku, Japan) to characterize the crystallography of the sintered CPP samples. A step scan of $2 \theta=0.02^{\circ}$ between $10^{\circ}$ and $60^{\circ}$ was used. For the XRD assessment, the samples were crushed to a fine powder with a Agate mortar and pestle.

\section{Bending Tests}

Bending test bars $[2.0 \mathrm{~mm}$ width $\times 1.5 \mathrm{~mm}$ thickness $\times 25.0$ $\mathrm{mm}$ length-Figure 1(b)] were machined from $25 \mathrm{~mm} \times 20$ $\mathrm{mm} \times 10 \mathrm{~mm}$ blocks of sintered CPP made either by CS or AM [Figure 1(a)]. The AM-made bending test bars were machined from the blocks with the particle layers due to sample build-up either parallel (AM-H) or perpendicular (AM-V) to the length of the bars [Figure 1(b)]. The three-point bend tests conformed to ASTM C1161, sample size A specifications. $^{22}$

For the bending tests, 10 samples of each type were used. The test bars were loaded using the servohydraulic mechanical testing machine at a crosshead speed of $0.2 \mathrm{~mm}$

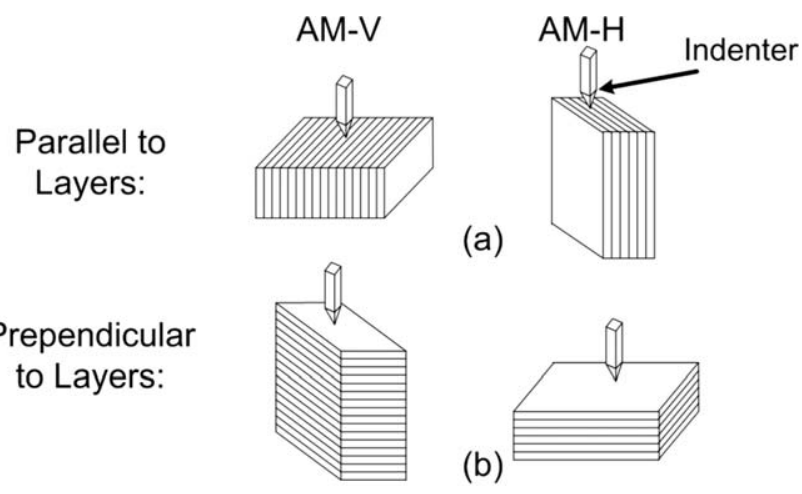

\section{No-layered structure:}

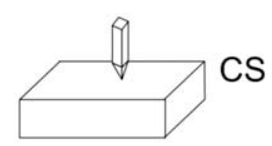

(c)

FIGURE 2. Schematic of the nanoindentation process: $A M-V$ and $A M-H$ samples were tested in both parallel and perpendicular directions to the stacked-layers. $\min ^{-1}$. The bending elastic constant was determined from the slope (within the constant slope region) of the loaddeflection curve using the following relation:

$$
E=\frac{L^{3} \cdot m}{4 \cdot b \cdot d^{3}}
$$

where $L$ is the support span (20 mm), $m$ is the slope of the load-displacement curve (in $\mathrm{N} \mathrm{mm}^{-1}$ ), and $b$ and $d$ are specimen width and thickness (in $\mathrm{mm}$ ), respectively. The characteristic bending strength and Weibull modulus were determined using 10 samples of each sample type. Included in Table I are the mean percent densities (relative to full theoretical density) of the CPP test bars.

For the nanoindentation determinations, samples cut from the test bars were embedded in EpoThin low viscosity epoxy resin (Buehler, Lake Bluff, IL) and polished with $1 \mu \mathrm{m}$ diamond suspension (Buehler) followed by mechanical vibration polishing using $0.05 \mu \mathrm{m}$ colloidal silica suspension polishing medium. The samples were oriented relative to the indenter loading direction with stacking layers resulting from the AM build-up either parallel (AM-H) or perpendicular (AM-V) to the sample axes. These two sample types were cut and polished (see below) to allow nanoindenter loading either parallel or perpendicular to the layers (Figure 2). The CS sample is also shown in the figure.

A commercial nanoindentation test system (Nano Indenter XP System; MTS Nano Instruments, Oak Ridge, TN) equipped with a Berkovich diamond tip (AccuTip) was used to evaluate the modulus of elasticity of CPP sintered particles forming the three sample types using the continuous stiffness measurement mode during nanohardness indentation testing. $^{23,24}$ The amplitude and frequency of the sinusoidal signal was $2 \mathrm{~nm}$ and $45 \mathrm{~Hz}$, respectively. The measurements were displacement controlled with penetration depth of $2000 \mathrm{~nm}$ for dry conditions. The strain rate was set at $0.05 \mathrm{~s}^{-1}$. The modulus of elasticity was calculated from load-deflection slopes at $1000 \mathrm{~nm}$ (i.e., between $500 \mathrm{~nm}$ and $1500 \mathrm{~nm}$ penetration depth). Ten indentations were made on each sample. For the AM samples, the nanoindentation test was done in two planes: (1) across the radial sections and (2) along the longitudinal sections of the samples (Figure 2).

\section{Statistical Analysis}

One-way analysis of variance was used to compare modulus of elasticity values determined from the nanoindentation load-displacement curves with significant differences being 


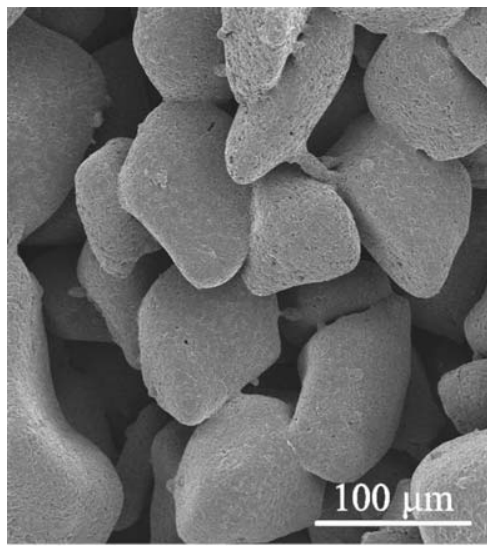

(a)

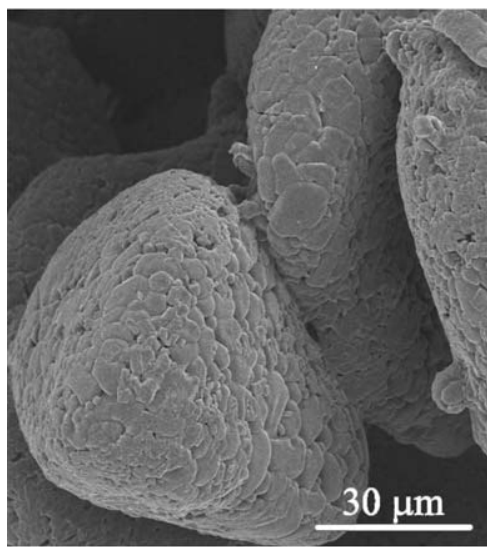

(d)

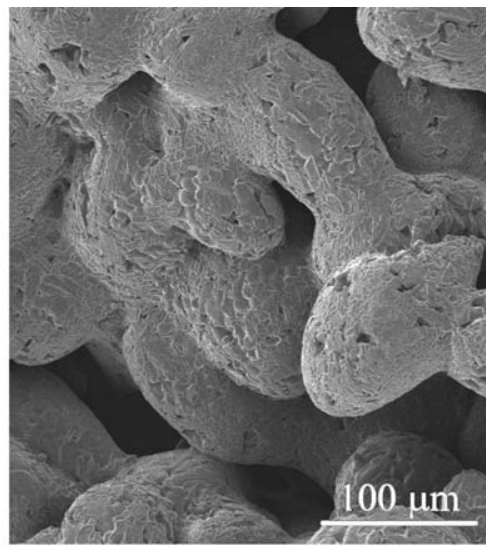

(b)

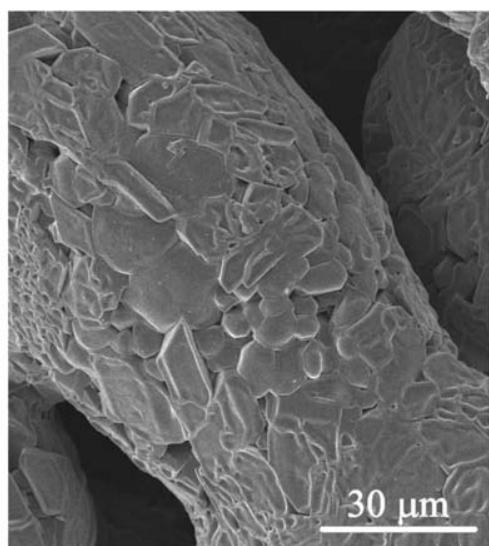

(e)

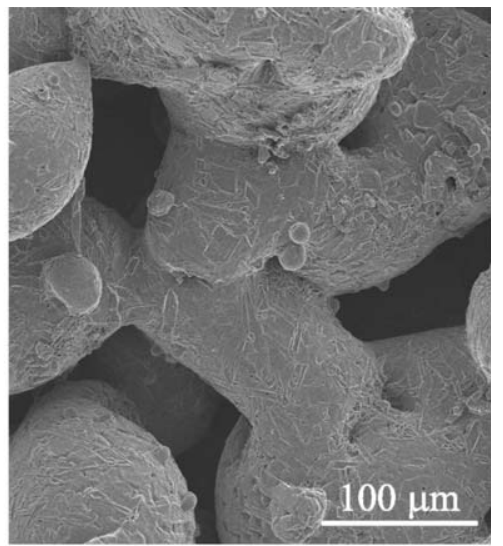

(c)

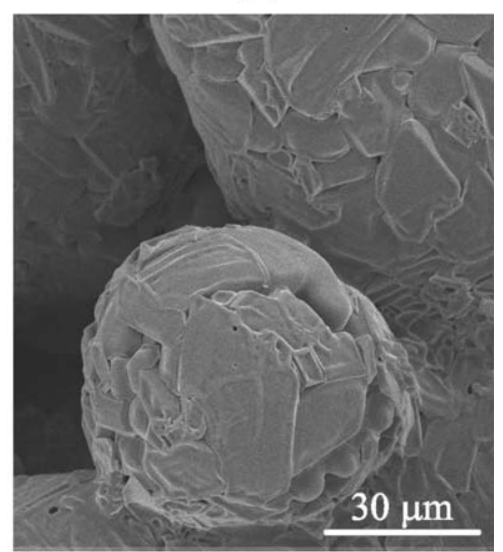

(f)

FIGURE 3. Low and high magnification SEM images of as-made porous CPP samples; (a and d) CS samples, (b and e) AM-H samples, and (c and f) AM-V samples. The more extensive sinter neck formation for the SFF-made samples is evident in the lower magnification images, whereas the higher magnification images show the larger grains within the sintered CPP particles for the SFF-made samples.

assigned for $F$ values greater than $F_{\text {critical }}$ corresponding to $p<0.05$. For the bending tests, Weibull analysis based on results from 10 samples per test group was used for determination of characteristic strength and Weibull modulus.

\section{RESULTS}

\section{Porosity, Sinter Neck Characteristics and XRD Analysis}

The average volume percent porosities of the AM-V, AM-H, and CS samples were all at the desired level $(\approx 35$ vol \%Table I). XRD spectra for CS- and AM-made samples were the same as previously reported indicating the presence of $\beta$-CPP phase only. ${ }^{13}$ SEM images (Figure 3 ) indicated that average pore size and sintered particle size were greater for the AM samples. This was consistent with the microcomputed tomography assessment presented previously. ${ }^{13}$ The higher magnification images [Figure $3(d-f)$ ] also showed coarser crystals (grains) within the sintered particles of the AM-made samples. Also evident are finer micron and submicron sized voids, primarily intergranular, within the particles of all three sample types. These intergranular voids appear coarser in the AM-made samples.

Scanning electron micrographs of representative fracture surfaces of tested samples are shown in Figure 4. For all samples, as expected, fracture occurred by cracks propagat- ing through sinter neck regions since these represent regions of higher stress by virtue of their limited crosssectional dimension and the stress concentration associated with the radii of curvature of the sinter necks. The fracture areas highlighted by the Bitmap images [Figure 4(d-f)] illustrate more substantial fractured areas (and, hence, sinter necks) associated with the AM samples.

\section{Sample Appearance}

The "green" samples appeared white [Figure 5(a)]. After the polymer burnout treatment $\left(400^{\circ} \mathrm{C}\right.$ for $\left.1 \mathrm{~h}\right)$, the AM samples appeared black, presumably a result of carbon remaining after the polymer burnout [Figure 5(b)]. The samples retained a grayish appearance after the step 1 sinter anneal [Figure 5(c)] but after the higher temperature step 2 treatment $\left(950^{\circ} \mathrm{C}, 1 \mathrm{~h}\right.$ anneal in air), the $\mathrm{AM}$ samples appeared white [Figure $5(\mathrm{~d})$ ] as the carbon residue was presumably oxidized and lost as $\mathrm{CO}_{2}$ with only some very fine dark specks remaining on the surface after the anneal. The CS samples appeared white at all stages of the sinter anneal treatment.

\section{Mechanical Properties}

Elastic constants, characteristic Weibull bending strengths and Weibull moduli determined for the three sample types 


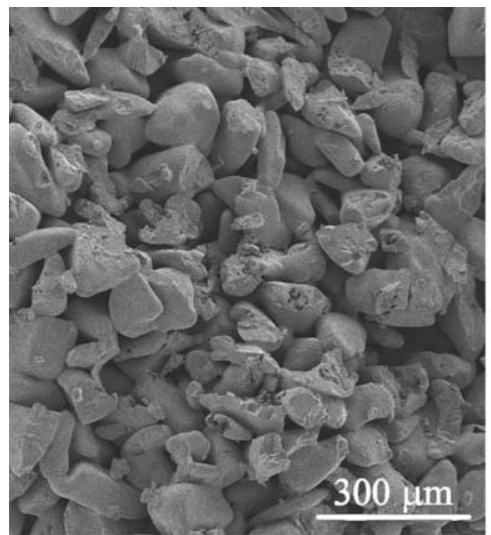

(a)

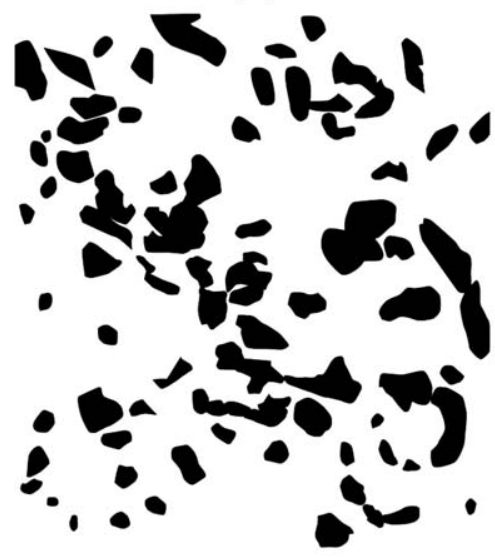

(d)

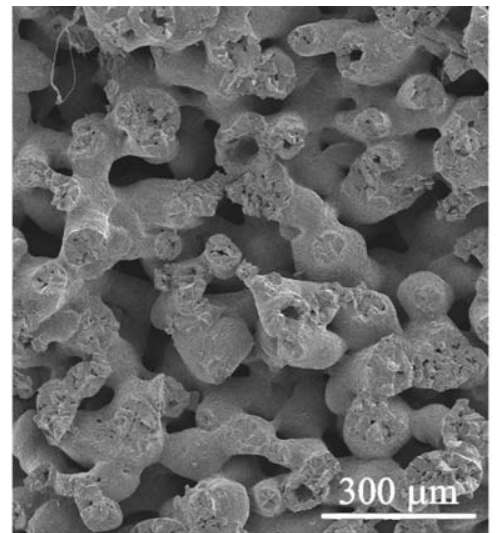

(b)

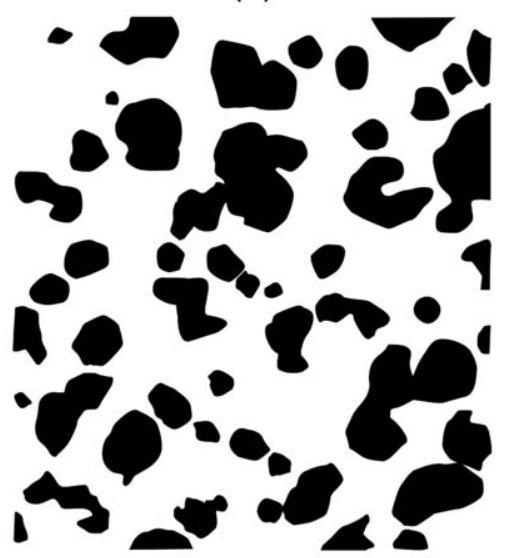

(e)

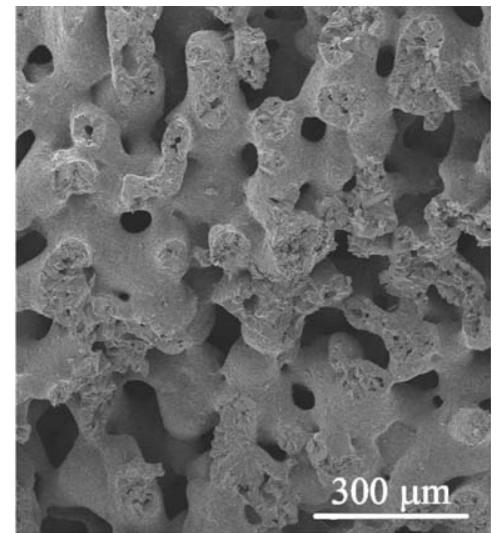

(c)

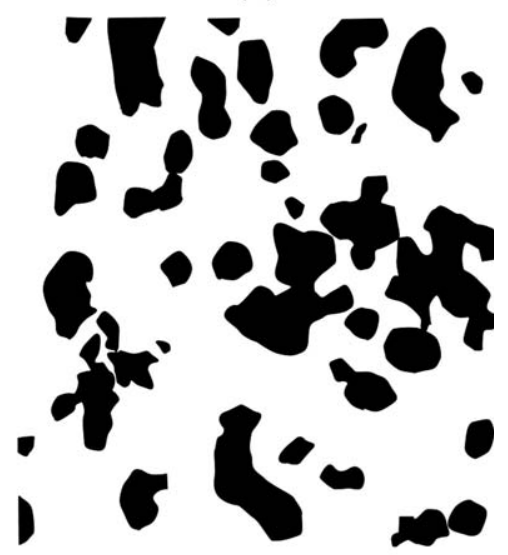

(f)

FIGURE 4. SEM images of fracture surfaces of bending test samples (a) CS, (b) AM-H, and (c) AM-V. Bitmap images highlighting the corresponding sinter neck fracture regions; (d) CS-percentage of fractured sinter neck $\approx 24 \%$, (e) AM-H-percentage of fractured sinter neck $\approx 36 \%$, and (f) SFF-V-percentage of fractured sinter neck $\approx 30 \%$.

(CS, AM-H, and AM-V) are listed in Table I. Also included in the table are the previously reported uniaxial compression test results. ${ }^{13,14}$ As shown, the elastic constant is greatest

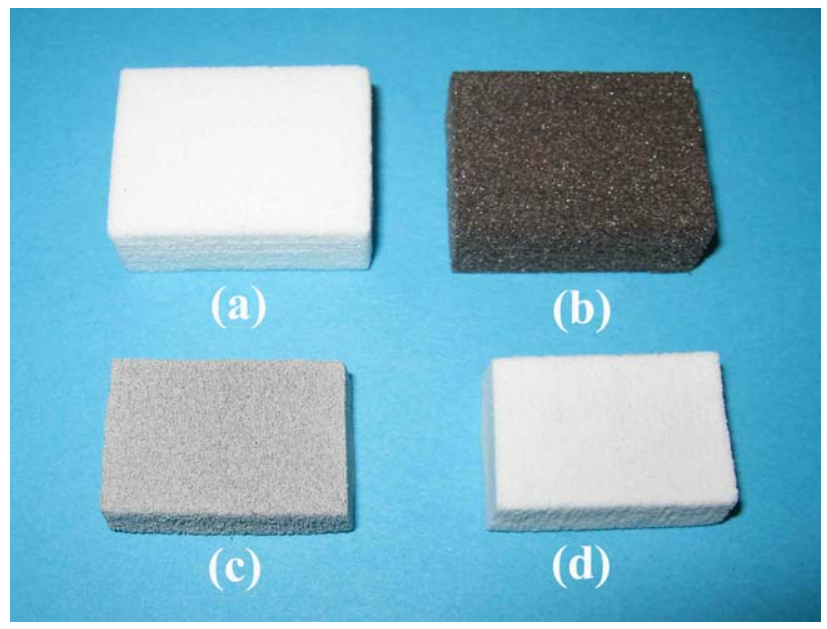

FIGURE 5. Images of AM-made samples after different stages of preparation; (a) "green" sample after AM CPP powder layering process, (b) after polymer-burnout treatment at $400^{\circ} \mathrm{C}$, (c) after step 1 sinter anneal at $628^{\circ} \mathrm{C}$, and (d) after final $950^{\circ} \mathrm{C}$ anneal. [Color figure can be viewed in the online issue, which is available at wileyonlinelibrary.com.] for the AM-made samples $(14.73 \pm 1.31 \mathrm{GPa}$ and $12.23 \pm$ $1.86 \mathrm{GPa}$ for the AM-H and AM-V samples, respectively, compared with $6.37 \pm 0.81 \mathrm{GPa}$ for the CS samples). The AMmade samples also display higher characteristic bend strength as well as higher Weibull modulus indicating their more reproducible bending strengths.

Typical load-displacement curves for nanoindentation testing are shown in Figure 6. The average elastic moduli determined from these curves based on the slope corresponding to measurements at a depth of 500-1500 nm for the three sample types are presented in Table II. Statistical analysis indicated no significant difference in elastic modulus between sample types $(\mathrm{p}>0.05)$. The values for the different sample types and two indenter loading directions (for the AM samples) are virtually the same for all samples $(E \approx 64 \mathrm{GPa})$.

\section{Fracture Appearance}

SEM examination of the fracture surfaces indicated failure predominantly through the sinter neck regions for all samples. The percent fracture area (and, hence, the approximate sinter neck area) was measured from the Bitmap images depicted in Figure 4(d-f). Although only one or two samples of each type were included in this assessment, differences were apparent between the CS and AM samples. The 


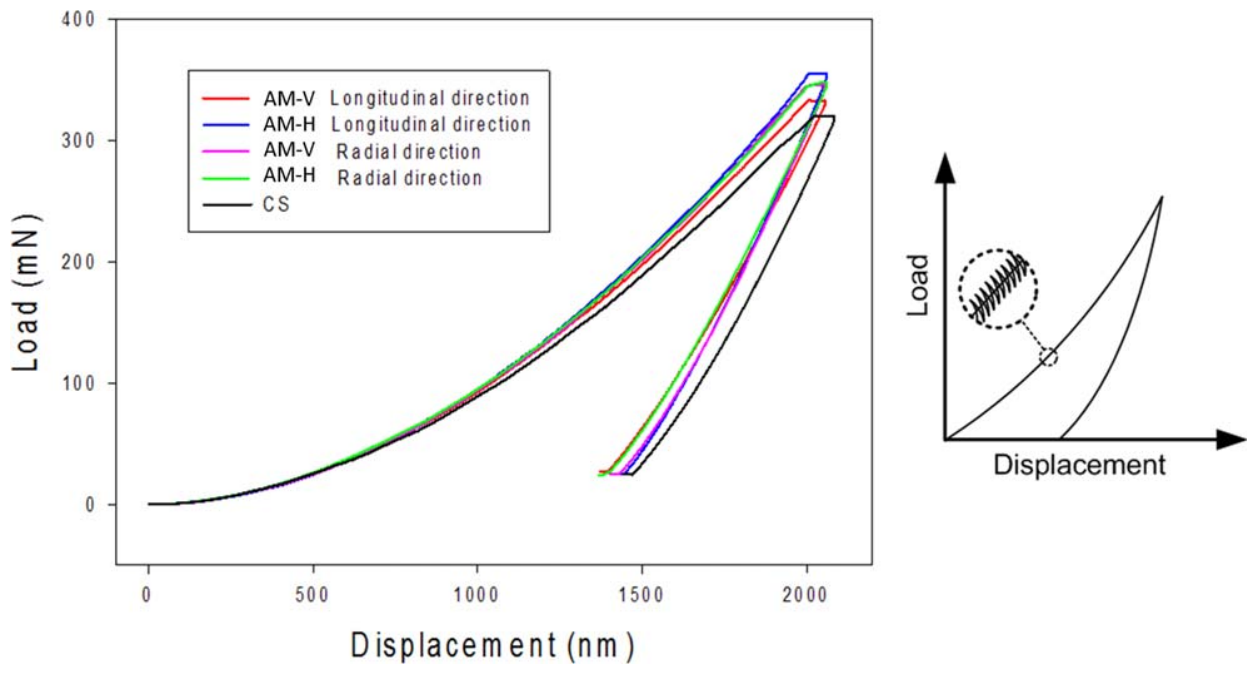

FIGURE 6. Typical load-displacement curves of nanoindentation on AM-V, AM-H, and CS CPP samples. Young's moduli of samples were calculated through the slope of the unloading curve. [Color figure can be viewed in the online issue, which is available at wileyonlinelibrary.com.]

greatest percent fracture area corresponded to the AM-H sample $(\sim 36 \%)$ followed by the AM-V sample $(\sim 30 \%)$ with the CS sample displaying the smallest sinter neck fracture area $(\sim 24 \%)$. Within the limits of this study (i.e., only one or two samples analyzed per sample type), the results suggest a correlation between percent fractured sinter neck area and mechanical properties with the lowest bending strength and elastic constant for the CS samples.

\section{DISCUSSION}

Open-pored CPP structures can be made either by sintering CPP blocks from which appropriate forms can be machined or by additive manufacture plus sinter annealing of final or near-final forms. With the latter technique, minimal final finish machining might be required in some cases. Although minimal strength criteria for bone substitutes remain poorly defined, initial compressive strength $\geq 40 \mathrm{MPa}$ and bending strength $\geq 15 \mathrm{MPa}$, respectively, is expected to satisfy most situations. This is based on the reported range of compressive strengths for cancellous and cortical bone (i.e., 1-10 $\mathrm{MPa}$ for human cancellous bone and up to $150 \mathrm{MPa}$ for cortical bone $\mathrm{e}^{25}$ and limited loading imposed during the early healing phase when some bone ingrowth will occur. Higher initial strengths of porous CPP implants would offer an obvious advantage suggesting a preference for implants made by the AM method, based on the results of the present bending strength and previously reported compressive strength determination. It should be noted that as bone

TABLE II. Modulus of Elasticity, E, for CS Samples and AM-H and AM-V Samples With Loading Parallel and Perpendicular to the Stacked Layers of the AM Samples

\begin{tabular}{lccc}
\hline & AM-H, & AM-V, & CS, \\
& $E(\mathrm{GPa})$ & $E(\mathrm{GPa})$ & $E(\mathrm{GPa})$ \\
\hline $\begin{array}{l}\text { Parallel to layer } \\
\text { Perpendicular to } \\
\text { layer }\end{array}$ & $66.37 \pm 4.09$ & $62.88 \pm 3.71$ & $63.88 \pm 5.04$ \\
& & & \\
\hline
\end{tabular}

grows into the porous structures, higher strength composite structures effectively develop thereby further supporting higher, longer term load-bearing requirements. The ultimate goal is the complete replacement of the synthetic biodegradable bone-substitute system by natural bone. An appropriate degradation rate for this to occur while avoiding implant fracture is essential. The slow in vivo degradation rates characterizing the porous CPP structures made for this study and as described in Ref. 17 should satisfy this condition. Further in vivo studies with porous CPP implants placed in more severe load-bearing sites are presently in progress. Studies also demonstrated a higher Weibull modulus for AM-made samples suggesting better reproducibility of implants so made.

The difference in bending strengths and elastic constants between AM and CS samples determined in the present study, as well as compressive strengths as previously reported, ${ }^{13,14}$ is believed to be due to sample structural differences rather than material property differences. This is supported by the observation of similar elastic moduli, (an intrinsic material property), as determined from results of nanoindentation testing for AM- and CS-made samples as well as by the similar XRD spectra observed for samples made by the two methods. The observed difference in sinter neck size and form for AMmade compared with CS-made samples (Figures 3 and 4) is considered the reason for the observed difference in strength and elastic constant properties.

As stated in Introduction section, a major objective of the current study was to determine whether differences in processing were responsible for the observed results. Our hypothesis is that the difference in sinter neck size for samples made by AM and CS methods is due to the different step 1 sintering temperatures used during processing to form the $35 \mathrm{vol} \%$ porous structures. A higher step 1 sinter anneal to form 35 vol \% AM-made samples compared with the $\mathrm{CS}$ samples, $\left(628^{\circ} \mathrm{C}\right.$ cf. $\left.585^{\circ} \mathrm{C}\right)$, was determined empirically. Two possible reasons for the need for a higher step 1 sinter anneal for the AM-made samples and how this could 
have resulted in the structural and mechanical property differences are proposed:

1. According to the theory of glass material sintering, ${ }^{26,27}$ the porosity after sintering of CPP amorphous/glassy powders, $V_{\mathrm{p}}$, is determined by the "green" pore fraction, $V_{\mathrm{p} 0}$ (which is inversely related to the packing density of the CPP powders), according to the following relation:

$$
\log \left(\frac{V_{p}}{V_{P_{0}}}\right)=-\frac{\gamma \cdot t}{D \cdot \eta}
$$

where $\gamma$ is the surface energy, $D$ is the particle size, $t$ is the sintering time, and $\eta$ is the viscosity of the glassy phase.This suggests that a lower packing density (i.e., higher $V_{\mathrm{p} 0}$ ) will result in a lower sintered density (i.e., higher $V_{\mathrm{p}}$ ) after sintering at a given step 1 temperature for a fixed time. In our studies, it was observed that the packing density of "green" block samples made by CS methods were approximately $10 \%$ greater than those made using the AM process (i.e., $50 \%$ vs. $40 \%$ ). Thus, according to the relation shown in Eq. (2) a $585^{\circ} \mathrm{C}$, 1-h step 1 sinter anneal would be expected to yield lower density AM samples compared with CS samples sintered under the same temperature and time conditions. To achieve the desired $35 \mathrm{vol} \%$ porosity would require the use of a higher step 1 one-h sinter anneal for AM-made samples. Empirically, we determined that a $628^{\circ} \mathrm{C}$ step 1 sinter temperature satisfied this requirement. (It should be noted that the rate of heating to the $628^{\circ} \mathrm{C}$ holding temperature is also important since viscous flow, as well as initiation of crystallization, occurs during this heating to the hold temperature). The viscosity of the glassy CPP phase present in the 550$628^{\circ} \mathrm{C}$ range is not known but a lower viscosity is expected at higher temperatures since viscosity is related to temperature by the following relation:

$$
\eta=\eta_{0} \cdot \exp \left(\frac{Q}{k \cdot T}\right)
$$

where $Q$ is an activation energy, $\eta_{0}$ a proportionality coefficient, and $T$ the absolute temperature. The activation energy, $Q$, associated with the step 1 sintering of CPP powders was determined in earlier studies to be a function of $\mathrm{RH}$ during sintering and for the $50 \% \mathrm{RH}$ atmosphere used to prepare the samples in the present study, $Q \approx 60-70 \mathrm{~kJ} \mathrm{~mol}^{-1}{ }^{20}$ (This value is consistent with a viscous flow transport mechanism during sintering in the $550-650^{\circ} \mathrm{C}$ temperature range.) Thus, from Eq. (3), the higher step 1 anneal used with the AM samples results in greater viscous flow leading to the formation of larger sinter necks, as was observed in this study. The time to reach the step 1 sinter temperature during heating samples to the $628^{\circ} \mathrm{C}$ temperature further contributes to greater sinter neck growth.

2. A second possible reason for the need for a higher step 1 sinter temperature to achieve the desired density for the AM-made samples is related to surface modification of the amorphous CPP particles resulting in a reduction in surface energy. The dark appearance of the AM-made samples after the $400^{\circ} \mathrm{C}$ burnout treatment [Figure 5(b)] suggests the presence and retention of an adsorbed carbon deposit on the particle surfaces resulting in a lower surface energy since otherwise the carbon deposit would be thermodynamically unstable and would not be retained. A lower surface energy reduces the driving force for sintering [Eq. (2)] thereby necessitating the use of a higher sintering temperature (or longer sintering time) to achieve desired volume percentage of porosity.

Whether one or both of these factors contributed to the observed need for the higher step 1 sinter temperature with the AM samples, larger sinter necks formed giving greater strength and stiffness for the AM-made samples. In addition to the choice of the step 1 sinter temperature, once a temperature above the glass transition temperature $\left(T_{\mathrm{g}} \approx 500^{\circ} \mathrm{C}\right.$ for amorphous (PP) is reached, annealing rate to reach the step 1 sintering temperature is important since the processes of viscous flow and crystal nucleation and growth both occur during heating to the step 1 temperature. The more rapid sinter neck development associated with viscous flow only occurs to the point of full CPP crystallization at which time significantly slower transport mechanisms (volume and grain boundary diffusion) predominate. In our studies, temperature was raised at a fixed rate $\left(5^{\circ} \mathrm{C} \mathrm{min}^{-1}\right)$ from approximately $550^{\circ} \mathrm{C}$ (i.e., above $T_{\mathrm{g}}$ but where the existence of the amorphous CPP phase was confirmed) to the final step 1 temperature. Other factors including particle size and distribution, particle shape and furnace atmosphere used during powder preparation and sintering) also can affect the sintering process so that, currently, processing parameters need to be determined empirically to define the conditions for forming a specific sample shape and size. Currently, studies are in progress to determine whether one or the other of the suggested reasons (i.e., different packing density or surface energy modification) is more important in affecting the required step 1 sintering temperature. In addition, preliminary studies (unpublished) suggest that modifying CS (CS) processes by the addition of an organic phase, as for the AM-made samples, can yield a similar benefit.

The anisotropic strength properties of the AM-made samples (i.e., higher compressive strength for AM-H compared with AM-V samples as reported previously ${ }^{14}$, are also reflected in bending strength properties (Table I), although not to as great a degree as for compressive strength (i.e., $24 \%$ difference for the bending strengths compared with $48 \%$ for the compressive strengths). The difference between bending strengths of AM-made samples compared with CS samples is also not as great as for compressive strength. This reflects the inherent fracture characteristics of ceramics namely their greater susceptibility to crack initiation at stress concentration sites and easier crack propagation when loaded in tension or shear compared with loading in compression. The slightly greater bending strength for the AM-H samples is a consequence of either larger sinter necks resisting crack propagation in directions normal to the particle layers, (as discussed in a previous report ${ }^{14}$ ), or greater 
resistance to crack propagation in this direction due to a more tortuous propagation route [see illustration in Figure 1(b)]. Although this may also be so for the CS samples, their smaller sinter neck sizes appear to dominate, resulting in lower bending strengths regardless of the crack propagation characteristics.

Consideration was given to the possibility that differences in the number or size of structural flaws or defects due to $\mathrm{CS}$ and AM processing might have caused the observed differences in strength. As noted, in addition to the macroscopic pores, (larger for the AM samples), smaller intergranular micron- and submicron-sized voids were seen in all samples (again somewhat larger for the AM samples). Although the voids and surface discontinuities would act as stress concentrators, these are expected to affect both sample types more or less equally.

Elastic constants (Table I) and the estimates of sinter neck area [Figures $4(\mathrm{~d}-\mathrm{f})$ ] suggest a relation between bending elastic constant and sinter neck area. This is consistent with the premise that mechanical properties for the porous CPP samples of fixed volume percentage of porosity are determined by the product of size and number of sinter necks (equal to total sinter neck surface area) along the fracture path. The larger differences between AM and CS samples for elastic constants $(131 \%$ and $91 \%$ for AM-H and $\mathrm{AM}-\mathrm{V}$, respectively, compared with CS samples) in comparison with the strength differences is due to the fact that the elastic properties are influenced to a lesser degree by flaws and stress concentrators. The elastic constant is determined by material elastic modulus and sample geometry (both external form and internal architecture for porous structures). In view of the fact that the modulus of elasticity was not significantly different for CS- and AM-made samples, structural differences (geometry) appear to be the factor that determines the elastic constant.

The nanoindentation tests and estimates of intrinsic CPP elastic properties (elastic modulus) represent a preliminary study undertaken to determine this property for our CPP samples to gain a better understanding of the observed differences in strength and elastic behavior. It resulted in an estimate of Modulus of Elasticity for $\beta$-CPP. To our knowledge, this property has not been reported previously. The value determined $(\approx 64 \mathrm{GPa})$ is similar to that reported in another study of elastic modulus of amorphous CPP fibers $(67.5 \mathrm{GPa}){ }^{28}$

\section{CONCLUSIONS}

The results of this study suggest that the higher elastic constant (effective elastic modulus), bending strength (and compressive strength) of porous CPP samples made by AM compared with those formed using CS and machining procedures is a result of structural differences related to sinter neck size that form during processing. Porous CPP structures are formed using a 2-step sinter/anneal procedure with the volume percentage of porosity being determined by sintering of amorphous CPP particles at a step 1 sinter anneal allowing sinter neck formation by a viscous flow mechanism. The need for and use of different temperatures for this step in sintering CPP particles to form the porous structures is proposed as the cause of the larger sinter neck sizes for the AM-made samples. Two possible reasons are proposed for this required change in the step 1 sinter temperature namely different initial packing densities for the CS- and AM-made "green" sample blocks and/or lower surface energy due to an adsorbed carbon-based deposit on the CPP particles of the AM-made samples before the step 1 sinter treatment. The present study suggests an advantage of AM for forming porous CPP bone-substitute implants where higher strengths may be required. Modification of CS processes to achieve the same benefits based on the findings may be possible.

\section{REFERENCES}

1. Bauer TW, Muschler GF. Bone graft materials. An overview of the basic science. Clin Orthop Relat Res 2000;371:10-27.

2. Murray JE. The first successful organ transplants in man. J Am Coll Surg 2005;200:5-9.

3. Leon-Villapalos J, Eldardiri M, Dziewulski $P$, The use of human deceased donor skin allograft in burn care. Cell Tissue Bank 2010; 11(1):99-104.

4. Hollinger JO, Brekke J, Gruskin E, Lee D. Role of bone substitutes. Clin Orthop 1996;324:55-65.

5. Damien CJ, Parsons JR. Bone graft and bone graft substitutes: A review of current technology and applications. J Appl Biomater 1991;25(3):187-208.

6. Bohner M. Resorbable biomaterials as bone graft substitutes. Mater Today 2010;13(1-2):24-30.

7. Thomson RC, Yaszemski MJ, Powers JM, Mikos AG. Fabrication of biodegradable polymer scaffolds to engineer trabecular bone. J Biomater Sci Polym Ed 1995;7(1):23-38.

8. Wu YC, Shaw SY, Lin HR, Lee TM, Yang CY. Bone tissue engineering evaluation based on rat calvaria stromal cells cultured on modified PLGA scaffolds. Biomaterials 2006;27(6):896-904.

9. Athanasiou KA, Agrawal CM, Barber FA, Burkhart SS. Orthopaedic applications for PLA-PGA biodegradable polymers. Arthroscopy 1998;14(7):726-737.

10. Friden T, Rydholm U. Severe aseptic synovitis of the knee after biodegradable internal fixation. A case report. Acta Orthop Scand 1992;63:94.

11. Tegnander A, Engebretsen L, Bergh K, Eide E, Holen KJ, Iversen OJ. Activation of the complement system and adverse effects of biodegradable pins of polylactic acid (Biofix) in osteochondritis dissecans. Acta Orthop Scand 1994;65:472.

12. Pilliar RM, Filiaggi MJ, Wells JD, Grynpas MD, Kandel RA. Porous calcium polyphosphate scaffolds for bone substitution applications - In vitro characterization. Biomaterials 2001;22:963-972.

13. Shanjani Y, De Croos JNA, Pilliar RM, Kandel RA, Toyserkani E. Solid freeform fabrication and characterization of porous calcium polyphosphate structures for tissue engineering purposes. J Biomed Mater Res B: Appl Biomater 2010;93B(2):510-519.

14. Shanjani Y, Hu Y, Pilliar RM, Toyserkani E. Mechanical characteristics of solid-freeform-fabricated porous calcium polyphosphate structures with oriented stacked layers. Acta Biomater 2011;7(4): 1788-1796.

15. Grynpas MD, Pilliar RM, Kandel RA, Renlund R, Filiaggi M. Porous calcium polyphosphate scaffolds for bone substitute applications-In vivo studies. Biomaterials 2002;23(9):2063-2070.

16. Pilliar R, Kandel R, Grynpas M, Hu Y. Porous calcium polyphosphates as load-bearing bone substitutes: In vivo study. J Biomed Mater Part B: Appl Biomater 2013;101(1):1-8.

17. Shanjani $Y, H u$ Y, Toyserkani E, Grynpas M, Kandel RA, Pilliar RM. Solid freeform fabrication of porous calcium polyphosphate structures for bone substitute applications: In vivo studies. J Biomed Mater Part B: Appl Biomater 2013;101(6):972-980.

18. Kandel RA, Grynpas M, Pilliar R, Lee J, Wang J, Waldman S, Zalzal P, Hurtig M. Repair of osteochondral defects with biphasic 
cartilage-calcium polyphosphate constructs in a sheep model. Biomaterials 2006;27:4120-4131.

19. Pilliar RM, Hong J, Santerre PJ. Method of manufacture of porous inorganic structures. US 7494614; 2009.

20. Filiaggi M, Pilliar RM, Hong J. On the sintering characteristics of calcium polyphosphates. Key Eng Mater 2001;192-195:171174.

21. Rouzrokh A, Wei CYH, Erkorkmaz K, Pilliar RM. Machining porous calcium polyphosphate implants for tissue engineering applications. Int J Autom Technol 2010;4(3):291-302.

22. ASTM Standard D790-10. Standard Test Methods for Flexural Properties of Unreinforced and Reinforced Plastics and Electrical Insulating Materials. West Conshohocken, PA: ASTM International; 2011. pp 150-158.
23. Li W, Bhushan B. A review of nanoindentation continuous stiffness measurement technique and its applications. Science 2002; 48(1):11-36.

24. Oliver WC, Pharr GM. An improved technique for determining hardness and elastic modulus using load and displacement sensing indentation experiments. J Mater Res 1992;7(6):1564-1583.

25. Carter DR, Hayes WC. Bone compressive strength: The influence of density and strain rate. Science 1976;194:1174.

26. German RM. Sintering Theory and Practice. New York: John Wiley \& Sons, Inc.; 1996.

27. Zagar L. Theoretical aspects of sintering glass powders. In: M. Ristic, editor. Sintering: New Developments. New York: Elsevier; 1979. pp 57-64.

28. Chang $\mathrm{Q}$, Shi ZL, Li CA. Studies on degradable calcium polyphosphate fibers. Chin J Environ Sci 1997;02:52-57. 


\title{
Experimental characterization and numerical modeling of a micro-syringe deposition system for dispensing sacrificial photopolymers on particulate ceramic substrates
}

\author{
Mihaela Vlasea, Ehsan Toyserkani* \\ Department of Mechanical and Mechatronics Engineering, University of Waterloo, 200 University Avenue West, Waterloo, ON N2L 3G1, Canada
}

\section{A R T I C L E I N F O}

\section{Article history:}

Received 20 November 2012

Received in revised form 1 April 2013

Accepted 12 May 2013

Available online $\mathrm{xxx}$

\section{Keywords:}

Additive manufacturing

Micro-syringe deposition

Fluid dispensing

Particulate substrate

\begin{abstract}
A B S T R A C T
This work addresses the characterization of a UV-based micro-syringe deposition ( $\mu$ SD) system utilized in the micro-dispensing of photopolymers on particulate ceramic substrates. This methodology is used in embedding functionally graded and interconnected micro-features within constructs produced by a novel combined powder-based additive manufacturing (AM) and UV-based micro-syringe deposition $(\mu \mathrm{SD})$ technique. The process is experimentally characterized using SEM and optical microscopy to study the effect of a wide range of process parameters on the geometrical quality of deposited tracks. Experimental data show that the system can produce features ranging from 200 to $575 \mu \mathrm{m}$ in width and from 20 to $200 \mu \mathrm{m}$ in height on particulate ceramic surfaces. To gain insight into the proposed micro-deposition process, a two-tier model is also developed. The first framework describes an analytical model for predicting the flow rate of the dispensed photopolymer fluid based on the piston displacement. The second model is a stochastic framework for predicting the line width of the features deposited on the substrate using a Monte Carlo probabilistic simulation to compensate for uncertainty in the system input parameters. A comparison between experimental and modeling line width predictions shows that the modeling results are $14-38 \%$ higher than the experimental results, depending on the system input variables. The proposed model is enhanced by introducing adjustment factors to compensate for UV exposure delay, fluid migration, and imbibition.
\end{abstract}

(c) 2013 Elsevier B.V. All rights reserved.

\section{Introduction}

Powder-based additive manufacturing (AM) is a highly versatile layer-by-layer fabrication process. Using this manufacturing approach, it is possible to build very complex three-dimensional (3D) computer-aided design (CAD) models based on the required internal and external architecture of the part while considering the constraints imposed by system capabilities. This process suffers from inherent limitations in terms of the smallest achievable internal features. The smallest internal feature is influenced by the binder injection parameters, binder viscosity, lateral binder infiltration and liquid imbibition as concluded in the review on indirect three-dimensional printing by Lee et al. (2005). In a review on rapid prototyping techniques, Yang et al. (2002) concluded that in addition to binder dispensing factors, the powder particle size and powder compaction also contribute to the high potential for having trapped particles inside the part when designing internal

\footnotetext{
* Corresponding author. Tel.: +1 519888 4567x37560; fax: +1 5198884333

E-mail addresses: ehsan.toyserkani@uwaterloo.ca, etoyserk@uwaterloo.ca (E. Toyserkani).
}

cavities, channels or macro-pores. The review work by Leong et al. (2003) also supports the idea that the feature size is one important limitation using powder-based AM approaches. In more recent assessments of powder-based additive manufacturing via threedimensional printing, Butscher et al. (2011) as well as the review done by Castilho et al. (2011) outline that this method is highly valuable due to the high versatility of materials and complex part geometry which can be manufactured; however the limitations in having support particles trapped within the internal features of the part and resolution limitations remain a topic of study. The above-mentioned review works on powder-based additive manufacturing techniques, all reach the same consensus that this type of technology is highly versatile but it is still very difficult to achieve internal interconnected or isolated features below $500 \mu \mathrm{m}$ in size using this methodology. This issue becomes even more pressing in manufacturing constructs with complex conformal channels, as it becomes increasingly difficult to remove trapped support materials. This serious limitation currently hinders the feasibility of producing parts with appropriate conformal channels required in various industrial and bio-medical applications.

One such application of powder-based AM is in fabricating bioceramic scaffolds for bone and osteochondral tissue engineering, 


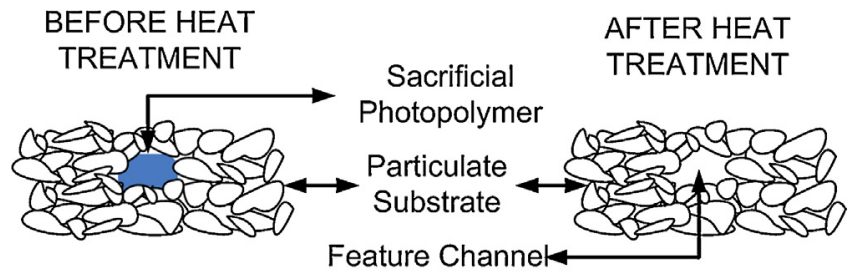

a)

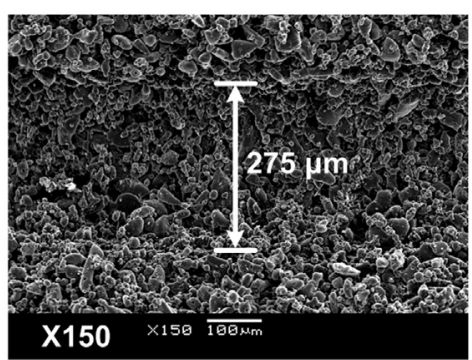

b)

Fig. 1. (a) Combined additive manufacturing and micro-syringe deposition (AM-SD) process for creating structures with interconnected features. (b) An example of such a channel obtained after the heat treatment protocol, viewed under SEM $(150 \times)$.

where the desired range of interconnected micro-sized channels is dictated by the tissue section being replaced or enhanced as postulated by Hutmacher (2000). In the case of tissue engineered scaffolds for bone and cartilage augmentation or replacement, the internal structural architecture of the scaffolds is crucial in promoting tissue ingrowth and vascularization (Butscher et al., 2011). Yang et al. (2001) undertook an extensive review of appropriate geometrical factors for such scaffolds and concluded that interconnected porosities in a range $100-350 \mu \mathrm{m}$ are appropriate for bone regeneration and furthermore, fibro-vascularization is promoted by interconnected porosities in the range of $500 \mu \mathrm{m}$. Chang et al. (2000) in their study on osteoinduction in porous hydroxyapatite conclude that the desired range of interconnected pore or channel size for tissue engineered bone and cartilage scaffolds varies between 100 and $500 \mu \mathrm{m}$. This conclusion is also validated by Kujala et al. (2003) while reporting on osteointegration into nickel-titanium implants.

Various research teams have attempted to modulate the appropriate features and mechanical properties for constructing bioceramic scaffolds with embedded interconnected features for bone and osteochondral tissue replacement or augmentation. Woesz et al. (2005) used an indirect AM technique to produce a resin mold that can be immersed in the bioceramic slurry. The mold was produced using a photo-sensitive liquid via stereolithography. During sintering, the mold disintegrates, leaving behind the designed features in the range 300-500 $\mu \mathrm{m}$. An indirect approach was also used by Lee et al. (2005) in fabricating poly(D,L-lactic-Coglycolic acid) (PLGA) scaffolds by making use of a plaster mold and sucrose porogens. They succeeded in making channels with a feature diameter averaging $700 \mu \mathrm{m}$. The above-mentioned techniques are indirect, requiring multiple manufacturing steps and involving a range of solvents. The most important shortcoming of these methods is that they are often not adept in having precise control over internal feature characteristics of the scaffold. (Warnke et al., 2010) used three-dimensional printing to test the manufacturability of scaffolds with features in a range $>500 \mu \mathrm{m}$ and the biocompatibility of hydroxyapatite and tricalcium phosphate materials. The range of features achieved using this powder-based additive manufacturing method is limiting, as it is at the high spectrum required for bone and osteochondral tissue augmentation.

To address the abovementioned limitations, we initiated a study to enhance control of internal dimensional features of ceramic parts produced using powder-based additive manufacturing. To this end, a combined additive manufacturing and micro-syringe deposition (AM- $\mu \mathrm{SD})$ technology is proposed where a dispensing system capable of depositing micro-sized sacrificial polymeric networks on particulate surfaces throughout the construct is incorporated into the system. Using this process, a controlled feature size of a photopolymer in the target range $100-500 \mu \mathrm{m}$ is deposited on particulate ceramic surfaces. The photopolymer is then burned off in a furnace during sintering to produce interconnected channels. This approach allows for control of internal features by avoiding loose support powder material from being trapped inside largersized parts.

In this article, the polymer deposition on a bioceramic powder substrate is studied experimentally and numerically based on a two-tier model developed for characterizing the fluid flow out of the $\mu$ SD nozzle as well as the final geometry of the tracks deposited on the powder layer. Experimental data are used to initialize and validate the model and also establish a rich characterization of this complex process.

\section{Brief description of the combined AM- $\mu$ SD system}

The purpose of the micro-syringe deposition ( $\mu \mathrm{SD}$ ) system is to deposit sacrificial polymers throughout the part to produce a network of tracks which mimic the complexity of required internal interconnected channels. The deposited structures will be disintegrated during post-heat treatment processing, leaving behind

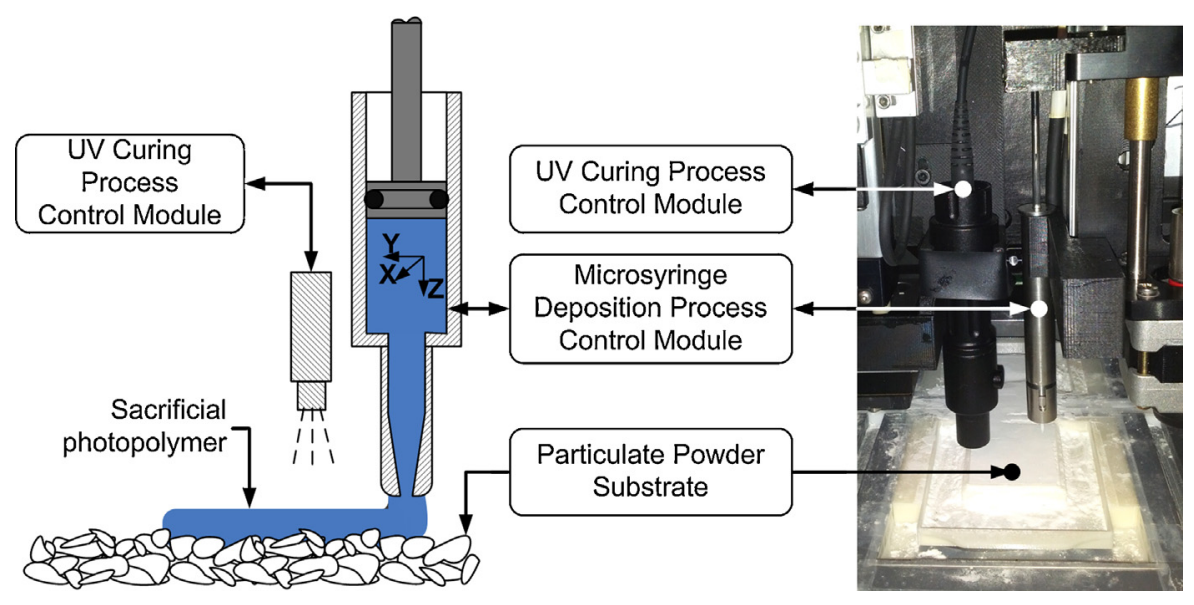

Fig. 2. Relevant hardware modules as a part of the AM- $\mu$ SD system. 
the corresponding macro-porous channels and cavities as seen in Fig. 1. The first step in the process is the development of a 3D CAD model based on the required internal and external architecture while considering manufacturability and constraints. The CAD model is then dissected into thin 2D image slices which can be manufactured using the powder-based AM- $\mu$ SD system in a layerby-layer fashion. Within each layer, the powder is first spread using a counter-rotating roller mechanism and then selectively bonded together at specific locations by injecting a binder using an inkjetlike printing technology. A new layer of powder is subsequently spread on top of the current layer. The cycle is repeated until the part is completed. On specific layers, a sacrificial photopolymer is injected to create the required voids and channels. The green part is exposed to a specific heating protocol to first remove the sacrificial polymer and then create agglomerated (sintered) particles. Further information about the AM- $\mu$ SD process and materials used in it can be found in our recent article (Vlasea et al., 2013).

For the polymer deposition process, the relevant hardware modules are illustrated in Fig. 2. The $\mu$ SD process control module is comprised of a modified modular piston-driven pump apparatus (70-2225 UL Modular Pump, Harvard Apparatus, Quebec, Canada) mounted on a high precision $x y z$ positioning system. The $\mu S D$ Process Control Module is used to deposit the photopolymer track on the powder substrate. The $\mu$ SD system uses a ceramic deposition nozzle (1573-xx-437GM, CoorsTek, Inc. Gaiser Products Group, Ventura, CA, USA) adapted for this application. The custom ceramic nozzle minimizes droplet accumulation at the tip, does not chemically interact with the material, it preserves its dimensional properties under elevated temperatures and is reliable for multiple injection cycles. The UV curing process module is a solid-state UV spot light (SUV-01, Algitron, Woburn, MA, USA), $385 \mathrm{~nm}$ wavelength, $2.0-3.2 \mathrm{~W} / \mathrm{cm}^{2}$ intensity, $4 \mathrm{~mm}$ spot size at a distance of $20 \mathrm{~mm}$ used to cure the photopolymer track extruded through the $\mu \mathrm{SD}$ system. There is a physical offset of $5 \mathrm{~cm}$ in the $y$-direction between the $\mu$ SD nozzle and the UV curing head to avoid photopolymerization of fluid inside the nozzle.

\section{Analytical model development}

The deposited polymer feature size has a direct impact on the resulting porosity and interconnected channel geometry within the part. It is therefore important to gain insight into the $\mu$ SD system behavior and predict the final feature size by developing a model. In this study, a two-level model is developed to link the fluid deposition parameters with the final geometry of the fluid deposited on the powder substrate.

\subsection{Pressure-flow model}

An approximate analytical model is used to estimate the flow of a fluid through the nozzle geometry based on the micro-syringe piston displacement. For the dispensing system considered in this study, we assume that the process is isothermal, fluid properties are time-independent and the flow is fully developed laminar throughout the dispensing barrel and nozzle. The model presented by Chen and Kai (2004) in their work on modeling of positive-displacement fluid dispensing processes, is easy to adapt to this particular system and it is also relatively straightforward to implement in a programming environment. The model described by Chen and Kai (2004) assumes that non-Newtonian effects are neglected if the dispensing barrel is large enough, minor losses are not significant at low velocities, the static pressure inside the syringe is assumed to be uniform since the height of the fluid in the syringe is relatively small, the process is isothermal and the fluid is compressible. Considering the above assumptions, Eq. (1) describes a general hydro-dynamic
Table 1

Geometrical characteristics of dispensing system.

\begin{tabular}{lll}
\hline Description & Parameter & Value \\
\hline Piston diameter & $D_{p}$ & $3.36 \mathrm{~mm}$ \\
Nozzle length & $L_{n}$ & $15 \mathrm{~mm}$ \\
Nozzle exit diameter & $D_{n}$ & $250 \mu \mathrm{m}$ \\
\hline
\end{tabular}

physics predicating the pressure build-up inside the syringe barrel due to the motion of piston as used in the model development by Chen and Kai (2004).

$A_{p} \dot{Z}-Q=\left[\frac{V_{o}-A_{p} Z}{\beta}\right] \dot{P}$

where $V_{o}$ is the initial volume of fluid in the dispenser, $A_{p}$ is the area of the piston, $Z$ is the piston vertical displacement as shown in Fig. 2, $\beta$ is the fluid effective bulk modulus under the assumption that there is no gas trapped in the fluid compartment, $Q$ is the flow out of the nozzle and $P$ is the pressure built-up inside the dispenser.

The Laplace function presented in Eq. (2) was adapted by Chen and Kai (2004) and it describes the fluid flow rate leaving the nozzle driven by the pressure build-up inside the syringe barrel. It applies under the assumption that the fluid flow is laminar, timeindependent, there is a no slip condition at the nozzle wall, and that there are minimal compressibility effects inside the nozzle.

$\frac{Q(s)}{P(s)}=\frac{Q_{u}}{s \rho L_{n} Q_{u} /\left(\pi D_{n}^{2} / 4\right)+1}$

where $Q_{u}$ is the steady state fluid flow under a unit of pressure calculated using the generalized power law as described in the mathematical model by Chen et al. (2000), $\rho$ is the fluid density, $L_{n}$ is length of the nozzle and $D_{n}$ is the nozzle internal diameter. The fluid properties are described in Section 4.2.

Eqs. (1) and (2) have been used to approximate the pressure build-up and flow rate of fluid through the $\mu$ SD system based on the piston displacement. The outputs from this model are used as inputs in developing the secondary deposition geometry model. Table 1 lists the dispensing system parameters as used in this modeling framework.

\subsection{Deposition geometry model}

The deposition geometry model is used to predict the crosssectional geometry of the deposited track. The inputs to this model are the pressure and flow parameters developed in the pressure-flow model as well as the velocity of the substrate, nozzle geometrical characteristics and fluid properties. To account for liquid imbibition into the powder substrate, the effect of UV exposure latency has been quantified experimentally and can be used as a compensation factor in the modeling scheme. The proposed model has been adapted from the work done by Vozzi et al. (2002) on characterizing geometrical features of extrusion-based polymer scaffolds and is used to approximate the width of the deposited patterns based on fluid conditions at the nozzle. In this model, it is assumed that the fluid pressure is the main driving factor and that the dispensed polymer is a viscous Newtonian fluid. Additionally, the model assumes that the final cross-sectional profile of the dispensed feature can be approximated to be an elliptical segment as describe by Vozzi et al. (2002). Eq. (3) describes the relationship between the line width and the applied fluid pressure, velocity of the substrate, empirically measured feature height and polymer viscosity.

$a=\frac{\pi R D_{n} P}{16 \mu v_{0} h h_{z}}$

where $a$ is the resulting line width, $h$ is an empirical approximation of the height of the polymer pattern, $v_{0}$ is the velocity of the 


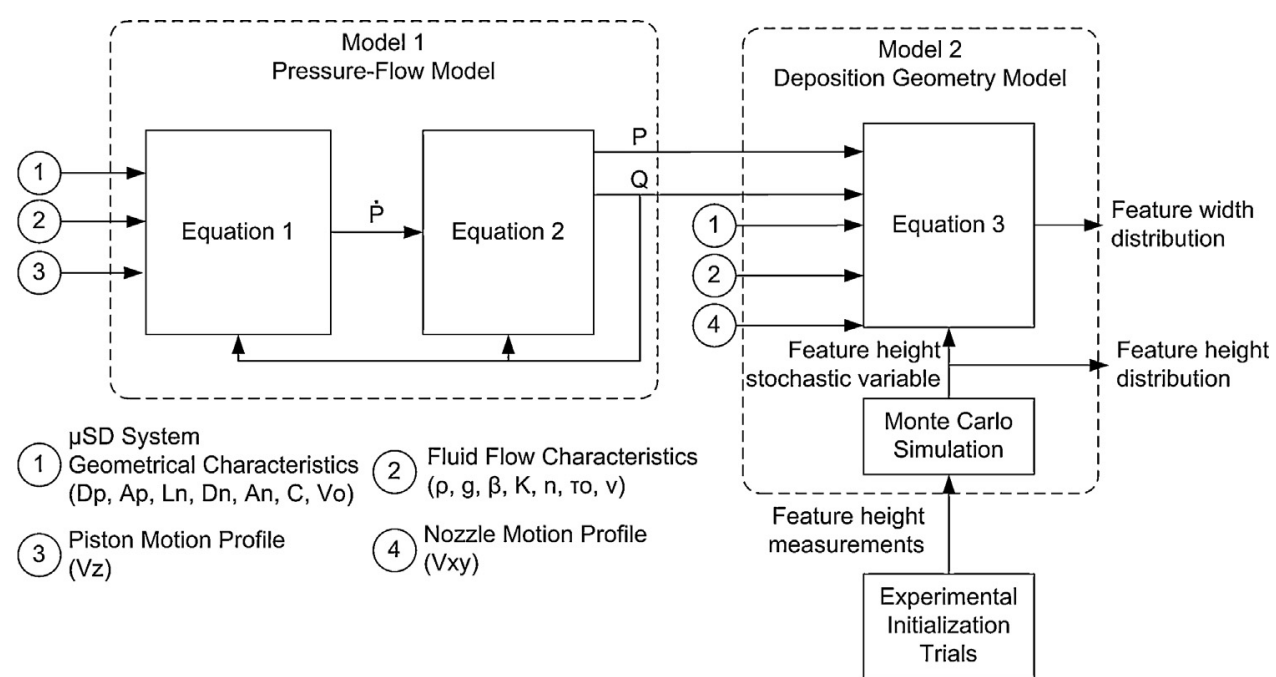

Fig. 3. Block diagram presentation of the combined analytical "pressure-flow" model and stochastic "deposition geometry" model.

substrate with respect to the nozzle, $D_{n}$ is the internal radius of the tip of needle, $\mu$ is the viscosity of the polymer, and $h_{z}$ is the length of the tapered zone of the capillary, which is estimated at $1 \mathrm{~mm}$. This model assumes knowledge of the line height, which would ideally be a feedback measurement from a vision system. In this paper, to test the feasibility of such a feedback scheme, the line height for each set of operation conditions was considered to be a stochastic variable with a probability distribution obtained through empirical data measured during an initialization phase where height measurements are taken at each desired combination of input parameters. The impact of this stochastic variable on the model output is studied by running a Monte Carlo simulation with 100 independent trials to determine the sensitivity of the model to height parameter. The modeling results are then compared with experimental measurements to quantify the performance of the model. The combined model is illustrated in Fig. 3.

\section{Materials and methods}

\subsection{Structure material}

A type of ceramic called calcium polyphosphate (CPP) is amongst one of the more promising materials for producing structures for bone replacement. In this study, calcium polyphosphate (CPP) powder with particle sizes of $75-100 \mu \mathrm{m}$ was used as the structure material along with polyvinyl alcohol (PVA) $5 \mathrm{wt} \%$ solution in de-ionized water (DI) as a binder. The binder was delivered by a piezo-based print-head (XAAR, 1001 model, Xaar, Cambridge, UK).

\subsection{Sacrificial photopolymer}

The photopolymer solution used in this paper is comprised of a monomer, a reactive dilutant mixed to achieve the desired injectability and a photoinitiator (PI) to control the UV light sensitivity. Ethoxylated (10 bisphenolAdiacrylate) (EBA) (Ebecryl 150, Cytec, NJ, USA) was used as a monomer. Cellulose acetate butyrate (CAB) (Sigma Aldrich, Oakville, Canada) was dissolved in acetone and functions as a dilutant. $C A B$ is also used to control the viscosity of the final photopolymer solution. Phenylbis $(2,4,6-$ trimethylbenzoyl) phosphine oxide (Irgacure 819) (Sigma Aldrich, Oakville, Canada) was utilized as a PI. The components were utilized as received and mixed in solution at room temperature with a composition of $13 \mathrm{wt} \% \mathrm{CAB}, 56 \mathrm{wt} \% \mathrm{EBA}, 30.5 \mathrm{wt} \%$ acetone, and $0.5 \% \mathrm{PI}$.

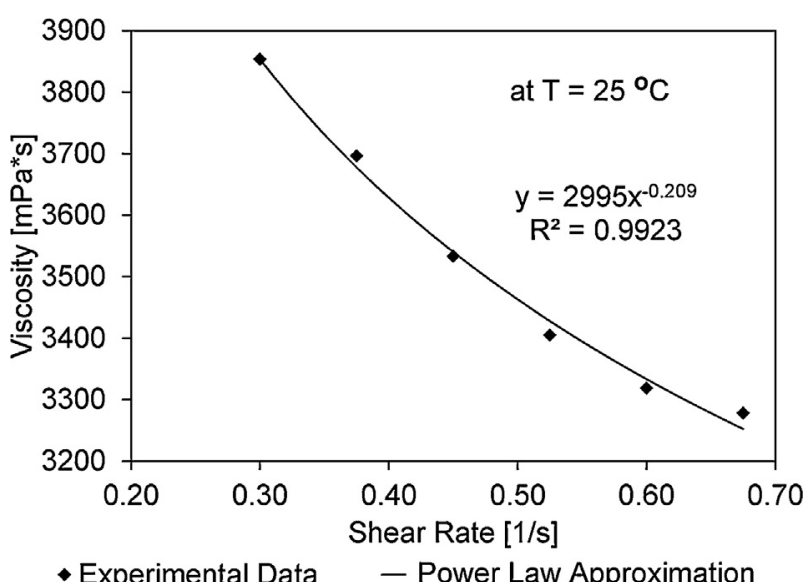

Fig. 4. Photopolymer fluid power law approximation.

A PI concentration of $0.5 \mathrm{wt} \%$ resulted in an acceptable photopolymerization time of approximately $1 \mathrm{~s}$ when exposed to a UV light (Solid State UV Spot Light, Algitron, Woburn, MA, USA) radiated from a distance of $10 \mathrm{~mm}$. The solution is dispensed at room temperature. The flow behavior of the photopolymer was characterized using a rheometer (DW-III Ultra, Brookfield Engineering Laboratories, Middleboro, MA, USA) at room temperature. The shear stress and shear rate data were curve-fitted using the generalized fluid power law to determine the viscosity $\mu$, consistency index $K$ and flow behavior index $n$ as seen in Fig. 4 .

Table 2 summarizes the fluid flow properties computed based on the power law approximation summarized in Fig. 4. The viscosity $\mu$ was computed as the average over the shear domain. The consistency index $K$ and flow behavior index $n$ were determined based on the approximated power law arguments, where $K$ is the constant and $\mathrm{n}$ is the difference between unity and the approximated exponent argument.

Table 2

Photopolymer fluid flow properties.

\begin{tabular}{lll}
\hline Description & Parameter & Value \\
\hline Viscosity & $\mu$ & $3334 \mathrm{mPas}$ \\
Consistency index & $K$ & $2995 \mathrm{~Pa}$ \\
Flow behavior index & $n$ & 0.791 \\
\hline
\end{tabular}



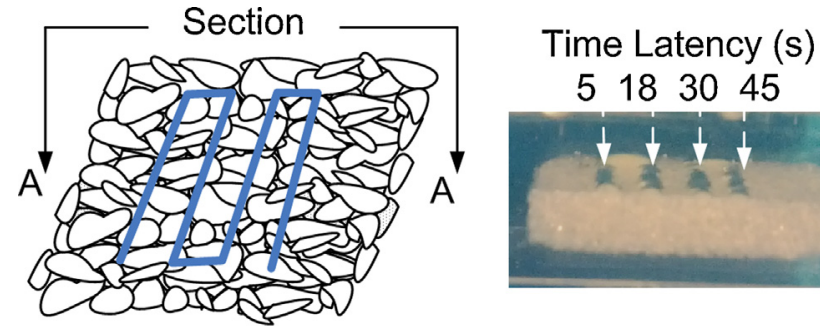

5183045
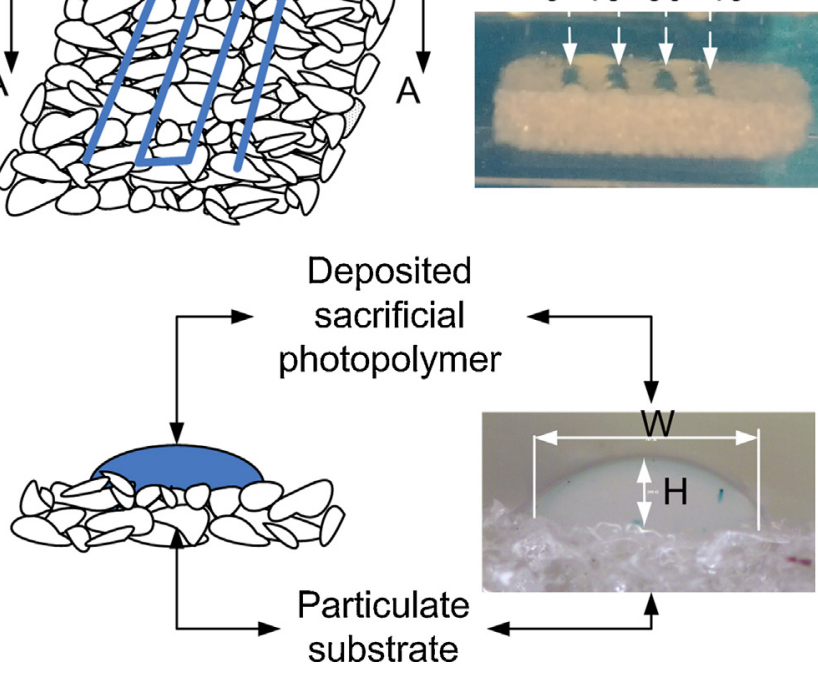

Fig. 5. Fabricated sample after slicing and polishing (encapsulated in epoxy).

\subsection{Sample fabrication and preparation}

The selected variables of interest for this study in experimental and simulation trials are the $\mu$ SD piston linear velocity $\dot{Z}$ and the velocity of the nozzle with respect to the substrate $V_{x y}$. The piston linear velocity $\dot{Z}$ is directly proportional to the steady state fluid flow $Q$ leaving the nozzle; therefore parameter $\dot{Z}$ can be represented by the corresponding flow $Q$. The selected parameter values for $Q$ and $V_{x y}$ are shown in Table 3 . These values were chosen based on a pre-screening experimental feasibility study. The deposition was performed on $10 \times 25 \mathrm{~mm}^{2}$ and $2 \mathrm{~mm}$ thick particulate ceramic prefabricated substrates. The nozzle deposition height was maintained at a distance of approximately one nozzle inner diameter of $250 \mu \mathrm{m}$ as per recommendation found in the polymer deposition process described by Vozzi et al. (2002).

In this work, 16 samples were fabricated for each of the model initialization and model validation phases. Using the AM- $\mu S D$ approach, the samples were manufactured by first printing rectangular pieces $10 \times 25 \mathrm{~mm}^{2}$ and $2 \mathrm{~mm}$ thick and subsequently depositing $20 \mathrm{~mm}$ long photopolymer tracks in a straight line on the CPP surface. On each sample, four tracks were deposited, each track with a different UV exposure latency $T$. The parts were then encapsulated in an epoxy mix. Height and width measurements were taken by slicing the parts orthogonally at $n=6$ locations, polishing and measuring as observed in Fig. 5.

\subsection{Geometrical property measurements}

All samples were encapsulated in epoxy and polished. The geometrical properties, height and width, of features were consistently analyzed using a calibrated camera (EDMUND Optics Inc., NJ, USA) at $2.3 \times$ magnification with a calibration factor of 2.7397 .

\section{Results}

\subsection{Experimental measurements versus simulated outputs}

Experimental data show that tracks with width ranging from 200 to $575 \mu \mathrm{m}$ and height from 20 to $200 \mu \mathrm{m}$ are feasible, depending on system input variables. The measured widths from experimental trials were compared with the corresponding predictions from the developed model to investigate the model
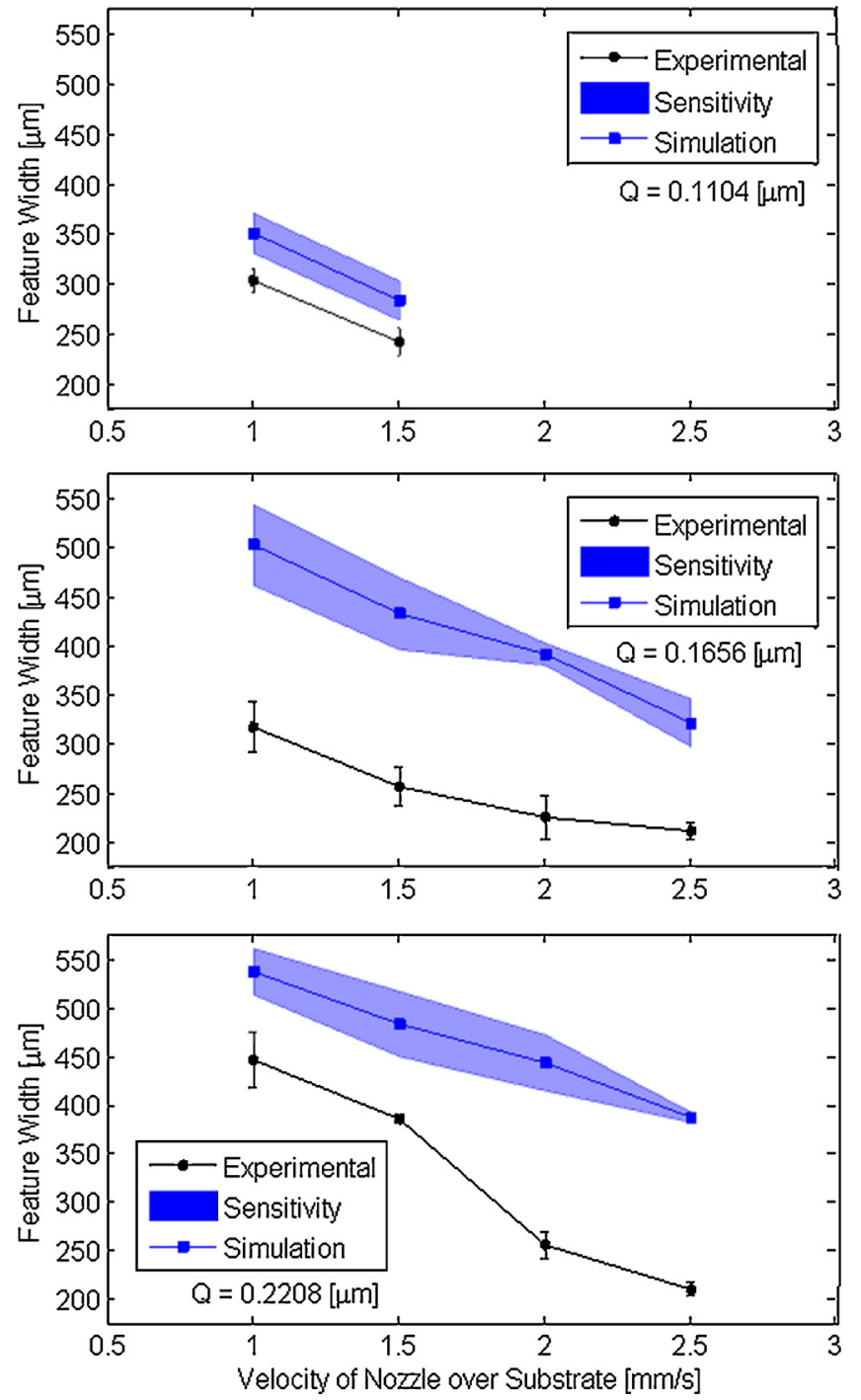

Fig. 6. Graphs illustrate the dependence of track width with respect to the velocity of the nozzle in the $x y$ direction at different volumetric flow throughputs. The values indicate experimental vs. modeled data with corresponding sensitivity domain based on Monte Carlo simulations.

effectiveness. All comparisons between experimental and simulated data are for the $5 \mathrm{~s}$ UV latency exposure condition. Fig. 6 summarizes the experimental and corresponding simulated results at different fluid flow rates, under varying nozzle head velocities. The results show that the simulated outputs were considerably higher than the experimental measurements. The simulated outputs in Fig. 6 include a Monte Carlo sensitivity band illustrating the model sensitivity with respect to the stochastic line height measurement obtained in the initialization trial. The model results were an average of $14 \%$ higher for a fluid flow of $0.1104 \mu \mathrm{L} / \mathrm{s}, 38 \%$ for a fluid flow of $0.1656 \mu \mathrm{L} / \mathrm{s}$, and $30 \%$ for a fluid flow of $0.2208 \mu \mathrm{L} / \mathrm{s}$ as seen in Fig. 6. These values can be designated as adjustment factors, where the model simulation results are compensated based on the deviation between simulated and experimental results at each flow condition respectively.

\subsection{Effect of UV exposure latency on experimental measurements}

Four latency times were implemented: $5 \mathrm{~s}, 18 \mathrm{~s}, 30 \mathrm{~s}$, and $45 \mathrm{~s}$. Of the four latency times, the $45 \mathrm{~s}$ latency did not produce measurable or reliable samples, as the fluid infiltrated the porous substrate almost entirely. The results in Fig. 7 show that for the experiments 
Table 3

Selected variables in this study.

\begin{tabular}{|c|c|c|c|c|c|}
\hline Trial & $\begin{array}{l}\text { Volumetric } \\
\text { flow } Q(\mu \mathrm{L} / \mathrm{s})\end{array}$ & $\begin{array}{l}\text { Substrate velocity } \\
V_{x, y}(\mathrm{~mm} / \mathrm{s})\end{array}$ & $\begin{array}{l}\text { Model initialization } \\
n \text { (samples) }\end{array}$ & $\begin{array}{l}\text { Model validation } n \\
\text { (samples) }\end{array}$ & Empirical observations \\
\hline 1 & 0.1104 & 1.0 & 6 & 6 & Successful \\
\hline 2 & & 1.5 & 6 & 6 & Successful \\
\hline 3 & & 2.0 & 6 & 6 & a \\
\hline 4 & & 2.5 & 6 & 6 & a \\
\hline 5 & 0.1656 & 1.0 & 6 & 6 & Successful \\
\hline 6 & & 1.5 & 6 & 6 & Successful \\
\hline 7 & & 2.0 & 6 & 6 & Successful \\
\hline 8 & & 2.5 & 6 & 6 & Successful \\
\hline 9 & 0.2208 & 1.0 & 6 & 6 & Successful \\
\hline 10 & & 1.5 & 6 & 6 & Successful \\
\hline 11 & & 2.0 & 6 & 6 & Successful \\
\hline 12 & & 2.5 & 6 & 6 & Successful \\
\hline 13 & 0.2761 & 1.0 & 6 & 6 & a \\
\hline 14 & & 1.5 & 6 & 6 & a \\
\hline 15 & & 2.0 & 6 & 6 & a \\
\hline 16 & & 2.5 & 6 & 6 & $a$ \\
\hline
\end{tabular}

a Inconsistent deposition, tracks were intermittent. Measurements could not be taken.
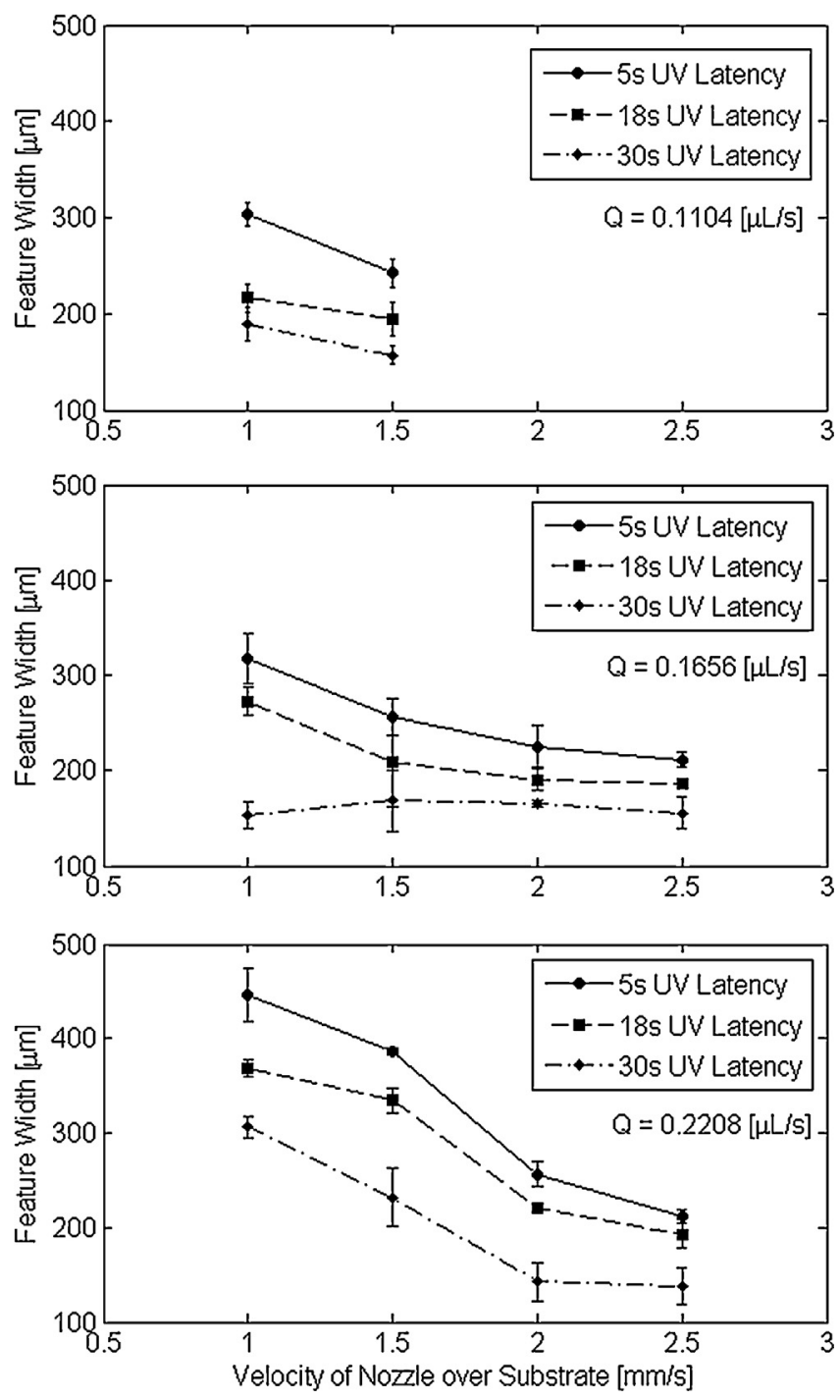

Fig. 7. Graphs illustrate the effect of UV exposure latency of $5 \mathrm{~s}, 18 \mathrm{~s}$ and $30 \mathrm{~s}$ on the experimental measured line width. An increase in UV exposure latency decreases the observed line width.

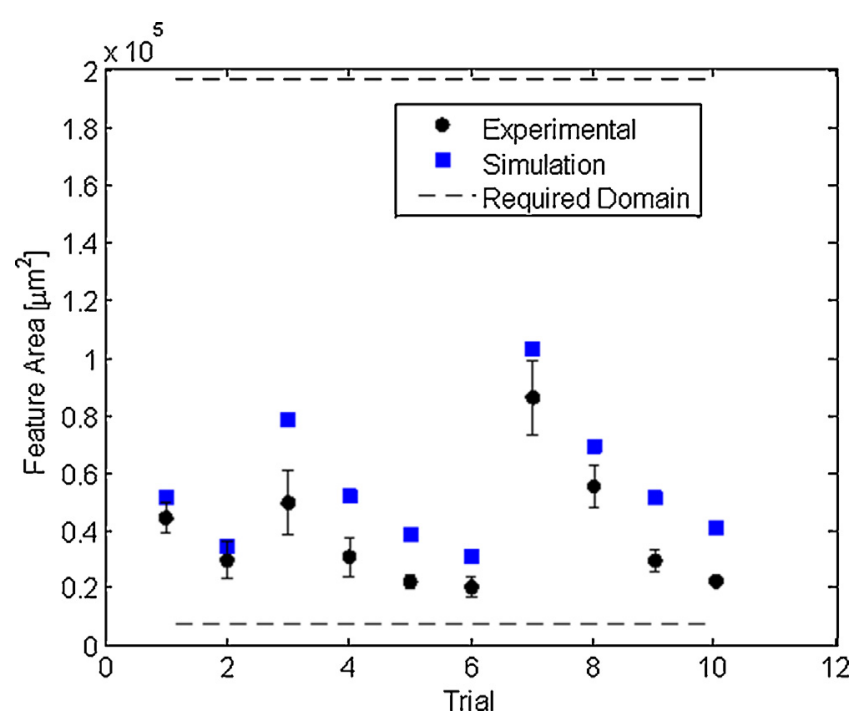

Fig. 8. Cross-sectional area of experimental and simulated features versus the minimum and maximum threshold of cross-sectional areas corresponding to features in the range $100-500 \mu \mathrm{m}$ in diameter respectively.

conducted at low, moderate and high flow conditions, a latency of $18 \mathrm{~s}$ decreases line width by an average of $17 \%$, while a latency of 30 s decreases the line width by an average of $36 \%$ when compared with the line width measured at time latency of $5 \mathrm{~s}$ respectively.

\subsection{Desired feature range vs. experimental and simulated results}

A central purpose of this work was to test the capability of the AM- $\mu$ SD system in producing features within a desired range. The desired feature range selected for this study is applicable for osteochondral scaffold manufacturing and lays between $100 \mu \mathrm{m}$ and $500 \mu \mathrm{m}$ in diameter. Fig. 8 illustrates the cross-sectional area of experimental and compensated simulated features versus the minimum and maximum threshold of cross-sectional areas corresponding to the desired features in the range $100-500 \mu \mathrm{m}$ in diameter respectively.

\section{Discussion}

In this work, the focus was to characterize the geometrical features of polymeric tracks deposited onto porous substrates using 
a combined additive manufacturing and micro-syringe deposition (AM- $\mu \mathrm{SD}$ ) novel approach. This method is superior to the indirect approaches currently used in this field due to its simplicity, as it does not require extensive manufacturing steps such as developing a mold or a mask. In addition, it establishes a platform to incorporate micro-scale interconnected channels inside ceramic parts that is not possible with conventional additive manufacturing methods. To this effect, experimental trials were performed to study the effect of operational input parameters such as velocity of nozzle with respect to the substrate, volumetric flow throughput, and ultra violet exposure latency on the geometrical characteristics of the deposited photopolymer onto a particulate substrate. An analytical model was adapted based on the existing work to predict the process performance in terms of characterizing fluid flow and geometrical properties of the deposited feature. The experimental results were used to validate the model performance.

Experimental data show that the $\mu$ SD process was successfully capable of depositing features with a cross-sectional width between 200 and $575 \mu \mathrm{m}$ and the height between 20 and $200 \mu \mathrm{m}$ as seen in Fig. 8, which are within the desired range for various applications such as manufacturing of osteochondral scaffolds for bone and cartilage augmentation. Both the experimental and simulated results fall within the lower spectrum of the desired feature range. It is hypothesized that the feature size can be further increased by increasing the inner diameter of the nozzle in accordance with the deposition geometry model used in this study. Furthermore, as seen in Fig. 6, the width of tracks decreases when the process speed increases from 1 to $2.5 \mathrm{~mm} / \mathrm{s}$. Within this range and considering the standard deviations, the correlation of these two parameters is relatively linear and can be used to further control the desired feature width.

The experimental data were compared to outputs from the proposed model. The set of simulated and experimental results are summarized in Fig. 6 . The two sets of data show similar trends, with simulated data showing consistently higher values than the experimental results. The lower experimental results can be attributed to liquid imbibition into the porous substrate influenced by the fluid contact angle and surface tension, powder particle size and substrate pore size, fluid saturation levels as well as the time latency between deposition and UV exposure.

The UV exposure latency is a considerable factor that affects the feature shape integrity after deposition as seen in Fig. 7. Ideally, the photopolymer should be cured as it is being extruded through the nozzle in order to preserve the integrity of the deposited track. To avoid clogging, the UV spot light has a physical offset from the nozzle which requires a latency to re-align the deposition substrate with the UV spot light. Based on these experimental results, it can be concluded that the feature width can also be controlled by adjusting the latency, as a latency of $18 \mathrm{~s}$ decreases line width by an average of $17 \%$, while a latency of $30 \mathrm{~s}$ decreases the line width by an average of $36 \%$ when compared with the line width measured at time latency of $5 \mathrm{~s}$, respectively.

Fluid migration and imbibition through the porous powder substrate are other factors that affect the shape integrity of the deposited feature. Fluid migration through porous powder media is influenced by the contact angle between fluid and substrate, liquid surface tension, powder particle size, and pore size as discussed by Lee et al. (2005) in their study on the feasibility of constructing parts with interconnected channels using powder-based AM. Improved feature integrity can be achieved by increasing the viscosity of the fluid to reduce the rate of fluid imbibition, resulting in better shape preservation after fluid extrusion. This conclusion is supported by the theory developed by Batten (1984) in his theoretical work on liquid imbibition in porous substrates. Based on these insights, it can be concluded that in this process, the feature width can also be controlled by changing the viscosity of the photopolymer. In this work, viscosity is proportional with the \%wt of cellulose acetate butyrate (CAB) in solution.

To better predict experimental results, the developed model can be enhanced by using the compensation factors as identified in Section 5.1 to account for fluid imbibition and UV exposure latency. Based on experimental and simulated data, the compensation factors $14 \%, 38 \%$ and $30 \%$ corresponding to the $0.1104,0.1656$, and $0.2208 \mu \mathrm{L} / \mathrm{s}$ flow rate, are not proportional with the fluid flow. This may indicate that the rate of fluid imbibition may be influenced by fluid saturation levels in the powder substrate. The phenomenon of local fluid saturation is complex, especially in heterogeneous porosities and is influenced by the primary and secondary capillary flow as described by Markicevic et al. (2012). They have shown that liquid saturation in the direction of principal flow through a porous medium can have a complex profile depending on pore boundary conditions and interfacial forces. Based on these observations, the model proposed by Markicevic et al. (2012) can be adapted and included in the present AM- $\mu$ SD model to predict the interaction between the powder substrate and photopolymer in the time span between deposition and photopolymerization. Using this approach, the compensation factors can be dynamically computed during runtime, rendering the model more relevant for deposition on a porous substrate.

\section{Conclusions}

The proposed AM- $\mu$ SD methodology for depositing sacrificial substrates onto porous particulate substrates is a promising and novel approach for embedding interconnected macro-channels within parts produced via powder-based additive manufacturing. Using the AM- $\mu S D$ process, the internal feature characteristic can be controlled via process input parameters such as fluid flow, deposition nozzle speed, nozzle diameter, and UV exposure latency. It was shown that by using a $250 \mu \mathrm{m}$ nozzle for a flow range between 0.1104 and $2208 \mu \mathrm{L} / \mathrm{s}$ and a nozzle velocity with respect to the substrate between 1 and $2.5 \mathrm{~mm} / \mathrm{s}$, with a UV exposure latency of $5 \mathrm{~s}$, the resulting features range in width between 200 and $575 \mu \mathrm{m}$ and in height between 20 and $200 \mu \mathrm{m}$ on particulate substrates. The deposition process has been characterized experimentally and in simulation. The simulated results are $14-38 \%$ higher than experimental results over the input parameter range. The results suggest that for a specific diameter of a deposition nozzle, the feature size can be controlled by changing the fluid flow, the process speed, the UV exposure latency, and the fluid viscosity. These factors are shown to affect the geometrical integrity of the sacrificial polymer post-injection and can be used as process control parameters. Furthermore, the developed model can be used in an online control paradigm if compensation factors are included to account for the effects of depositing on a porous substrate. To conclude, the feasibility of using a novel AM- $\mu$ SD approach was validated experimentally. The complexity of controlling the $\mu \mathrm{SD}$ process is amplified by the interaction of the sacrificial fluid with the porous substrate. To better understand and control the process, the $\mu$ SD system was modeled to quantify the effects of each input parameter on the final geometrical characteristic of the feature and to devise strategies to improve the performance of the proposed model.

\section{Acknowledgments}

The authors appreciate the funding support of the Department of Defense US Army (Medical Research Acquisition Activity - Grant number of OR090169) as well as the Ontario Graduate Scholarship awarded to the first author. 


\section{References}

Batten, G.L., 1984. Liquid imbibition in capillaries and packed beds. Journal of Colloid and Interface Science 102 (2), 513-518, http://dx.doi.org/10.1016/ 0021-9797(84)90254-6.

Butscher, A., Bohner, M., Hofmann, S., Gauckler, L., Müller, R., 2011. Structural and material approaches to bone tissue engineering in powder-based threedimensional printing. Acta Biomaterialia 7 (3), 907-920, http://dx.doi.org/10. 1016/j.actbio.2010.09.039, Acta Materialia Inc.

Castilho, M., Pires, I., Gouveia, B., Rodrigues, J., 2011. Structural evaluation of scaffolds prototypes produced by three-dimensional printing. International Journal of Advanced Manufacturing Technology 56 (5-8), 561-569, http://dx.doi. org/10.1007/s00170-011-3219-4.

Chang, B.S., Lee, C.K., Hong, K.S., Youn, H.J., Ryu, H.S., Chung, S.S., Park, K.W., 2000. Osteoconduction at porous hydroxyapatite with various pore configurations. Biomaterials 21 (12), 1291-1298, Retrieved from http://www.ncbi.nlm.nih. gov/pubmed/19845154

Chen, X.B., Kai, J., 2004. Modeling of positive-displacement fluid dispensing processes. IEEE Transactions on Electronics Packaging Manufacturing 27 (3), 157-163, http://dx.doi.org/10.1109/TEPM.2004.843083.

Chen, X.B., Schoenau, G., Zhang, W.J., 2000. Modelling of time-pressure fluid dispensing processes. IEEE Transactions on Electronics Packaging Manufacturing 23 (4), 300-305, http://dx.doi.org/10.1109/TEPM.2000.895060.

Hutmacher, D.W., 2000. Scaffolds in tissue engineering bone and cartilage. Biomaterials 21 (24), 2529-2543, Retrieved from http://www.ncbi.nlm. nih.gov/pubmed/11071603

Kujala, S., Ryhanen, J., Danilov, A., Tuukkanen, J., 2003. Effect of porosity on the osteointegration and bone ingrowth of a weight-bearing nickel-titanium bone graft substitute. Biomaterials 24 (25), 4691-4697, http://dx.doi.org/10.1016/ S0142-9612(03)00359-4.

Lee, M., Dunn, J.C.Y., Wu, B.M., 2005. Scaffold fabrication by indirect threedimensional printing. Biomaterials 26 (20), 4281-4289, http://dx.doi.org/10. 1016/j.biomaterials.2004.10.040.
Leong, K., Cheah, C., Chua, C., 2003. Solid freeform fabrication of three-dimensional scaffolds for engineering replacement tissues and organs. Biomaterials 24 (13), 2363-2378, http://dx.doi.org/10.1016/S0142-9612(03)00030-9.

Markicevic, B., Li, H., Zand, A.R., Navaz, H.K., 2012. Influence of the boundary conditions on capillary flow dynamics and liquid distribution in a porous medium. American Institute of Chemical Engineers Journal 58 (9.), http://dx.doi.org/10.1002/aic.

Vlasea, M., Shanjani, Y., Bothe, A., Kandel, R., Toyserkani, E., 2013. A combined additive manufacturing technique for realization of bio-ceramic structures with micro-scale channels. International Journal of Advanced Manufacturing Technology, http://dx.doi.org/10.1007/s00170-013-4839-7, 9 pp.

Vozzi, G., Previti, A., Rossi, D., Ahluwalia, A., 2002. Microsyringe-based deposition of two-dimensional and three-dimensional polymer scaffolds with a well-defined geometries for application to tissue engineering. Tissue Engineering 8 (6), 1089-1098.

Warnke, P.H., Seitz, H., Warnke, F., Becker, S.T., Sivananthan, S., Sherry, E., Liu, Q., et al., 2010. Ceramic scaffolds produced by computer-assisted 3D printing and sintering: characterization and biocompatibility investigations. Journal of Biomedical Materials Research. Part B, Applied Biomaterials 93 (1), 212-217, http://dx.doi.org/10.1002/jbm.b.31577.

Woesz, A., Rumpler, M., Stampf, J., Varga, F., Fratzl-zelman, N., Roschger, P., 2005. Towards bone replacement materials from calcium phosphates via rapid prototyping and ceramic gelcasting. Gene 25, 181-186, http://dx.doi.org/10.1016/j.msec.2005.01.014.

Yang, S., Leong, K.F., Du, Z., Chua, C.K., 2001. The design of scaffolds for use in tissue engineering. Part I. Traditional factors. Tissue Engineering 7 (6), 679-689, http://dx.doi.org/10.1089/107632701753337645.

Yang, Shoufeng, Leong, K.-F., Du, Z., Chua, C.-K., 2002. The design of scaffolds for use in tissue engineering. Part II. Rapid prototyping techniques. Tissue Engineering 8 (1), 1-11, http://dx.doi.org/10.1089/107632702753503009. 